Florida International University

FIU Digital Commons

$7-2-2020$

\title{
Structure Function Relationship in Hexacoordinate Heme Proteins: Mechanism of Globin X Interactions with Exogenous Ligands and Ligand Accessiblity in Cytoglobin and Neuroglobin
}

Ruipeng Lei

rlei001@fiu.edu

Follow this and additional works at: https://digitalcommons.fiu.edu/etd

Part of the Biochemistry Commons, and the Biophysics Commons

\section{Recommended Citation}

Lei, Ruipeng, "Structure Function Relationship in Hexacoordinate Heme Proteins: Mechanism of Globin X Interactions with Exogenous Ligands and Ligand Accessiblity in Cytoglobin and Neuroglobin" (2020). FIU Electronic Theses and Dissertations. 4470.

https://digitalcommons.fiu.edu/etd/4470

This work is brought to you for free and open access by the University Graduate School at FIU Digital Commons. It has been accepted for inclusion in FIU Electronic Theses and Dissertations by an authorized administrator of FIU Digital Commons. For more information, please contact dcc@fiu.edu. 


\section{FLORIDA INTERNATIONAL UNIVERSITY}

Miami, Florida

STRUCTURE-FUNCTION RELATIONSHIPS IN HEXACOORDINATE HEME PROTEINS: MECHANISM OF GLOBIN X INTERACTIONS WITH

EXOGENOUS LIGANDS AND LIGAND ACCESSIBILITY IN CYTOGLOBIN AND NEUROGLOBIN

A dissertation submitted in partial fulfillment of the

requirements for the degree of

DOCTOR OF PHILOSOPHY

in

BIOCHEMISTRY

by

Ruipeng Lei

2020 
To: Dean Michael R. Heithaus

College of Arts, Sciences and Education

This dissertation, written by Ruipeng Lei, and entitled Structure-Function Relationships in Hexacoordinate Heme Proteins: Mechanism of Globin X Interactions with Exogenous Ligands and Ligand Accessibility in Cytoglobin and Neuroglobin, having been approved in respect to style and intellectual content, is referred to you for your judgement.

We have read this dissertation and recommend that it be approved.

Yuk-Ching Tse-Dinh

$\begin{array}{r}\hline \text { Irina Agoulnik } \\ \hline \text { Xiaotang Wang } \\ \hline \text { Jaroslava Miksovska, Major Professor }\end{array}$

Date of Defense: July 2, 2020

The dissertation of Ruipeng Lei is approved.

Dean Michael R. Heithaus

College of Arts, Sciences and Education

Andrés G. Gil

Vice President for Research and Economic Development and Dean of the University Graduate School 
(C) Copyright 2020 by Ruipeng Lei

All rights reserved. 


\section{DEDICATION}

I dedicate this work to my parents. Without their patience, understanding, support, and selfless love, the completion of this work would not have been possible. 


\section{ACKNOWLEGMENTS}

I would like to express my gratitude to my major Professor, Dr. Jaroslava Miksovska, for giving me the opportunity to join her lab and to work on this project, for providing me with knowledge and techniques to pursue my project, and for encouraging independent study and professional development. I greatly appreciate all her mentoring and encouragement to let me present my work on several national and international conferences which I believe has greatly expand my horizon in the field of scientific research.

I would like to thank my committee members, Dr. Yuk-Ching Tse-Dinh, Dr. Irina Agoulnik, and Dr. Wang, for taking time to provide me with insightful suggestions to improve my research as well as generous support to continue and finish my $\mathrm{Ph} . \mathrm{D}$. career steadfast.

I wish to thank my former and current lab mates-Dr. Antonija Tanger, Dr. Khoa Pham, Dr. David Butcher, Dr. Walter Gonzalez, Maria Santiago, and Setareh Sakhdari, for their dedications, support and great times in the lab. In particular, I would like to thank all the undergraduate students who work with me, Maria Jose Santiago, Manuel Picon and Isa Sabir, for their contributions to both published and unpublished results.

I especially appreciate my family for all their encouragement and support during all these years. I am indebted to my parents for all their unconditional love, dedication and sacrifice to give me the best education to their most, which makes me fearless and energetic in pursuing the career I like. 
I will also give special thanks to my wife Li Mo. Without her help, love and encouragement to keep me moving forward, I can't make it so far.

I also would like to thank the Department of Chemistry and Biochemistry at FIU for accepting me into their graduate program, and for their financial support as Teaching Assistant. In addition, I would like to acknowledge the financial support provided by FIU graduate school for the Dissertation Year Fellowship, which greatly helped me preparing for my graduation. 


\author{
ABSTRACT OF THE DISSERTATION \\ STRUCTURE-FUNCTION RELATIONSHIPS IN HEXACOORDINATE \\ HEME PROTEINS: MECHANISM OF GLOBIN X INTERACTIONS WITH \\ EXOGENOUS LIGANDS AND LIGAND ACCESSIBILITY IN CYTOGLOBIN
}

AND NEUROGLOBIN

by

Ruipeng Lei

Florida International University, 2020

Miami, Florida

Professor Jaroslava Miksovska, Major Professor

Cytoglobin (Cygb), neuroglobin ( $\mathrm{Ngb})$, and globin $\mathrm{X}(\mathrm{GbX})$ belongs to recently discovered members of the vertebrate globin family, they carry a heme prosthetic group that can reversibly bind exogenous ligands such as $\mathrm{CO}, \mathrm{NO}$ and $\mathrm{O}_{2}$. Although the physiological functions of Cygb, $\mathrm{Ngb}$ and $\mathrm{GbX}$ are still under debate, several possible physiological functions for these proteins were proposed. Cytoglobin was reported to participate in lipid-based signaling and to stabilize the tumor suppressor p53 upon DNA damage, which imply its anti-cancer role. Neuroglobin was shown to interact with $\alpha$ subunit of the heterotrimeric $\mathrm{G}$ protein as well as cytochrome $\mathrm{c}$ which indicate a role in cell apoptosis. Both proteins were also proposed to participate in NO metabolism. Compared to the well-known vertebrate globin, hemoglobin and myoglobin, the new members have several distinct structural characteristics. First, unlike $\mathrm{Hb}$ and $\mathrm{Mb}$, the 
distal histidine coordinates with the heme iron at the sixth axial position in Cygb, Ngb and $\mathrm{GbX}$, forming a hexa-coordinated heme iron and thus regulating kinetics and equilibrium constants for exogenous ligand binding to heme. Second, an oxidation/reduction of an intramolecular disulfide bridge which is found in all three hexa-coordinated globins, also modulates affinity for diatomic ligands such as $\mathrm{O}_{2}$ and CO. Additionally, both Cygb and GbX are found to have extended N- and C- terminals with unclear function, although the $\mathrm{N}$-terminal in $\mathrm{GbX}$ proposed to be involved in the protein binding to the membrane.

The work presented in this dissertation focuses on investigation of the role of internal ligand (distal histidine) and disulfide bridge on structure-function relationships in $\mathrm{GbX}$, in terms of regulating affinity and kinetics for small diatomic ligands. Indeed, we shown a very weak ligand binding to heme iron in $\mathrm{GbX}$, suggesting its district role among heaxa-coordiante vertebrate globins. In addition, the study of conformation dynamics that affect the heme cavity accessibility of Cygb and Ngb by incorporate heme fluorescent analogy ZnPPIX into the protein is also performed. These data shown a high conformational heterogeneity of the distal pocket in hexa-coordiante globins as well as increased accessibility of the heme pocket in Ngb. 


\section{TABLE OF CONTENTS}

CHAPTER

PAGE

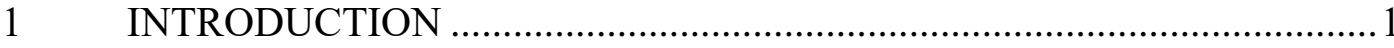

1.1 Metallo-porphyrin and Heme proteins ....................................................... 1

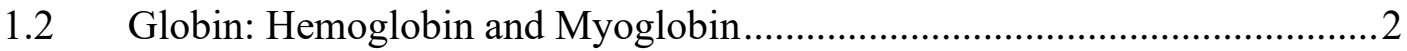

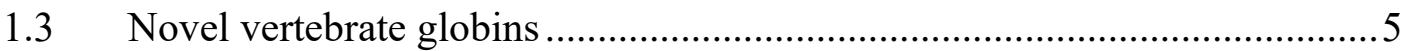

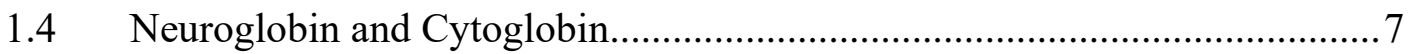

1.5 GbX: a membrane-bound vertebrate globin ............................................ 11

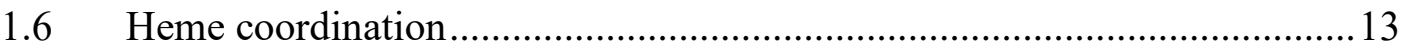

1.7 Disulfide bridge modulate protein-ligand interaction and protein stability. 14

1.8 Distal histidine: gate of the distal pocket................................................ 16

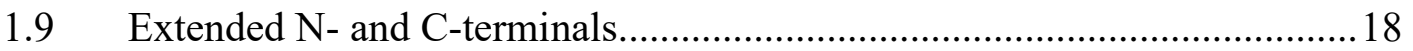

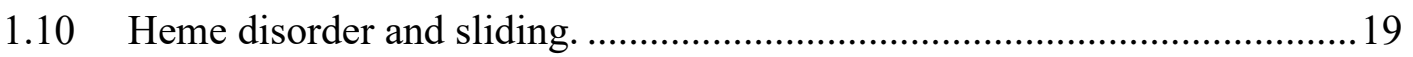

1.11. Globin interactions with exogenous ligands .......................................... 21

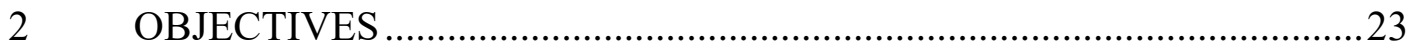

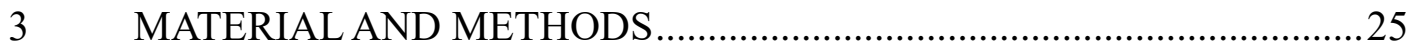

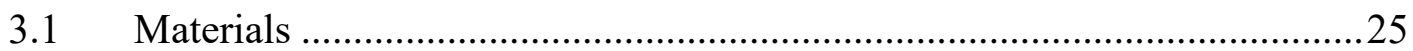

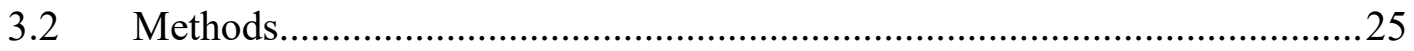

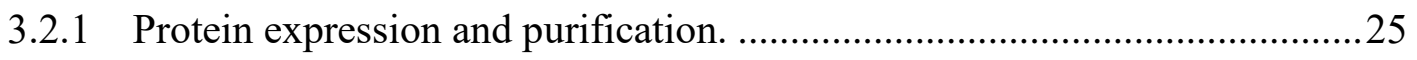

3.2.1.1 Neuroglobin and Cytoglobin isolation and purification. .......................25

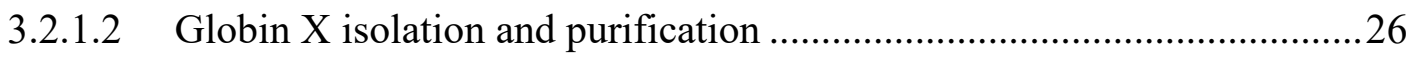

3.2.2 Sodium dodecyl sulfate electrophoresis...................................................2

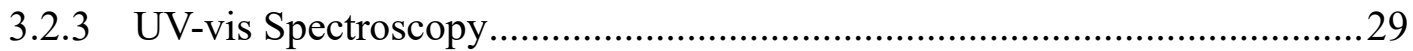

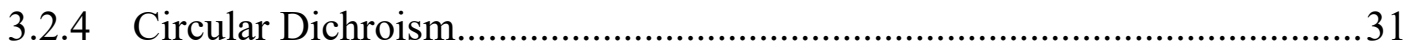

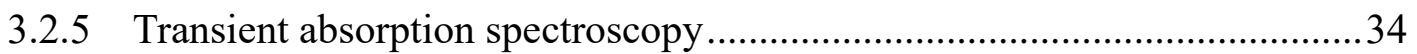

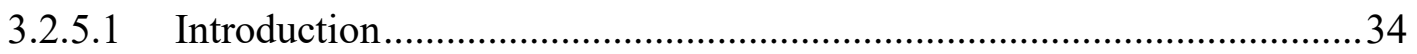

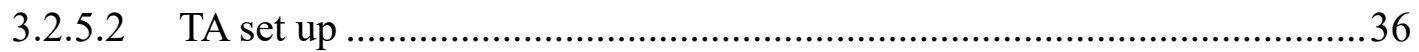

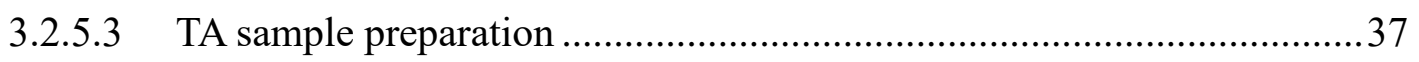

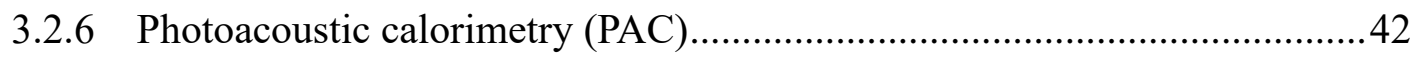

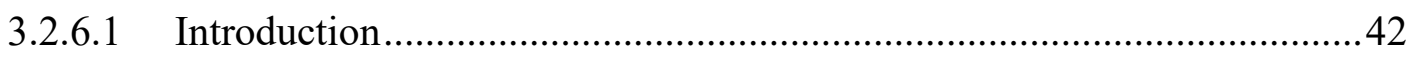

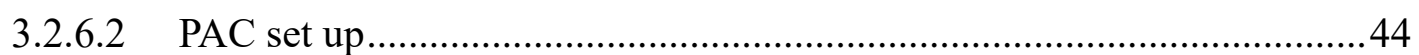

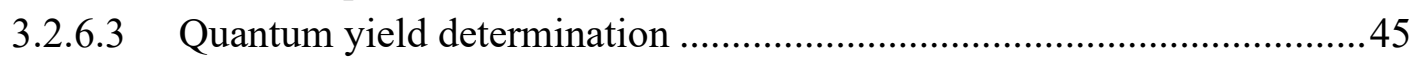

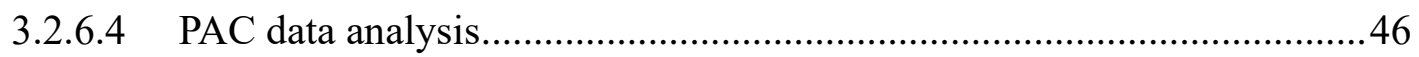

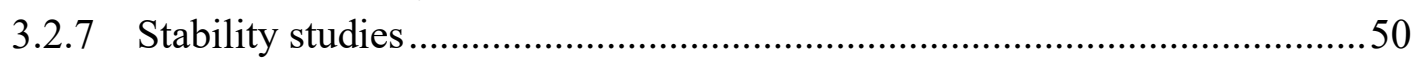

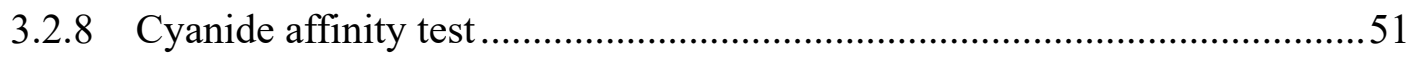

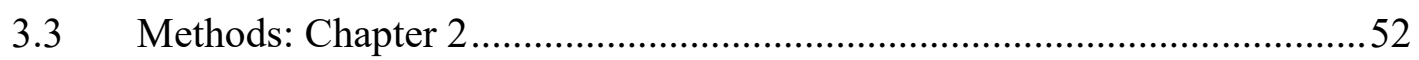

3.3.1 Preparation of ZnPPIX reconstituted hexacoordinate globin .....................55

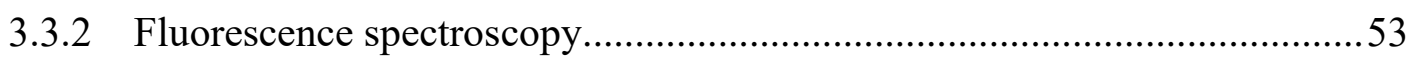


3.3.2.1 Steady-state fluorescence spectroscopy ................................................54

3.3.2.2 Steady-state frequency-domain fluorescence lifetime ............................55

3.3.2.3 Time-resolved fluorescence lifetime measurement ...............................57

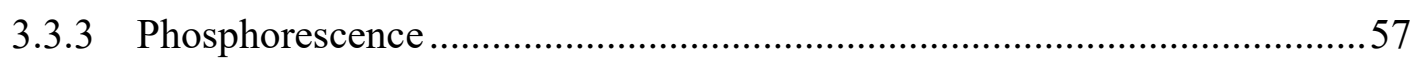

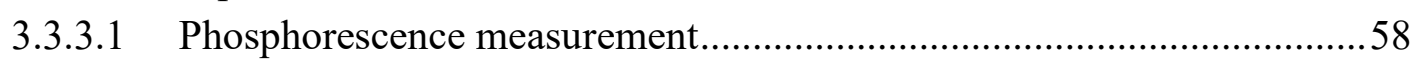

4 IMPACT OF THE DISULFID BRIDGE AND DISTAL HISTIDINE ON

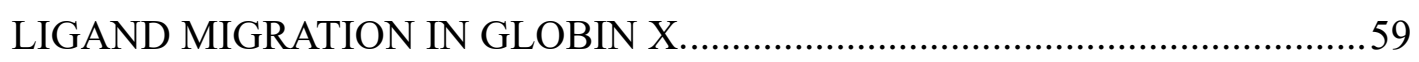

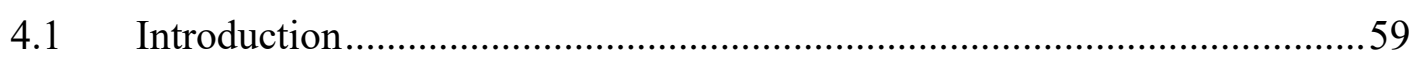

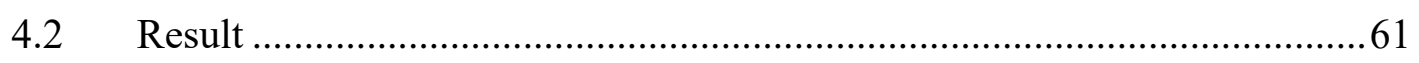

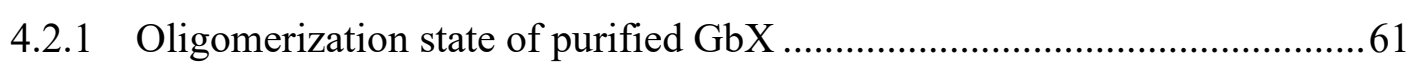

4.2.2 Steady-state UV-vis spectra and far-UV CD spectra ................................63

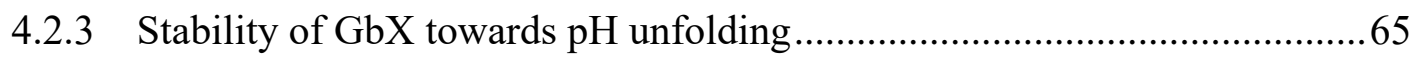

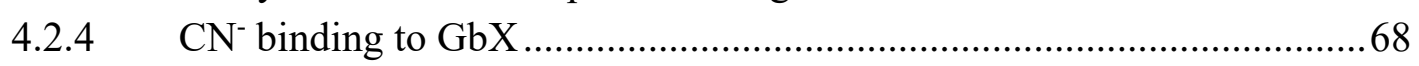

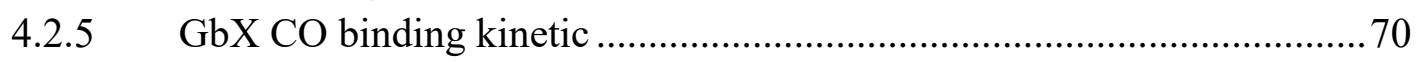

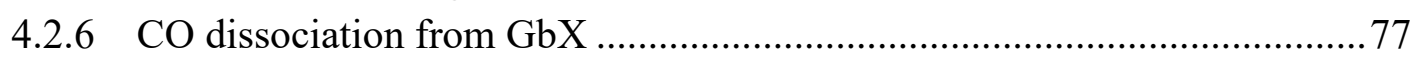

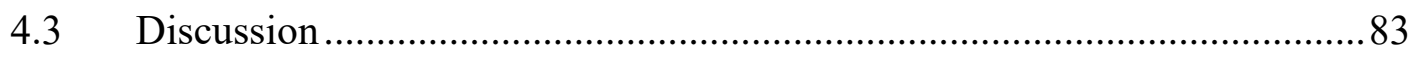

5 CHARACTERIZATION OF THE CONFORMATION, REGULATION, ORIENTATION, AND HEME ACCESIBILITY IN HEXACOORDINATE GLOBINS BY USING FLUORESCENT HEME ANALOG. ..............................86

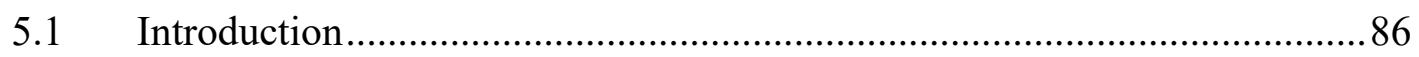

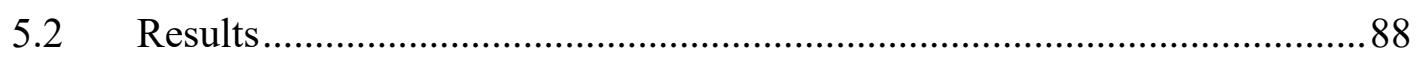

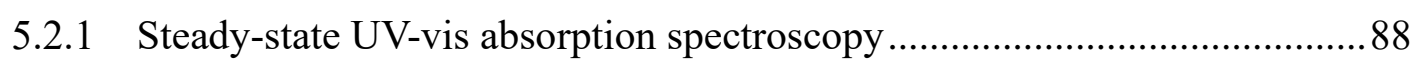

5.2.2 Steady-state fluorescence emission spectra ............................................ 90

5.2.3 Fluorescence and phosphorescence lifetime ........................................... 92

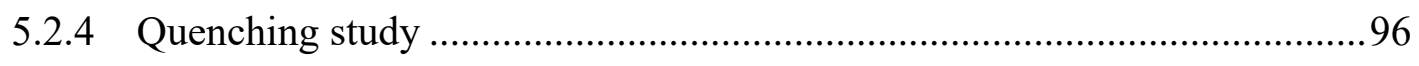

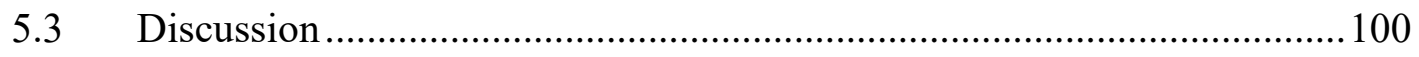

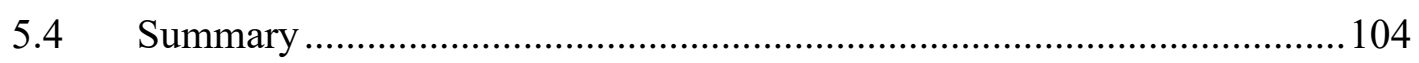

6 THE $\mathrm{O}_{2}$ BINGING KINETIC AND IMPACT ON HEME CAVITY

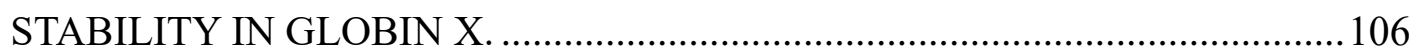

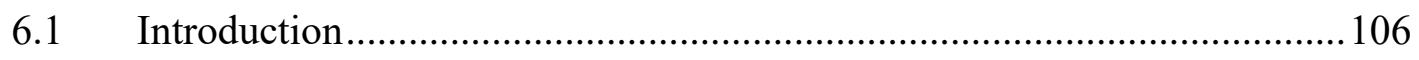

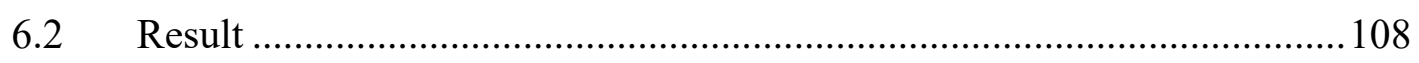

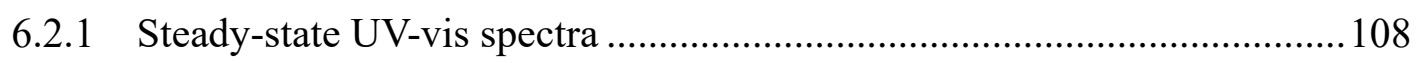

6.2.2 Stability of $\mathrm{O}_{2}$ bound $\mathrm{GbX}$ towards $\mathrm{pH}$ unfolding.................................. 109

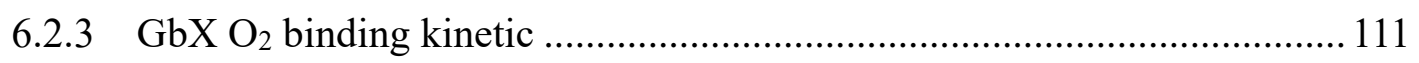

6.2.4 Photoacoustic calorimetry result of $\mathrm{O}_{2}$ dissociation ................................ 113

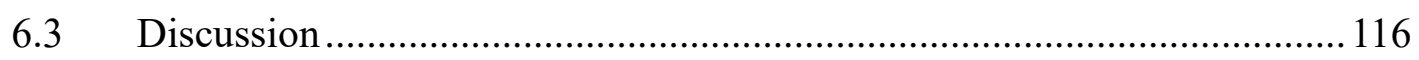




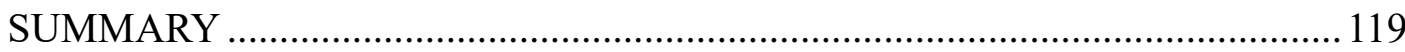

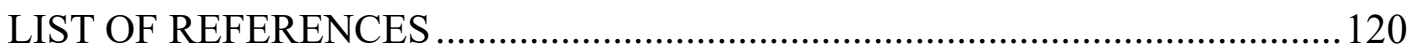

VITA 


\section{LIST OF TABLES}

TABLE

PAGE

Table 3.1.Ingredient of 1L 10X SDS running buffer for electrophoresis. .28

Table 3.2. Ingredient of loading buffer for electrophoresis. .28

Table 3.3. Ingredient of Coomassie blue staining buffer.

Table 3.4. Ingredient of de-staining buffer. .29

Table 3.5. The electromagnetic spectrum (Worsfold \& Zagatto, 2005) .30

Table 3.6. The visible spectrum (Worsfold \& Zagatto, 2005). 31

Table 4.1 UV-vis absorption spectra wavelength of Soret and $\alpha / \beta$ band of each GbX variants.

Table 4.2. Parameters of the $\mathrm{pH}$-induced GbX variants unfolding (* (Picotti et al., 2009)).

Table 4.4. Rate constants for $\mathrm{CO}$ binding to $\mathrm{GbX}$ variants obtained by fitting the experimental data by exponential decay model and MEM analysis at $20^{\circ} \mathrm{C}$. (*(Belogortseva et al., 2007), (Butcher et al., 2017), ¥(Astudillo et al., 2013) )

Table 4.5. Activation energy, log pre-exponential factor, activation enthalpy, and activation entropy of temperature dependent $\mathrm{CO}$ rebinding to $\mathrm{GbX}$ variants. (*(Butcher et al., 2017), †(Mikšovská et al., 2003))

Table 4.6. Rate and equilibrium constants for $\mathrm{CO}$ binding to $\mathrm{GbX}$ variants. 
Table 4.7. Reaction $(\Delta \mathrm{H}$ and $\Delta \mathrm{V})$ and activation $\left(\Delta \mathrm{H}^{\#}\right.$ and $\left.\Delta \mathrm{V}^{\#}\right)$ parameters associated with the photo-dissociation of $\mathrm{Fe}-\mathrm{CO}$ bond and subsequent ligand escape from the protein matrix in GbX variants. (*(Astudillo et al., 2013))

Table 4.8. Total volume and enthalpy changes associated with the CO dissociation from $\mathrm{GbX}$ variants as well as Cygb, $\mathrm{Ngb}, \mathrm{Mb}$, and $\mathrm{Hb}$. (*(Astudillo et al., 2013), †(Peters et al., 1992)).

Table 5.1. Summary of UV-vis absorption maxima for ZnPPIX reconstituted hhMb, Cygb and Ngb variants. Specifically, CygbR84L shown a shoulder rather than a peak at $\sim 595 \mathrm{~nm}$.

Table 5.2. Summary of fluorescence emission maxima for ZnPPIX reconstituted hhMb, Cygb and Ngb variants.

Table 5.3. Summary of fluorescence and phosphorescence parameters of ZnPPIXreconstituted hexacoordinate globins.

Table 5.4. Summary of Stern-Volmer constant of ZnPPIX-reconstituted hexacoordinate globins.

Table 5.5. Summary of quenching rate constant of ZnPPIX-reconstituted globins... 100

Table 6.1. Absorption spectra wavelength of Soret and Q-band of GbX WT and C64A mutant summarized in the table. 108

Table 6.2. Parameters of the acid-induced oxygen bound GbX variants unfolding. . 111

Table 6.3. Rate constants for $\mathrm{O}_{2}$ binding to $\mathrm{GbX}$ variants obtained using both 
Table 6.4. Activation energy, log pre-exponential factor, activation enthalpy, and activation entropy of temperature dependent $\mathrm{O}_{2}$ rebinding to $\mathrm{GbX}$ variants.

Table 6.5. Thermodynamic parameters associated with $\mathrm{O}_{2}$ photo-dissociation from GbXWT and GbXC65A. (*(Astudillo, 2014)) 


\section{LIST OF FIGURES}

FIGURE

PAGE

Figure 1.1. Examples of metallo-porphyrins. (A) iron protoporphyrin IX. (B) zinc protoporphyrin IX. (C) magnesium protoporphyrin IX. (D) copper protoporphyrin IX.

Figure 1.2 Ribbon representation of the three-dimensional structure of horse heart $\mathrm{Hb}$ with $\alpha$ subunits in yellow and $\beta$ subunits in pink (left, PDB entry: 2HHB) and sperm whale myoglobin (right PDB entry: $1 \mathrm{~A} 6 \mathrm{~N}$,). The, heme group is shown in red.

Figure 1.3. Simplified phylogenetic relationships in vertebrate globins by using uniport database along with their coordination type ( $\mathrm{GbY}$ is yet determined).

Figure 1.4. Ribbon representation of human neuroglobin (left, PDB: 4MPM chain A) and cytoglobin (right, remodeled by PDB: 2DC3). The proximal and distal histidine is shown in sticks, the heme is shown in red, two cysteine residues are in orange. The cytoglobin extended $\mathrm{N}$ terminal is shown in yellow and $\mathrm{C}$ terminal is shown in gray.

Figure 1.5. GbX sequence alignment with vertebrate globins and predicted secondary structures. Highly conserved amino acid residues are labeled with blue shadows.

Figure 1.6. Ribbon representation of the heme binding site in vertebrate $\mathrm{Mb}$ (left, PDB entry $1 \mathrm{~A} 6 \mathrm{~N}$ ), Ngb (right, PDB entry 4MPM) demonstrating pentacoordination and hexa-coordination respectively, of the heme iron. The distal (magenta) and proximal (green) histidine residues are shown as sticks.

Figure 1.7. The heme orientations in $\mathrm{Mb}(\mathrm{A})$ and rotated $180^{\circ}$ about the $\alpha-\gamma$-meso axis (B). The heme substituents are labeled $\mathrm{M}$ (methyl), $\mathrm{V}$ (vinyl), and $\mathrm{P}$ 
Figure 1.8. Heme sliding upon $\mathrm{CO}$ binding. Ferric murine Ngb (in green, PDB: 5EET), CO bound murine Ngb (in cyan, PDB: 1W92). Distal and proximal histidine, heme group as well as Phe-106 are also shown as sticks

Figure 3.6. Sample electronic CD spectra of protein in $\alpha$-helix, a $\beta$-sheet and a random coil. Simulated by PDBMD2CD.com with data from protein data bank.

Figure 3.7. In transient absorption spectroscopy, time profiles of absorption changes that are associated with ligand binding/dissociation from proteins are measured. (A) Absorption spectrum of deoxy- and $\mathrm{CO}-\mathrm{GbX}$ in the UV and visible (inset) region. (B) Simplified reaction diagram of $\mathrm{CO}$ photodissociation and rebinding to swMb. (C) Time resolved absorption trance for $\mathrm{CO}$ recombination to $\mathrm{GbX}$ on microsecond to millisecond timescale.

Figure 3.8. Top-view schematic of the transient absorption apparatus. TA components: the sample (red), temperature-controlled cuvette holder (TC), mirrors, pump beam (Nd:YAG532), beam blocker (B), lenses, probe beam (Xe lamp), monochromator (MC), photodiode (D) and Digitizer. Dashed line indicates pulsed light.

Figure 3.9. Contour plots of the statistic parameter $\chi^{2}$ and the entropy $S$ for a two dimensional $f(\lambda)$. The maximum entropy solution equivalent to the point where the gradient of $\chi^{2}$ is parallel to the gradient of $\mathrm{S}$, where $\chi^{2}$ is close to 1 . Modified from Steinbach et al. (Steinbach et al., 1992)..

Figure 3.10. An energy diagram for $\mathrm{CO}$ binding to $\mathrm{Mb}$. .42

Figure 3.11. Schematic diagram of a home-build PAC instrument setup (top view). .43

Figure 3.12. Schematic diagram of wave propagation from the sample to the 
Figure 3.13. Illustrative PAC acoustic traces for sample and reference compound. ...47

Figure 3.14. Illustrative PAC acoustic traces for the sample acoustic trace is shifted in phase compare to the reference trace.

Figure 3.15. Simplified Jablonski diagram repersent electronic transitions between different electronic states. A as absorption, IC as internal conversion, ISC as intersystem crossing, $\mathrm{F}$ as fluorescence, $\mathrm{NR}$ as nonradiative decay, $\mathrm{P}$ as phosphorescence, $\mathrm{S}$ as singlet state, $\mathrm{T}$ as triplet state.

Figure 3.16. The modulation of emission (red) is decreased by modulation of excitation light intensity (black) and result in phase shift. The dash line indicated average intensity for both waves. The modulation ratio $(\mathrm{m})$ is determined by the amplitude of the average intensity (a, A) and the offset from the average intensity $(b, B)$ of emission and excitation.

Figure 3.17. Phase delay $\left(\phi_{\omega}\right)$ and modulation ratio $(\mathrm{m})$ versus modulation frequency $(\omega)$ for POPOP reference compound.

Figure 4.1. SDS-PAGE gel for purified GbX variants in the presence/absence of $\beta$ me. B, SDS-PAGE gel for GbXWT fractions after separation of monomer and dimer using size exclusion chromatography.

Figure 4.2. Absorbance spectra of the oxidized (met), reduced (ferrous deoxy), and $\mathrm{CO}$ bound forms of $\mathrm{GbX}$ variants.

Figure 4.3. $\mathrm{CD}$ spectra in the far-UV region of $\mathrm{GbX}$ variants. All samples are aligned at $\mathrm{Abs}_{280} \mathrm{~nm}$.

Figure 4.4. Acid-induced unfolding of GbXWT UV-vis absorption spectra. 
Measurements were performed in $5 \mathrm{mM}$ phosphate-citrate buffer and $100 \mathrm{mM}$ $\mathrm{NaCl}$, under equilibrium conditions

Figure 4.5. Acid-induced GbX variants unfolding. The solid line corresponds to the experimental data using Eq. 3.36. Measurements were performed in $5 \mathrm{mM}$ phosphate-citrate buffer and $100 \mathrm{mM} \mathrm{NaCl}$, under equilibrium conditions

Figure 4.6. A, absorption difference spectra at various cyanide concentrations, relative to the ferric $\mathrm{GbX}$ form (without cyanide), the spectra were measured at room temperature in $50 \mathrm{mM}$ Tris $\mathrm{HCl}$ at $\mathrm{pH} \mathrm{7.} \mathrm{B,} \mathrm{cyanide} \mathrm{binding} \mathrm{to} \mathrm{GbX}$ variants, fraction bond was calculated by absorption at $410 \mathrm{~nm}$ as a function of cyanide concentration, only cysteine mutant shows possible two binding sites.

Table 4.3. Parameters of the $\mathrm{CN}^{-}$binding to $\mathrm{GbX}$ variants (†(Tsujino et al., 2014), $\neq($ Dou et al., 1996))

Figure 4.7. A, transient absorption traces for $\mathrm{CO}$ rebinding to $\mathrm{GbX}$ variants. $\mathrm{B}$, Lifetime distribution associated with the $\mathrm{CO}$ rebinding to $\mathrm{GbX}$ variant determined by MEM approach. $\mathrm{C}$, Arrhenius plot of temperature dependent $\mathrm{CO}$ rebinding to $\mathrm{GbX}$ variants, color represent different rate constant (black k1, red k2, blue k3, and purple k4) and symbol represent different GbX variants (square WT, round C65A, and triangle $\mathrm{H} 90 \mathrm{~V})$. D, Eyring plot of temperature dependent $\mathrm{CO}$ rebinding to $\mathrm{GbX}$ variants.

Figure 4.8. Logarithmic plots of the rate constants for $\mathrm{CO}$ rebinding to GbXWT (A), GbXC65A (B), and $\mathrm{GbXH90V} \mathrm{(C)} \mathrm{as} \mathrm{a} \mathrm{function} \mathrm{of} \mathrm{CO}$ concentration, experiment was performed by $20 \mu \mathrm{M}$ protein in $50 \mathrm{mM}$ TrisHCl, $\mathrm{pH} 7$, at $25^{\circ} \mathrm{C} \ldots \ldots . . .74$

Figure 4.9. $\mathrm{CO}$ quantum yield for bimolecular dissociation from $\mathrm{GbX}$ variants as a function of temperature.

Figure 4.10. Overlay of normalized acoustic traces of $\mathrm{CO}$ bound $\mathrm{GbX}$ variants with 
reference...

Figure 4.11. Arrhenius (left) and Eyring (right) plot of slow phase for CO photodissociation from GbXWT and C65A mutant.

Figure 4.12. Plot of $\phi_{i} E_{h v}$ versus $C_{p} \rho / \beta$ for the prompt phase (left) and the slow phase (right) for $\mathrm{CO}$ photo-dissociation from $\mathrm{GbX}$ variants.

Figure 5.1. Normalized steady-state absorption spectra of ZnPPIX-hhMb and ZnPPIX-Cygb variants (A) and ZnPPIX-Ngb variants (B) in $50 \mathrm{mM}$ TrisHCl pH 7.0 .

Figure 5.1. Normalized steady-state fluorescence emission of ZnPPIX-Cygb variants (A) and ZnPPIX-Ngb variants (B), using $\lambda_{\mathrm{exc}}=421 \mathrm{~nm}$.

Figure 5.3. Time-resolved fluorescence in the frequency domain data determined for ZnPPIX-Cygb variants (A) and ZnPPIX-Ngb variants (B).

Figure 5.4. Time-resolved fluorescence in the frequency domain data determined for ZnPPIX-Cygb variants (A) and ZnPPIX-Ngb variants (B).

Figure 5.5. Phosphorescence decay determined for reconstituted globins. Phosphorescence decay was monitored at $447 \mathrm{~nm}$.

Figure 5.6. Example of UV-vis absorption spectra of ZnPPIX reconstituted protein with increasing concentration of methyl viologen. A decrease of Soret band absorbance is observed.

Figure 5.7. Example of fluorescence emission spectra of ZnPPIX reconstituted 
Figure 5.8. The Stern-Volmer plot for the quenching of the steady-state fluorescence of $\mathrm{ZnPPIX}$ reconstituted globins by methyl viologen.

Figure 5.9. Triplet state lifetime traces of ZnPPIX-hhMb obtained by transient absorbance at varying concentration of oxygen. All traces are fitted by single exponential decay.

Figure 5.10. The Stern-Volmer plot for the quenching of the triplet-state phosphorescence of ZnPPIX reconstituted hhM by oxygen.

Figure 6.1. Absorption spectra of the oxidized (met), reduced (ferrous deoxy), and $\mathrm{O}_{2}$ bound forms of GbX WT and C65A mutant. Measured in $50 \mathrm{mM}$ TrisHCl pH 7.0

Figure 6.2. UV-vis absorption spectra of GBXWT in the oxygen bound form as function of $\mathrm{pH}$. Measurements were performed in $5 \mathrm{mM}$ phosphate-citrate buffer and $100 \mathrm{mM} \mathrm{NaCl}$, under equilibrium conditions.

Figure 6.3. Fraction unfolding of oxygen bound $\mathrm{GbX}$ WT and C65A mutant plot as a function of $\mathrm{pH}$.

Figure 6.4. A, transient absorption traces for $\mathrm{O}_{2}$ rebinding to $\mathrm{GbX}$ variants. $\mathrm{B}$, Lifetime distribution associated with the $\mathrm{O}_{2}$ rebinding to $\mathrm{GbX}$ variant determined by MEM approach. $\mathrm{C}$, Arrhenius plot of temperature dependent $\mathrm{O}_{2}$ rebinding to $\mathrm{GbX}$ variants. D, Eyring plot of temperature dependent $\mathrm{O}_{2}$ rebinding to $\mathrm{GbX}$ variants.

Figure 6.5 Quantum yiled of $\mathrm{O}_{2}$ from GbXWT and C65A plot as a function of 
temperature.

Figure 6.6 Photoacoustic traces for oxygen dissociation from GbXWT (left) and GbXC65A (right) together with the trace for the reference compound 4SP. Conditions: $20 \mu \mathrm{M}$ protein in $50 \mathrm{mM}$ Tris buffer ( $\mathrm{pH}$ 7.0). The absorbance of the reference compound is aligned with the absorbance of the sample at $532 \mathrm{~nm}$.

Figure 6.7. Plot of $\varphi$ Ehv as a function of $\mathrm{Cp} \rho / \beta$ for $\mathrm{O}_{2}$ photo-release from $\mathrm{GbXWT}$ (black), and GbXC65A (red). The associated volume and enthalpy changes were obtained from the slope and intercept of the linear fits, respectively. 


\section{LIST OF ABBREVIATIONS AND ACRONYMS}

ABBREVIATION

FULL NAME

AA

Amino acid

$\mathrm{CO}$

Carbon monoxide

CygbWT

Cytoglobin wild-type

DTT

Dithiothreitol

FePPIX

Iron protoporphyrin IX

GbXWT

Globin X wild-type

$\mathrm{Hb}$

Hemoglobin

$\mathrm{hhMb}$

Horse heart myoglobin

$\Delta \mathrm{H}$

Enthalpy change

$\Delta \mathrm{H}^{\ddagger}$

Activation enthalpy change

$\mathrm{K}_{\mathrm{d}}$

Dissociation constant

$\mathrm{Mb}$

Myoglobin

$\mathrm{NaCN}$

Sodium syanide

NgbWT

Neuroglobin wild-type

$\mathrm{NO}$

Nitric oxide

$\mathrm{O}_{2}$

Oxygen

PAC

Photoacoustic calorimetry

$\Delta \mathrm{S}$

Entropy change 


\section{INTRODUCTION}

\subsection{Metallo-porphyrin and Heme proteins}

Metallo-porphyrins represent a diverse class of coordination complexes with a cyclic structure. They are often found as prosthetic groups in various proteins as they can catalyze a remarkable number of catalytic reactions and carrying additional functions such as an electron transport or ligand binding (figure 1.1)(Chandra et al., 2000; Larsen \& Mikšovská, 2007). Among all the metallo-porphyrins, the ironprotoporphyrin IX (Heme b) is found in numerous proteins with distinct functions (Poulos, 2014). Heme b is a porphyrin derivative that is composed of four pyrrole rings liked by methine bridges with an iron coordinated to the nitrogen atom of the four pyrrole rings. The porphyrin ring has several substituents: four methyl groups, two propionate groups, and two vinyl substitutional groups. In heme proteins, heme b is incorporated into a protein matrix and stabilized through covalent, in case of cytochrome c, or noncovalent hydrophobic interactions between the prosthetic group and the amino acid residues. In addition, a coordination bond(s) between the heme iron and heteroatoms from amino acid sidechains contribute to the heme binding to the apoprotein (Voet \& Voet, 2010).

Heme proteins are extraordinary versatile and perform various functions such as catalysis (catalases), peroxidases (horseradish peroxidase), respiratory functions like oxygen storage and transport (myoglobin and hemoglobin), electron transfer (cytochromes), and oxygen sensors (HemAT) (Larsen \& Mikšovská, 2007). Although 
the heme group is in the active center of the protein, its properties including catalytic functions are fine-tuned by the amino acid residues surrounding heme and/or by residues participating in a coordination bond with the heme iron (histidine, methionine, or tyrosine) or additional structural properties such as hydrogen bonds and salt bridges between the amino acid side chains in the heme pocket and the propionate groups of the heme impact the stability of the heme group as well as interactions with diatomic ligands. (Anderson \& Chapman, 2005; Larsen \& Mikšovská, 2007).

$\mathbf{A}$

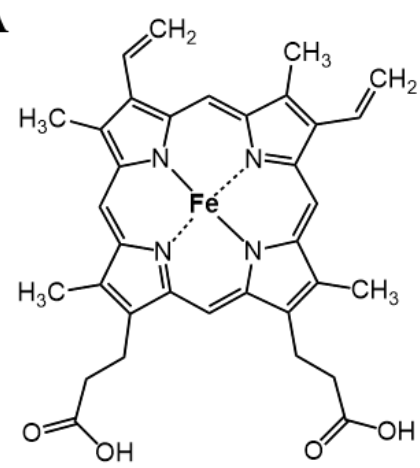

C

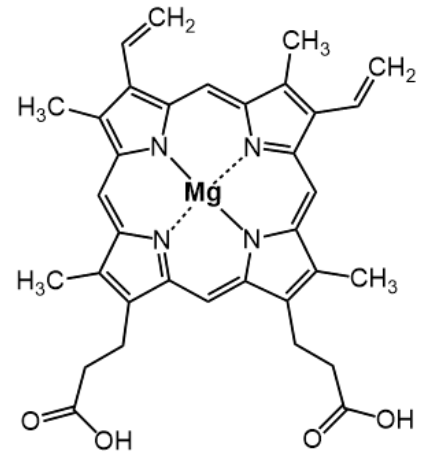

B

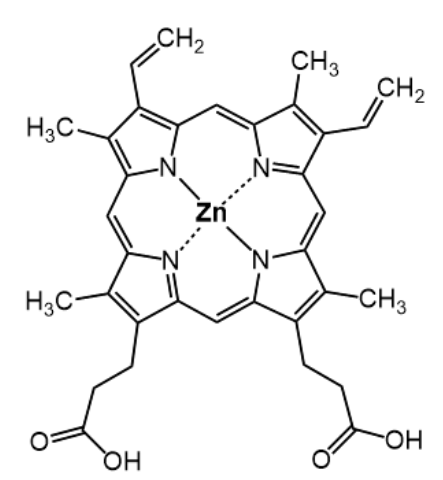

D

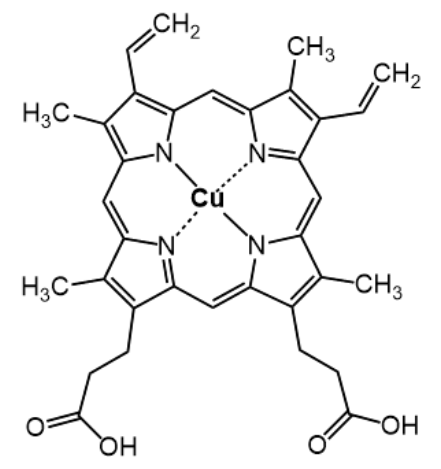

Figure 1.1. Examples of metallo-porphyrins. (A) iron protoporphyrin IX. (B) zinc protoporphyrin IX. (C) magnesium protoporphyrin IX. (D) copper protoporphyrin IX.

\subsection{Globin: Hemoglobin and Myoglobin}

Globins are a sub superfamily of the heme-proteins. They represent one of the most extensively studied group of proteins and serve as model for cooperative binding 
and allosteric regulation as they reversibly bind small diatomic ligands such as $\mathrm{CO}$, $\mathrm{NO}$, and $\mathrm{O}_{2}$ (Burmester \& Hankeln, 2014). Most globins consist of eight $\alpha$-helices (named A to $\mathrm{H}$ ) with a typical 3-over-3 $\alpha$-helical sandwich structure with the heme prosthetic group inside named globin fold. Globins was discovered in a variety of taxa, including bacteria, plants and animals. Among all the globins discovered to date, hemoglobin $(\mathrm{Hb})$ and myoglobin $(\mathrm{Mb})$ are the two globins that have been extensively studied and characterized (figure 1.2). Hemoglobin was discovered in 1840 by Friedrich Ludwig Hunefeld, and it is identified in different species — nearly all vertebrates, some invertebrates as well as some plants and fungi (Sheftel et al., 2011). In general, hemoglobin is a heterotetramer with a molecular weight of $65 \mathrm{kDa}$ consisting of two different subunits $\alpha$ and $\beta$. The red color of the vertebrate blood is the result of the high concentration of $\mathrm{Hb}$ in the erythrocytes. The main function of hemoglobin is to transport $\mathrm{O}_{2}$ from the respiratory surfaces (such as lungs, gills or skin) to the inner organs through the circulatory system (Voet \& Voet, 2010). The reason why large or complex organisms need hemoglobin is because the diffusion rate of $\mathrm{O}_{2}$ through tissue thicker than $\sim 1 \mathrm{~mm}$ is too slow and the solubility of $\mathrm{O}_{2}$ in blood plasma is too low to carry sufficient amounts of $\mathrm{O}_{2}$ to support large life forms (Voet \& Voet, 2010). Fortunately, with the help of hemoglobin, blood can carry $\mathrm{O}_{2}$ at a concentration up to $0.01 \mathrm{M}$ which is about the same concentration of $\mathrm{O}_{2}$ in the air (Voet \& Voet, 2010). Other than $\mathrm{O}_{2}$ transportation, nitric oxide (NO) metabolism is another important aspect of vertebrate $\mathrm{Hb}$ function. In the oxygen bond form, hemoglobin can scavenge excess 
of toxic NO and convert it to nitrate. In the deoxy form, hemoglobin can produce NO from nitrite reservoir which can initiate blood vessel dilatation during hypoxia (Burmester \& Hankeln, 2014). Although the hemoglobin is functionally similar between different species, hemoglobins isolated from different species are structurally distinct and have different ligand binding cooperativity and allosteric regulation. Comparison of sequence/structure/function relationship among hemoglobins provides valuable information on how protein evolved to assist animal adaptation to physiologically challenging habitats such as high altitude or cold weather (Burmester \& Hankeln, 2014; Storz \& Moriyama, 2008).
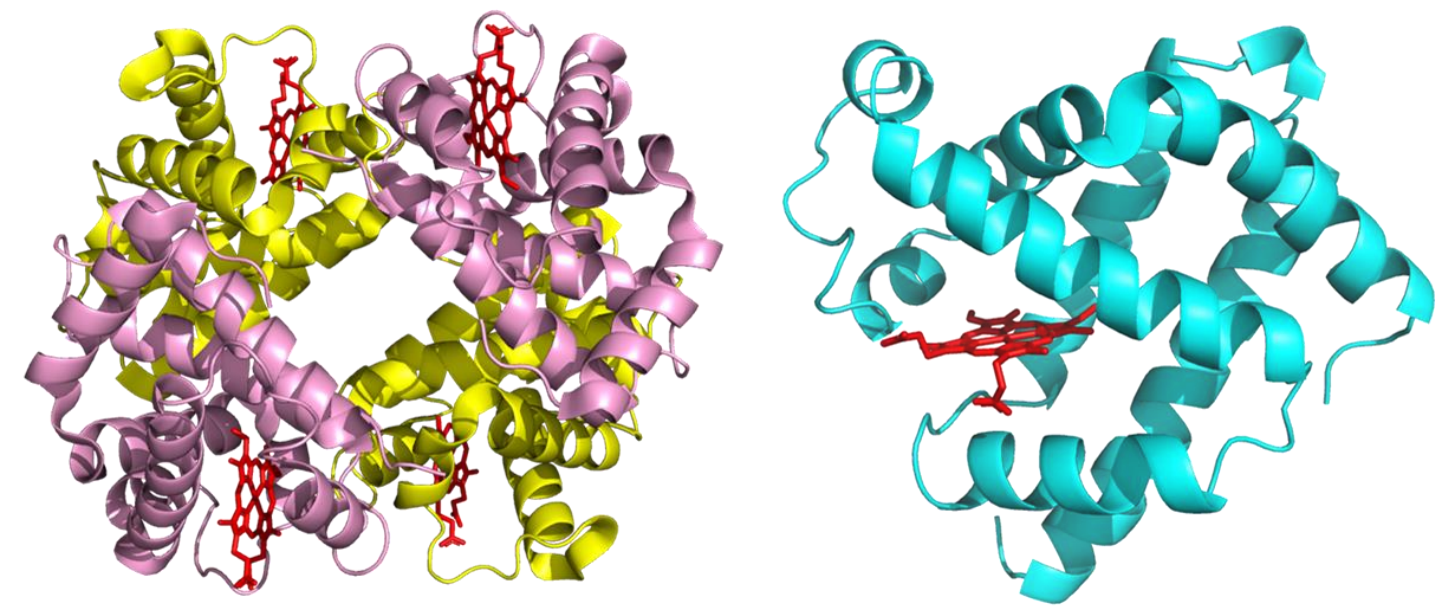

Figure 1.2 Ribbon representation of the three-dimensional structure of horse heart $\mathrm{Hb}$ with $\alpha$ subunits in yellow and $\beta$ subunits in pink (left, PDB entry: 2HHB) and sperm whale myoglobin (right PDB entry: $1 \mathrm{~A} 6 \mathrm{~N}$,). The, heme group is shown in red.

Unlike hemoglobin, myoglobin is a monomeric protein with a molecular weight around $16 \mathrm{kDa}$. This protein is mainly expressed in the striated muscle (skeletal muscle and heart) but $\mathrm{Mb}$ was also found in neurons, endothelial, smooth muscle, and tumor cells (Burmester \& Hankeln, 2014). Myoglobin exhibits one order of magnitude higher 
affinity for oxygen than hemoglobin. In the venous blood, where the concentration of $\mathrm{O}_{2}$ is low, only half of the hemoglobin will be $\mathrm{O}_{2}$ saturated while over $90 \%$ of myoglobin will be in $\mathrm{O}_{2}$ bound form this allows the myoglobin to extract $\mathrm{O}_{2}$ from the blood when hemoglobin release them (Voet \& Voet, 2010). Similar to Hb, Mb was also found to scavenge NO in its oxygenated form. On the other hand, deoxy Mb can produce NO by converting nitrite ion that can suppresses the reactive oxygen species (ROS) producing mitochondrial electron transport chain and subsequently protects against tissue damage caused by ischemia (Hendgen-Cotta et al., 2008). In addition, myoglobin can mediate hypoxic vasodilatation which is independent of the nitric oxide synthase pathway (Totzeck et al., 2012). Nevertheless, Mb-knockout mice did not show obvious physiological defects and exhibited normal exercise capacity due to the several physiological compensatory mechanisms, such as the increase of the hematocrit, that ensure the mouse survival (Garry et al., 1998). Importantly, Mb's mRNA and protein expression are significantly upregulated in the tumor cells (such as breast, prostate, and colon) which imply its possible role as a biomarker for cancer diagnose (Flonta et al., 2009; Gorr et al., 2010).

\subsection{Novel vertebrate globins}

In the last century, hemoglobin and myoglobin were considered the only globins in vertebrates. However, in the last two decades, with the help of sequencing of expressed sequence tags, six other globin types have been discovered in vertebrates: neuroglobin (Ngb), cytoglobin (Cygb), globin X (GbX), globin Y (GbY), globin E or 
eye-globin (GbE) and androglobin (Adgb) (figure 1.3)(Burmester \& Hankeln, 2014).

While the globin fold is conserved among vertebrate globins, most newly identified globins are different in taxa and tissue distribution. Globin $\mathrm{X}$ is found in "lower" vertebrate such as amphibians, fish, and reptiles but missing in bird and mammals, its expression patterns varies significantly across different species (Blank \& Burmester, 2012; Roesner et al., 2004). Globin Y is found in certain frogs (Xenopus), some lizard (Anolis carolinensis), some fish (Callobinchus milii) but is missing in the genomes of so called higher mammals such as Placentalia and Marsupialia or

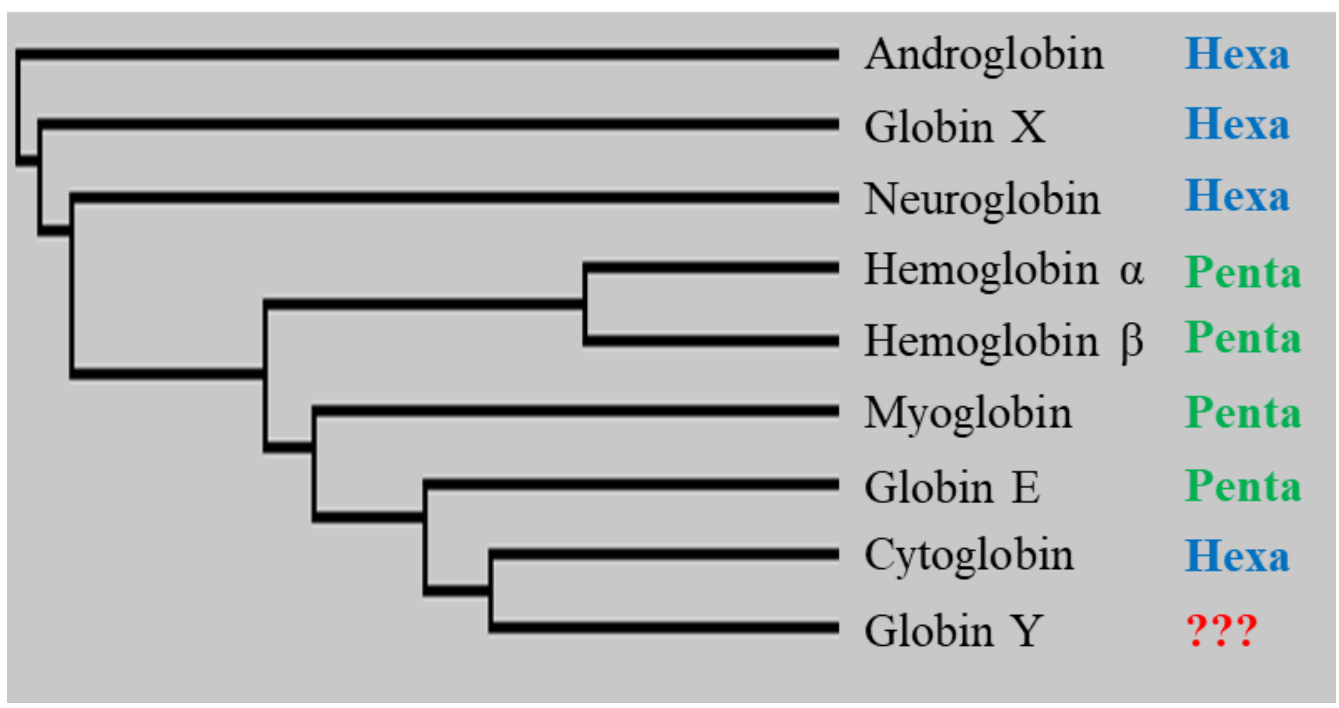

Figure 1.3. Simplified phylogenetic relationships in vertebrate globins by using uniport database along with their coordination type ( $\mathrm{GbY}$ is yet determined).

birds (Burmester \& Hankeln, 2014). On the other hand, GbE was first found in chicken and later discovered in the coelacanth. This protein was mainly expressed in the eye, suggesting its role in $\mathrm{O}_{2}$ supply to metabolically active retina (Blank et al., 2011). The least studied member of hexa-coordiante globoins is Adgb. This protein is widespread in Metazoan and predominantly expressed in testis suggesting its role in reproduction 
(Hoogewijs et al., 2011). So far, only a limited number of studies were performed on $\mathrm{GbX}, \mathrm{GbY}, \mathrm{GbE}$, and Adgb and their intracellular function and underlying molecular mechanism remain elusive. However, Cygb and Ngb have attracted more attention in the scientific community and their structural and physiological properties were studied intensively, as they have exhibit cellular protective and tumor suppression role in human and vertebrates (Bholah et al., 2015; Burmester \& Hankeln, 2009, 2014).

\subsection{Neuroglobin and Cytoglobin}

Neuroglobin is the first novel globin discovered by the Burmester group in 2000 (figure 1.4)(Thorsten Burmester et al., 2000). It is a small monomeric protein with a molecular mass around $17 \mathrm{kDa}$ and consist of $\sim 150$ amino acid residues. Neuroglobin is primarily expressed in neurons of the central and peripheral nervous systems and in the endocrine tissues (Laufs et al., 2004; Reuss et al., 2002). Neuroglobin can reversibly bind diatomic ligands and shown an oxygen affinity $\left(\mathrm{P}_{50}\right)$ of 1.9 to 2.3 torr, which is higher than that of mammalian $\mathrm{Hb}$ ( $\sim 26$ torr), but lower than that of $\mathrm{Mb}(\sim 1$ torr)(Dewilde et al., 2001).
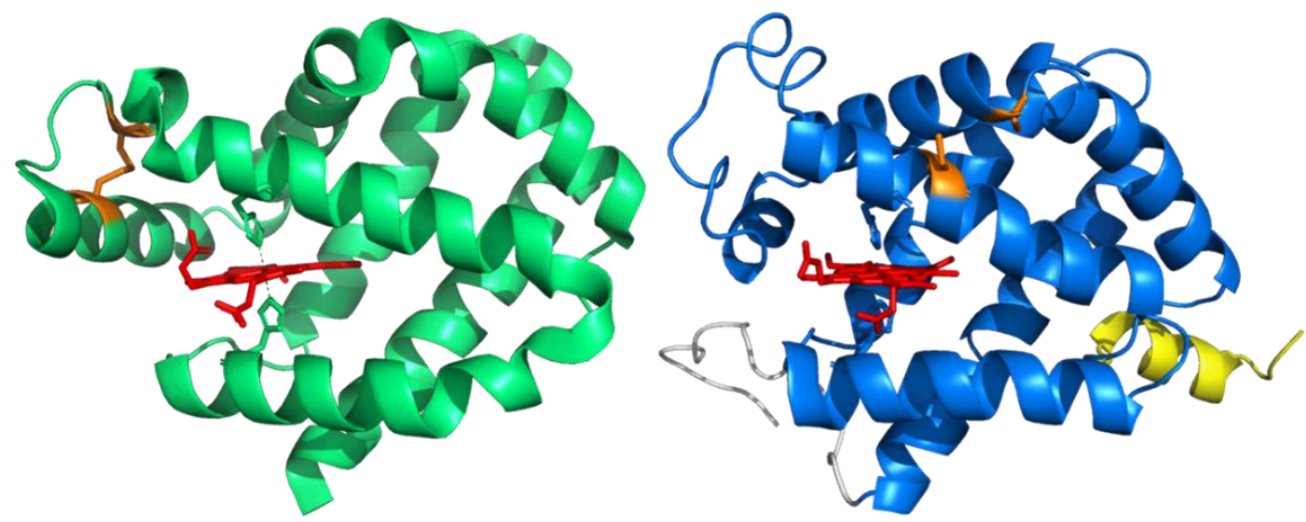

Figure 1.4. Ribbon representation of human neuroglobin (left, PDB: 4MPM chain A) and 
cytoglobin (right, remodeled by PDB: 2DC3). The proximal and distal histidine is shown in sticks, the heme is shown in red, two cysteine residues are in orange. The cytoglobin extended $\mathrm{N}$ terminal is shown in yellow and $\mathrm{C}$ terminal is shown in gray.

The crystal structure of $\mathrm{Ngb}$ reveals several unique structural characteristics as shown in figure 1.4. It has an internal disulfide bridge formed between two of its cysteine residues (Cys 35 and Cys 65) located in a loop between C and D $\alpha$-helices. In addition, the prosthetic group in Ngb is hexa-coordinated with distal histidine (His 64) binding to the heme iron in the oxidized (met form, $\mathrm{Fe}^{3+}$ ) and reduced (deoxy, $\mathrm{Fe}^{2+}$ ) form (Dewilde et al., 2001). Neuroglobin was initially considered to have similar function as $\mathrm{Mb}$ i.e. $\mathrm{O}_{2}$ transport. However, considering a low amount of $\mathrm{Ngb}$ expressed in neuronal tissue, additional physiological functions were proposed for this protein. In the cultured neurons, an overexpression of $\mathrm{Ngb}$ reduces superoxide anion generation after hypoxia/reoxygenation and improves neuron survival (Liu et al., 2009). In addition, transgenic mice over-expressing Ngb showed reduced cerebral and myocardial infarction after middle cerebral artery (MCA) occlusion induced ischemia suggesting a neuroprotective role of $\mathrm{Ngb}$ (Khan et al., 2006). It has been demonstrated that $\mathrm{Ngb}$ reduces cytochrome $\mathrm{c}$ which can prevent apoptosis since the ferrous Cyt $\mathrm{c}$ cannot trigger apoptosis (Fago et al., 2006). On the other hand, oxidized human Ngb $\left(\mathrm{Fe}^{3+}\right)$ binds the $\alpha$-subunits of heterotrimeric $\mathrm{G}$ proteins $(\mathrm{G} \alpha)$ and acts as a guanine nucleotide dissociation inhibitor (GDI) for Ga (Wakasugi et al., 2003). This also imply an anti-apoptosis role of $\mathrm{Ngb}$ because the inhibition of the dissociation of GDP from $\mathrm{G} \alpha$ and the release of $\mathrm{G} \beta \gamma$, protects cells from apoptosis induced death. Interestingly, 
$\mathrm{Ngb}$ is reported to interact with several other proteins such as voltage dependent anion channel (VDAC), cystatin C (a cysteine proteinase inhibitor), electron transferring flavoprotein alpha subunit (Etfa), Dvl1 (human homolog of the Drosophila disheveled gene), and flotillin-1 (a lipid raft microdomain-associated protein) pointing towards a potential role of $\mathrm{Ngb}$ in the cellular signaling pathways (Lechauve et al., 2009; Wakasugi, Nakano, \& Morishima, 2004; Wakasugi, Nakano, Kitatsuji, et al., 2004; Yu et al., 2012).

Unlike $\mathrm{Ngb}$, Cygb is a relatively large $(20.9 \mathrm{kDa})$ protein due to the presence of the extended $\mathrm{N}$ and $\mathrm{C}$ terminals. This protein is ubiquitously expressed at variable levels with no tissue specificity in both human and mice (Figure 1.3)(Nakatani et al., 2003; Trent \& Hargrove, 2002). To date, Cygb has been found in more than 20 tissues and cells such as thyroid, heart, adipose tissues, cervix, and coronary artery (Asahina et al., 2002; Thorsten Burmester et al., 2002). As a globin, Cygb is also able to reversible bind diatomic ligands such as $\mathrm{O}_{2}$ and compare to $\mathrm{Ngb}$, it has a high affinity for this gaseous ligand ( $\mathrm{P}_{50} \sim 1$ torr) which is similar to that found in $\mathrm{Mb}$, suggesting a potential role as a respiratory protein (Hamdane et al., 2003). However, as in other hexa-coordinate proteins, the exogenous ligands must compete with the distal histidine that coordinates heme iron. In addition, the low level of Cygb cellular concentration $(\sim 1 \mu \mathrm{M})$ and its localization make it less likely to have an analogous function as $\mathrm{Mb}$ ( $\mathrm{Li}$ et al., 2012). Like Ngb, overexpression of Cygb under hypoxic conditions increase cellular survival (Zhang et al., 2017). Nevertheless, reduced or eliminated expression of Cygb can 
hyperactivate a downstream effector of apoptosis, caspase-3, and promote cell death (Mathai et al., 2020). Also, several hypoxia responsive elements and hypoxia-inducible protein binding sites were found upstream of the Cygb gene, indicating a cellular protective role (Guo et al., 2007; Singh et al., 2009). As Ngb, Cygb was also reported to be involved in NO metabolism. A significant depression of cardiovascular functions, including a decrease in blood pressure and systemic vascular resistance, was observed in the mouse model with inactivated Cygb gene (X. Liu et al., 2017). This suggest that Cygb controls vascular reactivity through NO level regulation. Cygb expression is reduced in most cancer cells such as head and neck cancer, non-small cell lung cancers and a dramatic decrease (70\%) was reported for oesophageal cancer (McRonald et al., 2006; Oleksiewicz et al., 2011; Shivapurkar et al., 2008). Such expression suppression can be associated with the loss of heterozygosity and hypermethylation of $\mathrm{CpG}$ islands in the Cygb gene promoter region in the cancer cells (Mathai et al., 2020). Base on this, Cygb could have a putative role as a biomarker for cancer diagnosis. Cytoglobin knock out transgenic mice have shown tumorigenesis and multiple organ abnormalities in one to two-year-old mice that indicate Cygb as a critical fundamental protein which maintain normal cellular activity of life (Thuy et al., 2016). Last but not least, Cygb is reported to serve as an oxygen carrier for collagen synthesis while it is up-regulated after fibrotic damaged kidney and can inhibit several fibrosis-associated components, pointing towards itsanti-fibrosis function (Mathai et al., 2020). 


\subsection{GbX: a membrane-bound vertebrate globin}

$\mathrm{GbX}$ is a phylogenetically ancient type of globin first discovered in fish (Danio rerio) and amphibians (Xenopus) (figure 1.5)(Roesner et al., 2004). GbX shows 18\% to $26 \%$ sequence similarity with vertebrate $\mathrm{Mbs}, 22 \%$ to $26 \%$ similarity with $\mathrm{Cygb}$, and $15 \%$ to $25 \%$ similarity with vertebrate Hbs. Higher sequence similarity was reported for vertebrate $\mathrm{Ngbs}, 26.0 \%$ to $34.6 \%$ (Roesner et al., 2004).

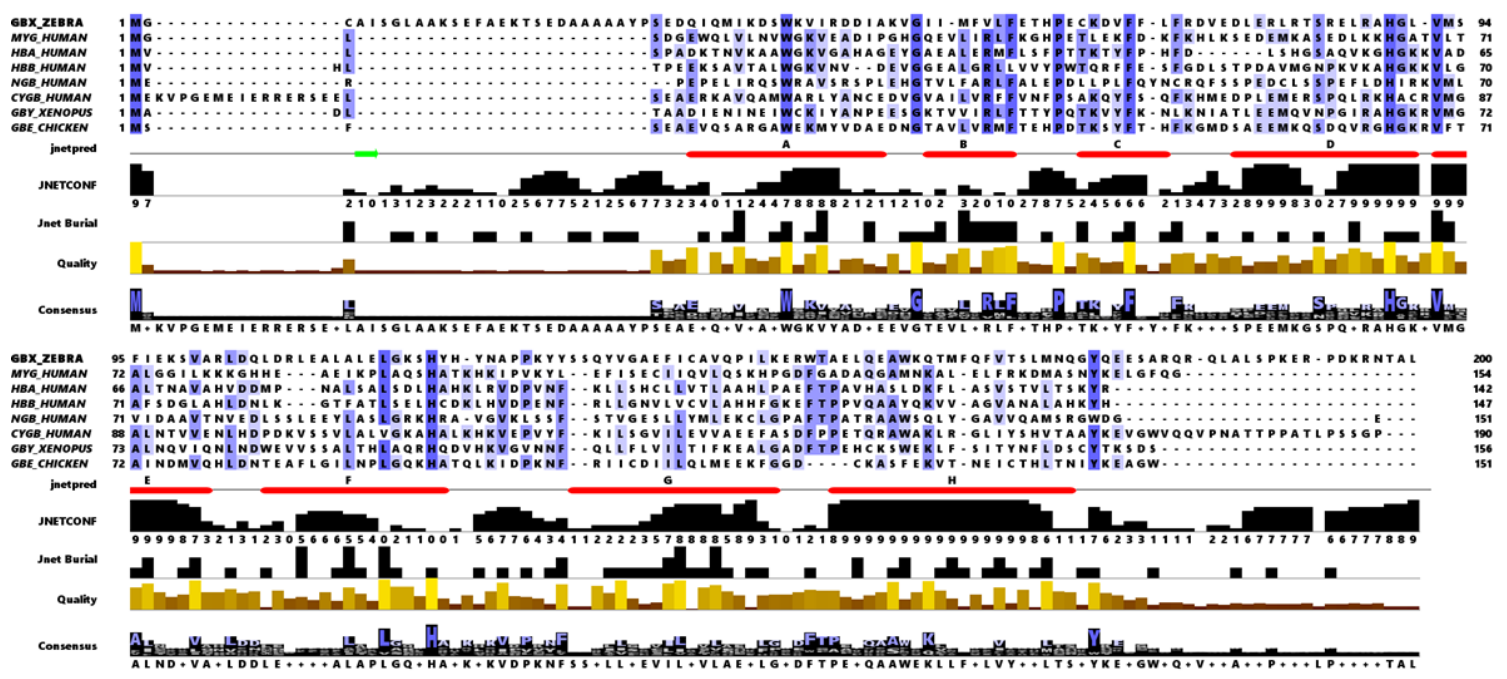

Figure 1.5. GbX sequence alignment with vertebrate globins and predicted secondary structures. Highly conserved amino acid residues are labeled with blue shadows.

The sequence of the globin core of GbX is highly conserved, with amino acid substitution rates as low as those observed in Ngb and Cygb. Therefore, the ancient divergence of $\mathrm{GbX}$ is probably the reason for the low similarity of $\mathrm{GbX}$ to other globins.

Globin $\mathrm{X}$ has been identified in many metazoan animals while it is missing in birds and mammals (T Burmester \& Hankeln, 2014). The distribution patterns of GbX across different species varies significantly, for example, it is widely expressed in goldfish tissues but more restricted in the brain and eye of Xenopus (Fuchs et al., 2006; Roesner et al., 2004). Burmester's group reported that GbX is the only vertebrate globin that has 
the ability to bind to cell membrane. Gly2 and Cys3 were identified as the myristoylation and palmitoylation sites on the N-terminus of $\mathrm{GbX}$ and the posttranslational acylation was proposed to be responsible for its cell membrane association capacity. Indeed, the removal of both residues, Gly2 and Cy3, abolishes the membrane association of GbX (Blank, Wollberg, et al., 2011). Subsequently, the impact of GbX on the survival of neuronal cells under hypoxic conditions or hydrogen peroxide induced stress was tested by Burmester's group in $\mathrm{GbX}$ and $\mathrm{Mb}$ transfected mouse neuron cells. Although both proteins, $\mathrm{Mb}$ and $\mathrm{GbX}$, enhanced cell viability under hypoxia, only acylated $\mathrm{GbX}$ efficiently protected cells against $\mathrm{H}_{2} \mathrm{O}_{2}$-induced stress. The loss of acyl group diminished the efficiency of neuronal protection, indicating that the protective role of $\mathrm{GbX}$ against ROS-stress requires the protein association to the cell membrane (Koch \& Burmester, 2016). Recently, Dr. Gladwin's group showed that GbX functions as a nitrite reductase with the catalytic constant of $26.7 \pm 2.0 \mathrm{M}^{-1} \mathrm{~s}^{-1}$ which is 5 to 10 fold higher than the value measured for R-state $\mathrm{Hb}$ and $\mathrm{Mb}, 25$ to 50 fold higher than the value observed in Ngb and Cygb, and even 200 fold higher than the value reported for T-state of $\mathrm{Hb}$ (Corti et al., 2016). The same group also reported that the recombinant $\mathrm{GbX}$ inhibits platelet aggregation more effectively than human $\mathrm{Hb}$. In addition, cultured fish RBCs can prevent platelet activation in the presence of nitrite whereas knockdown of $\mathrm{GbX}$ in the RBCs will render this capability. 


\subsection{Heme coordination}

In globins, the heme prosthetic group is located in the center of the typical 3 over 3 alpha helix structure (T Burmester \& Hankeln, 2014). Although the heme propionate groups form hydrogen bonds or salt bridges with nearby amino acid residues, the most important interaction between the heme and protein matrix is the heme-histidine coordination bond (Figure 1.6). The proximal histidine located on the F helix is highly conserved among all globins and this residue binds heme iron at fifth-coordination site. When there is no other internal amino acid residue in the position of the sixthcoordination site of the heme, this type of globin is named penta-coordinate globin as seen in $\mathrm{Hb}$ and $\mathrm{Mb}$. However, novel globins found in vertebrates (such as Cygb, Ngb, GbX, and Adgb) have additional distal histidine residue that coordinates to the heme iron in the sixth coordination site, forming the hexa-coordination ( $\mathrm{T}$ Burmester \& Hankeln, 2014).
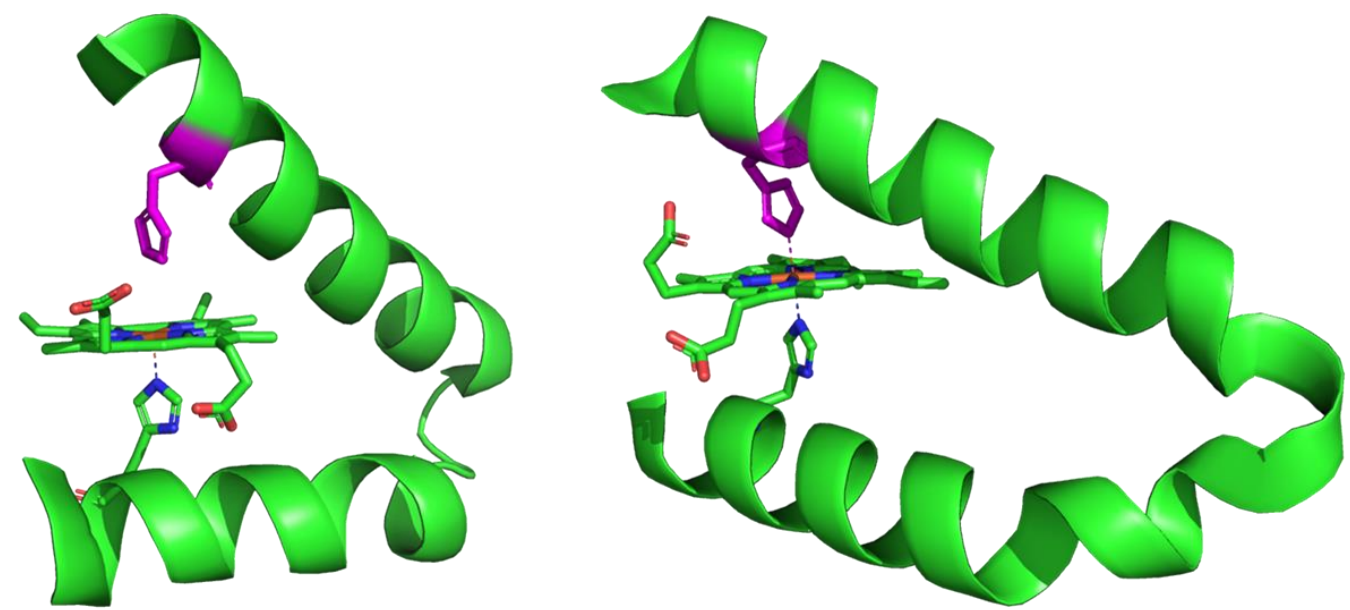

Figure 1.6. Ribbon representation of the heme binding site in vertebrate Mb (left, PDB entry $1 \mathrm{~A} 6 \mathrm{~N}), \mathrm{Ngb}$ (right, PDB entry 4MPM) demonstrating penta-coordination and hexacoordination respectively, of the heme iron. The distal (magenta) and proximal (green) histidine residues are shown as sticks. 
The hexa-coordination of the heme iron found in some vertebrate globins impacts the protein interactions with exogenous ligands, as the exogenous ligand such as $\mathrm{O}_{2}$ has to compete with distal histidine in order to bind to the heme and the binding rate of the exogenous ligand is limited by the rate of the dissociation of the distal histidine from the heme iron. The hexa-coordination is also found in the globins of other taxa such as non-symbiotic plant, bacterial and invertebrate, however, the exact physiological function of these globins is still unclear (Kakar et al., 2010). Surprisingly, phylogenetic analysis of the globin superfamily indicates that penta-coordinate vertebrate globins $(\mathrm{Mb}, \mathrm{Hb}$, and $\mathrm{GbE})$ possibly evolved from a hexa-coordinate ancestor because of the increasing demand of $\mathrm{O}_{2}$ supply (Blank \& Burmester, 2012). Interestingly, an unfolding study of $\mathrm{Ngb}$ and $\mathrm{Cygb}$ have shown an increased stability of both proteins compared to $\mathrm{Mb}$ implying that hexa-coordination of the heme iron might be a key feature in keeping the heme moiety bound to the globin structure at low pH (Picotti et al., 2009).

\subsection{Disulfide bridge modulate protein-ligand interaction and protein} stability

Other than hexa-coordination, Ngb, Cygb and GbX share another unique structural feature. Cys residues in Ngb (Cys46 and Cyg55), Cygb (Cys38 and Cys83) as well as GbX (Cys 65 and Cys 141) were reported to form internal and external disulfide bridges. Ngb and GbX are likely to adopt a monomeric form in the cell but formation of dimers with an intermolecular disulfide bridge connecting individual monomers was observed in SDS-PAGE electrophoresis and analytical ultra-centrifugation studies 
(Blank, Wollberg, et al., 2011; Dewilde et al., 2001). Monomeric dimeric, and tetrameric forms were observed in studies of Cygb, but only monomeric form was detected in the SDS-PAGE electrophoresis under reducing conditions, indicating that Cygb oligomers are stabilized through intermolecular disulfide bridges (Beckerson et al., 2015; Tsujino et al., 2014). However, there is no evidence to support that Cygb is in the form of a dimer or tetramer under physiological conditions.

The role of the internal disulfide bridges in vertebrate hexa-coordinate globins reminds unclear, however, considering relatively large distance between the Cys residues, the oxidation/reduction of the thiol groups trigger conformational changes in the structure of hexa-coordinate proteins which modulate the protein-ligand interactions. Namely, breakage of the disulfide bridge decreases the affinity of $\mathrm{O}_{2} 2$ fold in case of Cygb, two to four fold in case of $\mathrm{GbX}$, and ten fold in case of $\mathrm{Ngb}$ (Blank, Wollberg, et al., 2011; Hamdane et al., 2003). Also, the formation of an internal disulfide bridge is critical for the interaction between Cygb and lipids as well as its lipid-peroxidase activity since Cygb binds lipids in the presence of an intramolecular disulfide bridge (Beckerson et al., 2015).

In addition, the disulfide bridge is also involved in maintaining protein stability. The hexacoordinate globins shows an increased thermal stability with Cygb having melting temperature of $95^{\circ} \mathrm{C}$ and $\mathrm{Ngb}$ of $100^{\circ} \mathrm{C}$ whereas horse heart $\mathrm{Mb}$ has melting temperature of $81^{\circ} \mathrm{C}$ and human $\mathrm{Hb}$ of $71^{\circ} \mathrm{C}$ (Hamdane et al., 2005). Also, engineered human $\mathrm{Ngb}$ with one additional disulfide bridge showed enhanced tolerance 
to denaturant or acid induced protein unfolding as well as increased melting temperature (H.-X. Liu et al., 2019).

\subsection{Distal histidine: gate of the distal pocket}

Previous studies on pentacoordinate heme proteins have shown a crucial role of distal histidine residue in regulating affinity and kinetics of diatomic ligand binding to heme iron. A hydrogen bond between the distal histidine nitrogen and $\mathrm{O}_{2}$ bound to the heme iron stabilizes the gaseous ligand and prevents auto-oxidation of heme iron in both myoglobin and hemoglobin (Olson et al., 1988). Substitution of Gly for the distal histidine leads to a dramatic decrease in oxygen affinity caused by a 100 -fold increase in the $\mathrm{O}_{2}$ dissociation rate constant (Olson et al., 1988). Surprisingly, the distal histidine can function quite differently between penta-coordinate and hexa-coordinate globins. Distal histidine mutations in $\mathrm{Mb}$ induce up to 1000-fold increase in its autoxidation rate while mutation of the distal histidine improved $\mathrm{Fe}^{2+}-\mathrm{O}_{2}$ complex stability in $\mathrm{Ngb}$ around three fold (Tejero et al., 2015). Also, distal histidine replacement in Mb caused a 15fold decrease in the nitrite reduction rate compared to the wild type whereas the mutation of the distal histidine in Ngb increased the nitrite reduction rate 2000 -fold compare to wild type (Tejero et al., 2015). However, Cygb exhibits a similar redox potential $(20 \mathrm{mV})$ to $\mathrm{Mb}(46 \mathrm{mV})$ and similar of equilibrium constant for $\mathrm{O}_{2}$ and $\mathrm{CO}$ binding $\left(1 \mu \mathrm{M}^{-1}\right.$ and $21.7 \mu \mathrm{M}^{-1}$ for Cygb binding of $\mathrm{O}_{2}$ and $\mathrm{CO}$, respectively, and 1.1 $\mu \mathrm{M}^{-1}$ and $25.5 \mu \mathrm{M}^{-1}$ for $\mathrm{Mb}$ binding of $\mathrm{O}_{2}$ and $\mathrm{CO}$, respectively) pointing towards a similar function of the distal histidine in these proteins (Sawai et al., 2003). In addition, 
distal histidine in Cygb is critical for its peroxidase activity as distal histidine mutant exhibit no significant capability to oxidize guaiacol compare to wild type (Beckerson, Svistunenko, et al., 2015).

Distal histidine gating is observed in both hexa-coordinate and pentacoordinate globins as a mechanism of ligand migration regulation. The dissociation of the distal histidine is the rate limiting steps for ligand binding to heme iron in the hexacoordinate globins because the exogenous ligand has to compete with the distal histidine for iron coordination. The sidechain of the distal histidine that can be either oriented towards the solvent (so called open conformation) or be located in the distal pocket (so called close conformation). Both positions of the distal histidine were reported for Ngb and Cygb (Exertier et al., 2019; Makino et al., 2011). Similar open and close conformation of the distal histidine were also found in $\mathrm{Mb}$ and $\mathrm{Hb}$, and the gating effect of the distal histidine can be enhanced or weakened by replacing distal histidine with amino acid residues which has bigger bulky side chain or small shorter side chain, respectively (Birukou et al., 2010; Rohlfs et al., 1990).

Besides regulating the protein-ligand interaction, distal histidine can also play a role in modulating the stability of the globin. The hexa-coordinate Ngb and Cygb showed hyperthermal stability compared to the penta-coordinated $\mathrm{Mb}$ and $\mathrm{Hb}$ and this hyperthermal can be rendered if the distal histidine is mutated (Hamdane et al., 2005). In addition, the $\mathrm{pH}$-dependent heme release experiment demonstrates the midpoint for the heme from $\mathrm{Ngb}$ at $\mathrm{pH}$ of 3.2 which is 1.4 unit lower than for $\mathrm{Mb}, \mathrm{pH}_{1 / 2}=4.6$. 
However, replacement of distal histidine with glutamine in Ngb leads to an increase of $\mathrm{pH}_{1 / 2}$ to 4.5 (Picotti et al., 2009).

\subsection{Extended N- and C-terminals}

Cytoglobin has extended $\mathrm{N}$ terminal by 18 amino acid residues and $\mathrm{C}$ terminal by 16 amino acid residues. The crystal structure revealed that the $\mathrm{N}$ terminal, which is highly charged with four positively and seven negatively charged residues, forms a nine amino acid residues long alpha helix whereas the $\mathrm{C}$ terminal, which contains five proline residues, adopts a random coil (Makino et al., 2006). While both N- and Cterminals showed no influence on Cygb lipid binding, C- terminal was proposed to affect Cygb's superoxide-scavenging activity and protein stability (Hanai et al., 2017). Since the N-terminal extension in Cygb adopts the alpha helix, a gas sensing function was proposed for this protein, based on similarity with the heme-based aero taxis transducer (HemAT) from Bacillus subtilis which exhibits a globin fold with the pre-A helix (Hou et al., 2000).

In addition, another vertebrate globin, $(\mathrm{GbX})$ was also reported to have $\mathrm{N}$ - and C- terminal extensions. Two acylation sites were found on the $\mathrm{N}$ terminal of GbX and GbX acylation promotes its association to the cell membrane. But the $\mathrm{C}$ terminal extension function is unknown (Blank, Wollberg, et al., 2011). So, the physiology role of the extended $\mathrm{N}$ - and $\mathrm{C}$-terminals needs to be determined in the novel vertebrate globins. 


\subsection{Heme disorder and sliding.}

Two orientations of the prosthetic group in human Ngb and mouse Ngb have been reported by NMR and X-ray crystallography as well as in molecular dynamics simulation studies (Arcovito et al., 2008; Bocahut et al., 2012; Du et al., 2003; Xu et al., 2009). In $\mathrm{Ngb}$, the heme group can rotate $180^{\circ}$ around the $\alpha, \gamma$-meso axis, with both propionate group exposed to the solvent which is different from the unique heme orientation in $\mathrm{Mb}$ and $\mathrm{Cgb}$ (figure 1.7). Such heme orientation disorder is facilitated by the enlarge heme distal cavity in Ngb and may impact the protein-ligand interaction. The putative mechanism of how the heme orientation disorder in Ngb impacts the ligand association rate was proposed by Bocahut et al. Interactions between the vinyl and methyl group on the porphyrin ring and the non-polar amino acid residues (such as, Val 109, Val 68) provide a steric hinder for the ligand migration pathway that links the distal pocket with the solvent and ultimately reduces the rate for ligand association to the heme iron (Bocahut et al., 2012). In addition, based on X-ray crystallography data, so called heme sliding phenomenon in the $\mathrm{CO}$ bond murine $\mathrm{Ngb}$ was reported (Vallone et al., 2004). While overall structure of Ngb is moderately affected by CO binding, the heme prosthetic group slides deeper into the distal cavity in the $\mathrm{CO}$ bound protein (figure 1.8). In addition, the large hydrophobic tunnel connecting the distal pocket and proximal cavity disappears after $\mathrm{CO}$ binding of $\mathrm{Ngb}$. It was proposed that the heme 
sliding into the deeper distal pocket can destabilize the distal histidine and thus regulate the exogenous ligand binding affinity to Ngb (Vallone et al., 2004). Several amino
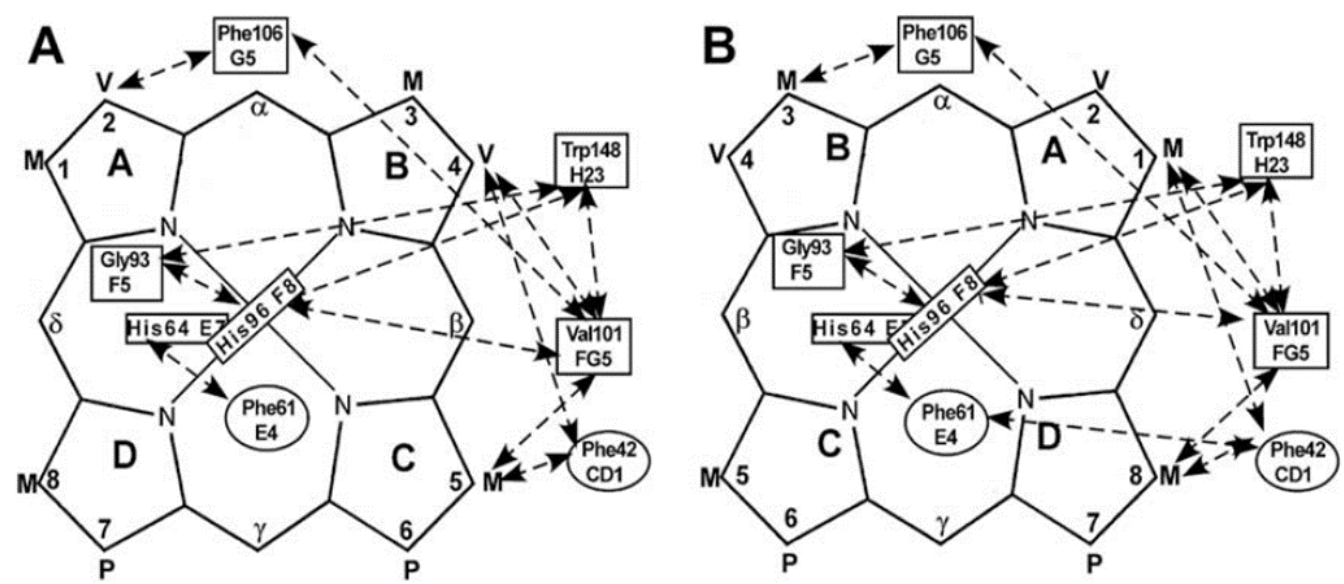

Figure 1.7. The heme orientations in $\mathrm{Mb}(\mathrm{A})$ and rotated $180^{\circ}$ about the $\alpha-\gamma$-meso axis (B). The heme substituents are labeled M (methyl), V (vinyl), and $\mathrm{P}$ (propionate) (Xu, Li, et al., 2009). acid residues were proposed to play a role in heme sliding but only Phe-106 changes its position upon heme sliding to its crevice. NMR and molecular dynamic simulation study have shown that the Phe-106 belongs to the key residues which maintain heme stability inside the distal cavity (Bocahut et al., 2012; Xu, Yin, et al., 2009). Indeed, mutating Phe-106 will trigger the rearrangement of the heme environment and induce further displacement of the heme. 


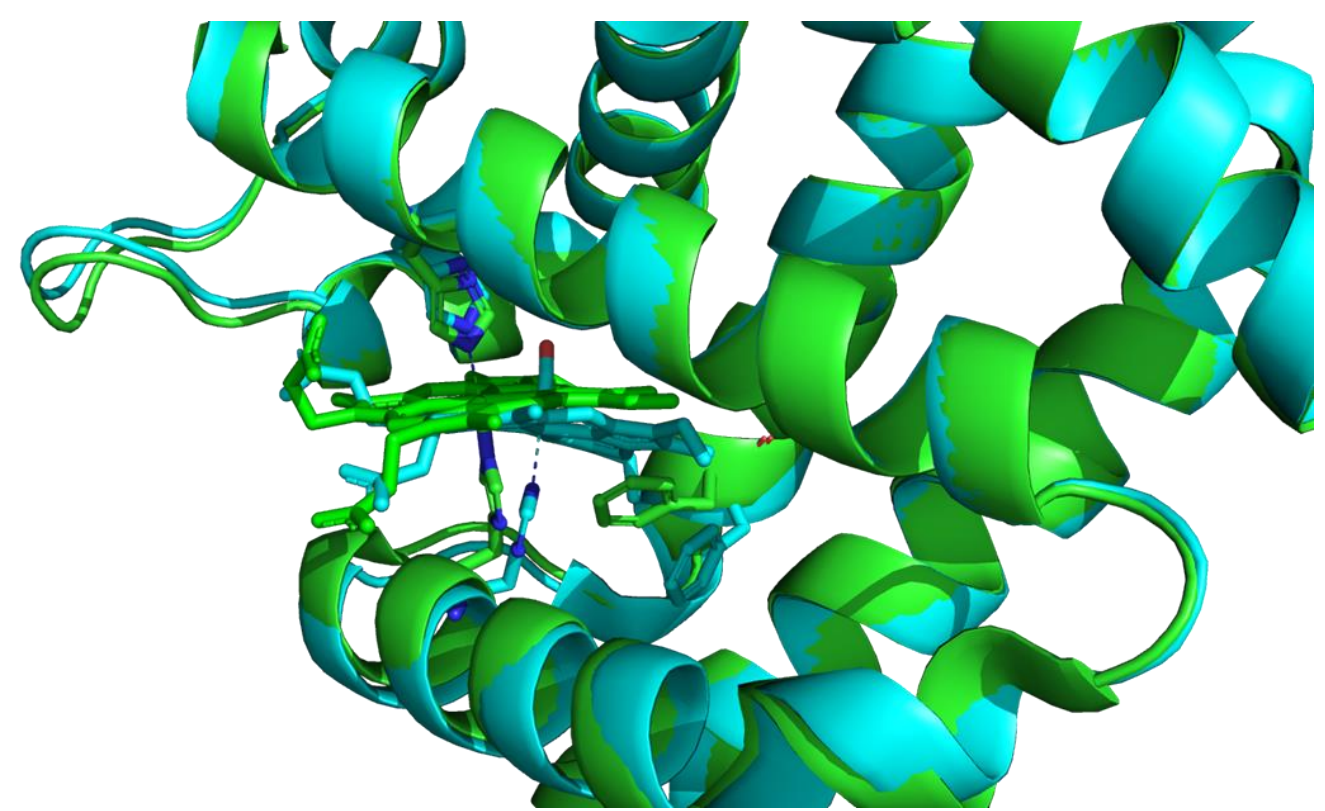

Figure 1.8. Heme sliding upon CO binding. Ferric murine Ngb (in green, PDB: 5EET), CO bound murine Ngb (in cyan, PDB: 1W92). Distal and proximal histidine, heme group as well as Phe-106 are also shown as sticks.

\subsection{Globin interactions with exogenous ligands}

Globins reversibly bind various exogenous ligands especially diatomic gaseous ligand such as $\mathrm{O}_{2}, \mathrm{CO}, \mathrm{NO}$ in it reduce form $\left(\mathrm{Fe}^{2+}\right)$. Also, globins can interact with other ligands in its met form $\left(\mathrm{Fe}^{3+}\right)$ such as $\mathrm{CN}$ - and $\mathrm{H}_{2} \mathrm{~S}$. Characterization of interactions between globins and exogenous ligands in terms of the equilibrium affinity constant, individual rate constants and associated thermodynamic parameters, enthalpy, entropy and volume changes provide valuable information that helps to reveal potential functions of the globins, such as $\mathrm{O}_{2}$ transportation and storage, peroxidase activity or NO reductase etc. In addition, understanding of heme proteins interactions with toxic ligands provide insight into the molecular mechanism of toxicity. For example, $\mathrm{CO}$ is toxic because it can tightly bind to hemoglobin thereby restrict $\mathrm{O}_{2}$ supply in the body. Therefore, study of the interaction between globins and exogenous ligands is important 
to reveal their physiological functions and understand the molecular mechanisms of their functions. 


\section{OBJECTIVES}

Characterization of structural dynamic in protein is critical for understanding of the structural mechanism that modulate the protein interactions with ligand, binding partners and most importantly to understand their physiological functions. In addition, the studies of protein dynamics in combination with site directed mutagenesis provide an elegant approach to characterize the role of individual amino acid residues in controlling the affinity and kinetics of ligand binding to proteins. In addition, determination of thermodynamic parameters, such as activation and reaction enthalpy, entropy and volume change for ligand association and dissociation, provide insight into energetics of protein ligand interactions. In this work, structural dynamics associated with ligand binding to novel vertebrate globins, namely GbX, were investigated using steady-state and time-resolved spectroscopic methods. Beside to understanding the interactions of vertebrate globins with $\mathrm{CO}$ and $\mathrm{O}_{2}$, a part of this study was devoted to characterizing how can conformation of the protein affect the ligand accessibility to the protein distal pocket by using heme florescent analogs. The universal goal of this study is to provide molecular insight into the mechanism of ligand migration and interactions with vertebrate globins.

In chapter 4, transient absorption spectroscopy as well as photoacoustic calorimetry was employed to characterize $\mathrm{CO}$ association and dissociation to $\mathrm{GbX}$, in order to determine the reactivity and heterogeneity of heme iron in hexacoordinate globins. Also, disulfide bridge and distal histidine mutants were employed to study 
how these distinct characteristics impact ligand binding, ligand affinity and structural stability of $\mathrm{GbX}$.

In chapter 5, Cygb, Ngb and their mutant were reconstituted by zinc protoporphyrin IX which is a florescent analog of iron protoporphyrin IX (native heme). Quenching study were performed by using the reconstituted florescent protein. This study provides information on conformational heterogeneity in hexacoordinate globins as well as on the heme pocket accessibility.

In chapter 6 , transient absorption spectroscopy as well as photoacoustic calorimetry was employed to characterize $\mathrm{O}_{2}$ association and dissociation to $\mathrm{GbX}$ as well as acid titration was employed to determine how the stability of the protein is impacted by exogenous ligand binding to the heme iron. 


\section{MATERIAL AND METHODS}

\subsection{Materials}

The Zinc protoporphyrin IX, $\mathrm{Fe}^{3+}$ tetrakis(4-sulfonatophenyl)phorphine [Fe(III)4SP] was purchased from Frontier Scientific Inc. Myoglobin, dithiothreitol (DTT), Sodium dithionite, Sodium cyanide $(\mathrm{NaCN})$, and 5- $\delta$ - aminolevulenic acid (5ALA) were purchased from Sigma-Aldrich. All other reagents were purchased from Fisher Scientific. All reagents were used as received without further purification.

\subsection{Methods}

\subsubsection{Protein expression and purification.}

Cells transformed with plasmids for wild type human Ngb, human Cygb, Zebra fish GbX and mutants were kindly provided by Dr. Pierre Sebban (Paris Sud University, Orsay, France). A six-His tag was adding into the $\mathrm{N}$ terminal of the coding sequence of Cygb, Ngb and GbX then cloned into a pET15b expression vector. DNA sequencing was performed to confirm the correction of the insertion of the express DNA sequence in the plasmid. Escherichia coli strain BL21 was used for transforming the expression vectors and expression of recombinant proteins

\subsubsection{Neuroglobin and Cytoglobin isolation and purification.}

A pre-culture of $50 \mathrm{~mL}$ of Terrific Broth medium was cultured with the $\mathrm{Ngb}$ or Cygb cell for $6 \mathrm{~h}$ at $37^{\circ} \mathrm{C} 225 \mathrm{rpm}$ with $100 \mathrm{mg} \mathrm{L}^{-1}$ of ampicillin. Next, $15 \mathrm{~mL}$ of the pre-culture was transferred into $1 \mathrm{~L}$ of Terrific Broth medium with $100 \mathrm{mg} \mathrm{L}^{-1}$ of ampicillin and $170 \mathrm{mg} \mathrm{L}^{-1}$ of 5-aminolevulenic acid. Cells were incubated at $37^{\circ} \mathrm{C}$ and 
$225 \mathrm{rpm}$, until $\mathrm{A}_{600} \mathrm{~nm}$ reached 0.8 and the protein expression was induced by addition of IPTG to a final concentration of $0.4 \mathrm{mM}$. Cells were then incubated overnight at $30^{\circ} \mathrm{C}$ and $170 \mathrm{rpm}$. Cell pellet was collected by centrifugation for $15 \mathrm{~min}$ at $5000 \mathrm{rpm}$ and $4{ }^{\circ} \mathrm{C}$ (Thermo Fisher 40R) and homogenized in $50 \mathrm{mM}$ Tris buffer with $1 \mathrm{mM}$ DTT, $1 \mathrm{mM}$ PMSF and 1mM EDTA (pH 8.0). The suspension was sonicated with a sonic dismembrator (Model 100, Fisher Scientific) and centrifuged at 5,000 rpm to remove membrane debris. The supernatant was filtered through a $0.45 \mu \mathrm{m}$ membrane and loaded into a Ni-NTA column (Qiagen) that was previously equilibrated with 5 $\mathrm{mM}$ Tris buffer ( $\mathrm{pH}$ 8.0). $50 \mathrm{mM}$ Tris buffer with an increasing imidazole concentration of 5-20 mM was used to wash the column to remove non-specifically bound proteins. The column was washed until the $280 \mathrm{~nm}$ absorbance of eluate was less than 0.01. Ngb or Cygb was eluted with $5 \mathrm{mM}$ Tris buffer $(\mathrm{pH} 8.0)$ containing 40 $\mathrm{mM}$ imidazole. Fractions with an $\mathrm{A}_{\text {Soret }} / \mathrm{A}_{280 \mathrm{~nm}}$ ratio higher than 2.4 were collected and concentrated using Amicon Millipore concentrators. Purified protein was then dialyzed overnight against $50 \mathrm{mM}$ Tris buffer (pH 7.0). SDS-Page electrophoresis and UV-vis spectroscopy were employed to assess the protein purity and protein was stored at $-40{ }^{\circ} \mathrm{C}$.

\subsubsection{Globin $X$ isolation and purification}

A pre-culture of $50 \mathrm{~mL}$ of Terrific Broth medium was cultured with the cell for $6 \mathrm{~h}$ at $37^{\circ} \mathrm{C} 225 \mathrm{rpm}$ with $100 \mathrm{mg} \mathrm{L}{ }^{-1}$ of ampicillin. Next, $15 \mathrm{~mL}$ of the pre-culture was transferred into $1 \mathrm{~L}$ of Terrific Broth medium with $100 \mathrm{mg} \mathrm{L}^{-1}$ of ampicillin as well as 
$170 \mathrm{mg} \mathrm{L}^{-1}$ of 5-aminolevulenic acid. Cells were incubated at $37^{\circ} \mathrm{C}$ and $225 \mathrm{rpm}$, until $\mathrm{A}_{600 \mathrm{~nm}}$ reached 1.2 then induced by addition of IPTG to a final concentration of 0.4 $\mathrm{mM}$ and incubated overnight at $30^{\circ} \mathrm{C}$ and $170 \mathrm{rpm}$. Cell pellet was collected by centrifugation for $15 \mathrm{~min}$ at $5000 \mathrm{rpm}$ and $4^{\circ} \mathrm{C}$ (Thermo Fisher $40 \mathrm{R}$ ) and homogenized in $50 \mathrm{mM}$ Tris buffer with $1 \mathrm{mM}$ DTT, $1 \mathrm{mM}$ PMSF and 1mM EDTA (pH 8.0). The suspension was sonicated with a sonic dismembrator (Model 100, Fisher Scientific) and centrifuged at 5,000 rpm to remove membrane debris. The supernatant was filtered through a $0.45 \mu \mathrm{m}$ membrane and loaded into a Ni-NTA column (Qiagen) that was previously equilibrated with $5 \mathrm{mM}$ Tris buffer ( $\mathrm{pH}$ 8.0). Gradient imidazole buffer (50 $\mathrm{mM}$ Tris, 5-40 mM imidazole) was used to wash the column to remove nonspecific binding protein until the absorbance at $280 \mathrm{~nm}$ was less than 0.01 . GbX was eluted with $5 \mathrm{mM}$ Tris buffer (pH 8.0) containing $100 \mathrm{mM}$ imidazole. Fractions with an $A_{\text {Soret }} / A_{280} \mathrm{~nm}$ ratio higher than 2.5 were collected and concentrated using Amicon Millipore concentrators. Purified protein was then treated with potassium ferricyanide to obtain the ferric form of $\mathrm{GbX}$ and further purified by $\mathrm{G} 25$ size-exclusion chromatography to remove potassium ferricyanide, following an overnight dialysis against $50 \mathrm{mM}$ Tris buffer $(\mathrm{pH}$ 7.0). SDS-Page electrophoresis and UV-vis spectroscopy were employed to assess the protein purity.

\subsubsection{Sodium dodecyl sulfate electrophoresis}

Sodium dodecyl sulfate (SDS) electrophoresis was performed according to the procedure described by Gallagher (Gallagher, 2001). Electrophoresis gels were 
purchased from NuSep (Tris-Glycine Precast Gels). The composition of 1L 10X running buffer is shown in Table 3.1. Protein samples for electrophoresis were prepared by mixing the protein solution with $20 \mu \mathrm{L}$ of loading buffer (Table 3.2). The protein samples were then boiled in a water bath for 8 minutes before loading to into the gel. In general, 10 to $20 \mu \mathrm{L}(\sim 5 \mu \mathrm{g})$ of protein solubilized in loading buffer were loaded into each well of the electrophoresis gel.

\begin{tabular}{|l|l|}
\hline Ingredient & Amount \\
\hline Tris-base & $30.3 \mathrm{~g}$ \\
\hline Glycine & $144.4 \mathrm{~g}$ \\
\hline SDS & $10 \mathrm{~g}$ \\
\hline
\end{tabular}

Table 3.1.Ingredient of 1L 10X SDS running buffer for electrophoresis.

Initially, voltage of $100 \mathrm{~V}$ was applied using a power supply (Model FB300, Fisher Scientific) for 15 mins to let the sample enter the gel and then the voltage was increase to $150 \mathrm{~V}$ until the $20 \mathrm{kDa}$ marker reaches $1 \mathrm{~cm}$ above the bottom of the gel.

\begin{tabular}{|l|l|}
\hline Ingredient & Amount \\
\hline Tris-HCl & $0.3 \mathrm{M}$ \\
\hline DTT & $0.6 \mathrm{M}$ \\
\hline SDS & $10 \%$ \\
\hline Glycerol & $30 \%$ \\
\hline Bromophenol blue & $<0.06 \%$ \\
\hline
\end{tabular}

Table 3.2. Ingredient of loading buffer for electrophoresis.

Upon completion of the electrophoresis, the gel was removed from the plastic frame and stained by Coomassie blue staining buffer for at least 3 hours or overnight on a low speed shaker. The gel was de-stained by de-staining buffer for 10 mins, then 


\begin{tabular}{|l|l|}
\hline Ingredient & Amount \\
\hline Methanol & $50 \%$ \\
\hline Acetic acid & $10 \%$ \\
\hline DI Water & $40 \%$ \\
\hline
\end{tabular}

Table 3.3. Ingredient of Coomassie blue staining buffer.

the colored de-staining buffer was exchange with the fresh de-staining buffer and destaining procedure was carry out for additional 2 hours. After de-staining, the gel was

\begin{tabular}{|l|l|}
\hline Ingredient & Amount \\
\hline Coomassie blue R-250 & $<0.1 \%$ \\
\hline Methanol & $20 \%$ \\
\hline Acetic acid & $10 \%$ \\
\hline DI Water & $69 \%$ \\
\hline
\end{tabular}

Table 3.4. Ingredient of de-staining buffer.

placed into de-ionized water until it fully relaxed then ready for imaging. The composition of the staining and the de-staining buffer is listed in Tables 3.3 and 3.4, respectively.

\subsubsection{UV-vis Spectroscopy}

Primarily as a quantitative analytical technique, Ultraviolet-visible (UV-vis) spectrophotometry is focused on the absorption of near-UV (180-390 nm) or visible (390-780 nm) radiation by chemical species in the solution (Worsfold \& Zagatto, 2005). The electromagnetic spectrum (Table 3.5) of these regions can provide energy 
that induce the electronic transitions. Different color of visible light will be absorbed and exhibit at particular wavelengths (Table 3.6).

The Beer's law describes the relationship between absorbance and concentration, and is defined by equation 3.1 :

$$
A=\varepsilon b c
$$

Where $\mathrm{A}$ is the absorbance of the sample (no unites), $\varepsilon$ is the molar absorptivity (1 $\left.\mathrm{mol}^{-1} \mathrm{~cm}^{-1}\right), b$ is the path length of the cell with the absorbing medium $(\mathrm{cm})$, and $\mathrm{c}$ is the concentration $\left(\mathrm{mol}^{-1}\right)$. Generally, organic chemicals, such as aromatic compound, absorb energy in the near-UV region as a result of $\sigma \rightarrow \sigma^{*}, n \rightarrow \sigma, n \rightarrow \pi^{*}$, and $\pi \rightarrow \pi^{*}$ transitions (Worsfold \& Zagatto, 2005). For biochemical samples, protein is strongly absorbed in the range of $180-240 \mathrm{~nm}$ with is correlated to $\mathrm{n} \rightarrow \pi^{*}, \pi \rightarrow \pi^{*}$ transitions of the peptide bond (Rosenheck \& Doty, 1961).

\begin{tabular}{|l|l|l|l|}
\hline Region & Wavelength range $(\mathrm{m})$ & $\begin{array}{l}\text { Wavelength range } \\
\text { (commonly used units) }\end{array}$ & Type of transition \\
\hline Far-Ultraviolet & $10^{-8}-1.8 \times 10^{-7}$ & $10-180 \mathrm{~nm}$ & Middle shell electron \\
\hline Near-Ultraviolet & $1.8 \times 10^{-7}-3.9 \times 10^{-7}$ & $180-390 \mathrm{~nm}$ & Outer shell electron \\
\hline Visible & $3.9 \times 10^{-7}-7.8 \times 10^{-7}$ & $390-780 \mathrm{~nm}$ & Outer shell electron \\
\hline Near-infrared & $7.8 \times 10^{-7}-2.5 \times 10^{-6}$ & $0.78-2.5 \mu \mathrm{m}$ & Molecular vibration \\
\hline Mid- and far-infrared & $2.5 \times 10^{-6}-10^{-3}$ & $2.5-1000 \mu \mathrm{m}$ & $\begin{array}{l}\text { Molecular vibration } \\
\text { and rotation }\end{array}$ \\
\hline
\end{tabular}

Table 3.5. The electromagnetic spectrum (Worsfold \& Zagatto, 2005).

The information of poly-L-glutamic acid absorption spectra under different conditions of ionic strength and $\mathrm{pH}$ can be used to estimate the secondary structure of a peptide. It is reported that random coil has an absorption maxima at $192 \mathrm{~nm}$ with a molar extinction coefficient around $7.1 \mathrm{mM}-1 \mathrm{~cm}-1$ while $\beta$-sheet conformations 
absorb maxima at $194 \mathrm{~nm}$ (Rosenheck \& Doty, 1961). Other than peptide bond, 3 amino acid residues with aromatic sidechains, tryptophan, tyrosine, and phenylalanine, absorb between $260-280 \mathrm{~nm}$ with molar extinction coefficient at $280 \mathrm{~nm}$ of $5.5 \mathrm{mM}^{-1} \mathrm{~cm}^{-1}$ (Trp), $1.49 \mathrm{mM}^{-1} \mathrm{~cm}^{-1}$ (Tyr), and $0.2 \mathrm{mM}^{-1} \mathrm{~cm}^{-1}$ (Phe)(Mach et al., 1992). In addition, porphyrin exhibit several absorption bands with high extinction coefficients. The Soret or B-band is located between $380-500 \mathrm{~nm}$ and has a molar extinction coefficient of $10^{5}$ $\mathrm{M}^{-1} \mathrm{~cm}^{-1}$. In the visible part of the spectrum, between $500-750 \mathrm{~nm}$, porphyrin exhibits several absorption bands, so called Q bands, with molar extinction coefficients of $10^{4}$ $\mathrm{M}^{-1} \mathrm{~cm}^{-1}$ (Uddin, 2012). Thus, the presence of the heme prosthetic group in proteins provides an extraordinary benefit to characterize the change in the heme iron spins, oxidation state or structural changes in the heme surroundings as the heme electronic spectrum is strongly impacted by these changes.

\begin{tabular}{|l|l|l|}
\hline Wavelength $(\mathrm{nm})$ & Color absorbed & Color observed \\
\hline $390-420$ & Violet & Green-yellow \\
\hline $420-440$ & Violet-blue & Yellow \\
\hline $440-470$ & Blue & Orange \\
\hline $470-500$ & Blue-green & Red \\
\hline $500-520$ & Green & Purple \\
\hline $520-550$ & Yellow-green & Violet \\
\hline $550-580$ & Yellow & Violet-blue \\
\hline $580-620$ & Orange & Blue \\
\hline $620-680$ & Red & Blue-green \\
\hline $680-780$ & Purple & Green \\
\hline
\end{tabular}

Table 3.6. The visible spectrum (Worsfold \& Zagatto, 2005).

\subsubsection{Circular Dichroism}

Circular dichroism (CD) spectroscopy is a spectroscopic technique that allows for characterization of interactions between molecules and circularly polarized light. 
Although CD spectroscopy is used widely in studies of small chiral molecules, it is an important spectroscopic technique to monitor protein secondary structure. In the CD spectroscopy, the absorbance differences between left $\left(A_{L}\right)$ and right $\left(A_{R}\right)$ circularly polarized light of the sample (Equation 3.2).

$$
\Delta A=A_{L}-A_{R}
$$

The signal is commonly recorded as ellipticity $(\theta)$ in degrees and plotted as a function of wavelength. The general relationship between $\Delta \mathrm{A}$ and $\theta$ is described by equation 3.3 (Kelly et al., 2005).

$$
\theta=32.98 \Delta A
$$

The CD signal can only be detected for a chiral chromophore. The chromophore is chiral if: (i) it has a chiral chemical structure, (ii) it is covalently connected with a chiral center in a molecule, or (iii) it is located inside an asymmetric environment of a macro-molecule (Kelly et al., 2005).

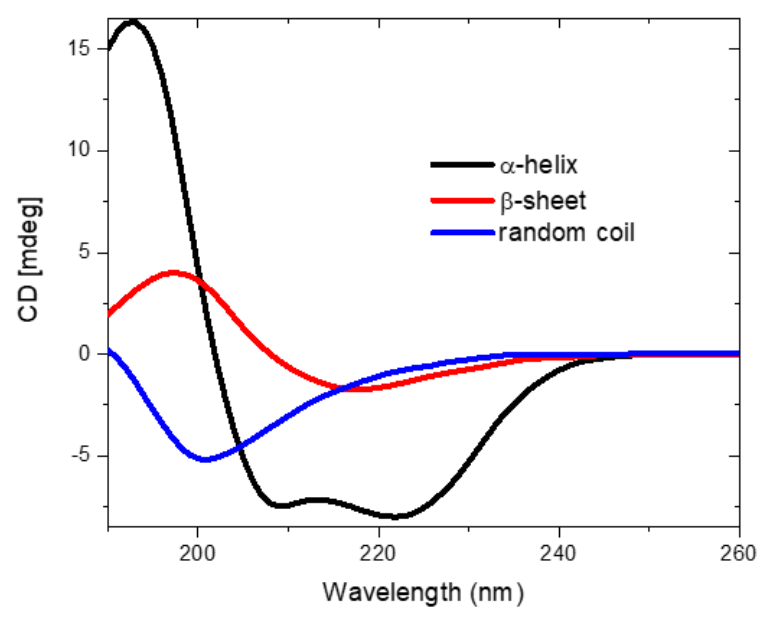

Figure 3.6. Sample electronic CD spectra of protein in $\alpha$-helix, a $\beta$-sheet and a random coil. Simulated by PDBMD2CD.com with data from protein data bank. 
In the protein, the optically active chromophores are the peptide bond absorbing below $240 \mathrm{~nm}$, amino acid residues with aromatic side chains that absorb around 260 to $320 \mathrm{~nm}$ and a disulfide bridge with broad absorbance centered around $260 \mathrm{~nm}$ (Kelly et al., 2005). Determination of the secondary structure of polypeptides and proteins is one of CD's major application in biochemistry, as different secondary structures have distinct CD spectra as shown in Figure 3.6. For example, a typical $\alpha$ - helix exhibits a negative peak at $\sim 222 \mathrm{~nm}$ and $208 \mathrm{~nm}$ together with a positive peak at $190 \mathrm{~nm}$ (Holde et al., 1998). On the other hand, $\beta$-sheet is characterized by a negative peak at $215 \mathrm{~nm}$ together with a positive peak at $\sim 198 \mathrm{~nm}$ (Holde et al., 1998). Random coil shows a negative peak at around $195 \mathrm{~nm}$. There is a decent number of algorithms and software packages which allow to quantify the secondary structure amount based on the far UV CD spectra (Drew et al., 2018). The prediction of the secondary structure of the protein is based on comparison of the CD spectrum of a given protein with the $\mathrm{CD}$ data for proteins with known secondary structure. However, the accuracy secondary structure prediction is limited, and the results should be use with cautious.

Importantly, ligand binding, sequence alteration as well as point mutations may induce secondary structure changes can be observed in the CD spectra and thus this technique can be used to characterize ligand interactions with proteins as well as impact individual amino acids on protein structure. In addition, CD spectroscopy is often used to monitor protein folding/unfolding as a function of temperature or $\mathrm{pH}$ and to 
determine the melting temperature or $\mathrm{pH}$ at half-transition that are important parameters for protein stability characterization.

\subsubsection{Transient absorption spectroscopy}

\subsubsection{Introduction}

Time-resolved techniques allows characterization of kinetic parameters for ligand binding and dissociation from proteins that are necessary to construct kinetic models and reveal the mechanism of the protein-ligand interaction. Stopped-flow is the pioneer of the time-resolved absorption-coupled techniques and was first employed to record kinetic data for hemoglobin-oxygen complex formation (Hartridge \& Roughton, 1923).
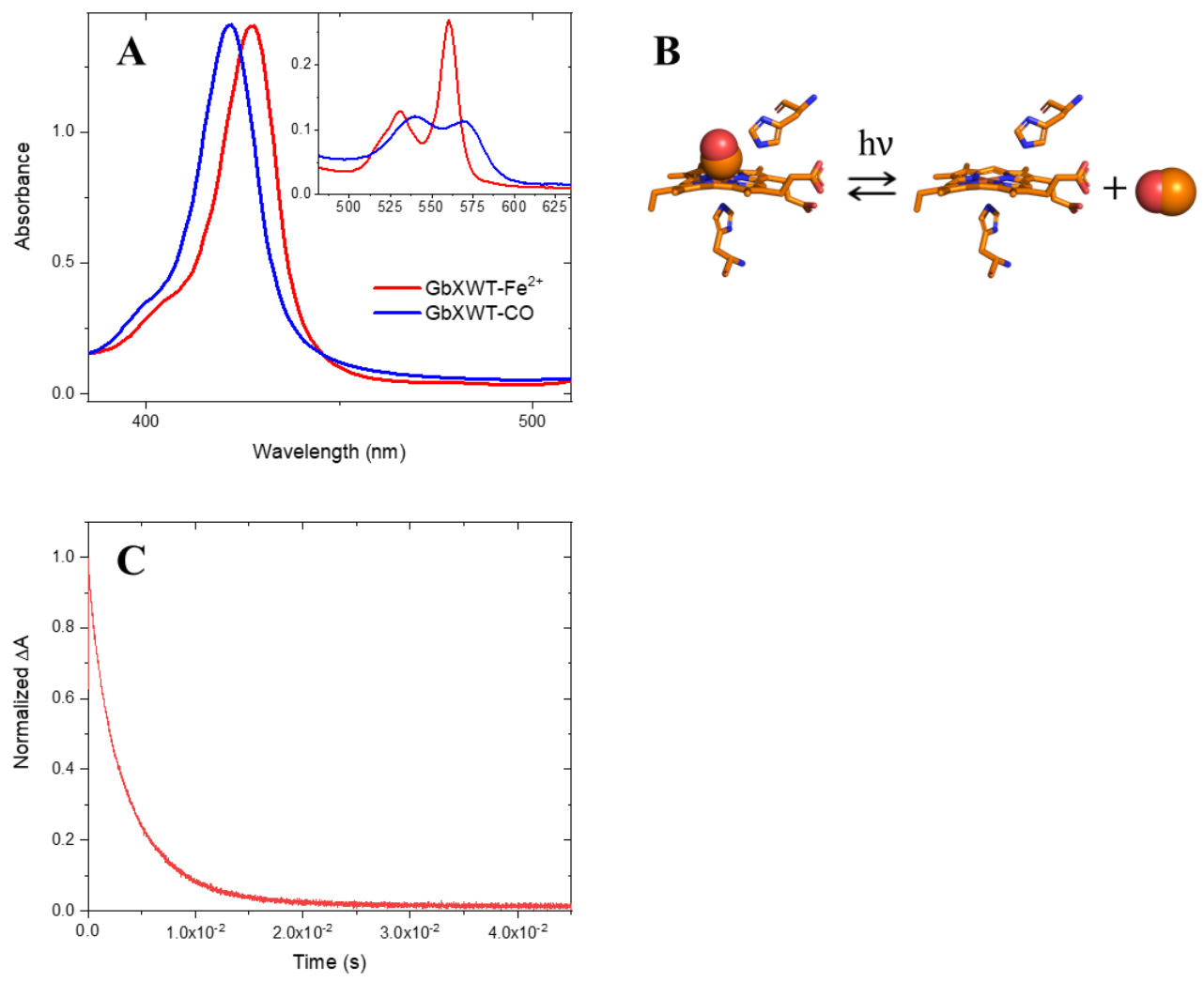

Figure 3.7. In transient absorption spectroscopy, time profiles of absorption changes that are associated with ligand binding/dissociation from proteins are measured. (A) Absorption spectrum of deoxy- and CO-GbX in the UV and visible (inset) region. (B) Simplified reaction 
diagram of $\mathrm{CO}$ photodissociation and rebinding to swMb. (C) Time resolved absorption trance for $\mathrm{CO}$ recombination to $\mathrm{GbX}$ on microsecond to millisecond timescale.

In general, a stopped-flow instrument is used for a rapid mixing of the ligand and its receptor and the rate of the ligand-receptor complex formation is probed by monitoring the change in absorption at certain wavelength. However, the application of stop flow is limited by its time resolution of $1 \mathrm{~ms}$ that does not allow for monitoring reactions on sub-millisecond timescale.

Transient absorption spectroscopy (TA), also named flash photolysis or pumpprobe method, is a method that uses a short pulse from a flash lamp or laser to phototrigger a chemical reaction and monitor the absorption changes of the entire process. This concept was first reported by Gibson in 1956 in study the CO interaction with myoglobin (Gibson, 1956). Compare to the conventional stopped-flow technique, the transient absorption spectroscopy can detect events that occurs on sub-millisecond time-scales and using novel femtosecond lasers, biological event such as bond cleavage and formation as well as structural relaxation can be monitored on the time-scales from picoseconds to seconds (Weinacht \& Pearson, 2018). Transient absorption spectroscopy is widely used to study photochemistry and biologically relevant photochemical process. However, the prerequisite of using TA is the present of a chromophore and photo-initiation of the reaction of interest.

Fortunately, the heme prosthetic group that absorbs across the visible spectrum and photo-liability of the iron ligand bond makes globins specifically attractive as a model system for study ligand binding reactions (Nienhaus, 2010). 


\subsubsection{TA set up}

The set-up of the home-built transient absorption spectroscopy instrument in our lab is illustrated in figure 3.8. The sample was in the $0.5 \times 1 \mathrm{~cm}$ quartz cuvette that was placed in a temperature-controlled cell holder (Flash 300, Quantum Northwest). The ligand photo-dissociation was triggered using a $532 \mathrm{~nm}$ output of Nd:YAG laser ( $5 \mathrm{~ns}$ pulse width, $1 \mathrm{~Hz}$ repetition Minilite II laser Continuum). The laser beam was directed into the center of the cuvette by use of two laser mirrors.

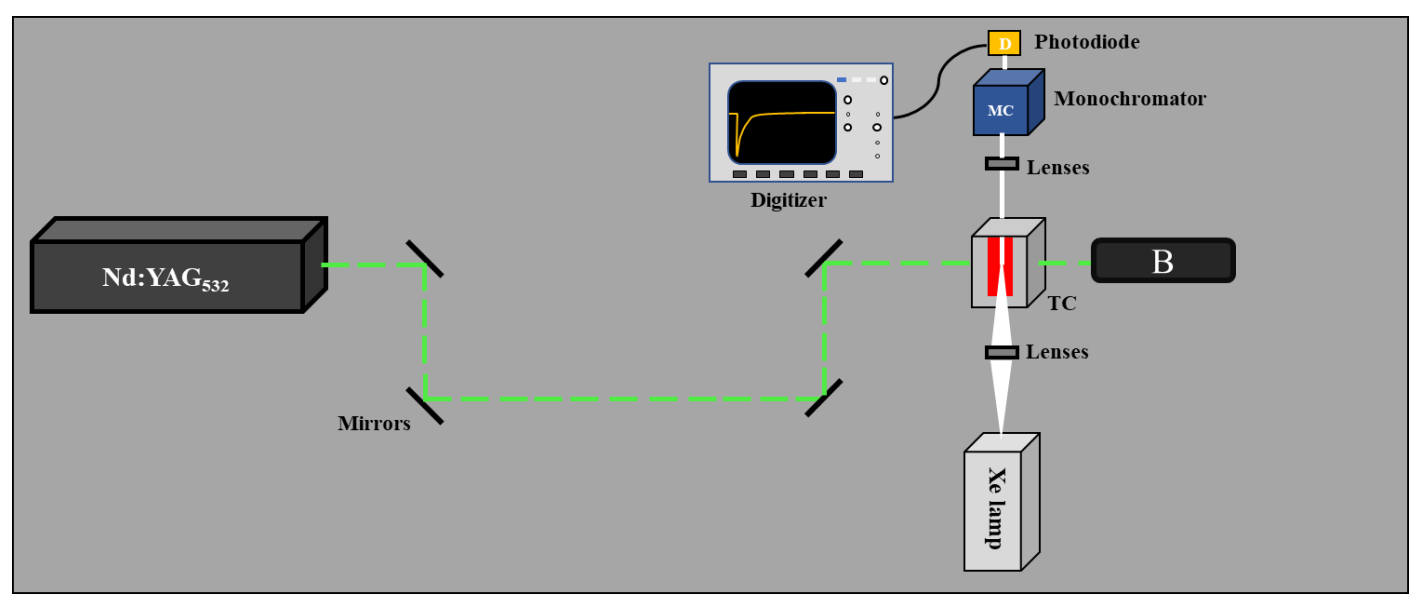

Figure 3.8. Top-view schematic of the transient absorption apparatus. TA components: the sample (red), temperature-controlled cuvette holder (TC), mirrors, pump beam (Nd:YAG532), beam blocker (B), lenses, probe beam (Xe lamp), monochromator (MC), photodiode (D) and Digitizer. Dashed line indicates pulsed light.

The $447 \mathrm{~nm}$ continuum wave probe beam (MDL-III-447, Changhun New Industries Optoelectronics Tech Co. Ltd) was focused on the center of the cuvette and emerging light was then focused on the entrance slit of a monochromator (Jobin Yvon) by focusing lenses. The change in absorbance was detected by a single photodiode (Model 818-BB-22, Newport) and the signal was digitized and stored in a $400 \mathrm{MHz}$ digitizer (Wave Surfer 42Xs, LeCroy). 


\subsubsection{TA sample preparation}

To prepare $\mathrm{CO}$ bound $\mathrm{Cygb}, \mathrm{Ngb}$ and $\mathrm{GbX}$ samples, the protein stock was first diluted by $50 \mathrm{mM}$ Tris- $\mathrm{HCl}$ buffer $(\mathrm{pH}$ 7.0) and gently mixed by fingertip then spin down by mini centrifuge to remove precipitate. The samples mixture was then transferred into $0.5 \mathrm{~cm} \times 1.0 \mathrm{~cm}$ or $0.2 \mathrm{~cm} \times 1.0 \mathrm{~cm}$ quartz cuvette by pipette and sealed with a septum cap. Argon was then employed to purge the sample for around $10 \mathrm{~min}$ to remove any $\mathrm{O}_{2}$ dissolved in the solution, $1 \mu \mathrm{l}$ of freshly prepared $\sim 1 \mathrm{mM}$ sodium dithionate was injected into the cuvette by Hamilton syringe. Next, the sample was purged by $\mathrm{CO}$ for around $9 \mathrm{~min}$ and the formation of the $\mathrm{CO}$ bound protein was verified by the UV-vis absorption spectroscopy (single-beam UV-vis spectrometer, Cary 50, Varian). Protein concentrations were calculated using the extinction coefficient at 532 $\mathrm{nm}$ of $10.7 \mathrm{mM}^{-1} \mathrm{~cm}^{-1}$ for the met form of the proteins

Ligand association kinetics.

Kinetics associated with $\mathrm{CO}$ rebinding to $\mathrm{GbX}$ were determined by fitting the absorption traces detected at $447 \mathrm{~nm}$ using a multi-exponential decays model (Equation $3.4)$.

$$
\Delta A=\sum_{i} A_{i} e^{\frac{t}{\tau_{i}}}
$$

Where $\tau_{\mathrm{i}}$ is the lifetime and $\mathrm{A}_{\mathrm{i}}$ are the amplitude for each step, respectively.

The fitting of the experimental data was performed by multi-exponential decay model in Origin (OriginLab Corp). The quality of the fit was assessed by assessing 
residuals for each fit and visual inspection Also, the error for the rate constants were calculated based on results from three independent experiments.

The maximum entropy method (MEM) was also applied to fitting the kinetic data The maximum entropy method is a mathematical inversion method, applied in diverse fields such as for example, neutron scattering, radio-astronomy, fluorescence as well as ligand interaction (Livesey \& Brochon, 1987; Steinbach, 1996).

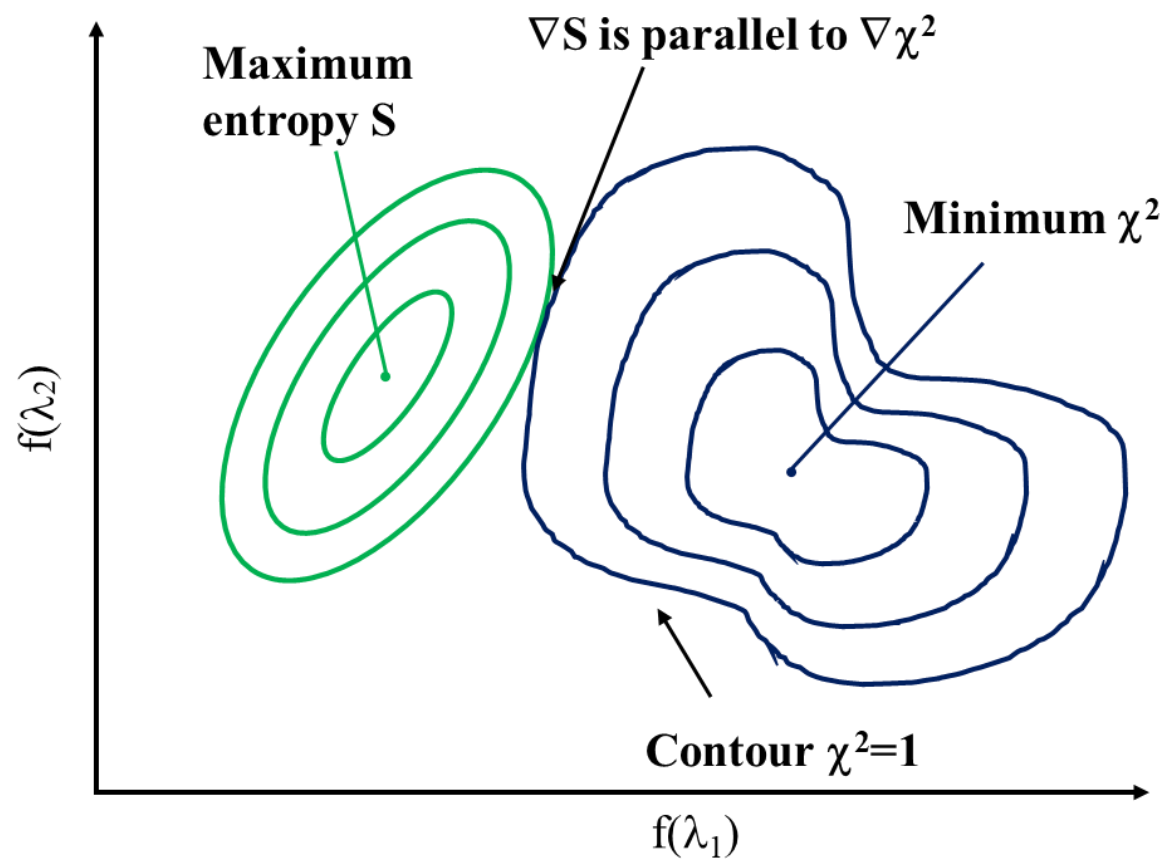

Figure 3.9. Contour plots of the statistic parameter $\chi^{2}$ and the entropy $S$ for a two dimensional $\mathrm{f}(\lambda)$. The maximum entropy solution equivalent to the point where the gradient of $\chi^{2}$ is parallel to the gradient of $\mathrm{S}$, where $\chi^{2}$ is close to 1 . Modified from Steinbach et al. (Steinbach et al., 1992).

The general principle of maximum entropy method is that a rate of distribution $f(\lambda)$ is displayed by a discrete set of data, $f\left(\lambda_{j}\right)$, and Shannon-Jaynes entropy, $S$, is employed to measure the uncertainty arise from the defining of the function $f\left(\lambda_{j}\right)$. The entropy $\mathrm{S}$ is maximized subject to constraining the statistic parameter $\chi^{2}$ to 1 (Figure 3.9). Consequently, the maximum entropy method does not bring any associations into 
$f(\lambda)$ nor establishes a particular model. Therefore, the experimental data directly present the rate distribution (Peter J. Steinbach et al., 2002; P.J. Steinbach et al., 1992).

The MemExp program designed by Steinbach et al.(Peter J. Steinbach et al., 2002) was applied in this study to analyze the kinetics data collected for $\mathrm{CO}$ rebinding to $\mathrm{GbX}$. The MemExp program combine the maximum entropy method (MEM) and either maxium likelihood (ML) or nonlinear least squares (NLS) fitting to analyze a general time-dependent data in terms of discrete and distributed lifetimes (Peter J. Steinbach, 2012; Peter J. Steinbach et al., 2002). One or two distributions of efective log-lifetimes, $\mathrm{g}(\log \tau)$ and $\mathrm{h}(\log \tau)$, are employed in the program to analyze kinetic data. According to equation 3.5 .

$$
F_{i}=D_{0} \int_{-\infty}^{+\infty} d \log \tau[g(\log \tau)-h(\log \tau)] e^{-t_{i} / \tau}+\sum_{k=0}^{3}\left(b_{k}-c_{k}\right)\left(\frac{t_{i}}{t_{\max }}\right)^{k}
$$

Where $\mathrm{D}_{0}$ is a normalization constant, the functions $\mathrm{g}(\log \tau)$ correspond to the distributions describing decaying kinetics and $\mathrm{h}(\log \tau)$ correspond to the distributions describing rising kinetics. And the polynomial function represents the experimental baseline. In order to maintain the baseline comparable in magnitude, the coefficients of them are scaled by the constant parameter $t_{\max }$. The experimental data can be used to estimate the constant $\mathrm{D}_{0}$, presuming that all kinetic processes involved in the temporal window of the measurements.

\section{Pseudo-first order reactions.}

Rebinding experiments were conduct in conditions of excess ligand concentrations, which generate pseudo-first order kinetics (Klostermeier \& Rudolph, 
2018). For ligand binding to pentacoordinate protein such as $\mathrm{Mb}$, a reaction describing formation of Protein:CO complex can be written as

$$
P+C O \underset{k_{H}}{\stackrel{k_{-H}}{\rightleftharpoons}} P: C O
$$

The rate law for $\mathrm{P}$ (protein) is

$$
\frac{d[P]}{d_{t}}=-k_{1}[P][C O]+k_{-1}[P: C O]
$$

The rate laws in terms of formation of $\mathrm{P}: \mathrm{CO}$ as

$$
\frac{d[P: C O]}{d t}=k_{1}[P][C O]-k_{-1}[P: C O]
$$

When the reaction performed under pseudo-first-order conditions, the concentration of $\mathrm{CO}$ will be constant over the reaction $\left([\mathrm{CO}] \approx \mathrm{CO}_{0}\right)$

$$
\frac{d[C O]}{d_{t}} \approx 0
$$

Simplify rate law for $\mathrm{P}$ and $\mathrm{P}: \mathrm{CO}$ to

$$
\frac{d[P]}{d_{t}}=-k_{1}[P] C O_{0}+k_{-1}[P: C O]
$$

and

$$
\frac{d[P: C O]}{d t}=k_{1}[P] C O_{0}-k_{-1}[P: C O]
$$

Based on mass conservation, we can express $[\mathrm{P}]$ as $\mathrm{P}_{0}-[\mathrm{P}: \mathrm{CO}]$ :

$$
\frac{d[P: C O]}{d t}=k_{1}\left(P_{0}-[P: C O]\right) C O_{0}-k_{-1}[P: C O]=k_{1} P_{0} C O_{0}+\left(-k_{1} C O_{0}-k_{-1}\right)[P: C O]
$$

and can separate the variables

$$
\frac{d[\mathrm{P}: \mathrm{CO}]}{k_{1} P_{0} C O_{0}-\left(-k_{1} C O_{0}+k_{-1}\right)[P: \mathrm{CO}]}=d t
$$

The integral on the left can be evaluated by substitution of $u$ for the denominator, with 


$$
\frac{d u}{d[P: C O]}=-\left(k_{1} C O_{0}+k_{-1}\right) \text { or } d[P: C O]=\frac{d u}{-\left(k_{1} C O_{0}+k_{-1}\right)}
$$

giving

$$
\frac{1}{-\left(k_{1} C O_{0}+k_{-1}\right)} \cdot \int \frac{1}{u} d u=1 \int_{0}^{t} d t
$$

and

$$
\frac{1}{-\left(k_{1} C O_{0}+k_{-1}\right)} \cdot \ln u=t
$$

Substituting back $\mathrm{u}$ and evaluating the integral from 0 to $[\mathrm{P}: \mathrm{CO}](\mathrm{t})$ then yields

$$
\frac{\ln \left(k_{1} P_{0} C O_{0}-\left(-k_{1} C O_{0}-k_{-1}\right)[P: C O]\right)-\ln \left(k_{1} P_{0} C O_{0}\right)}{-\left(-k_{1} C O_{0}-k_{-1}\right)}=t
$$

which can be solved for $[\mathrm{P}: \mathrm{CO}](\mathrm{t})$ :

$$
[P: C O](t)=P_{0} \frac{k_{1} C O_{0}}{k_{1} C O_{0}+k_{-1}}\left(1-e^{-\left(k_{1} C O_{0}+k_{-1}\right) t}\right)
$$

The exponential term in eq 3.18 has the form of a simple growth reaction with e observed rate constant

$$
k_{o b s}=k_{1} C O_{0}+k_{-1}
$$

Here, the $\mathrm{k}_{1}$ and $\mathrm{k}_{-1}$ is the $\mathrm{k}_{\mathrm{on}}$ and $\mathrm{k}_{\text {off }}$ of $\mathrm{CO}$ binding to protein, respectively. We can obtain the $\mathrm{k} 1$ and $\mathrm{k}-1$ by plot $\mathrm{k}_{\mathrm{obs}}$ (observed rate constant for binding at different concentrations $\mathrm{CO}_{0}$ ) as a function of $\mathrm{CO}_{0}$, since the dependence of $\mathrm{k}_{\mathrm{obs}}$ and concentration $\mathrm{CO}_{0}$ is linear, the slop is $\mathrm{k}_{1}$ and $\mathrm{y}$-axis intercept is $\mathrm{k}_{-1}$.

\section{Steady-state thermodynamics}

Time-resolved experiments performed as a function of temperature can be analyzed using, Arrhenius (Eq) and Eyring (Eq) equations to obtain thermodynamic parameters for ligand binding to heme proteins. Activation energy $\left(E_{a}\right)$ and preexponential factor (A) can be determined by using Arrhenius equation (Eq. 3.20) 


$$
\ln k=\ln A-\frac{E_{a}}{R T}
$$

where $\mathrm{A}$ is pre-exponential factor, $\mathrm{k}$ is rate constant, $\mathrm{R}$ is gas constant, $\mathrm{T}$ is absolute temperature in Kelvin. On the other side, activation enthalpy $\left(\Delta^{\ddagger} \mathrm{H}\right)$ and entropy $\left(\Delta^{\ddagger} \mathrm{S}\right)$ can be determined by Eyring equation:

$$
\ln \frac{k}{T}=-\frac{\Delta^{\ddagger} H}{R} \cdot \frac{1}{T}+\ln \frac{k_{B}}{h}+\frac{\Delta^{\ddagger} S}{R}
$$

where $\mathrm{k}_{\mathrm{B}}$ is Boltzmann constant, $\mathrm{h}$ is Planck's constant, $\mathrm{k}$ is rate constant, $\mathrm{R}$ is gas constant, and $\mathrm{T}$ is absolute temperature. It should be pay attention that $\mathrm{E}_{\mathrm{a}}$ and $\Delta^{ \pm} \mathrm{H}$ are related quantities and $\mathrm{E}_{\mathrm{a}}=\Delta^{\ddagger} \mathrm{H}+\mathrm{RT}$ for reaction in the solution (figure 3.10)(Atkins \& Paula, 2010).

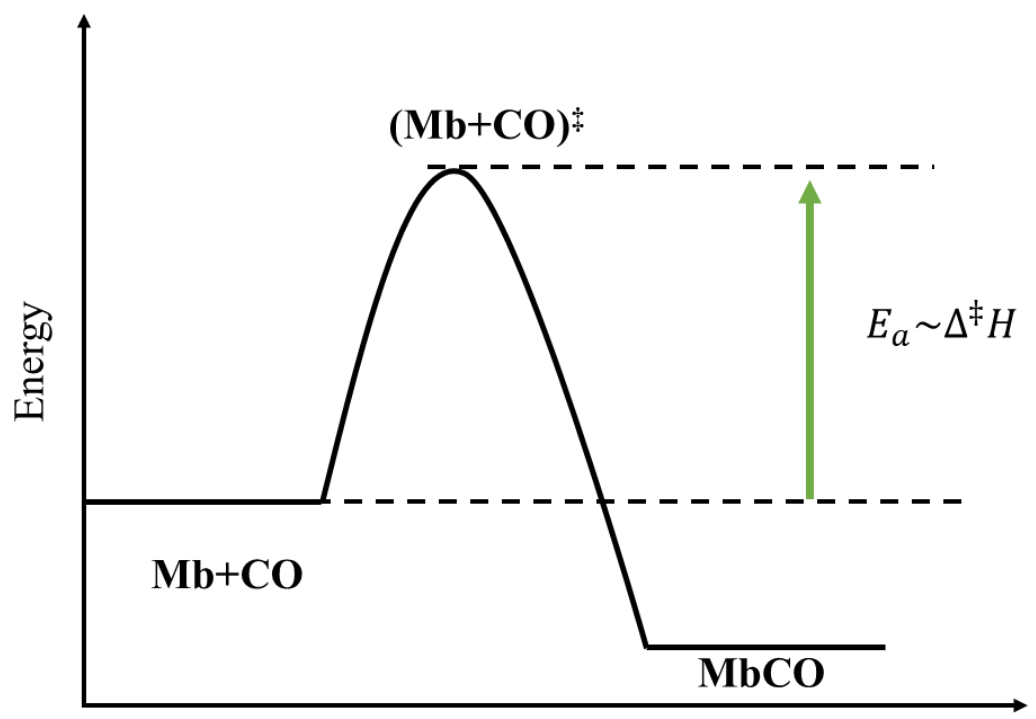

Reaction coordinate

Figure 3.10. An energy diagram for $\mathrm{CO}$ binding to $\mathrm{Mb}$.

\subsubsection{Photoacoustic calorimetry (PAC)}

\subsubsection{Introduction}

The changes in the electronic spectrum of chromophores can be explored by timeresolved optical methods with high sensitivity in the time range from picosecond to 
millisecond and these data provide information about local structure dynamics. The ability to probe directly the thermodynamic events occurring on these time scales in the proteins, in term of molar volumes and enthalpy changes is still limited (Larsen \& Mikšovská, 2007; Vetromile et al., 2011).

Photoacoustic calorimetry (PAC) which is one of the photothermal methods, is a useful techniques that capable to obtain the time scale of structural changes as well as to quantify structural changes in term of reaction volume and enthalpy changes occurring on physiologically relevant time scales in proteins (Gensch \& Viappiani, 2003; Larsen \& Mikšovská, 2007). Without a need for a chromophore or fluorophore, PAC is capable to observe the entire reaction enthalpy and volume change correlated with global structural changes in proteins in both magnitude and time ( $\sim 50 \mathrm{~ns}$ to $10 \mu \mathrm{s})$ (Mikšovská et al., 2008). PAC is widely used in study conformational changes associated with ligand photo-release from heme proteins, such as $\mathrm{CO}-\mathrm{Mb}$ or $\mathrm{CO}-\mathrm{Hb}$.

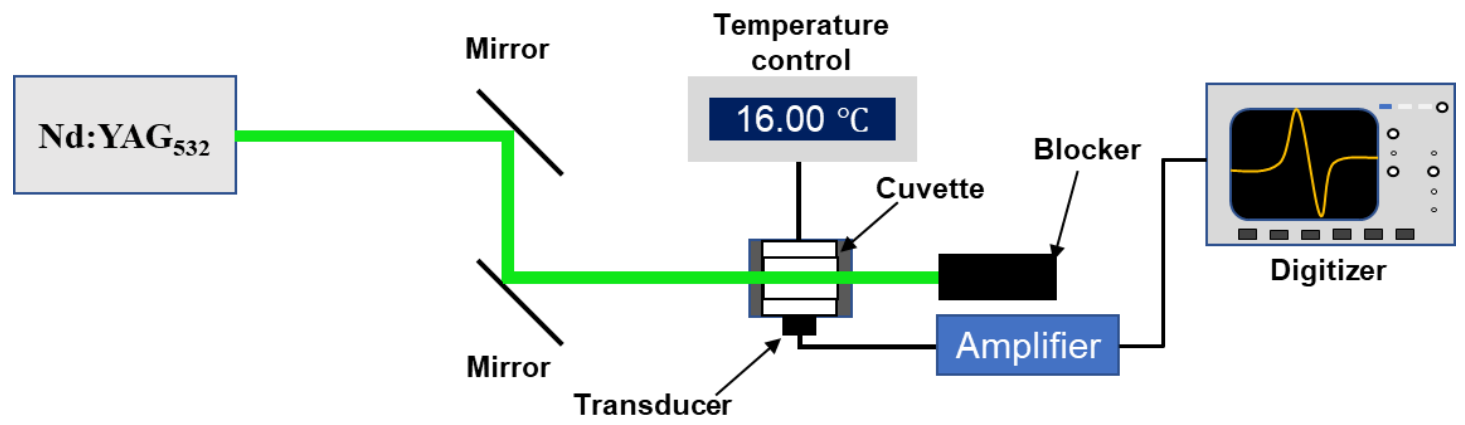

Figure 3.11. Schematic diagram of a home-build PAC instrument setup (top view).

The general physical principle behind the photoacoustic calorimetry is that upon photo-excitation of a molecule there is a accompanied dissipation of excess energy through vibrational relaxation to the ground state and concomitant thermal heating of 
the surrounding solvent (Gensch \& Viappiani, 2003; Larsen \& Mikšovská, 2007). In this thesis, PAC was employed to characterize energetic cost of structural changes upon ligand dissociation from $\mathrm{CO}$-bound and $\mathrm{O}_{2}$-bound $\mathrm{GbX}$ variants.

\subsubsection{PAC set up}

The home-built PAC instrument was described before (Mikšovská et al., 2008). A schematic representation of the PAC instrumental setup is shown in Figure 3.11. The $\mathrm{CO}$ bound GbX samples were placed into a $0.5 \times 1.0 \mathrm{~cm}$ quartz optical cuvette sealed by a septum cap. The cuvette was placed into a temperature-controlled cuvette holder (Flash 300, Quantum Northwest). The photo-dissociation of CO from GbX was triggered by a $5 \mathrm{~ns}$ laser pulse $(532 \mathrm{~nm}, 1 \mathrm{~Hz}$ repetition rate, Minilite II, Continuum) and the acoustic waves were detected by a piezoelectric detector attached by a thin layer of honey to the side of the cuvette. A schematic diagram of the wave propagation in the cuvette in PAC experiment is shown in Figure 3.12.

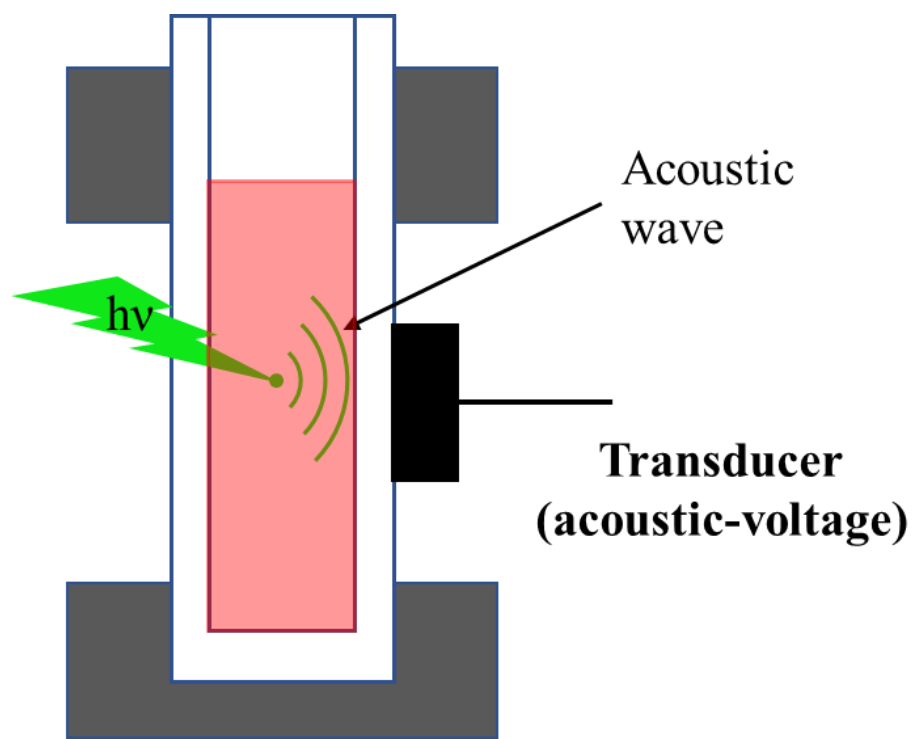

Figure 3.12. Schematic diagram of wave propagation from the sample to the detector in PAC measurements. 
The signal was amplified by a pre-amplifier (C6438-01, Hamamatsu) and recorded using a $400 \mathrm{MHz}$ digitizer (Wave Surfer 42Xs, LeCroy). Sample and reference (Fe(III)tetrakis(4-sulfonatophenyl)porphine, Fe(III)4SP, Frontier Scientifica Inc) traces were measured in the temperature range between 16 and $30^{\circ} \mathrm{C}$ under identical conditions. In order to improve the signal-to-noise ratio, 25-50 traces were averaged for each photoacoustic trace. Photoacoustic traces were then analyzed using Origin as described below.

\subsubsection{Quantum yield determination}

The quantum yield $(\Phi)$ for the biomolecular $\mathrm{CO}$ and $\mathrm{O}_{2}$ dissociation was determined as described previously (Belogortseva et al., 2007). All transient absorption measurements were carried out using $20 \mu \mathrm{M}$ protein samples in $50 \mathrm{mM}$ TrisHCl buffer, $\mathrm{pH}$ 7.0. Samples were transferred into a $5 \mathrm{~mm}$ path quartz cuvette and placed into a temperature-controlled holder (Quantum Northwest). Ligand photo-dissociation was triggered using a $532 \mathrm{~nm}$ output from a Nd:YAG laser (Minilite II, Contiuum). The probe beam (MDL-III-447, Changhun New Industries Optoelectronics Tech Co. Ltd) was propagated through the center of the cuvette and then focused on the input of a monochromator (Yvon-Jovin) by lenses. The intensity of the probe beam was detected by an amplified photodiode (Model 818-BB-22, Newport) and subsequently digitized (Wave Surfer 42Xs, $400 \mathrm{MHz}$ ). The quantum yield was determined by comparing the change in the sample absorbance at $447 \mathrm{~nm}$ with that of the reference. $\mathrm{CO}$ bound $\mathrm{Mb}$ was used as a reference to determine quantum yields as it has a high quantum yield of 
0.98 and the quantum yield is temperature independent (Schuresko \& Webb, 1978). Based on the absorption change of sample $\left(\Delta \mathrm{A}_{\text {sam }}\right)$ and reference $\left(\Delta \mathrm{A}_{\text {ref }}\right)$, the quantum yield was determined according to Eq 3.22:

$$
\Phi=\frac{\Delta A_{\text {sam }} \Delta \varepsilon_{r e f} \Phi_{r e f}}{\Delta A_{r e f} \Delta \varepsilon_{\text {sam }}}
$$

Where $\Delta \mathrm{A}_{\text {sam }}$ and $\Delta \mathrm{A}_{\text {ref }}$ are the change of the absorbance of sample and reference at 447 $\mathrm{nm}$, respectively, and $\Delta \varepsilon_{\text {sam }}$ and $\Delta \varepsilon_{\text {ref }}$ are the difference of the extinction coefficient between the $\mathrm{CO}$ or $\mathrm{O}_{2}$ bound and reduced form of the sample and the reference, respectively.

\subsubsection{PAC data analysis}

The data analysis for PAC has been previously reviewed (Gensch \& Viappiani, 2003; Larsen \& Mikšovská, 2007). The amplitude of the sample acoustic signals is the difference between the first maximum and minimum of the sample acoustic wave as show in figure 3.13 , this is also applied for reference. The following equation describe the relation between the amplitude of the sample acoustic wave and heat released to the solvent, $\mathrm{Q}$, and nonthermal volume change $\left(\Delta \mathrm{V}_{\text {nonthermal }}\right)$ :

$$
S=K E_{a}\left(Q \frac{\beta}{C_{p} \rho}+\Delta V_{\text {nonthermal }}\right)
$$

The $\mathrm{Q}\left[\beta /\left(\mathrm{C}_{\mathrm{p}} \rho\right)\right]$ term corresponds to the thermal volume change $\left(\Delta \mathrm{V}_{\text {thermal }}\right)$. The photo-triggered volume changes such as conformation rearrangement, cleavage of the Fe- $\mathrm{O}_{2}$ bond are described as $\Delta \mathrm{V}_{\text {nonthermal. }} \beta$ is the thermal expansion coefficient and it is temperature dependent for water. The term $\mathrm{E}_{\mathrm{a}}$ is the number Einsteins absorbed and $K$ is the instrument response parameter. A signal for calorimetric reference was measured 
under conditions identical to that for sample in order to eliminate the instrument response parameter and calibrate the instrument. Fe(III)4SP was used as a reference in PAC studies as it does not undergo any photochemistry and the molecule releases absorbed energy into the surrounding solvent with a yield of unity (Abbruzzetti et al., 1999).

The amplitude of acoustic signal for reference $\mathrm{R}$ can be expressed as:

$$
R=K E_{a} E_{h \nu}\left(\frac{\beta}{c_{p} \rho}\right)
$$

Where $E_{h v}$ is the energy of the photon at the excitation wavelength.

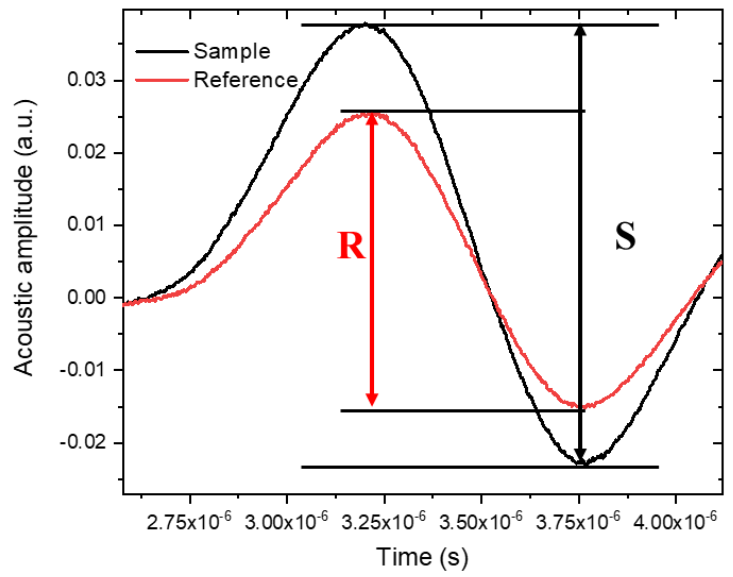

Figure 3.13. Illustrative PAC acoustic traces for sample and reference compound.

The acoustic traces were collected as a function of temperature to separate the nonthermal and thermal volume changes.

According to equation 3.25 , by plot the ratio of the sample and reference acoustic waves amplitude as a function of the temperature dependent term $\left[\beta /\left(\mathrm{C}_{\mathrm{p}} \rho\right)\right]$, the nonthermal volume change can be obtained from the slope and the amount of heat released to the solution can be extract from the intercept of the linear plot. 


$$
\emptyset E_{h v}=\left(\frac{S}{R}\right) E_{h v}=Q+\left(\frac{C_{p} \rho}{\beta}\right) \Delta V_{\text {nonthermal }}
$$

For processes with the quantum yield $(\Phi)$ less than 1 , the reaction enthalpy $(\Delta H)$ and volume change $(\Delta V)$ can be determined according to equation 3.26 and 3.27 , respectively.

$$
\begin{aligned}
& \Delta H=\frac{E_{h v}-Q}{\Phi} \\
& \Delta V=\frac{\Delta V_{\text {nonthermal }}}{\Phi}
\end{aligned}
$$

Because the piezoelectric transducer is capable to detect not only the amplitude of the acoustic wave but also the temporal profile of the acoustic wave, reactions occurring within the time resolution $(\sim 50 \mathrm{~ns}$ to $10 \mu \mathrm{s})$ of the PAC instrumentation can be characterized in term of their time constant and activation enthalpy and entropy change. The presence of a kinetic event occurring between $50 \mathrm{~ns}$ and $10 \mu \mathrm{s}$ is evident from the shift of the sample acoustic wave to lower frequencies with respect to the reference wave (figure 3.14).

The function describing the sample acoustic wave $\mathrm{E}(\mathrm{t})$ is the convolution of a timedependent heat source $(\mathrm{H}(\mathrm{t}))$ with the instrument response function $\mathrm{T}(\mathrm{t})$ according to Eq 3.28.

$$
E(t)=H(t) \otimes T(t)
$$

For a two-step process, the time dependence of the concentration for A and B can be expressed using equation 3.30 and 3.31 .

$$
\begin{aligned}
& A \stackrel{k_{1}}{\rightarrow} B \stackrel{2_{2}}{\rightarrow} C \\
& {[A]=A_{0} e^{\frac{t}{\tau_{1}}}}
\end{aligned}
$$




$$
[B]=\frac{A_{0} k_{1}}{k_{2}-k_{1}}\left(e^{-\frac{t}{\tau_{1}}}-e^{-\frac{t}{\tau_{1}}}\right)
$$

The time-dependent heat source can be expressed by equation 3.31 (Schaberle et al., 2010).

$$
H(t)=\emptyset_{1} e^{-\frac{t}{\tau_{1}}}+\frac{\emptyset_{2} k_{1}}{k_{2}-k_{1}}\left(e^{-\frac{t}{\tau_{1}}}-e^{-\frac{t}{\tau_{2}}}\right)
$$

Deconvolution the sample acoustic trace provides $\phi_{1}$ and $\phi_{2}$ parameters that correspond to the ratio of the amplitude for the sample and reference acoustic wave for the first and second step of the reaction, respectively, as well relaxation time $\tau_{1}$ and $\tau_{2}$. The software SoundAnalysis (Quantum Northwest) was used to analyze the PAC data. The $\tau_{1}$ was fixed as $1 \mathrm{~ns}$, assuming the first kinetic process occurs within the laser pulse and the remaining three parameters $\left(\tau_{2}, \phi_{1}\right.$ and $\left.\phi_{2}\right)$ were varied. The time-dependent heat source function, $\mathrm{H}(\mathrm{t})$, is calculated by estimating the $\phi_{1} \phi_{2}$ and $\tau_{2}$ parameters, and then convoluted with $\mathrm{T}(\mathrm{t})$ function that corresponds to the acoustic wave for the calibration compound. The calculated acoustic wave for the sample, $\mathrm{E}_{\text {cal }}(\mathrm{t})$, is is then compared to the measured acoustic wave for the sample and fitting parameters $\phi_{1} \phi_{2}$ and $\tau_{2}$ until a satisfactory fit is obtained. The quality of the fit is evaluated by visual inspection of the residuals. For processes that occur with a quantum yield, $\Phi$, that is temperature dependent in the temperature range used in PAC measurements, the thermodynamic parameters for the fast phase $(\tau<50 \mathrm{~ns})$ are determined by plotting $\left[\mathrm{E}_{\mathrm{hv}}(\phi-1) / \Phi\right]$ versus $\left(\mathrm{C}_{\mathrm{p}} \rho / \beta\right)$ according to Eq. 3.41 and the volume and enthalpy changes for the subsequent steps are obtained by plotting $\left(\mathrm{E}_{\mathrm{h} v} \phi / \Phi\right)$ versus $\left(\mathrm{C}_{\mathrm{p}} \rho / \beta\right)$ according to Eq 3.34. (Peters et al., 1992). 


$$
\begin{aligned}
& \frac{E_{h v}(\phi-1)}{\Phi}=-\Delta H+\left(\frac{C_{p} \rho}{\beta}\right) \Delta V \\
& \frac{\phi E_{h v}}{\Phi}=-\Delta H+\left(\frac{C_{p} \rho}{\beta}\right) \Delta
\end{aligned}
$$

Where $\Delta \mathrm{H}$ and $\Delta \mathrm{V}$ correspond to the reaction enthalpy and volume change, respectively.

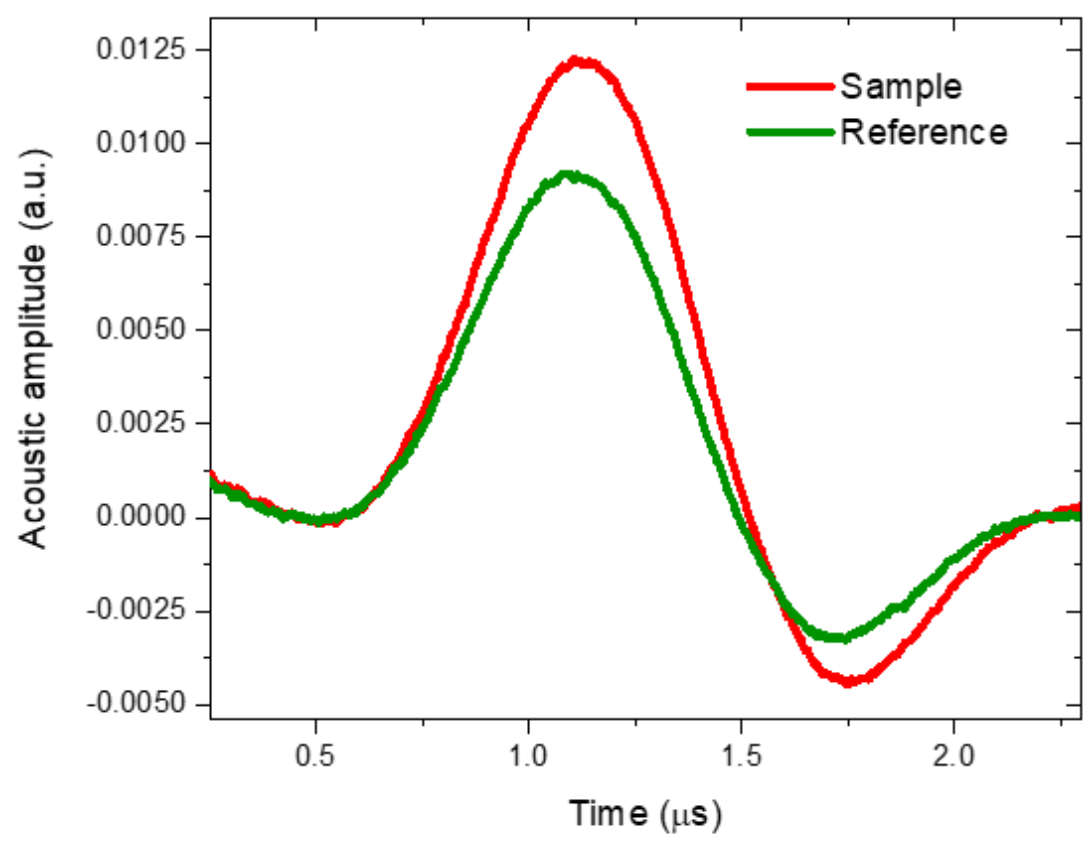

Figure 3.14. Illustrative PAC acoustic traces for the sample acoustic trace is shifted in phase compare to the reference trace.

\subsubsection{Stability studies}

The acid denaturation of met form or $\mathrm{O}_{2}$ bound $\mathrm{GbX}$ variants was probed by observing the decrease in absorbance of the Soret band proteins upon acidification of protein samples. Aliquots $(3-55 \mu \mathrm{L})$ of $2 \mathrm{M}$ or $4 \mathrm{M} \mathrm{HCl}$ were added to $2 \mathrm{~mL}$ of $10 \mu \mathrm{M}$ or $8 \mu \mathrm{M}$ protein in $5 \mathrm{mM}$ citrate/borate/phosphate buffer ( $\mathrm{pH} 8.0$ ), containing $0.1 \mathrm{M}$ $\mathrm{NaCl}$. After 10 min of equilibration at room temperature, $\mathrm{UV}$-vis spectra and $\mathrm{pH}$ of the solution were recorded. The UV-vis spectra were then corrected for a dilution and the 
fraction of unfolded protein was determined using absorbance values at $415 \mathrm{~nm}$ according to equation 3.35 .

$$
f_{\text {unfold }}=\frac{A_{0}-A}{A_{0}-A_{T}}
$$

where $\mathrm{A}_{0}$ is the protein absorbance at neutral $\mathrm{pH}, \mathrm{A}$ is the absorbance at $415 \mathrm{~nm}$ at any $\mathrm{pH}$ and $\mathrm{A}_{\mathrm{T}}$ is the absorbance at $415 \mathrm{~nm}$ at lowest $\mathrm{pH}$ value measured. The values of the fraction of unfolded protein, $f_{\text {unfolded, were plotted as a function of } \mathrm{pH} \text { and }}$ analyzed using equation 3.36 :

$$
f_{\text {unfold }}=\frac{\alpha_{N}+m_{N} \times p H+\left(\alpha_{D}+m_{D} \times p H\right) \times 10^{\Delta V_{H^{+}} \times\left(p H_{\text {mid }}-p H\right)}}{1+10^{\Delta V_{H^{+}} \times\left(p H_{m i d}-p H\right)}}
$$

Where $\mathrm{pH}_{\text {mid }}$ is the midpoints of the unfolding transitions and $\Delta \mathrm{V}_{\mathrm{H}}{ }^{+}$is used to describe the protons bound or released during the transition (Karp et al., 2007).

\subsubsection{Cyanide affinity test}

The affinity constants for cyanide binding to WT GbX and mutants were determined by monitoring the changes in the protein absorption spectrum upon addition of sodium cyanide. The titrations were performed using $8 \mu \mathrm{M}$ protein in $50 \mathrm{mM}$ Tris buffer $\mathrm{pH} 7.0$ at room temperature. The titrating solution was $750 \mathrm{mM} \mathrm{NaCN}$ solubilized in the same buffer. The $\mathrm{NaCN}$ concentration in protein samples was varied between $0.5 \mathrm{mM}$ and $150 \mathrm{mM}$. The spectrum was obtained after 2 min stirring by microstir bar after each $\mathrm{NaCN}$ addition to achieve equilibrium. The UV-vis spectra were corrected for dilution and scattering using FluorTools (a|e, FluorTools.com). 


\subsection{Methods: Chapter 2}

\subsubsection{Preparation of ZnPPIX reconstituted hexacoordinate globin}

Heme was extracted based on previously published 2-butanone methods (Teale, 1959). Wild type Cygb, Ngb and mutants are denatured by addition of concentrated $\mathrm{HCl}$ until $\mathrm{pH}$ decreased to 2.0 to release the native heme group from the protein. The sample mixture was then transfer into a $25 \mathrm{~mL}$ volumetric flask by glass pipette and the identical volume of ice-cold 2-butanone was added (Acros Organics, $99+\%$ spectroscopic grade). The mixture was vigorously shaken and placed on ice for $\sim 2 \mathrm{~min}$ until the aqueous phase and organic phase were separated. The colorless aqueous phase containing the unfolded apo-protein was extracted and dialyzed overnight against 50 $\mathrm{mM}$ TrisHCl $\mathrm{pH} 2.5$ to remove residual 2-butanone.

Next day, zinc protoporphyrin IX (ZnPPIX, Frontier Scientific) solution was prepared by solubilizing ZnPPIX powder in $1 \mathrm{M}$ freshly prepared $\mathrm{NaOH}$. ZnPPIX solution was mixed with the apo-protein sample in 1:1 molar ratio and the solution $\mathrm{pH}$ was then adjusted to be above 10. The ZnPPIX protein mixture was then incubated on ice for $\sim 2$ hours and $\mathrm{GuHCl}$ was added to achieve a final concentration of $6 \mathrm{M}$. The sample mixture was then dialyzed against $50 \mathrm{mM}$ Tris- $\mathrm{HCl}, \mathrm{pH} 7.0$ to allow the ZnPPIX-reconstituted protein gradually to refold into native conformation. After dialysis, the ZnPPIX-reconstituted protein were filtered, and their absorption spectra were recorded to verify the ZnPPIX incorporation into the heme pocket and aliquot then store at $4^{\circ} \mathrm{C}$. 


\subsubsection{Fluorescence spectroscopy}

The definition of fluorescence is a molecular transition from the lowest vibrational level of the energy level $\mathrm{S}_{1}$ to any vibrational level of the ground state, $\mathrm{S}_{0}$, with a concomitant photon emission (figure 3.15) (Lakowicz, 2007). Molecules that are fluorescent are called fluorophores and are distinguished by their emission spectra maxima $\left(\lambda_{\max }\right)$, lifetime of the $\mathrm{S}_{1} \rightarrow \mathrm{S}_{0}$ transition $\left(\tau_{\mathrm{f}}\right)$ and fluorescence quantum yield $\left(\Phi_{\mathrm{F}}\right)$.

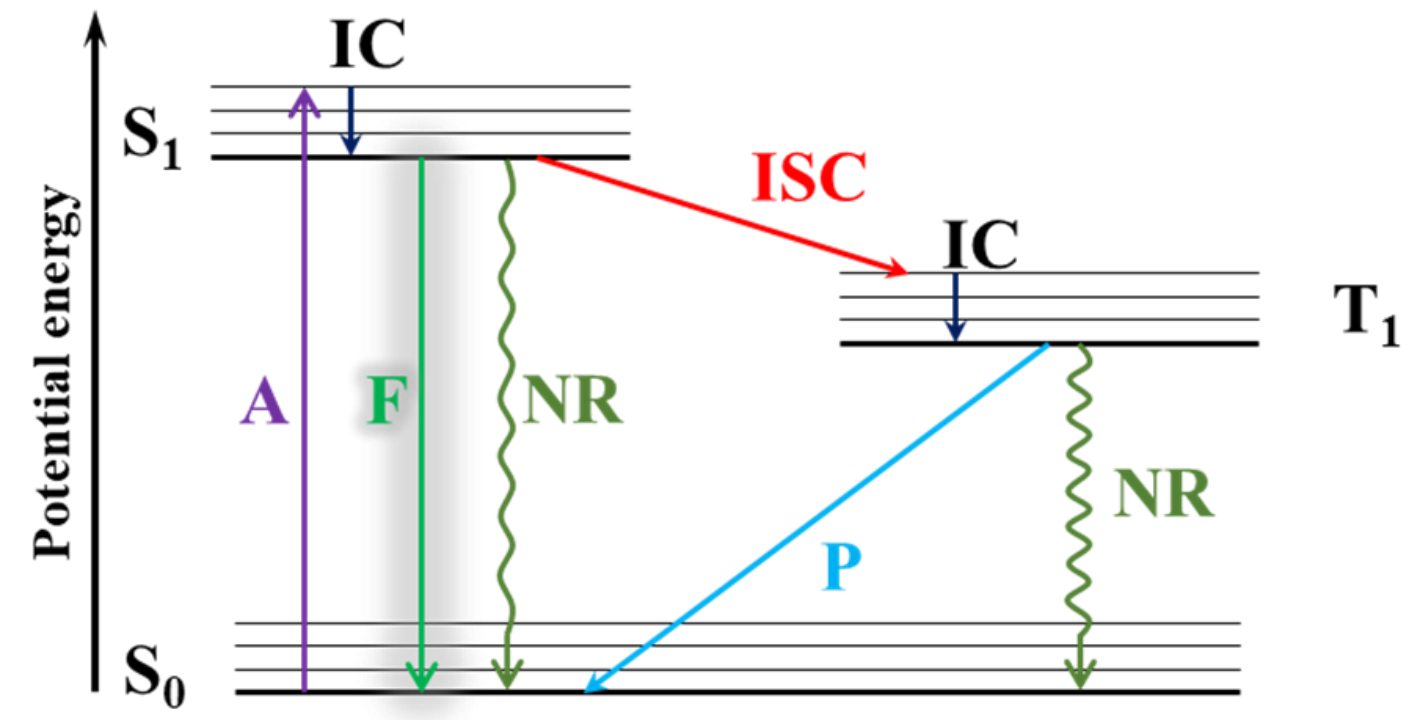

Figure 3.15. Simplified Jablonski diagram repersent electronic transitions between different electronic states. A as absorption, IC as internal conversion, ISC as intersystem crossing, F as fluorescence, $\mathrm{NR}$ as nonradiative decay, $\mathrm{P}$ as phosphorescence, $\mathrm{S}$ as singlet state, $\mathrm{T}$ as triplet state.

The fluorescence studies of proteins focus on characterization of either intrinsic or extrinsic fluorescence probes. Tryptophan, tyrosine, and phenylalanine residues are usual intrinsic probes in proteins while the extrinsic probes are organic fluorophores that are non-covalently or covalently bound to the protein , for example, porphyrins , 
8-anilino-1-naphthalenesulphonic acid (1,8-ANS), fluorescein (Ross \& Jameson, 2008). Fluorescence spectroscopy is extensively used in studies of protein conformation and structural dynamics due to its high sensitivity to conformational changes and structural fluctuations of the fluorophore surrounding (Lakowicz, 2007). In short, the distinct properties of fluorescence such as emission spectrum, lifetime, and quantum yield, can be altered by any excited-state event including conformational changes, drug binding, presence of a quencher etc (Valeur, 2001).

\subsubsection{Steady-state fluorescence spectroscopy}

Steady-state fluorescence emission spectra were recorded using Cary Eclipse Fluorescence Spectrophotometer. The excitation wavelength used for fluorescence studies of intrinsic Trp residues in Ngb, Cygb and mutants was at $295 \mathrm{~nm}$ and the spectra were recorded using a $10 \mathrm{~nm}$ monochromator slits whereas zinc protoporphyrin IX (ZnPPIX) reconstituted proteins were excited at $421 \mathrm{~nm}$ and $10 \mathrm{~nm}$ monochromator slits were used. The protein samples were prepared by diluting the protein from stock solution in $50 \mathrm{mM}$ Tris buffer, $\mathrm{pH} 7.0$, to a final concentration of $4 \mu \mathrm{M}$ and the protein samples were then transferred into a $0.2 \times 1.0 \mathrm{~cm}$ quartz cuvette by micropipette. Methyl viologen $\left(\mathrm{MV}^{2+}\right)$ quenching study were performed by titrating $4 \mu \mathrm{M} \mathrm{ZnPPIX}$ reconstituted protein with increasing concentration $\mathrm{MV}^{2+}$. The emission spectra from $540 \mathrm{~nm}$ to $740 \mathrm{~nm}$ were recorded as well as UV-vis spectra for inner filter correction. 


\subsubsection{Steady-state frequency-domain fluorescence lifetime}

The definition of fluorescence lifetime is the time a fluorophore spends in the excited state prior to the $\mathrm{S}_{1} \rightarrow \mathrm{S}_{0}$ transition (Lakowicz, 2007). Frequency-domain (FD) or phase-modulation measurements can determine the decay of the fluorophore from excited to the ground state (Holde et al., 1998). In FD measurements, the fluorophore is excited by a sinusoidaly modulated light. The time-delay of the fluorescence emission is governed by the modulation frequency of excitation ( $\omega=2 \pi$ frequency), which result in a phase shift $\left(\phi_{\omega}\right)$ to lower frequencies (figure 3.16).

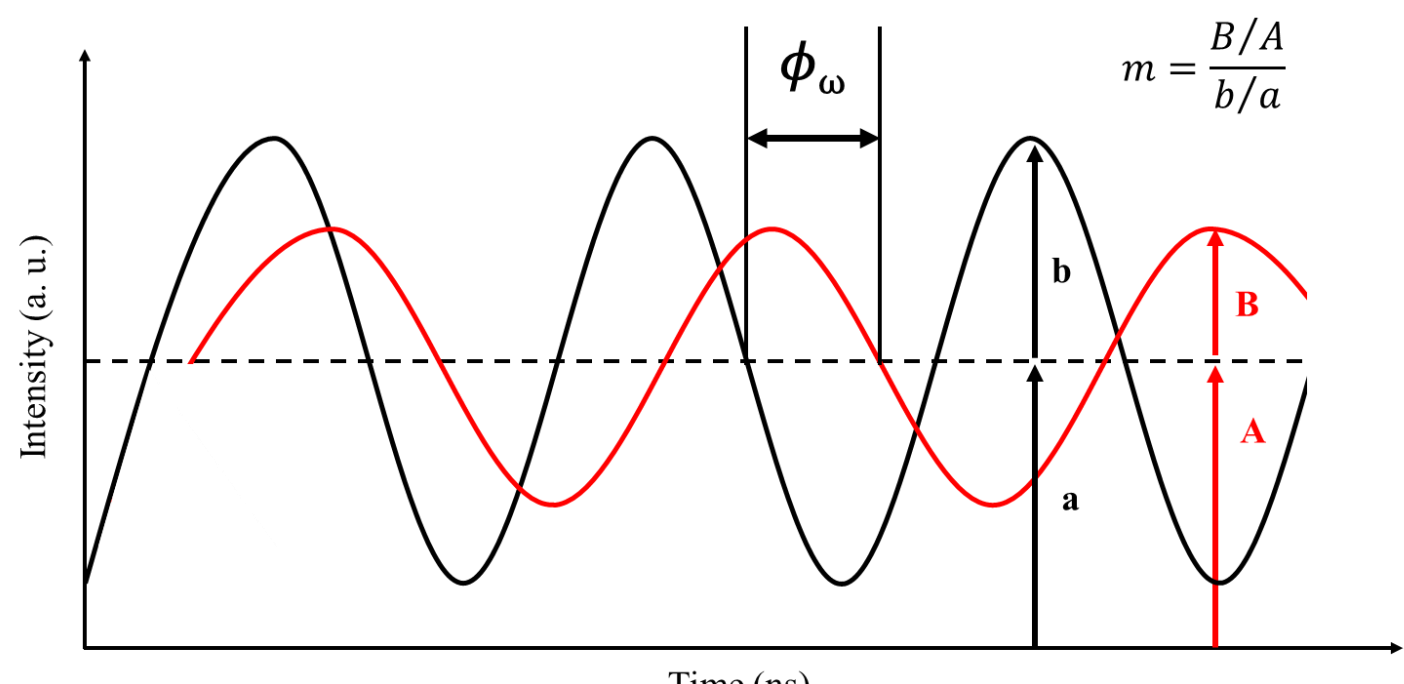

Figure 3.16. The modulation of emission (red) is decreased by modulation of excitation light intensity (black) and result in phase shift. The dash line indicated average intensity for both waves. The modulation ratio $(\mathrm{m})$ is determined by the amplitude of the average intensity (a, A) and the offset from the average intensity $(b, B)$ of emission and excitation.

For a single-exponential decay, phase lifetime $\left(\tau_{\phi_{\omega}}\right)$ and modulation $\left(\tau_{m_{\omega}}\right)$

lifetime can be determined if modulation frequency $(\omega)$ is known according Eq3.37 and 3.38:

$$
\tau_{\phi \omega}=\omega^{-1} \tan \phi
$$




$$
\tau_{m \omega}=\frac{1}{\omega}\left[\frac{1}{m^{2}}-1\right]^{1 / 2}
$$

where $\mathrm{m}$ is the modulation ratio and $\phi$ is the phase shift. The depopulation of the excited state of fluorophore is frequently a heterogeneous procedure, which need to use multi-exponential model rather than single exponential decay. Total intensity observed $I(t)$ is defined as sum of first-order processes

$$
I(t)=\sum \alpha_{i} e^{-t / \tau_{i}}
$$

where $\tau_{i}$ and $\alpha_{i}$ stand for fluorescence lifetimes and pre-exponential factors associated with each fluorescence decay procedure. Particularly, if the same fluorophore is found in different environments, the pre-exponential factors $\left(\alpha_{i}\right)$ values are proportional to the fractional population of fluorophore in each environment (Lakowicz, 2007). The fraction of fluorescence intensity of each component that contributes to the multi-exponential decay is related to the pre-exponential factors according to equation 3.40(Lakowicz et al., 1984).

$$
f_{i}=\frac{\alpha_{i} \tau_{i}}{\sum_{j} \alpha_{j} \tau_{j}}
$$

Transforms $N_{\omega}$ and $D_{\omega}$ can be obtained from multi-exponential decays of phase and modulation lifetimes, which are defined as

$$
\begin{aligned}
& N_{\omega}=\frac{\sum_{i} \frac{\alpha_{i} \omega \tau_{i}^{2}}{\left(1+\omega^{2} \tau_{i}^{2}\right)}}{\sum_{i} \alpha_{i} \tau_{i}} \\
& D_{\omega}=\frac{\sum_{i} \frac{\alpha_{i} \tau_{i}}{\left(1+\omega^{2} \tau_{i}^{2}\right)}}{\sum_{i} \alpha_{i} \tau_{i}}
\end{aligned}
$$

Then, equation 3.43 and 3.44 can be used to calculate the modulation ratio and phase angle. 


$$
\begin{aligned}
& m_{\omega, \text { calc }}=\left(N_{\omega}^{2}+D_{\omega}^{2}\right)^{1 / 2} \\
& \tan \phi_{\omega, \text { calc }}=\frac{N_{\omega}}{D_{\omega}}
\end{aligned}
$$

\subsubsection{Time-resolved fluorescence lifetime measurement}

Determination of excited-state fluorescence lifetime was performed on Chronos Spectrofluorometer (ISS) using $280 \mathrm{~nm}$ laser diode with $300 \mathrm{~nm}$ bandpass excitation filter (Andover Corp.) and a $500 \mathrm{~nm}$ or $550 \mathrm{~nm}$ long pass emission filter (Andover Corp.). ZnPPIX-proteins (in 50mM TrisHCl, $\mathrm{pH}$ 7.0) and reference (POPOP in ethanol, $\left.\tau_{\text {ref }}=1.35\right)$ were placed in $0.5 \times 1.0 \mathrm{~cm}$ quartz cuvette. The phase shift and modulation ratios were recorded on 15 modulation frequencies between 20-200 MHz and analyzed using Vinci Software (ISS).

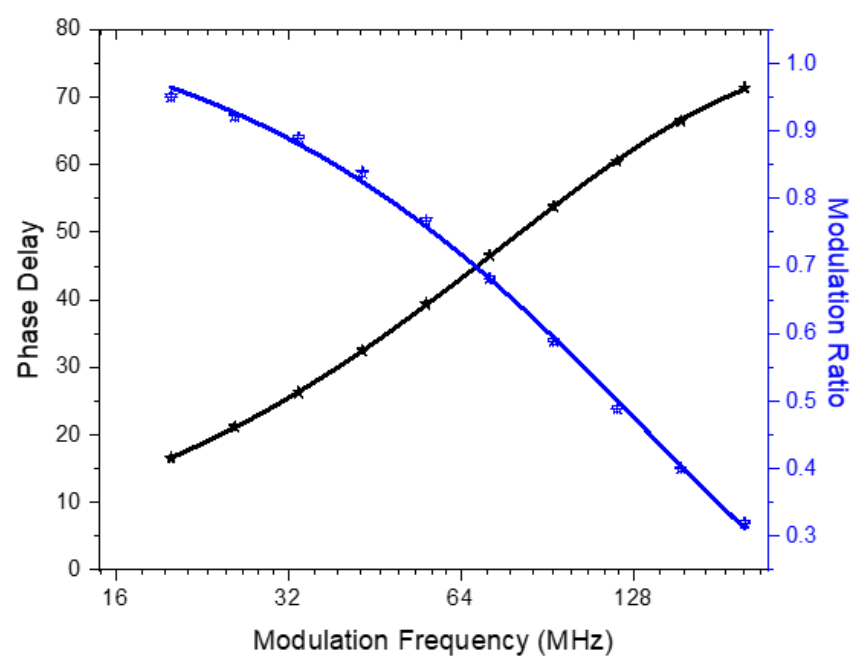

Figure 3.17. Phase delay $\left(\phi_{\omega}\right)$ and modulation ratio $(\mathrm{m})$ versus modulation frequency $(\omega)$ for POPOP reference compound.

\subsubsection{Phosphorescence}

Fluorophore molecules in the lowest vibrational level of the excitated state $\mathrm{S}_{1}$ can undergo an intersystem crossing to the excited triplet state $\left(\mathrm{T}_{1}\right)$. This nonradiative 
transition is caused by spin conversion which leads to the electron of excited state $\left(\mathrm{T}_{1}\right)$ and ground $\left(\mathrm{S}_{0}\right)$ with the same spin $(\uparrow \uparrow)$ (Lakowicz, 2007). The conversion from the triplet state to the ground state occurs on millisecond to second timescale and due to fact that the $T_{1} \rightarrow S_{0}$ transition is spin-forbidden and requires change in multiplicity (Lakowicz, 2007). Since $\mathrm{T}_{1}$ has lower energy than $\mathrm{S}_{1}$, phosphorescence spectra are red shifted compared to fluorescence (Lakowicz, 2007). In addition, because the quantum yield for phosphorescence are usually low $\left(\Phi_{P} \sim 10^{-6}\right)$, determination of the phosphorescence properties requires very concentrated samples and the measurements are carry out at low temperatures (Lakowicz, 2007). Also, as $\mathrm{O}_{2}$ is a very efficient quencher for phosphoresce, sample need to be thoroughly deoxygenated.

\subsubsection{Phosphorescence measurement}

Transient absorption spectroscopy was used to monitor phosphorescence decay of ZnPPIX-incorporated globins. ZnPPIX-reconstituted samples $(20 \mu \mathrm{M})$ were prepared in a $0.5 \times 1.0 \mathrm{~cm}$ quartz cuvette, sealed with a septum cap and parafilm. The sample was wrapped by foil to avoid light exposure and deoxygenated with argon for one hour while gently stirring using a micro stir bar. The cuvette containing sample was then placed into a cell holder and excited with a Nd:YAG laser (Minilite, Continuum) with a $532 \mathrm{~nm}$ output (pump beam). The absorbance of the triplet state was monitored using a $447 \mathrm{~nm}$ diode-pumped solid-state laser (MDL-III-447, Changehun New Industries Optoelectronics Tech Co. Ltd.). 


\section{IMPACT OF THE DISULFID BRIDGE AND DISTAL HISTIDINE ON}

\section{LIGAND MIGRATION IN GLOBIN X.}

\subsection{Introduction}

Globin is a sub-super family of heme proteins that carry out a variety of functions in different organisms such as oxygen storage and transport, catalysis, cellular signaling, and electron transfer (T Burmester \& Hankeln, 2014; Larsen \& Mikšovská, 2007). Hemoglobin $(\mathrm{Hb})$ and myoglobin $(\mathrm{Mb})$ are the two most well-studied globins, which were considered the only two globins in vertebrates. However, in the past two decades, sequences of new members of globin family where discorded in the Expressed Sequence Tags (EST) database including sequence for neuroglobin (Ngb), cytoglobin (Cygb), globin E and globin X (T Burmester \& Hankeln, 2014). Globin X (GbX) consists of $\sim 200$ amino acids and thus is larger than a typical globin ( $\sim 150$ amino acids), and it is mainly found in fish and amphibians but seemly lost in birds and mammals (Dröge \& Makałowski, 2011). Like Cygb, GbX has extended N-terminal and Cterminal, and it has been shown that myristylation and palmitoylation of residues located on the N-terminal extension of $\mathrm{GbX}$, which may be responsible for its membrane binding capacity (Blank, Wollberg, et al., 2011). The expression patterns of globin $\mathrm{X}$ in different species are distinct, as it is broadly expressed in various nonneuronal tissues in goldfish and whereas in Xenopus this protein is expressed mainly in the brain and eye (Fuchs et al., 2006; Roesner et al., 2004). However, the primary function of globin X remains elusive. 
Unlike hemoglobin or myoglobin which are penta-coordinate, GbX belongs to hexa-coordinate globins, which means that the distal histidine also coordinates to heme iron in the met and deoxy form of the protein. Previous study has shown that the distal histidine in both Ngb and Cygb is involved in regulating the affinity for gaseous ligands (Astudillo et al., 2012; Hamdane et al., 2003). Distal histidine can swing in or out of the heme binding pocket, populating either close or open conformation of the distal pocket. Such distal histidine conformational flexibility was associated with increased the heterogeneity of the distal pocket in Ngb and Cygb. Interestingly, hexa-coordinate globins shown a higher thermal stability compared to penta-coordinate globins (Hamdane et al., 2005).

Similar to both Cygb and $\mathrm{Ngb}, \mathrm{GbX}$ is also reported to be able to form disulfide bridge between two of its cytidine residues (65 and 141)(Blank, Wollberg, et al., 2011). Study has shown the disulfide bridge modulates the affinity of $\mathrm{Ngb}$ and Cygb to oxygen as well as the carbon monoxide binding kinetics through a conformational change in the E helix where the distal histidine located (Astudillo et al., 2013; Hamdane et al., 2003). Reeder's group has shown that the intramolecular disulfide bridge in Cygb is crucial for lipid binding, and Morishima's group has shown that the internal disulfide bridge in $\mathrm{Ngb}$ is vital for protein-protein interactions between $\mathrm{Ngb}$ and Gail (Beckerson, Wilson, et al., 2015; Wakasugi et al., 2003). Since the disulfide bridge can be cleaved and reformed through cellular activities, this implies that the 
reduction/oxidation of disulfide bridge in both proteins may have significant impact on protein function.

So far, only a limited number of studies were performed on GbX and the crystal structure of $\mathrm{GbX}$ is also unknown. Burmester's group has shown that both $\mathrm{Mb}$ and $\mathrm{GbX}$ transfected cell has higher viability under hypoxia while GbX promotes cell survival under conditions of oxidative stress (Koch \& Burmester, 2016). Gladwin's group has shown that $\mathrm{GbX}$ is expressed in zebrafish red blood cells, and it has a potential role as nitrite reductase in the blood (Corti et al., 2016). Unfortunately, the molecular mechanism of how GbX can carry its potential physiological functions is not clear. Since the majority of GbX's putative functions involve interactions with small diatomic ligands, in this study, we characterize $\mathrm{CO}$ binding to $\mathrm{GbX}$ as a probe to monitor how distinct structural properties of GbX, internal disulfide bridge, and distal histidine, affect $\mathrm{GbX}$ affinity and kinetics for diatomic ligands.

\subsection{Result}

\subsubsection{Oligomerization state of purified GbX}

Purification of recombinant GbX variants was confirmed by SDS-PAGE show in

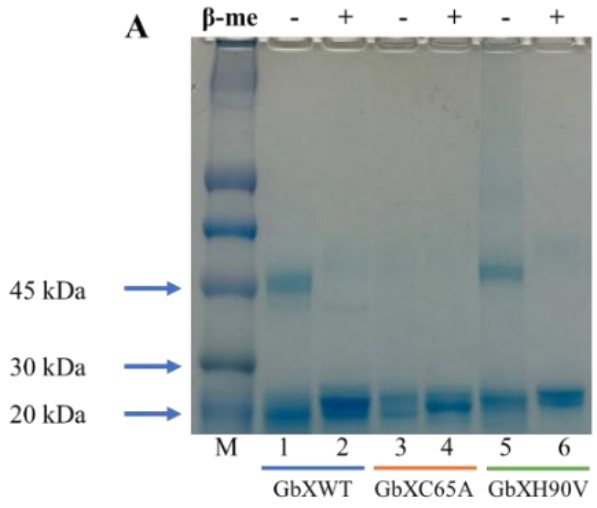




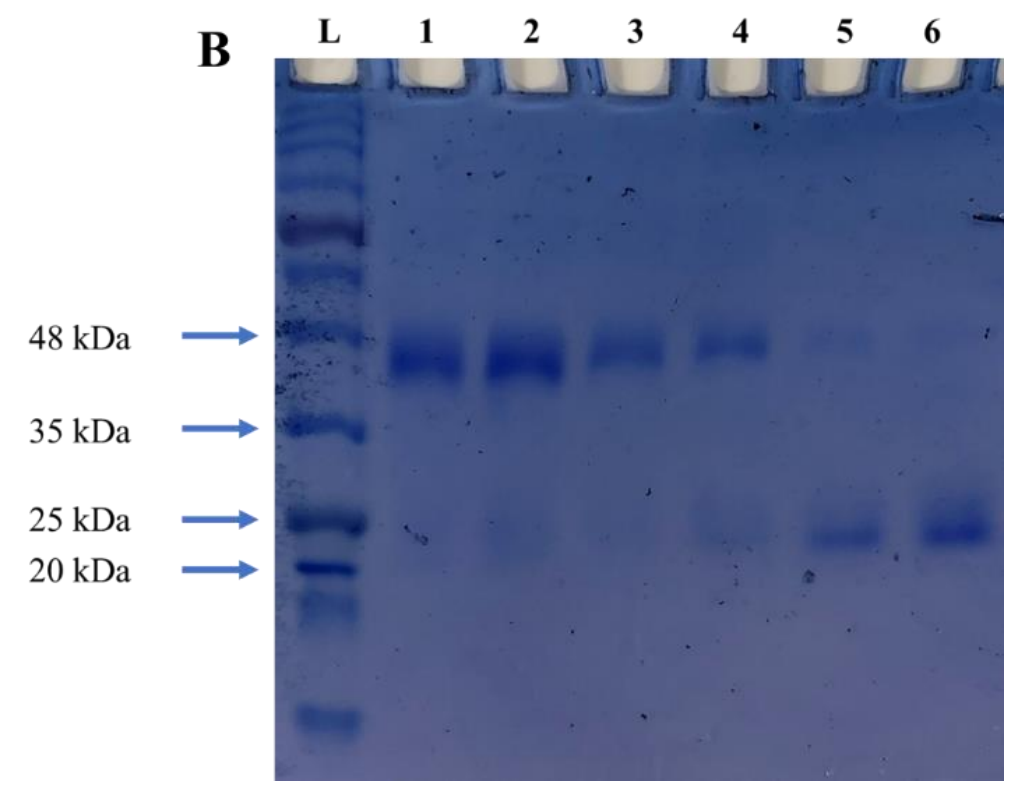

Figure 4.1. SDS-PAGE gel for purified GbX variants in the presence/absence of $\beta$-me. B, SDSPAGE gel for GbXWT fractions after separation of monomer and dimer using size exclusion chromatography.

figure 4.1 A. A band with molecular mass around $25 \mathrm{kDa}$ was observed in samples for all constructs and corresponds to the theoretical molecular mass of the GbX variants. For GbXWT and GbXH90V samples prepared in the absence of $\beta$-me, an additional band of lower intensity with a molecular mass of approximately $50 \mathrm{kDa}$ was detected in samples without $\beta$-me. The absence of $50 \mathrm{kDa}$ band in the GbXC65A mutant suggests that this band represents a covalent homodimer, with Cys65 participating in the disulfide bridge. Although the expression level of the dimer is less than the expression level of monomer, around $30 \%$, the protein samples were further purified using a size exclusion chromatography to separate oligomeric species (figure 4.1B). The results presented in this manuscript were obtained for GbX monomer, unless otherwise specified. 


\subsubsection{Steady-state UV-vis spectra and far-UV CD spectra}

The UV-vis spectra of GbX variants are similar to the spectra of other hexa-
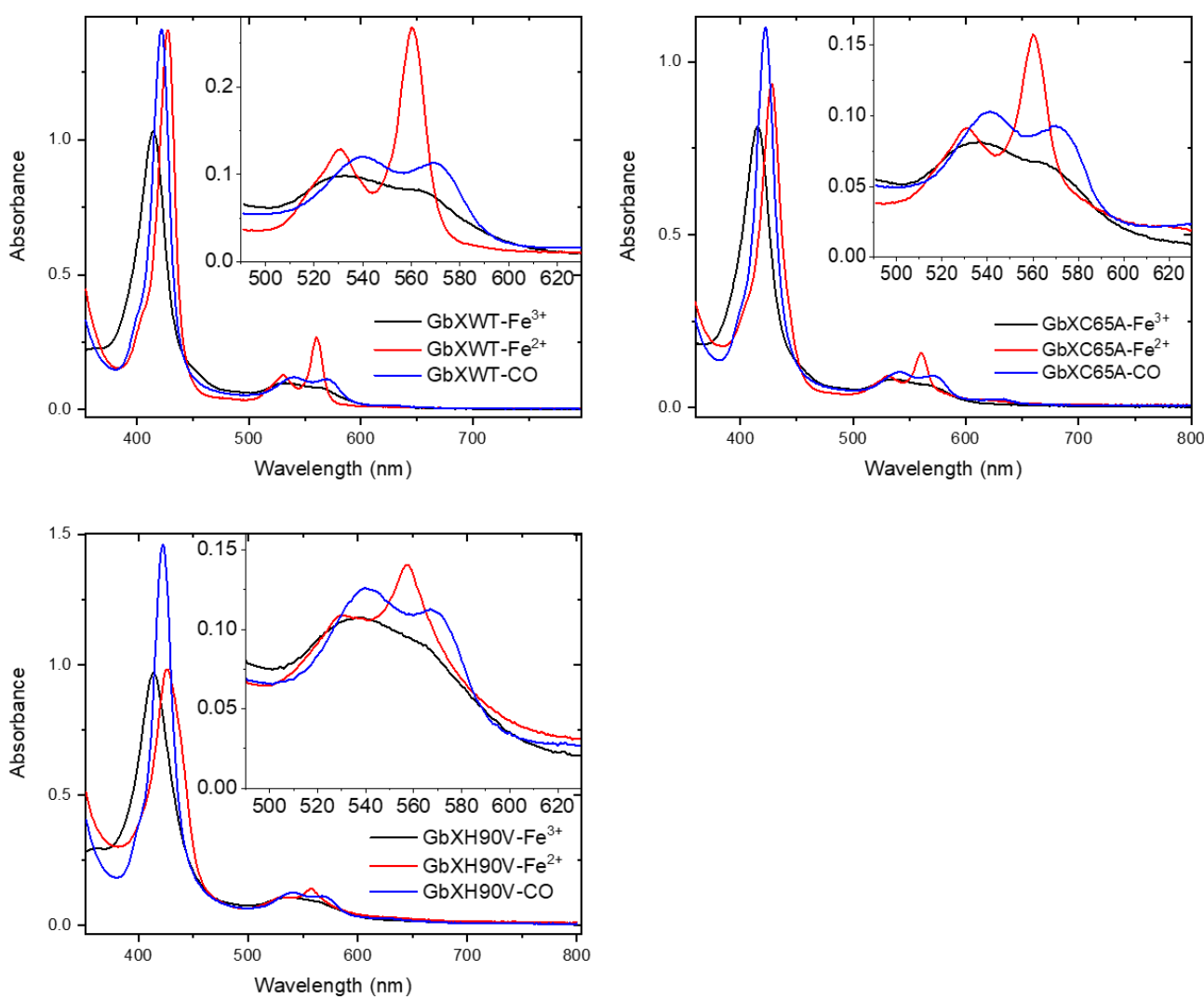

Figure 4.2. Absorbance spectra of the oxidized (met), reduced (ferrous deoxy), and CO bound forms of $\mathrm{GbX}$ variants.

coordinated globins such as Cygb and Ngb. The met-form of GbXWT shows a Soret peak at $414 \mathrm{~nm}$ and two additional Q-bands at $532 \mathrm{~nm}$ and $563 \mathrm{~nm}$ (Figure 4.2; Table 4.1). The deoxy-form of GbXWT exhibits a Soret peak at $428 \mathrm{~nm}$ and Q-bands at 531 $\mathrm{nm}$ and $560 \mathrm{~nm}$ that are characteristic for heme proteins with an intrinsic bis histidine coordination of the heme iron (Wang et al., 2003). Upon CO addition, the Soret band shifts to $422 \mathrm{~nm}$ and Q-bands appears at $539 \mathrm{~nm}$ and $569 \mathrm{~nm}$, in agreement with the formation of low-spin hexa-coordinated $\mathrm{CO}$ bound heme iron. Absorption spectrum of 
met form of GbXC65A has the Soret band at $415 \mathrm{~nm}$ but the $\beta$ band position is $4 \mathrm{~nm}$ red-shifted compared to the spectrum of met form of GbXWT, suggesting that

\begin{tabular}{|c|c|c|c|}
\hline & Soret band (nm) & $\beta$-band (nm) & a-band (nm) \\
\hline GbXWT-Fe $^{3+}$ & 414 & 532 & 563 \\
\hline GbXWT-Fe ${ }^{2+}$ & 428 & 531 & 560 \\
\hline GbXWT-CO & 422 & 539 & 569 \\
\hline GbXC65A-Fe ${ }^{3+}$ & 415 & 536 & 564 \\
\hline GbXC65A-Fe ${ }^{2+}$ & 428 & 531 & 560 \\
\hline GbXC65A-CO & 422 & 541 & 570 \\
\hline GbXH90V-Fe ${ }^{3+}$ & 414 & 536 & 565 \\
\hline GbXH90V-Fe ${ }^{2+}$ & 426 & 530 & 557 \\
\hline GbXH90V-CO & 422 & 540 & 567 \\
\hline
\end{tabular}

Table 4.1 UV-vis absorption spectra wavelength of Soret and $\alpha / \beta$ band of each GbX variants. the heme electronic structure is altered by the removal of the intra-protein disulfide bond. The absorption spectra of the deoxy- and CO bound GbXC65A constructs are nearly identical to those observed for GbXWT.

The absorption spectra measured for GbXH90A mutant do not clearly show a presence of a five-coordinate heme iron in the met- or deoxy form of the protein, although a shoulder of the Soret band and low absorbance of the $\alpha$ and $\beta$ bands observed in the deoxyGbX spectrum are consistent with a fraction of the protein being in the fivecoordinate form. A similar absorption spectrum was reported previously for NgbH64Ala mutant and attributed to the presence of a water molecule (Tejero et al., 2015). 


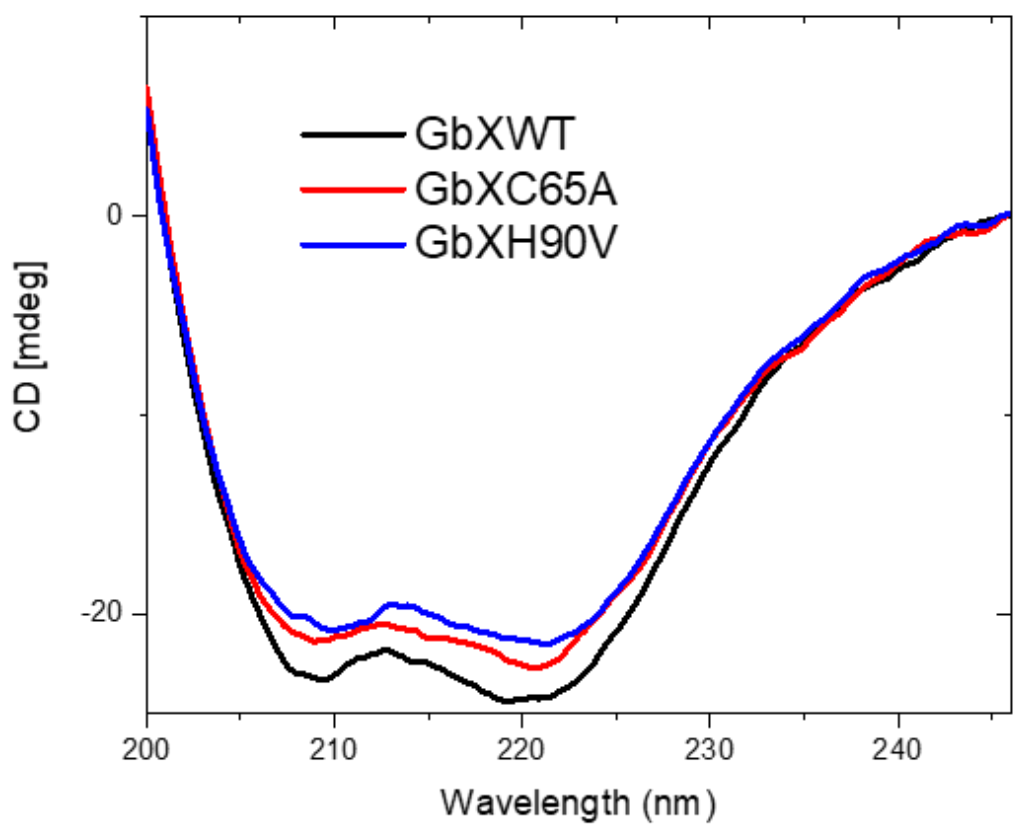

Figure 4.3. CD spectra in the far-UV region of GbX variants. All samples are aligned at $\mathrm{Abs}_{280}$ nm.

Far-UV CD spectra of all GbX variants show two prominent minima at $208 \mathrm{~nm}$ and $222 \mathrm{~nm}$ which are similar to the CD spectra recorded previously to hexa-coordiante globins and characteristic for $\alpha$-helical proteins (figure 4.3).

\subsubsection{Stability of GbX towards pH unfolding}

The stability of GbX constructs was probed by monitoring absorbance of the Soret band as a function of $\mathrm{pH}$. The overlay of the absorption spectra of met GbXWT measured in the $\mathrm{pH}$ range from 8.0 to 1.6 are shown in Figure 4.4. 


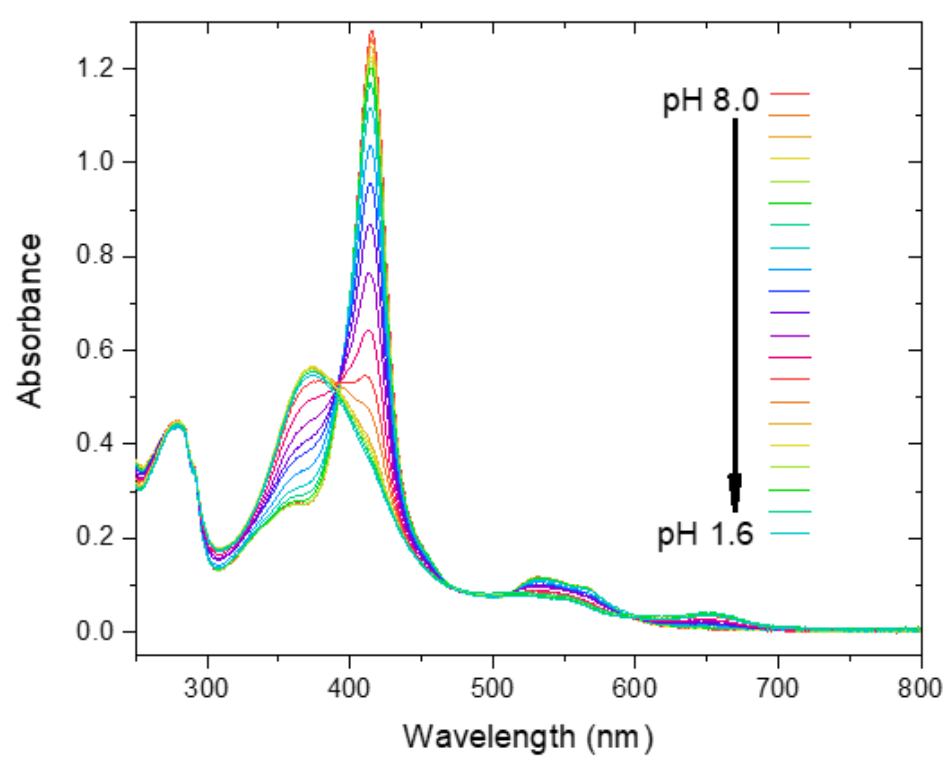

Figure 4.4. Acid-induced unfolding of GbXWT UV-vis absorption spectra. Measurements were performed in $5 \mathrm{mM}$ phosphate-citrate buffer and $100 \mathrm{mM} \mathrm{NaCl}$, under equilibrium conditions.

Figure 4.4 shows an isobestic point at $393 \mathrm{~nm}$, pointing towards a two-state release of the heme group. The absorption spectrum of GbXWT measured at $\mathrm{pH} 1.6$ shows a broad Soret band with a maximum at $372 \mathrm{~nm}$ which is consistent with a destabilize polypeptide chain and a concomitant release of the prosthetic group from the protein matrix. Analogous changes in the absorption spectra were determined for $\mathrm{pH}$ unfolding of GbXC65A and GbXH90A constructs (data not shown). The fraction of unfolded protein calculated based on the absorbance changes at $415 \mathrm{~nm}$ was plotted as a function of $\mathrm{pH}$ for all construct studied (Figure 4.5; Table 4.2) and the experimental data were analyzed using Eq.3.36. 


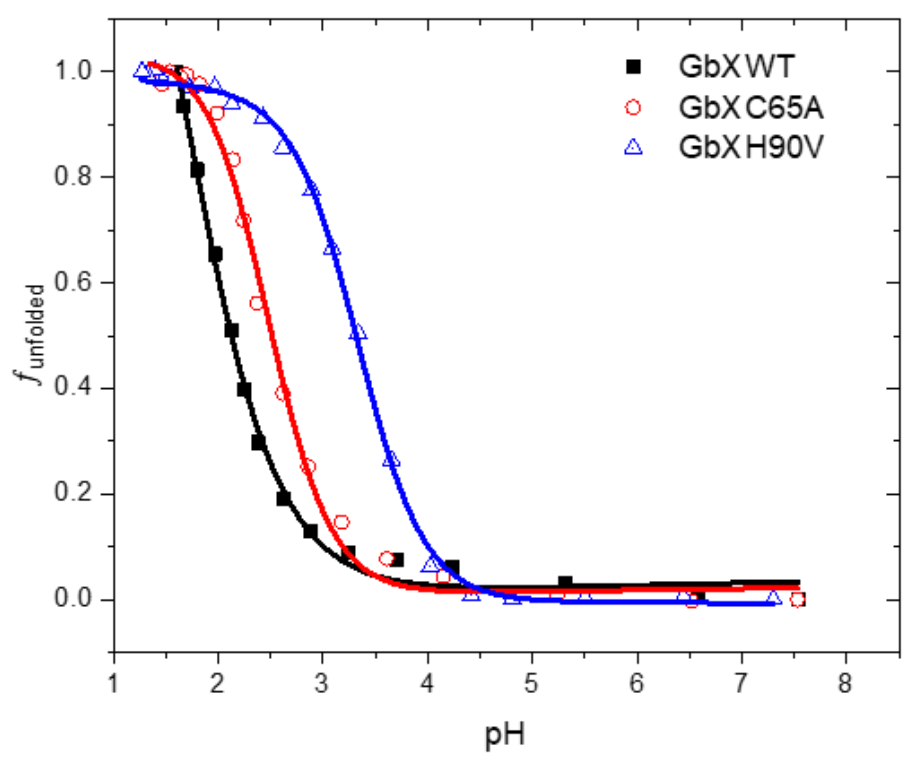

Figure 4.5. Acid-induced $\mathrm{GbX}$ variants unfolding. The solid line corresponds to the experimental data using Eq. 3.36. Measurements were performed in $5 \mathrm{mM}$ phosphate-citrate buffer and $100 \mathrm{mM} \mathrm{NaCl}$, under equilibrium conditions.

The $\mathrm{pH}$ induced unfolding of GbXWT is characterized by a $\mathrm{pH}$ at half-transition $\left(\mathrm{pH}_{\text {mid }}\right)$ of 1.9 , which is about 2.7 units lower than that previously reported for horse heart $\mathrm{Mb}\left(\mathrm{pH}_{\text {mid }}\right.$ 4.6) and 1.3 units lower than that determined for $\mathrm{Ngb}\left(\mathrm{pH}_{\text {mid }}\right.$ 3.2), indicating that the unfolding of the protein structure and release of heme in GbX occurs at more acidic $\mathrm{pH}$ range. The absence of the internal disulfide bond or the coordination bond between the distal histidine and heme iron decreases the protein stability, as the

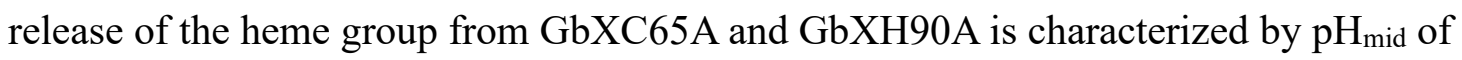
$2.48 \pm 0.01$ and $3.4 \pm 0.1$, respectively. The $\mathrm{n}$ values were found to be close to unity suggesting that the destabilization of the protein is associated with a protonation of a single amino acid residue. 


\begin{tabular}{|c|c|c|}
\hline & $\mathbf{p H}_{\text {mid }}$ & $\Delta \mathbf{V}_{\mathbf{H}^{+}}$ \\
\hline GbXWT & $1.9 \pm 0.1$ & $-1.3 \pm 0.1$ \\
\hline GbXC65A & $2.48 \pm 0.01$ & $-1.4 \pm 0.1$ \\
\hline GbXH90V & $3.4 \pm 0.1$ & $-1.2 \pm 0.2$ \\
\hline${ }^{*}$ hCygb & 3.3 & $\mathrm{~N} / \mathrm{A}$ \\
\hline${ }^{*}$ hNgb & 3.3 & $\mathrm{~N} / \mathrm{A}$ \\
\hline${ }^{*}$ hhMb & 4.6 & $\mathrm{~N} / \mathrm{A}$ \\
\hline
\end{tabular}

Table 4.2. Parameters of the $\mathrm{pH}$-induced GbX variants unfolding (* (Picotti et al., 2009)).

\subsection{4 $\mathrm{CN}^{-}$binding to $\mathrm{GbX}$}

Cyanide is frequently used as a probe to characterize the distal pocket in met form of heme proteins as it binds to the heme axial site in the met form of the heme proteins. Cyanide binding to ferric GbX leads to a red shift in the Soret band to 419 in case of GbXWT, and to $420 \mathrm{~nm}$ for the GbXC65A mutatnt as shown in Fig.6A. The equilibrium dissociation constants for $\mathrm{CN}$ - binding to met form of $\mathrm{GbX}$ constructs are determined by monitoring the changes in the absorption spectra upon addition of small aliquots of $\mathrm{CN}$ - stock solution.

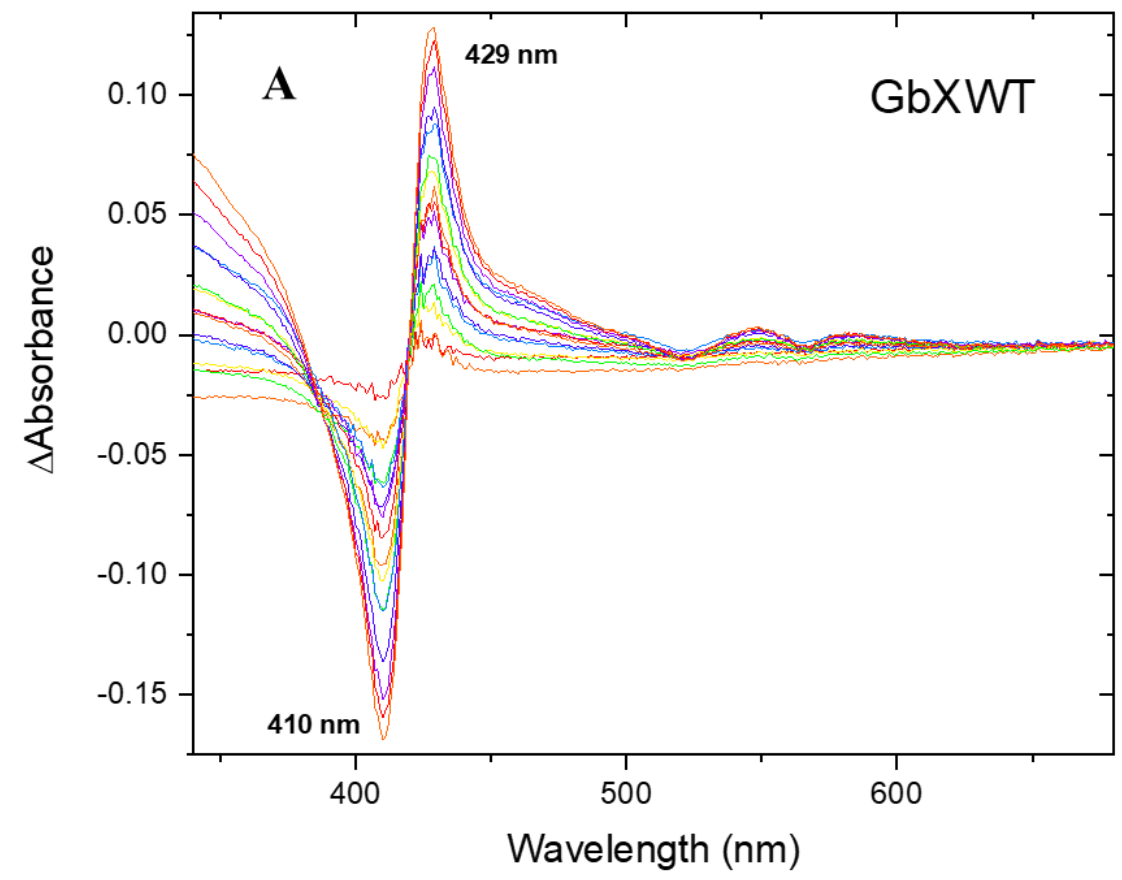




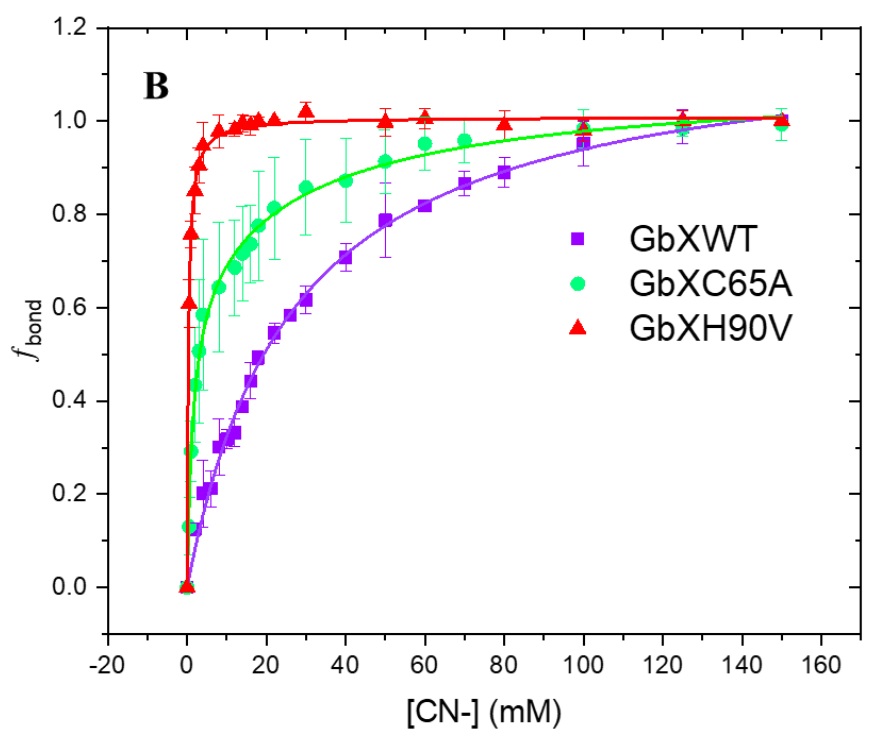

Figure 4.6. A, absorption difference spectra at various cyanide concentrations, relative to the ferric $\mathrm{GbX}$ form (without cyanide), the spectra were measured at room temperature in $50 \mathrm{mM}$ TrisHCl at $\mathrm{pH}$ 7. B, cyanide binding to $\mathrm{GbX}$ variants, fraction bond was calculated by absorption at $410 \mathrm{~nm}$ as a function of cyanide concentration, only cysteine mutant shows possible two binding sites.

The fraction of protein in the $\mathrm{CN}^{-}$bound form was calculated based on the absorbance at $410 \mathrm{~nm}$ at various $\mathrm{CN}^{-}$concentrations (Figure $4.6 \mathrm{~A}$ ) and the plots of the fraction of proteins in the $\mathrm{CN}^{-}$bound form as a function of cyanide concentrations are shown in figure 4.6 B. The titration curves for GbXWT and GbXH90V were analyzed using a single binding model whereas of the titration curve for GbXC65A construct was fitted using a two independent binding site model, as the single binding site model have not provided a satisfactory fit. The $\mathrm{CN}^{-}$binds to GbXWT and GbXH90V mutant with an equilibrium dissociation constant, $\mathrm{K}_{\mathrm{d}}=27 \pm 1 \mathrm{mM}$ and $0.3 \pm 0.1 \mathrm{mM}$ respectively. Interestingly, GbXWT has10,000-fold lower affinity for CN- than previously reported affinity of $\mathrm{CN}^{-}$association to Cygb. Unlike GbXWT and GbxH90V mutant, titration of GbXC65A mutant shown two cyanide binding sites, a high affinity site $\left(\mathrm{K}_{\mathrm{d}}=1.5 \pm 0.4\right.$ $\mathrm{mM})$ and a low affinity site $\left(\mathrm{K}_{\mathrm{d}}=38 \pm 4 \mathrm{mM}\right)$. 


\begin{tabular}{|c|c|c|}
\hline & $\mathbf{K}_{\mathrm{d}} \mathbf{1}(\mathbf{m M})$ & $\mathbf{K}_{\mathrm{d}} \mathbf{2}(\mathbf{m M})$ \\
\hline GbXWT & $27 \pm 1$ & $\mathrm{~N} / \mathrm{A}$ \\
\hline GbXC65A & $1.5 \pm 0.4$ & $38 \pm 4$ \\
\hline GbXH90V & $0.3 \pm 0.1$ & $\mathrm{~N} / \mathrm{A}$ \\
\hline thCygbWT & 0.0028 & 0.32 \\
\hline †hMbWT & 1.7 & N/A \\
\hline
\end{tabular}

Table 4.3. Parameters of the $\mathrm{CN}^{-}$binding to $\mathrm{GbX}$ variants (†(Tsujino et al., 2014), $\neq($ Dou et al., 1996)).

\subsubsection{GbX CO binding kinetic}

To further analyze interactions of $\mathrm{GbX}$ variants with diatomic ligand, $\mathrm{CO}$ binding kinetics were determine using transient absorption spectroscopy. The transient absorption traces for CO binding to GbX constructs studied are shown in Figure 4.7 A. The traces were analyzed using both multiple-exponential decay model and MEM (maximum entropy method) and the kinetic parameters are summarized in Table 4.4. Four distinct kinetics were resolved for $\mathrm{CO}$ association to GbXWT: $\mathrm{k}_{1}=4 \pm 0.3 \mu \mathrm{M}^{-1} \mathrm{~s}^{-}$ ${ }^{1} \mathrm{k}_{2}=0.87 \pm 0.09 \mu \mathrm{M}^{-1} \mathrm{~s}^{-1}, \mathrm{k}_{3}=0.49 \pm 0.06 \mu \mathrm{M}^{-1} \mathrm{~s}^{-1}$ and $\mathrm{k}_{4}=0.08 \pm 0.02 \mu \mathrm{M}^{-1} \mathrm{~s}^{-1}$ although the amplitude associate with the slowest rate constant is small, $\sim 5 \%$. Similar rate constants were obtained using MEM analysis. The analysis of the CO rebinding kinetics to GbXC65A mutant reveals three kinetics with the rate constants similar to $\mathrm{k}_{2}$, $\mathrm{k}_{3}$ and $\mathrm{k}_{4}$ found for $\mathrm{CO}$ rebinding to GbXWT. The slowest process characterized by $\mathrm{k}_{3}$ has a small amplitude, $\sim 4 \%$. The substitution of distal His 90 by Ala results in significantly faster CO rebinding with the first two rate constants being faster than those 

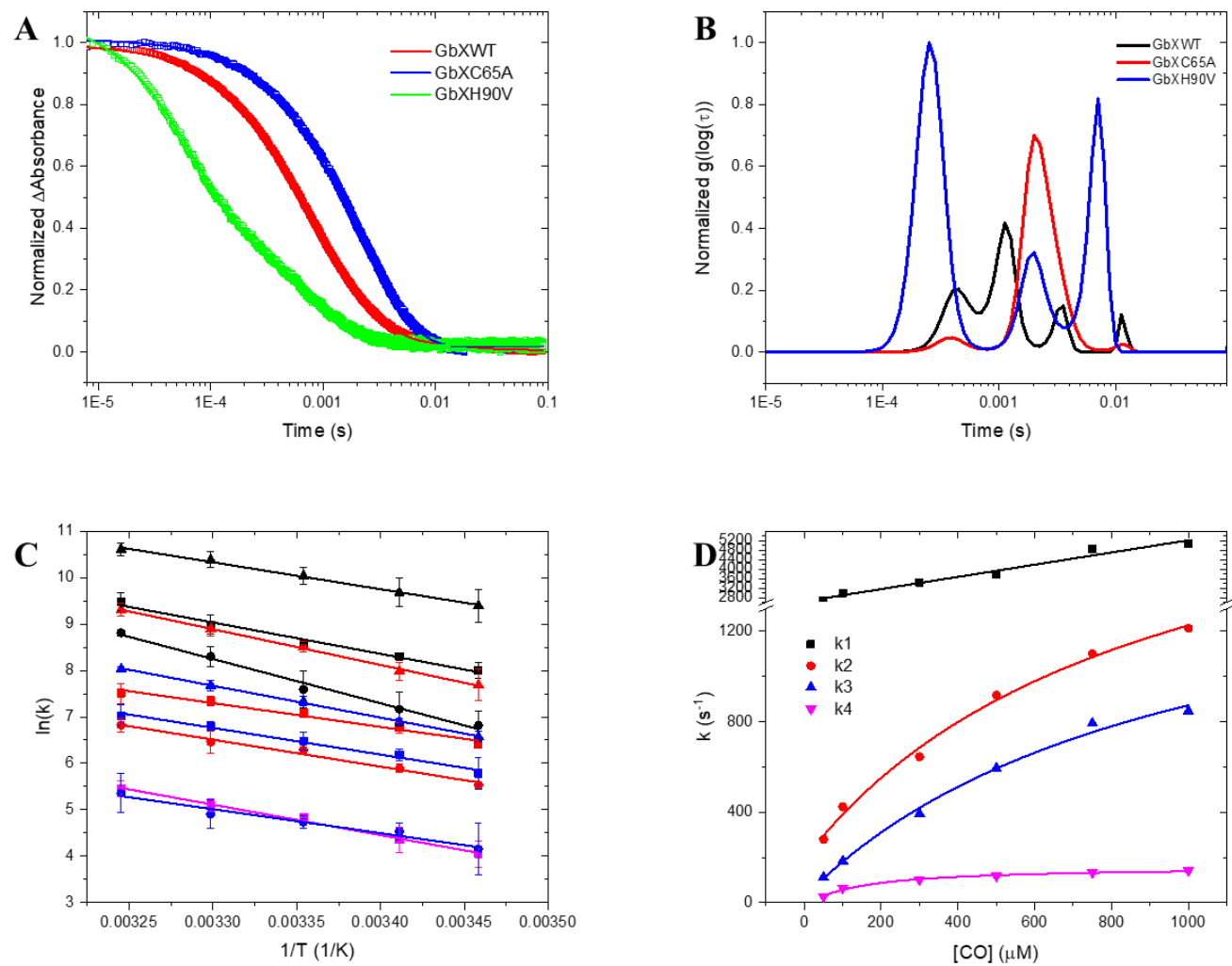

Figure 4.7. A, transient absorption traces for $\mathrm{CO}$ rebinding to $\mathrm{GbX}$ variants. $\mathrm{B}$, Lifetime distribution associated with the $\mathrm{CO}$ rebinding to $\mathrm{GbX}$ variant determined by MEM approach. $\mathrm{C}$, Arrhenius plot of temperature dependent $\mathrm{CO}$ rebinding to $\mathrm{GbX}$ variants, color represent different rate constant (black k1, red k2, blue k3, and purple k4) and symbol represent different $\mathrm{GbX}$ variants (square WT, round C65A, and triangle H90V). D, Eyring plot of temperature dependent $\mathrm{CO}$ rebinding to $\mathrm{GbX}$ variants.

observed in the GbXWT and GbXC65A mutant whereas the third rate constant, $\mathrm{k}_{3}=1$ $\pm 0.06 \mu \mathrm{M}^{-1} \mathrm{~s}^{-1}$ is very similar to the value of $\mathrm{k}_{2}$ for ligand binding to GbXWT. Comparing the averaged rate constant $\left(\mathrm{k}_{\mathrm{ave}}\right)$ of $\mathrm{GbX}$ variants, the average rate constant for $\mathrm{CO}$ rebinding to $\mathrm{GbXWT}$ is $1.4 \mu \mathrm{M}^{-1} \mathrm{~s}^{-1}$ which is around three fold larger than the

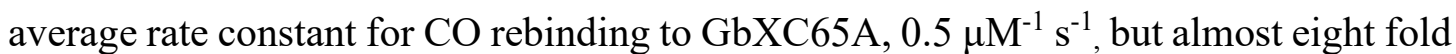
smaller than the average rate constant for $\mathrm{CO}$ binding to GbXH90V, $10.4 \mu \mathrm{M}^{-1} \mathrm{~s}^{-1}$. These results indicate the internal disulfide bridge and distal histidine play a role in modulating the ligand binding to GbX. Interestingly, the $\mathrm{k}_{\mathrm{ave}}$ of GbXWT is close to the 
$\mathrm{k}_{\mathrm{ave}}$ of $\mathrm{hhMb}$ but significantly smaller than the rate constant for $\mathrm{CO}$ binding to rice $\mathrm{Hb}$ as well as hCygb. From the temperature dependence of the individual rate constants,

\begin{tabular}{|c|c|c|c|c|c|c|c|c|c|}
\hline & $\begin{array}{c}\mathrm{A}_{1} \\
(\%)\end{array}$ & $\begin{array}{c}\mathrm{k}_{1} \\
\left(\mu \mathrm{M}^{-1} \mathrm{~s}^{-1}\right)\end{array}$ & $\begin{array}{c}\mathrm{A}_{2} \\
(\%)\end{array}$ & $\begin{array}{c}\mathrm{k}_{2} \\
\left(\mu \mathrm{M}^{-1} \mathrm{~s}^{-1}\right)\end{array}$ & $\begin{array}{c}\mathrm{A}_{3} \\
(\%)\end{array}$ & $\begin{array}{c}\mathrm{k}_{3} \\
\left(\mu \mathrm{M}^{-1} \mathrm{~s}^{-1}\right)\end{array}$ & $\begin{array}{c}\mathrm{A}_{4} \\
(\%)\end{array}$ & $\begin{array}{c}\mathrm{k}_{4} \\
\left(\mu \mathrm{M}^{-1} \mathrm{~s}^{-1}\right)\end{array}$ & $\begin{array}{c}\mathrm{k}_{\text {ave }} \\
\left(\mu \mathrm{M}^{-1} \mathrm{~S}^{-1}\right)\end{array}$ \\
\hline $\begin{array}{c}\text { GbXWT } \\
(\text { decy })\end{array}$ & $21 \pm 2$ & $4.0 \pm 0.3$ & $57 \pm 3$ & $0.87 \pm 0.09$ & $17 \pm 1$ & $0.49 \pm 0.06$ & $5 \pm 2$ & $0.08 \pm 0.02$ & 1.4 \\
\hline $\begin{array}{c}\text { GbXWT } \\
(\mathrm{MEM})\end{array}$ & $35 \pm 2$ & $2.3 \pm 0.07$ & $47 \pm 2$ & $0.83 \pm 0.05$ & $12 \pm 0.3$ & $0.24 \pm 0.06$ & $6 \pm 0.5$ & $0.06 \pm 0.03$ & 1.2 \\
\hline $\begin{array}{c}\text { GbXC65A } \\
(\text { decy })\end{array}$ & $15 \pm 4$ & $1.4 \pm 0.5$ & $81 \pm 3$ & $0.36 \pm 0.03$ & $4 \pm 3$ & $0.09 \pm 0.02$ & N/A & N/A & 0.5 \\
\hline $\begin{array}{c}\text { GbXC65A } \\
(\mathrm{MEM})\end{array}$ & $5 \pm 2$ & $2.9 \pm 0.4$ & $94 \pm 2$ & $0.39 \pm 0.07$ & $2 \pm 0.5$ & $0.06 \pm 0.03$ & N/A & N/A & 0.5 \\
\hline $\begin{array}{c}\text { GbXH90V } \\
(\text { decy })\end{array}$ & $56 \pm 1$ & $16.9 \pm 5.0$ & $22 \pm 4$ & $3.0 \pm 0.6$ & $22 \pm 4$ & $1 \pm 0.06$ & N/A & N/A & 10.4 \\
\hline $\begin{array}{c}\text { GbXH90V } \\
(\mathrm{MEM})\end{array}$ & $46 \pm 18$ & $29.1 \pm 10.5$ & $30 \pm 13$ & $6.0 \pm 5.5$ & $24 \pm 9$ & $1.1 \pm 0.2$ & N/A & N/A & 15.4 \\
\hline$*$ hhMb & N/A & N/A & N/A & N/A & N/A & N/A & N/A & N/A & 0.5 \\
\hline trHb & N/A & N/A & N/A & N/A & N/A & N/A & N/A & N/A & 5.0 \\
\hline fhCygb & 1.4 & 325 & 98.6 & 7.5 & N/A & N/A & N/A & N/A & 11.9 \\
\hline
\end{tabular}

Table 4.4. Rate constants for $\mathrm{CO}$ binding to GbX variants obtained by fitting the experimental data by exponential decay model and MEM analysis at $20^{\circ} \mathrm{C}$. (*(Belogortseva et al., 2007), $\uparrow$ (Butcher et al., 2017), $\ddagger$ (Astudillo et al., 2013) )

the activation energy, log pre-exponential factor, reaction enthalpy, and reaction entropy was extrapolated (figure $4.7 \mathrm{C}$ and table 4.5). The activation energy for $\mathrm{CO}$ binding to $\mathrm{GbX}$ variants is similar within the experimental error for all variants studied suggesting the similar activation barrier for CO binding to heme iron, $\sim 10 \mathrm{kcal} \mathrm{mol}^{-1}$. This activation barrier is higher than the barrier for $\mathrm{CO}$ binding to horse heart $\mathrm{Mb}(8.5 \mathrm{kcal}$ $\left.\mathrm{mol}^{-1}\right)$ and human $\mathrm{Ngb}\left(4.5 \mathrm{kcalmol}^{-1}\right)$. Analogously, activation enthalpy for CO binding to $\mathrm{GbX}, \Delta \mathrm{H}^{\#}=10 \mathrm{kcal} \mathrm{mol}^{-1}$ is slightly increased compare to that observed for $\mathrm{CO}$ binding to horse heart $\mathrm{Mb}\left(\sim 7.1 \mathrm{kcal} \mathrm{mol}^{-1}\right)$ and rice $\mathrm{Hb}\left(\sim 8 \mathrm{kcal} \mathrm{mol}^{-1}\right)$. Notably, the overall activation entropy for $\mathrm{CO}$ binding to GbXWT is higher than that determined for 
$\mathrm{hhMb}$ and $\mathrm{rHb}$, suggesting distinct structural reorganization upon $\mathrm{CO}$ binding to $\mathrm{GbX}$ compare to other globins.

\begin{tabular}{|c|c|c|c|c|c|}
\hline & & $\begin{array}{c}\mathrm{E}_{\mathrm{a}} \\
\left(\mathrm{kcal} \mathrm{mol}^{-1}\right)\end{array}$ & $\log (A)$ & $\begin{array}{c}\Delta \mathrm{H}^{\ddagger} \\
\left(\mathrm{kcal} \mathrm{mol}^{-1}\right)\end{array}$ & $\begin{array}{c}\Delta \mathrm{S}^{\ddagger} \\
\left(\mathrm{cal} \mathrm{mol} \mathrm{l}^{-1} \mathrm{~K}^{-1}\right)\end{array}$ \\
\hline \multirow{4}{*}{ GbXWT } & $\mathrm{k} 1$ & $13.5 \pm 3.3$ & $13.7 \pm 2.5$ & $11.0 \pm 1.9$ & $-4.3 \pm 6$ \\
\hline & $\mathrm{k} 2$ & $10.3 \pm 2.4$ & $10.6 \pm 1.8$ & $12.2 \pm 5.4$ & $-3.4 \pm 18$ \\
\hline & $\mathrm{k} 3$ & $11.2 \pm 4.4$ & $11.1 \pm 3.2$ & $12.8 \pm 4.3$ & $-2.5 \pm 14$ \\
\hline & $\mathrm{k} 4$ & $12.8 \pm 4.6$ & $11.5 \pm 3.3$ & $14.2 \pm 4.6$ & $-1.2 \pm 15$ \\
\hline \multirow{3}{*}{$\mathrm{GbXC65A}$} & $\mathrm{k} 1$ & $18.6 \pm 3.3$ & $17.0 \pm 2.3$ & $18.0 \pm 3.3$ & $16.8 \pm 10$ \\
\hline & $\mathrm{k} 2$ & $11.7 \pm 1.9$ & $11.4 \pm 1.6$ & $11.1 \pm 1.9$ & $-8.9 \pm 6$ \\
\hline & $\mathrm{k} 3$ & $10.0 \pm 6.4$ & $9.4 \pm 4.7$ & $10.3 \pm 6.4$ & $-14.2 \pm 21$ \\
\hline \multirow{3}{*}{$\mathrm{GbXH} 90 \mathrm{~V}$} & $\mathrm{k} 1$ & $11.2 \pm 2.1$ & $12.6 \pm 1.4$ & $10.6 \pm 2.1$ & $-2.9 \pm 6.4$ \\
\hline & $\mathrm{k} 2$ & $15.0 \pm 1.9$ & $14.6 \pm 1.4$ & $14.4 \pm 1.9$ & $6.7 \pm 5.8$ \\
\hline & $\mathrm{k} 3$ & $13.6 \pm 1.5$ & $13.1 \pm 1.1$ & $14.3 \pm 0.9$ & $2.6 \pm 4.7$ \\
\hline$*_{\mathrm{rHb}} \mathrm{WT}$ & $\mathrm{k}$ & $8.5 \pm 0.4$ & N/A & $8.0 \pm 0.4$ & $-28 \pm 1.3$ \\
\hline thhMb & $\mathrm{k}$ & N/A & N/A & $7.1 \pm 0.8$ & $-22.4 \pm 2.8$ \\
\hline
\end{tabular}

Table 4.5. Activation energy, log pre-exponential factor, activation enthalpy, and activation entropy of temperature dependent CO rebinding to GbX variants. (*(Butcher et al., 2017), †(Mikšovská et al., 2003))

The rates for $\mathrm{CO}$ association to $\mathrm{GbX}$ were also monitored as a function of $\mathrm{CO}$ concentration and the plots of the apparent rate constants as a function of $\mathrm{CO}$ concentration are shown in Figure 4.8. For CO binding to GbXH90V mutant, all three rate constants exhibit a linear dependence on the ligand concentration in agreement with one step CO binding. The experimental data were analyzed using equation 3.45 


$$
k_{a p p}=k_{o n}[C O]+k_{o f f}
$$

where $\mathrm{k}_{\mathrm{app}}$ is the observed rate constant for the formation of the CO-GbX complex, $\mathrm{k}_{\mathrm{on}}$ and $\mathrm{k}_{\text {off }}$ are the elementary rate constants. The individual rate constants are summarized in Table 4.6 and were used calculate the equilibrium dissociation constants, $\mathrm{K}_{\mathrm{D}}$, for each individual step.
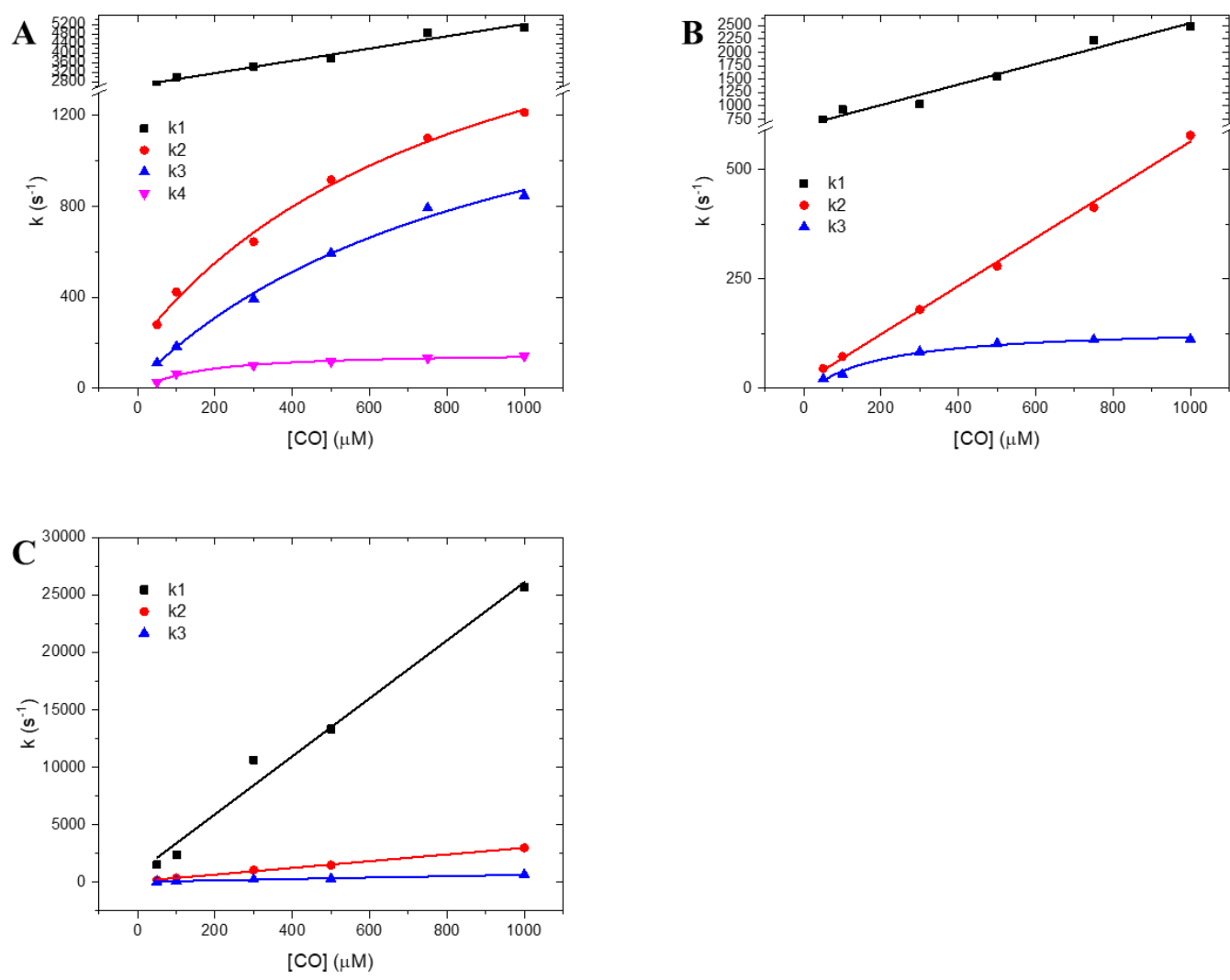

Figure 4.8. Logarithmic plots of the rate constants for CO rebinding to GbXWT (A), GbXC65A (B), and $\mathrm{GbXH} 90 \mathrm{~V}(\mathrm{C})$ as a function of $\mathrm{CO}$ concentration, experiment was performed by 20 $\mu \mathrm{M}$ protein in $50 \mathrm{mM}$ TrisHCl, $\mathrm{pH} 7$, at $25^{\circ} \mathrm{C}$.

The plot of the apparent rate constants for the ligand binding to GbXWT and GbXC65A mutant are more complex than for GbXH90V mutant. In case of $\mathrm{CO}$ binding

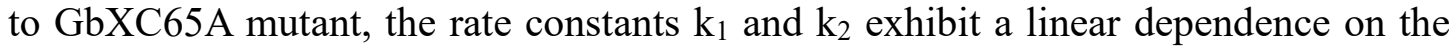


$\mathrm{CO}$ concentration, in agreement with the $\mathrm{CO}$ directly binding to a five-coordinate heme iron in a single step reaction, in a similar way as observed for GbXH90V mutant.

\begin{tabular}{|c|c|c|c|c|}
\hline & $k_{o n}^{C O}\left(\mu \mathrm{M}^{-1} \mathrm{~s}^{-1}\right)$ & $k_{o f f}^{C O}\left(\mathrm{~s}^{-1}\right)$ & $\mathrm{K}_{\mathrm{dCO}}(\mu \mathrm{M})$ & \\
\hline GbXWT k1 & $2.6 \pm 0.2$ & $2639 \pm 128$ & $1024.8 \pm 128$ & \\
\hline GbXC65A k1 & $1.9 \pm 0.2$ & $636 \pm 91$ & $344 \pm 84$ & \\
\hline GbXC65A k2 & $0.55 \pm 0.01$ & $14 \pm 8$ & $26 \pm 15$ & \\
\hline GbXH90V k1 & $25 \pm 2$ & $859 \pm 992$ & $38 \pm 43$ & \\
\hline GbXH90V k2 & $2.9 \pm 0.1$ & $80 \pm 51$ & $29 \pm 19$ & \\
\hline GbXH90V k3 & $0.62 \pm 0.03$ & $27 \pm 17$ & $45 \pm 30$ & \\
\hline \multirow[t]{2}{*}{$\# \mathrm{Mb}$} & 0.53 & 0.019 & 0.03 & \\
\hline & $k_{o n}^{C O}\left(\mu \mathrm{M}^{-1} \mathrm{~s}^{-1}\right)$ & $k_{o n}^{H i s}\left(\mathrm{~s}^{-1}\right)$ & $k_{o f f}^{H i s}\left(\mathrm{~s}^{-1}\right)$ & $\mathrm{K}_{\mathrm{aHis}}$ \\
\hline GbXWT k2 & 1122 & 382989 & 1585 & 241 \\
\hline GbXWT k3 & 79 & 63033 & 1577 & 79 \\
\hline GbXWT k4 & 105 & 20799 & 168 & 104 \\
\hline GbXC65A k3 & 21 & 5630 & 151 & 37 \\
\hline$*_{\mathrm{rHb}}$ & 6.8 & 75 & 40 & 1.9 \\
\hline *Syn $\mathrm{Hb}$ & 90 & 4200 & 14 & 300 \\
\hline${ }^{*} \mathrm{hNgb}$ & 40 & $>2000$ & 2.3 & $\sim 1000$ \\
\hline *hCygb & 5.6 & 430 & 0.5 & 860 \\
\hline
\end{tabular}

Table 4.6. Rate and equilibrium constants for CO binding to GbX variants. (\#(Rohlfs et al., 1990), *(Smagghe et al., 2006))

From the linear fit of the experimental data, the values of $k_{\text {on }}$ and $k_{\text {off }}$ for each individual binding step were extrapolated (Table 4.6). Interestingly, the equilibrium dissociation constant for $\mathrm{CO}$ association to GbXC65A mutant, that was determined based on the $k_{\text {on }}$ and $k_{\text {off }}$ values for the second kinetic step, $K_{D}=26 \pm 15 \mu \mathrm{M}$, is comparable to that observed for $\mathrm{CO}$ binding to distal histidine mutant. On the other 
hand, the equilibrium dissociation constant determined from the individual rate constants for the first step is about ten times larger than that determined from the individual rate constants for the second step or the equilibrium dissociation constant for CO binding to GbXC65A mutant. In case of WT, the plot of the $\mathrm{k}_{\mathrm{obs}}$ as a function of the $\mathrm{CO}$ concentration is linear only for $\mathrm{k}_{1}$. As described previously, the $\mathrm{k}_{\mathrm{on}}$ and $\mathrm{k}_{\text {off }}$ values were extrapolated from the linear fit of the experimental data and used to determine the equilibrium dissociation constant, $\mathrm{K}_{\mathrm{D}}=1024 \pm 128 \mu \mathrm{M}$. The saturation plots of $\mathrm{k}_{\text {obs }}$ as a function of $\mathrm{CO}$ concentration observed for GbXWT and GbXC65A mutant indicate, that the $\mathrm{CO}$ binding occurs as a two step process and can be described according to Scheme 1:

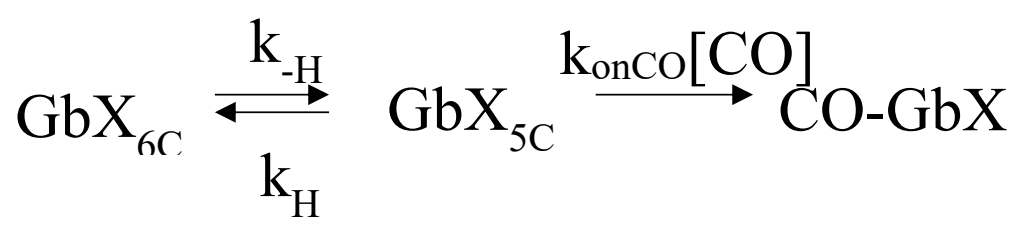

$\mathrm{k}_{-\mathrm{H}}$ and $\mathrm{k}_{\mathrm{H}}$ are the individual rate constant for the distal histidine dissociation and binding, respectively, and $\mathrm{k}_{\mathrm{onCO}}$ is the rate for $\mathrm{CO}$ binding. Using steady-state approximation approach, the observed rate constant depends on the ligand concentration according to equation 3.46:

$$
k_{o b s, C O}=\frac{k_{-H} k_{o n}^{C O}[\mathrm{CO}]}{k_{H}+k_{-H}+k_{o n}^{C O}[\mathrm{CO}]}
$$

The results of the analysis of the rate constant using Eq 3.46 are provided in Table 4.6. The affinity constant of distal histidine $\left(\mathrm{K}_{\mathrm{aHis}}\right)$ to $\mathrm{GbXWT}$ is around 140 which is almost four-fold higher than the affinity constant for the distal histidine in GbXC65A, 
suggesting that the disruption of the internal disulfide bridge further decreases the affinity of distal histidine to heme iron. Surprisingly, the $k_{o n}^{C O}$ and $\mathrm{K}_{\mathrm{aHis}}$ determined for GbXWT are very close to these values measured for pant hexa-coordinate globin, SynHb. These data suggest that GbX interacts with $\mathrm{CO}$ in a similar way as observed previously in some plants hemoglobins.

\subsubsection{CO dissociation from $\mathrm{GbX}$}

The quantum yield for bimolecular $\mathrm{CO}$ dissociation from $\mathrm{CO}-\mathrm{GbX}$ was determined as described in material and methods. The observed quantum yields are similar to quantum yields determined previously for $\mathrm{CO}$ dissociation from $\mathrm{CO}-\mathrm{Ngb}$ and CO-Cygb and for all constructs studied the bimolecular quantum yield is temperature dependent (Figure 4.9). The quantum yield of $\mathrm{CO}$ from $\mathrm{GbX}$ variants are temperature dependent and ranges from 0.55 to 0.65 between $16^{\circ} \mathrm{C}$ to $35^{\circ} \mathrm{C}$ in case of GbX WT.

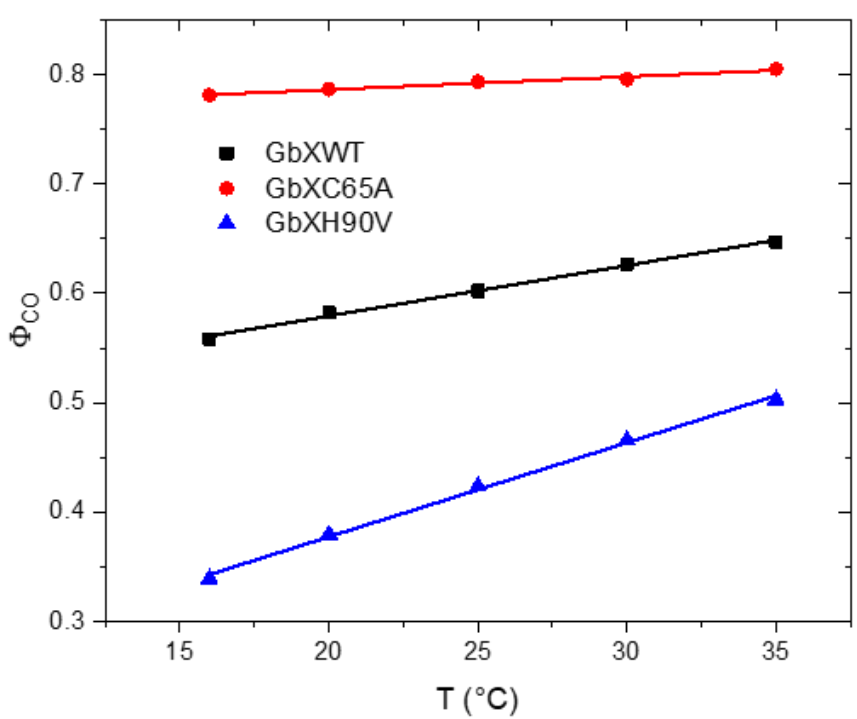

Figure 4.9. CO quantum yield for bimolecular dissociation from $\mathrm{GbX}$ variants as a function of temperature. 
In contrast, the quantum yield for bimolecular $\mathrm{CO}$ dissociation from $\mathrm{GbXC65}$ is higher and ranges from 0.78 to 0.80 . These results suggest that the presence of the internal disulfide bond decreases the internal barrier for $\mathrm{CO}$ geminate rebinding. The quantum yield for the $\mathrm{CO}$ bimolecular dissociation from $\mathrm{CO}$ GbXH90V is smaller and ranges from 0.33 to 0.50 in the temperature range studied. These results suggest that the absence of the distal histidine residue significantly facilitate the $\mathrm{CO}$ geminate rebinding.

Photo-acoustic calorimetry experiments were conducted to investigate the reaction enthalpy and volume changes upon the dissociation of $\mathrm{CO}$ from $\mathrm{GbX}$ variants. Figure 4.10 show the overlay of normalized acoustic traces of $\mathrm{CO}$ bound $\mathrm{GbX}$ variants and the reference. There is significant phase shift between the sample acoustic wave and
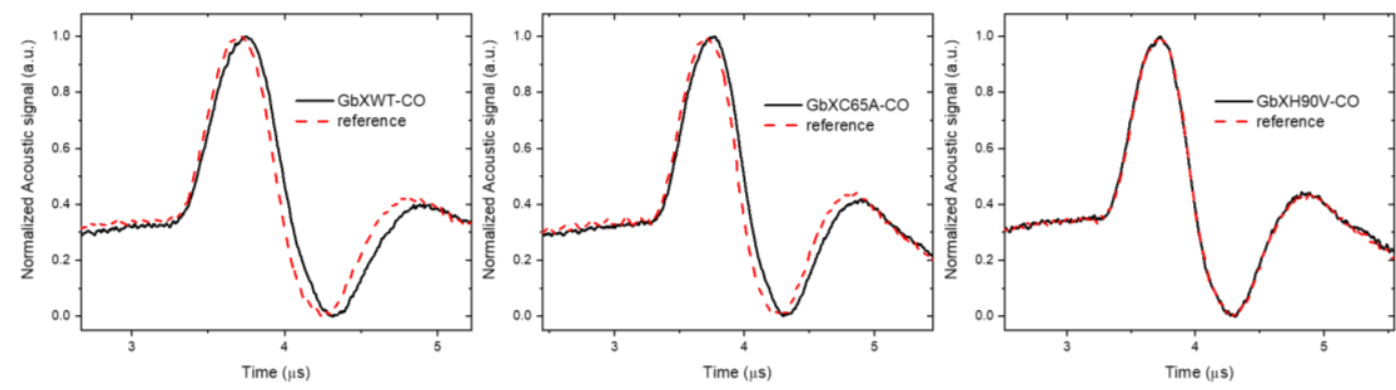

Figure 4.10. Overlay of normalized acoustic traces of $\mathrm{CO}$ bound $\mathrm{GbX}$ variants with reference. reference acoustic wave in both GbXWT and GbXC65A, suggesting that the dissociation of $\mathrm{CO}$ from the heme pocket is a multi-step process. No phase shift was observed for GbXH90V, indicating fast $\mathrm{CO}$ escape from the distal cavity ( $\tau<50 \mathrm{~ns}$ ) following the breakage of the Fe-CO bond. Deconvolution of the acoustic traces reveals two distinct phases for $\mathrm{CO}$ escape from GbXWT and GbXC65A: 1$)$ a prompt phase $\left(\tau_{1}\right.$ 
$<50 \mathrm{~ns}$ ) which represents the photo-cleavage of the CO-Fe bond and ligand relocation within the distal cavity and possibly its migration into distant hydrophobic cavities, and 2) a kinetic step with a lifetime $\tau_{2}=137 \mathrm{~ns}$ at $20^{\circ} \mathrm{C}$ that can be associate with $\mathrm{CO}$ escape into the surrounding solvent. The activation thermodynamic parameter $\left(\Delta \mathrm{H}^{\#}\right.$ and $\left.\Delta \mathrm{V}^{\#}\right)$ for $\mathrm{CO}$ release from $\mathrm{GbX}$ variants were obtained using Eyring plot (Figure 4.11) for $\tau_{2}$ parameters. Table 4.7 summarizes the observed values for the reaction and activation parameters associated with the photo-dissociation of Fe-CO bond and subsequent ligand escape from the protein matrix in $\mathrm{GbX}$ variants as well as previously reported data for Cygb and $\mathrm{Mb}$. The activation enthalpy for $\mathrm{CO}$ escape from the protein matrix in GbXWT and Cys65 mutant are very similar to Mb and CygbWT pointing towards a similar activation barrier for $\mathrm{CO}$ escape from globins. However, the activation entropy of $\mathrm{CO}$ escape from GbXWT and Cys65 mutant is higher than Mb and CygbWT imply
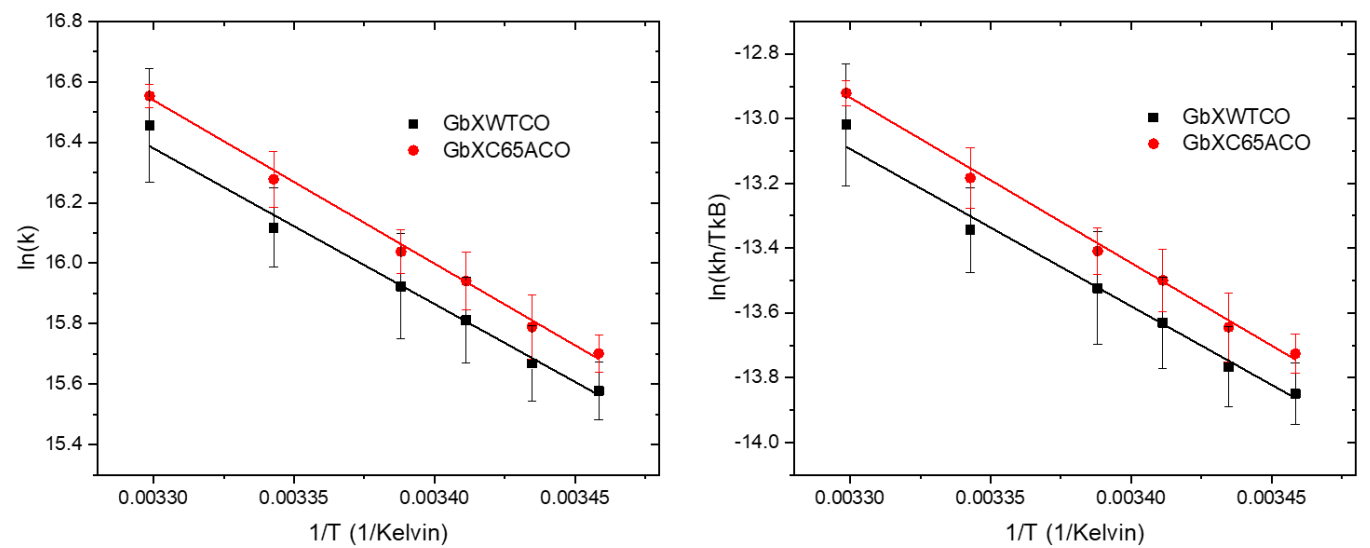

Figure 4.11. Arrhenius (left) and Eyring (right) plot of slow phase for CO photo-dissociation from GbXWT and C65A mutant.

the dissociation of $\mathrm{CO}$ from the distal pocket in $\mathrm{GbX}$ is more entropically favored. In addition, the 137 ns time constant determined for CO escape from GbXWT is similar to time constant observed for ligand escape from CygbWT ( $\tau \sim 150 \mathrm{~ns})$ and ligand escape 
from $\mathrm{Mb}(\tau \sim 150 \mathrm{~ns}$ at $\mathrm{pH} 3.5)$. The fast $\mathrm{CO}$ escape observed for $\mathrm{GbX}$ and $\mathrm{Cygb}$ can be associated with the increased dynamic of the distal histidine side-chain dynamics is reported to be controlled by Phe46, a residue that is conserved in the $\mathrm{Mb}$ and $\mathrm{Cygb}$ sequence.

\begin{tabular}{|c|c|c|c|c|c|c|c|c|}
\hline & Temperature $\left({ }^{\circ} \mathrm{C}\right)$ & $\begin{array}{c}\Delta \mathrm{H}_{1} \\
\left(\mathrm{kcal} \mathrm{mol}^{-1}\right)\end{array}$ & $\begin{array}{c}\Delta \mathrm{V}_{1} \\
\left(\mathrm{~mL} \mathrm{~mol}^{-1}\right)\end{array}$ & $\begin{array}{c}\Delta \mathrm{H}_{2} \\
\left(\mathrm{kcal} \mathrm{mol}^{-1}\right)\end{array}$ & $\begin{array}{c}\Delta \mathrm{V}_{2} \\
\left(\mathrm{~mL} \mathrm{~mol}^{-1}\right)\end{array}$ & $\begin{array}{c}\tau \\
(\mathbf{n s})\end{array}$ & $\begin{array}{c}\Delta \mathbf{H}^{\#} \\
\text { (kcal mol-1) }\end{array}$ & $\begin{array}{c}\Delta S^{\#} \\
\text { (cal mol-1 K-1) }\end{array}$ \\
\hline GbXWT-CO & $16-30$ & $28 \pm 9$ & $5 \pm 2$ & $1.4 \pm 9$ & $10 \pm 1.5$ & 137 & $10.1 \pm 1.4$ & $7.2 \pm 4.8$ \\
\hline GbXC65A-CO & $16-30$ & $13 \pm 4$ & $3.4 \pm 1.8$ & $2.4 \pm 2.8$ & $6.6 \pm 0.7$ & 120 & $10.0 \pm 0.6$ & $7.2 \pm 2.1$ \\
\hline${ }^{*}$ CygbWT-Co & $16-35$ & $1.1 \pm 3.6$ & $1.7 \pm 0.7$ & $7.5 \pm 2.5$ & $8.6 \pm 0.5$ & 150 & $9.2 \pm 0.4$ & $4.1 \pm 1.3$ \\
\hline${ }^{*} \mathrm{Mb}-\mathrm{CO}$ & $16-35$ & $7.4 \pm 2.0$ & $-1.7 \pm 0.5$ & $6.9 \pm 2.9$ & $12.1 \pm 0.7$ & 700 & $10.2 \pm 0.7$ & $4.0 \pm 2.2$ \\
\hline
\end{tabular}

Table 4.7. Reaction ( $\Delta \mathrm{H}$ and $\Delta \mathrm{V})$ and activation $\left(\Delta \mathrm{H}^{\#}\right.$ and $\left.\Delta \mathrm{V}^{\#}\right)$ parameters associated with the photo-dissociation of $\mathrm{Fe}-\mathrm{CO}$ bond and subsequent ligand escape from the protein matrix in GbX variants. (*(Astudillo et al., 2013))

Since this phenylalanine is also conserved in $\mathrm{GbX}$, it is possible it could also play a role in modulating the distal histidine orientations. The thermodynamic parameters for $\mathrm{CO}$ escape from GbX variants were determined by plotting $\mathrm{E}_{\mathrm{hv}}(\phi-1) / \Phi$ as a function of the temperature dependent parameter $(\mathrm{Cp} \rho / \beta)$, according to Eqs. 3.33 and 3.34 (Figure 4.11) and were listed in Tables 4.7 and 4.8.
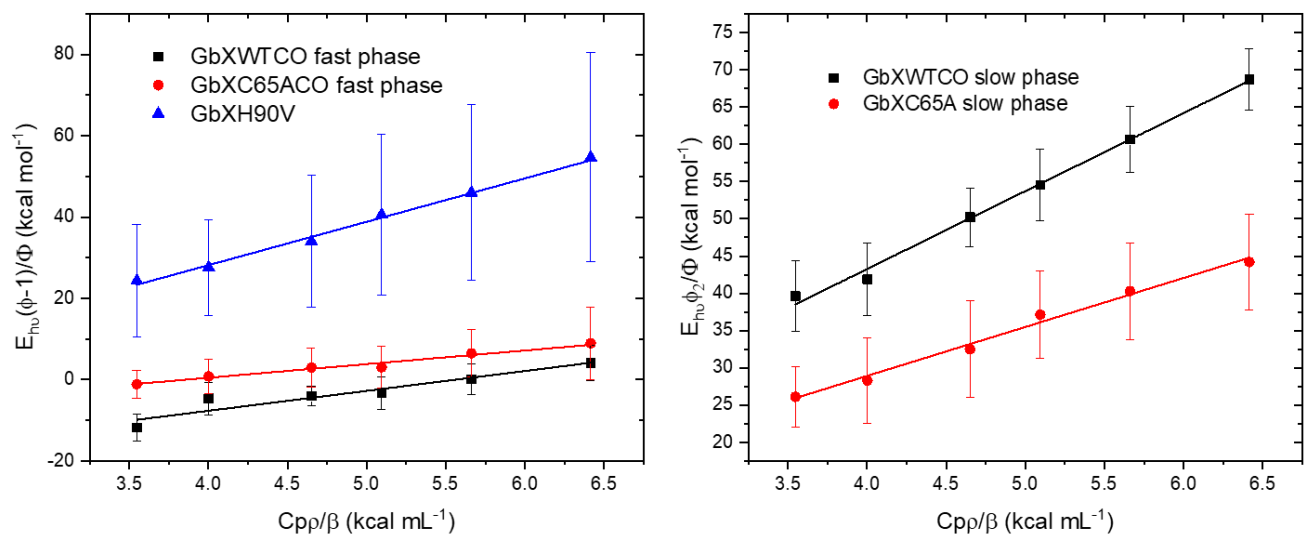

Figure 4.12. Plot of $\phi_{i} E_{h v}$ versus $C_{p} \rho / \beta$ for the prompt phase (left) and the slow phase (right) for $\mathrm{CO}$ photo-dissociation from $\mathrm{GbX}$ variants. 
The photo-cleavage of the Fe-CO bond and subsequent ligand release from GbXWT are associated with an enthalpy change of $\Delta \mathrm{H}_{1}=28 \pm 9 \mathrm{kcal} \mathrm{mol}^{-1}$ and a negligible volume change $\Delta \mathrm{V}_{1}=5 \pm 2 \mathrm{~mL} \mathrm{~mol}^{-1}$. The accompanying enthalpy change is reflecting the enthalpy of the cleavage of Fe-CO bond $\left(\Delta \mathrm{H}_{\mathrm{Fe}-\mathrm{CO}}=17 \mathrm{kcal} \mathrm{mol}^{-1}\right)$. The following ligand escape from the protein matrix is slightly endothermic $\left(\Delta \mathrm{H}_{2}=1.4 \pm 9 \mathrm{kcal} \mathrm{mol}^{-}\right.$ $\left.{ }^{1}\right)$ and leads to a small volume increase $\left(\Delta \mathrm{V}_{2}=10 \pm 1.5 \mathrm{~mL} \mathrm{~mol}^{-1}\right)$. The overall enthalpy changes and volume change observed for $\mathrm{CO}$ release from $\mathrm{GbX}$ variants as well as other previously studied globins are listed in Table 4.8. In order to have a better understanding of structural changes associated with the $\mathrm{CO}$ photo-dissociation, the measured reaction volume and enthalpy changes were used to estimate structural enthalpy change $\left(\Delta \mathrm{H}_{\text {str }}\right)$ and structure volume change $\left(\Delta \mathrm{V}_{\text {str }}\right)$ using Euation 3.47 and 3.48 .

$$
\begin{aligned}
& \Delta H_{\text {total }}=\Delta H_{F e-C O}+\Delta H_{s t r} \\
& \Delta V_{\text {total }}=\Delta V_{C O}^{\emptyset}+V_{5 c}^{\emptyset} G b X-V_{6 c}^{\emptyset} G b X C O
\end{aligned}
$$

Where $V_{C O}^{\emptyset}$ is the partial molar volume of CO $\left(37.3 \mathrm{~mL} \mathrm{~mol}^{-1}\right), \mathrm{V}_{s t r}=V_{5 c}^{\emptyset} G b X-$ $V_{6 c}^{\emptyset} G b X C O$ which describes the difference between the partial molar volume of the penta-coordinate GbX and CO-bound GbX (Moore et al., 1982). Considering the fluctuation of the values, the structure enthalpy changes of GbXWT $\left(\Delta \mathrm{H}_{\mathrm{str}}=12 \pm 13\right.$ $\left.\mathrm{kcal} \mathrm{mol}{ }^{-1}\right)$ is comparable in $\mathrm{GbXC65 \textrm {A }}\left(\Delta \mathrm{H}_{\mathrm{str}}=-2 \pm 5 \mathrm{kcal} \mathrm{mol}^{-1}\right)$ and GbXH90V $\left(\Delta \mathrm{H}_{\mathrm{str}}=-2 \pm 5 \mathrm{kcal} \mathrm{mol}^{-1}\right)$, imply the enthalpy change upon the dissociation of $\mathrm{CO}$ is mainly the result of iron $\mathrm{CO}$ bond breakage. On the other hand, the structure volume 


\begin{tabular}{|c|c|c|c|c|c|c|}
\hline & $\begin{array}{c}\text { Temperature } \\
\left({ }^{\circ} \mathrm{C}\right)\end{array}$ & $\begin{array}{c}\Delta \mathbf{H}_{\text {total }} \\
\left(\mathrm{kcal} \mathrm{mol}^{-1}\right)\end{array}$ & $\begin{array}{c}\Delta \mathbf{H}_{\mathrm{str}} \\
\left(\mathrm{kcal} \mathrm{mol}^{-1}\right)\end{array}$ & $\underset{\left(\mathrm{mL} \mathrm{mol}^{-1}\right)}{\Delta \mathrm{V}_{\text {total }}}$ & $\underset{\left(\mathrm{mL} \mathbf{~ m o l}^{-1}\right)}{\Delta V_{\text {trr }}}$ & $\begin{array}{c}\Delta \mathrm{V}_{\mathrm{str}+\mathrm{H}_{2} \mathrm{O}} \\
\left(\mathrm{mL} \mathrm{mol}^{-1}\right)\end{array}$ \\
\hline GbXWT-CO & $16-30$ & $29 \pm 13$ & $12 \pm 13$ & $15 \pm 2.5$ & $-22.3 \pm 2.5$ & $-2.3 \pm 2.5$ \\
\hline GbXC65A-CO & $16-30$ & $15 \pm 5$ & $-2 \pm 5$ & $10 \pm 2$ & $-27.3 \pm 2$ & $-9.3 \pm 2$ \\
\hline GbXH90V-CO & $16-30$ & $15 \pm 5$ & $-2 \pm 5$ & $11 \pm 5$ & $-26.3 \pm 2$ & $-8.3 \pm 2$ \\
\hline${ }^{*}$ CygbWT-CO & $16-35$ & $9 \pm 4$ & $-8 \pm 4$ & $10.3 \pm 1.2$ & $-27 \pm 1.2$ & $-9 \pm 1.2$ \\
\hline *NgbWT-CO & $16-35$ & $20 \pm 4$ & $3 \pm 4$ & $13.4 \pm 0.9$ & $-23.9 \pm 0.9$ & $-5.9 \pm 0.9$ \\
\hline${ }^{*} \mathrm{Mb}-\mathrm{CO}$ & $16-35$ & $14 \pm 3$ & $-3 \pm 3$ & $10.4 \pm 0.7$ & $-26.9 \pm 0.7$ & $-8.9 \pm 0.7$ \\
\hline${ }^{\dagger} \mathrm{Hb}-\mathrm{CO}$ & $16-35$ & $18 \pm 2.9$ & $1 \pm 2.9$ & $23.4 \pm 0.5$ & $-13.9 \pm 0.9$ & $4.1 \pm 0.9$ \\
\hline
\end{tabular}

Table 4.8. Total volume and enthalpy changes associated with the $\mathrm{CO}$ dissociation from $\mathrm{GbX}$ variants as well as Cygb, Ngb, Mb, and Hb. (*(Astudillo et al., 2013), †(Peters et al., 1992)) change in GbXWT $\left(\Delta \mathrm{V}_{\text {str }}=-22.3 \pm 2.5 \mathrm{~mL} \mathrm{~mol}^{-1}\right)$ is slightly less than both GbXC65A $\left(\Delta \mathrm{V}_{\mathrm{str}}=-27.3 \pm 2 \mathrm{~mL} \mathrm{~mol}^{-1}\right)$ and $\mathrm{GbXH} 90 \mathrm{~V}\left(\Delta \mathrm{V}_{\mathrm{str}}=-26.3 \pm 2 \mathrm{~mL} \mathrm{~mol}^{-1}\right)$. It was reported that water molecules could enter the distal pocket of $\mathrm{Mb}$ when exogenous ligand is not present. It is possible that after the dissociation of $\mathrm{CO}$ from the $\mathrm{GbX}$, a water molecule could enter the distal pocket. Taking into account the water molecule, the $\Delta \mathrm{V}_{\mathrm{str}+\mathrm{H} 2 \mathrm{O}}$ of GbXWT, GbXC65A and GbXH90V is $-2.3 \pm 2.5 \mathrm{~mL} \mathrm{~mol}^{-1},-9.3 \pm 2 \mathrm{~mL} \mathrm{~mol}^{-1}$, and -8.3 $\pm 2 \mathrm{~mL} \mathrm{~mol}{ }^{-1}$, respectively. Therefore, the overall structure of GbXWT remain unchanged upon the dissociation of $\mathrm{CO}$ while there is small contraction of the protein conformation in both GbXC65A and GbXH90V. Interestingly, while shown a similar structure enthalpy change to other globins, the negligible structure volume change observed for CO release from GbXWT is distinct from other globins studied using PAC indicating distinct structural changes upon $\mathrm{CO}$ dissociation. This is also consistent with distinct activation entropy changes observed for $\mathrm{CO}$ binding to $\mathrm{GbX}$ variants. 


\subsection{Discussion}

The results presented here demonstrate several distinct properties of GbX. Interestingly, this protein demonstrates a high stability towards $\mathrm{pH}$ as the $\mathrm{pH}_{\text {mid }}$ value for the $\mathrm{pH}$ induced unfolding of GbXWT is significantly lower than that observed previously for pentacoordinate $\mathrm{hhMb}$ as well as hexacoordinate Ngb suggesting an increased stability of this protein. Interestingly, the increased stability cannot be fully attributed to coordination bond between the distal histidine and heme iron as GbXH90V construct exhibits increased $\mathrm{pH}$ stability with respect to $\mathrm{Mb}$. This suggest more stronger interaction between the proximal histidine and heme iron in GbX.

Affinity constants for $\mathrm{CN}^{-}$binding to $\mathrm{GbX}$ variants are significantly lower than that observed for other hexa-coordinate globins, pointing towards lower reactivity of iron atom in $\mathrm{GbX}$ towards this ligand. Interestingly, two affinity constants determined

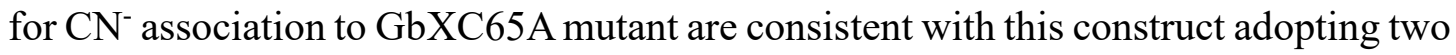
conformations in the met form. The low cyanide affinity conformation in the cysteine mutant is similar to WT while the high cyanide affinity conformation binds $\mathrm{CN}^{-}$with the similar affinity as the GbXH90V mutant, suggesting that in the high affinity conformation, the distal histidine is weakly associated to the heme iron. Previous studies shown that the disulfide bridge in Cygb modulates its ligand affinity and the disruption of the disulfide bridge leads to the rearrangement of the E helix where distal histidine is located and ultimately change the position of distal histidine. Also, crystallographic study of Cygb structure revealed the existence of two different 
conformations of the protein with two distinct orientations of the distal histidine. Analogously, the disruption of the disulfide bridge in GbX may lead to two different protein conformations with distinct position of distal histidine that leads to two different affinities for $\mathrm{CN}^{-}$binding.

In addition, the $\mathrm{CO}$ binding to $\mathrm{GbX}$ is heterogenous, with 4 different rate constants resolved by transient absorption spectroscopy. In general, the removal of the disulfide bond decreases the rate constant for $\mathrm{CO}$ rebinding three times with respect to GbXWT, whereas the replacement of distal histidine increases the $\mathrm{CO}$ rebinding rate eight times. More importantly, the kinetics are significantly slower compare to the CO rebinding kinetics to Cygb. The fast rate constants for $\mathrm{CO}$ binding to $\mathrm{Cygb}$ were associated with a highly reactive heme iron in this protein. The slow rebinding observed for GbX is consistent with the heme iron reactivity being similar to $\mathrm{Mb}$ or rice $\mathrm{Hb}$. Besides, the $\mathrm{CO}$ concentration dependent experiment show a high dissociation constant of $\mathrm{CO}$ and a small affinity constant for distal histidine binding to $\mathrm{GbX}$ heme iron, further supporting the hypothesis that the heme iron inside $\mathrm{GbX}$ is less reactive compare to other vertebrate globins.

The quantum yield for bimolecular $\mathrm{CO}$ rebinding to $\mathrm{GbX}$ variants reveal that the disruption of internal disulfide bridge promotes the $\mathrm{CO}$ to escape from the protein by increasing the energy barrier for the geminate $\mathrm{CO}$ rebinding whereas the removal of distal histidine facilitates the $\mathrm{CO}$ geminate rebinding. Photo-acoustic calorimetry data shows that the $\mathrm{CO}$ escape from GbXWT and GbXC65A is a two-step process with a 
time constant around $130 \mathrm{~ns}$ which is similar to time constant measured for $\mathrm{CO}$ escape from $\mathrm{Cygb}$ and $\mathrm{Mb}$ at $\mathrm{pH}$ 3.5. The activation barrier for $\mathrm{CO}$ dissociation in $\mathrm{GbX}$ is similar to $\mathrm{Cygb}$ and $\mathrm{hhMb}$ but with a higher activation entropy. In addition, the structure enthalpy change for $\mathrm{CO}$ escape from $\mathrm{GbX}$ is similar to other globins while the structural volume change is less than that measured in other globins studied by PAC. These results imply a distinct structural change accompanying $\mathrm{CO}$ dissociation from the GbX protein matrix. 


\section{CHARACTERIZATION OF THE CONFORMATION, REGULATION, ORIENTATION, AND HEME ACCESIBILITY IN HEXACOORDINATE GLOBINS BY USING FLUORESCENT HEME ANALOG.}

\subsection{Introduction}

The development of a method to extract the heme prosthetic group from globin proteins is of paramount importance since it allows to investigate the interaction between the heme group and the surrounding amino acid residues in the heme cavity, as well as how these interactions affect or regulate the function of the heme protein, using NMR spectroscopy and fluorescence techniques (Fanelli et al., 1958; Teale, 1959). The native holoMb and the reconstituted FePPIX-Mb (heme group extracted from the native protein and reconstituted with the synthesized iron protoporphyrin IX) present no differences in terms of spectra properties, confirming the reversibility of the recombination reaction (HARRISON \& BLOUT, 1965). Other than iron protoporphyrin IX, other porphyrin-based molecules can be used to replace the heme prosthetic group inside the heme protein. By using heme analogs, the role of the peripheral side chains of the heme group can be examined to determine their impact on structure-function relation between the heme and globin scaffold. For instance, heme7-propionate affects binding between the proximal histidine and heme iron in horse heart myoglobin, while removing heme-6-propionate can disrupt hydrogen bonding 
network in the distal pocket which impacts the autoxidation of the heme (Hayashi et al., 2002).

Furthermore, fluorescent heme analogs such as free based porphyrin or zinc protoporphyrin IX (ZnPPIX) are also used to study structural function relationship of the proteins by applying fluorescence techniques. Due to the iron coordination to the porphyrin ring, the iron heme is non-fluorescent. In addition, due to a significant spectral overlap between the emission spectrum of tryptophan and absorption spectrum of heme in the native state, the iron in the porphyrin ring efficiently quenches the intrinsic tryptophan fluorescence (Weber \& Teale, 1959). Thus, replacement of the native heme with its fluorescent analogs allows fluorescence- and phosphorescencebased characterizations of structural and dynamic properties of heme proteins. ZnPPIX reconstituted hemoglobin were used to investigate the allosteric effects, while ZnPPIX reconstituted $\mathrm{Mb}$ were used to study its interactions with cytochrome b5 (Liang et al., 2002; Naito et al., 1998).

Quenching ZnPPIX reconstituted globins with small molecules provides a way to measure how small molecules diffuse through the protein matrix without the process being influenced by the actual binding step (Barboy \& Feitelson, 1987). By introducing a chemical modification or mutation of the protein, specific amino acid residues or structural features can be examined and their impact on the ligand diffusion or migration pattern in the proteins can be determined (Aono et al., 1995). In addition, ZnPPIX has a longer triplet state lifetime than other metallo-porphyrins and $\mathrm{O}_{2}$ is well 
known as a highly efficient quencher of excited triplet states (Aono et al., 1995). Therefore, triplet state quenching studies of ZnPPIX reconstituted globins allow to study the mechanism of $\mathrm{O}_{2}$ diffusion and migration into the heme bind site. This is particularly important since $\mathrm{O}_{2}$ is an important physiological molecule (Barboy \& Feitelson, 1987).

It was previously shown by our group that hexa-coordinate globins can be successfully reconstituted by ZnPPIX and the incorporated fluorescent analogue exhibits fluorescent properties that are distinct from those observed previously for penta-coordinate proteins, $\mathrm{Mb}$ and $\mathrm{Hb}$ (Tangar et al., 2019). Here we report reconstitution of several variants of Ngb and Cygb, with several ZnPPIX to investigate how conformational changes in terms of removal the distal histidine coordination and the absence of the internal disulfide bond affect the heme orientation and ligand diffusion into the heme cavity.

\subsection{Results}

\subsubsection{Steady-state UV-vis absorption spectroscopy}

Steady-state UV-vis absorption spectra of reconstituted ZnPPIXCygb, ZnPPIX-

$\mathrm{Ngb}$ as well as their mutants are shown in Figure 5.1. Incorporation of ZnPPIX into wild type Cygb results in a Soret band maximum at $428 \mathrm{~nm}, \beta$ band at $553 \mathrm{~nm}$ and a split $\alpha$ band situated at $587 \mathrm{~nm}$ and $595 \mathrm{~nm}$. All Cygb mutants showed a comparable absorption spectrum with a similar location of $\lambda_{\max }$ of the Soret band and $\beta$ band. Interestingly, the absorption spectrum for ZnPPIX-CygbC38S and ZnPPIX- 

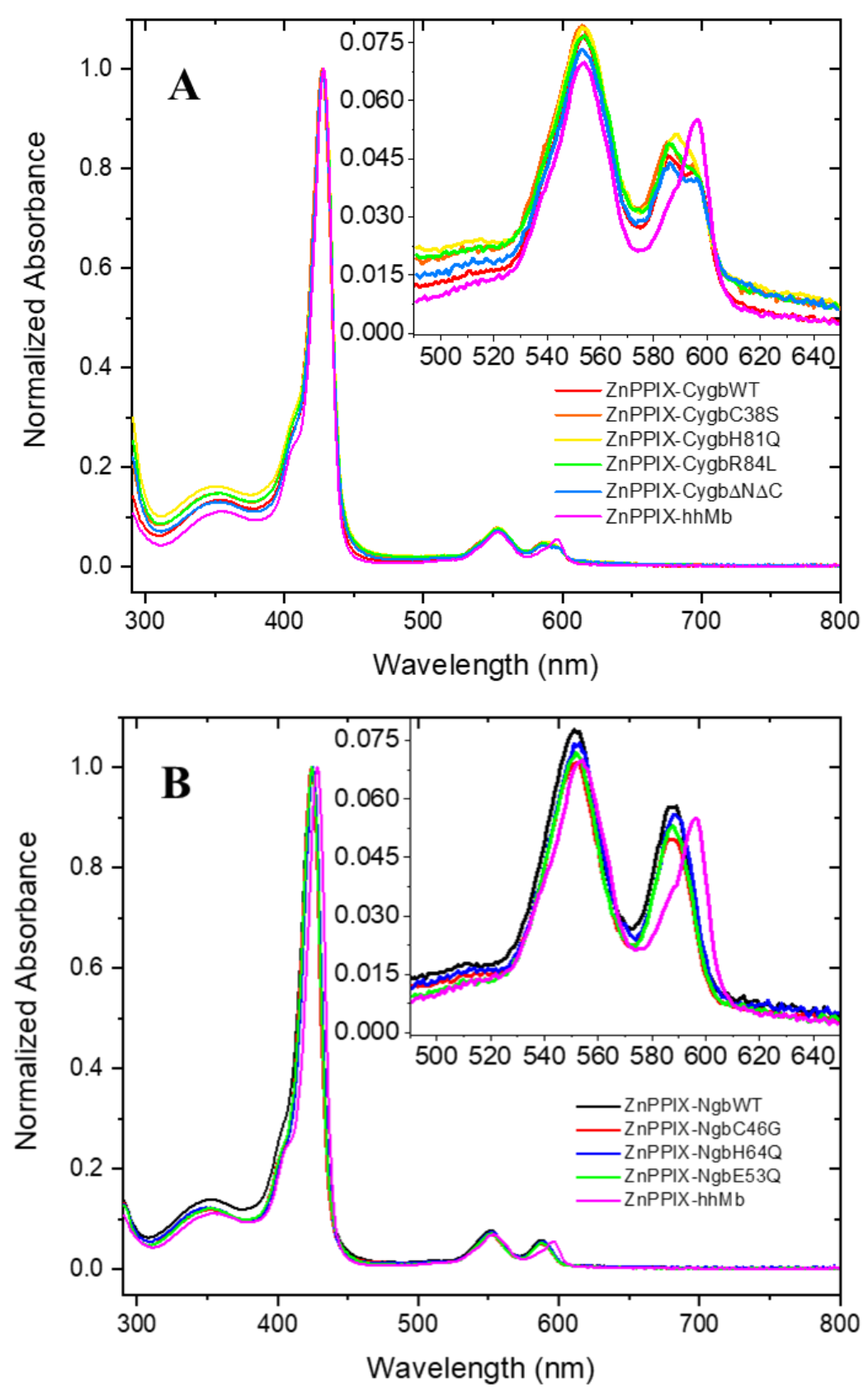

Figure 5.1. Normalized steady-state absorption spectra of ZnPPIX-hhMb and ZnPPIX-Cygb variants (A) and $\mathrm{ZnPPIX-Ngb} \mathrm{variants} \mathrm{(B)} \mathrm{in} 50 \mathrm{mM}$ TrisHCl pH 7.0.

$\operatorname{Cygb} \Delta \mathrm{N} \Delta \mathrm{C}$ showed similar $\alpha$ band split as observed in the absorption spectrum of the CygbWT. In the absorption spectrum of ZnPPIX-CygbR84L, the peak located at 595 $\mathrm{nm}$ has a lower intensity than the peak at $586 \mathrm{~nm}$. Notably, the absorption spectrum for the ZnPPIX-CygbH81Q mutant exhibited a single $\alpha$-band that is broader that an $\alpha$-band observed in ZnPPIX-Mb. On the other hand, the absorption spectrum of reconstituted 


\begin{tabular}{|c|c|c|c|}
\hline & Soret & $\boldsymbol{\beta}$ & $\alpha$ \\
\hline ZnPPIX-hhMb & $428 \mathrm{~nm}$ & $554 \mathrm{~nm}$ & $595 \mathrm{~nm}$ \\
\hline ZnPPIX-CygbWT & $428 \mathrm{~nm}$ & $553 \mathrm{~nm}$ & $585 / 594 \mathrm{~nm}$ \\
\hline ZnPPIX-CygbC38S & $427 \mathrm{~nm}$ & $553 \mathrm{~nm}$ & $585 / 595 \mathrm{~nm}$ \\
\hline ZnPPIX-CygbH81Q & $428 \mathrm{~nm}$ & $553 \mathrm{~nm}$ & $588 \mathrm{~nm}$ \\
\hline ZnPPIX-CygbR84L & $428 \mathrm{~nm}$ & $554 \mathrm{~nm}$ & $586 * \mathrm{~nm}$ \\
\hline ZnPPIX-Cygb $\Delta N \Delta C$ & $428 \mathrm{~nm}$ & $553 \mathrm{~nm}$ & $586 / 596 \mathrm{~nm}$ \\
\hline ZnPPIX-NgbWT & $425 \mathrm{~nm}$ & $552 \mathrm{~nm}$ & $588 \mathrm{~nm}$ \\
\hline ZnPPIX-NgbC46G & $424 \mathrm{~nm}$ & $552 \mathrm{~nm}$ & $587 \mathrm{~nm}$ \\
\hline ZnPPIX-NgbH64Q & $425 \mathrm{~nm}$ & $553 \mathrm{~nm}$ & $588 \mathrm{~nm}$ \\
\hline ZnPPIX-NgbE53Q & $424 \mathrm{~nm}$ & $552 \mathrm{~nm}$ & $588 \mathrm{~nm}$ \\
\hline
\end{tabular}

Table 5.1. Summary of UV-vis absorption maxima for ZnPPIX reconstituted hhMb, Cygb and $\mathrm{Ngb}$ variants. Specifically, CygbR84L shown a shoulder rather than a peak at $\sim 595 \mathrm{~nm}$.

NgbWT showed a Soret band at $425 \mathrm{~nm}$, with $\beta$ and $\alpha$ bands centered at 552 and 588 nm, respectively. All Ngb mutants showed identical position of the Soret band as well as $\beta$ and $\alpha$ bands to the wild type protein. Reconstituted ZnPPIX-hhMb was also prepared and characterized as a control and its absorption spectrum was characterized by a Soret band at $428 \mathrm{~nm}$, with $\beta$ and $\alpha$ bands centered at 554 and $595 \mathrm{~nm}$ which are comparable to the previously published data (ALBANI \& ALPERT, 1987).

\subsubsection{Steady-state fluorescence emission spectra}

Fluorescent emission spectra revealed that, upon excitation at $421 \mathrm{~nm}$, steady-state emission of ZnPPIX reconstituted wild type Cygb exhibits a narrow peak at $598 \mathrm{~nm}$ and a broad band of weaker intensity at $649 \mathrm{~nm}$ (figure 5.2). Emission spectra for Cygb mutants showed a comparable $\lambda_{\max }$ for both peaks, but the peaks of shorter wavelength in ZnPPIX-CygbH81Q and ZnPPIX-Cygb $\Delta \mathrm{N} \Delta \mathrm{C}$ are $\sim 1 \mathrm{~nm}$ blue shifted compare to wild type Cygb. On the other hand, the emission spectrum of ZnPPIX-NgbWT is blueshifted, with the one peak situated at $593 \mathrm{~nm}$ and the longer wavelength peak located 
at $647 \mathrm{~nm}$. Emission spectra for Ngb mutants show a similar $\lambda_{\max }$ for both emission peaks with exception of the emission spectrum for ZnPPIX-NgbC46G mutant that has the $\lambda_{\max }$ of the longer emission band $2 \mathrm{~nm}$ blue shifted compared to the ZnPPIXNgbWT.
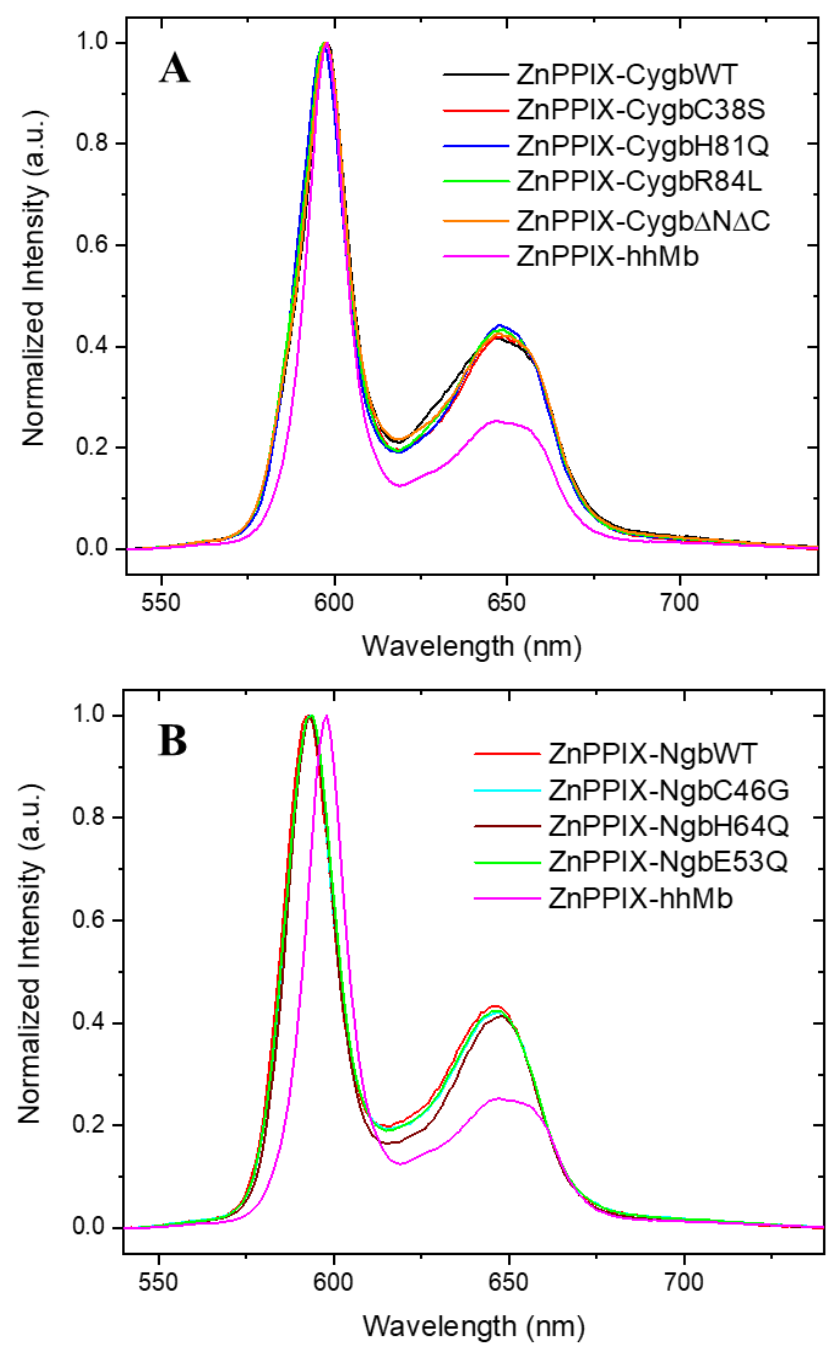

Figure 5.1. Normalized steady-state fluorescence emission of ZnPPIX-Cygb variants (A) and ZnPPIX-Ngb variants (B), using $\lambda_{\text {exc }}=421 \mathrm{~nm}$.

Notably, ZnPPIX-NgbH64Q $648 \mathrm{~nm}$ emission band is narrower compared to the ZnPPIX-NgbWT, pointing towards a less heterogenous surrounding of the fluorophore environment compared to all the mutants. 


\begin{tabular}{|l|c|c|}
\hline & $\lambda_{\mathbf{m a x}_{\mathbf{1}}}$ & $\boldsymbol{\lambda}_{\mathbf{m a x}}$ \\
\hline ZnPPIX-hhMb & $598 \mathrm{~nm}$ & $650 \mathrm{~nm}$ \\
\hline ZnPPIX-CygbWT & $598 \mathrm{~nm}$ & $649 \mathrm{~nm}$ \\
\hline ZnPPIX-CygbC38S & $598 \mathrm{~nm}$ & $649 \mathrm{~nm}$ \\
\hline ZnPPIX-CygbH81Q & $597 \mathrm{~nm}$ & $648 \mathrm{~nm}$ \\
\hline ZnPPIX-CygbR84L & $597 \mathrm{~nm}$ & $649 \mathrm{~nm}$ \\
\hline ZnPPIX-Cygb $\Delta N \Delta C$ & $598 \mathrm{~nm}$ & $648 \mathrm{~nm}$ \\
\hline ZnPPIX-NgbWT & $593 \mathrm{~nm}$ & $647 \mathrm{~nm}$ \\
\hline ZnPPIX-NgbC46G & $593 \mathrm{~nm}$ & $645 \mathrm{~nm}$ \\
\hline ZnPPIX-NgbH64Q & $593 \mathrm{~nm}$ & $648 \mathrm{~nm}$ \\
\hline ZnPPIX-NgbE53Q & $594 \mathrm{~nm}$ & $646 \mathrm{~nm}$ \\
\hline
\end{tabular}

Table 5.2. Summary of fluorescence emission maxima for ZnPPIX reconstituted hhMb, Cygb and $\mathrm{Ngb}$ variants.

\subsubsection{Fluorescence and phosphorescence lifetime}

The single state lifetime of ZnPPIX reconstituted proteins was determined in the frequency mode and the plots of phase shift/modulation ratio as a function of

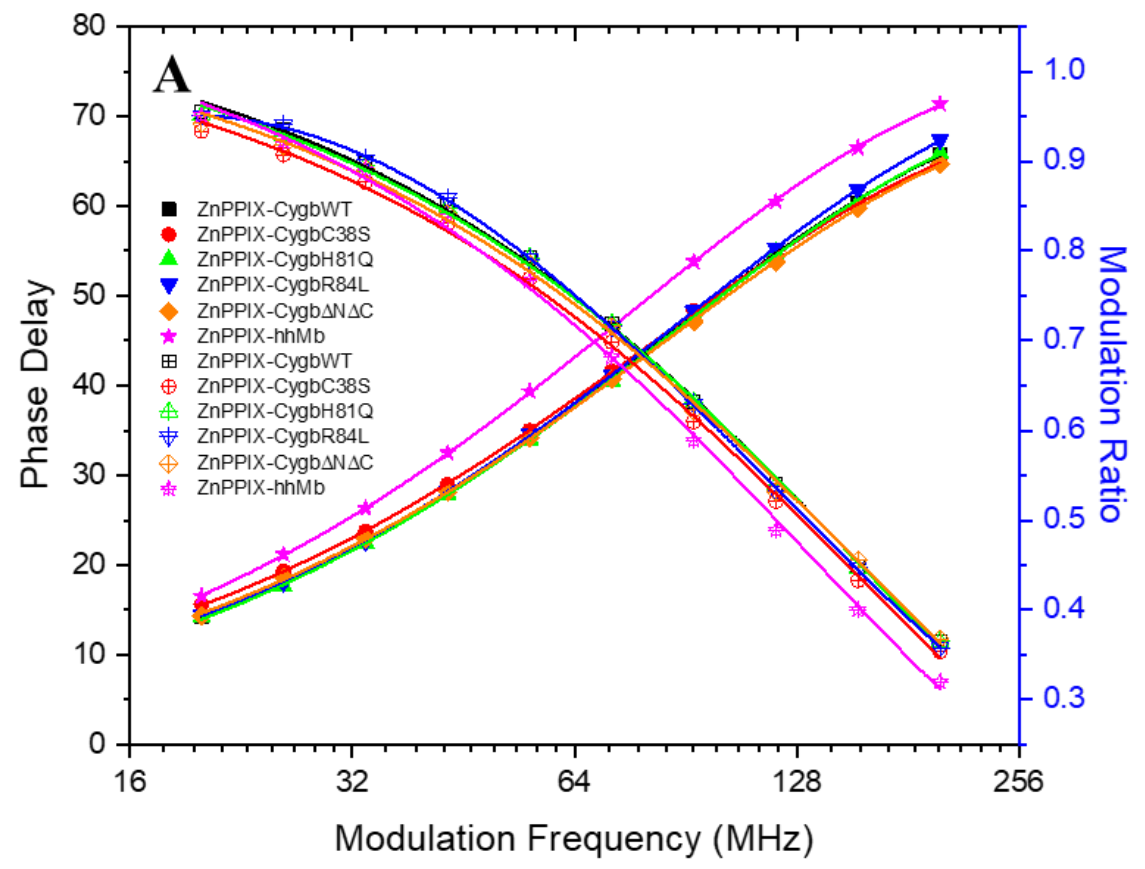




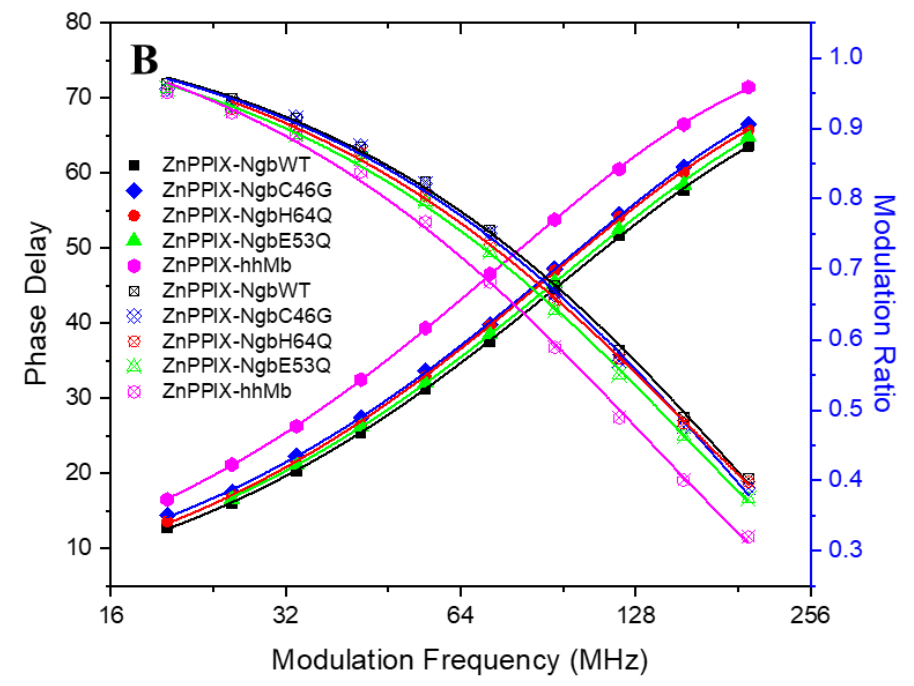

Figure 5.3. Time-resolved fluorescence in the frequency domain data determined for ZnPPIXCygb variants (A) and ZnPPIX-Ngb variants (B).

modulation frequency is shown in Figure 5.3. The data for ZnPPIX reconstituted Mb were analyzed using a single exponential decay model, whereas the results for the ZnPPIX reconstituted $\mathrm{Ngb}$ and Cygb constructs were analyzed using a sum of two exponential decays and the recovered decay parameters are listed in Table 5.3.

\begin{tabular}{|c|c|c|c|c|c|c|c|c|c|c|}
\hline & $\begin{array}{c}\text { ZnPPIX- } \\
\text { hhMb }\end{array}$ & $\begin{array}{l}\text { ZnPPIX- } \\
\text { CygbWT }\end{array}$ & $\begin{array}{c}\text { ZnPPIX- } \\
\text { CygbC38S }\end{array}$ & $\begin{array}{c}\text { ZnPPIX- } \\
\text { CygbH81Q }\end{array}$ & $\begin{array}{c}\text { ZnPPIX- } \\
\text { CygbR84L }\end{array}$ & $\begin{array}{c}\mathrm{ZnPPIX}- \\
\operatorname{Cygb} \Delta \mathbf{N} \Delta \mathrm{C}\end{array}$ & $\begin{array}{c}\text { ZnPPIX- } \\
\text { NgbWT }\end{array}$ & $\begin{array}{c}\mathrm{ZnPPIX}- \\
\text { NgbC46G }\end{array}$ & $\begin{array}{c}\text { ZnPPIX- } \\
\text { NgbH64Q }\end{array}$ & $\begin{array}{c}\text { ZnPPIX- } \\
\text { NgbE53Q }\end{array}$ \\
\hline$\tau_{1}(\mathrm{~ns})$ & $\mathrm{N} / \mathrm{A}$ & 0.787 & 1.02 & 0.785 & 1.61 & 1.55 & 0.959 & 1.76 & 0.738 & 1.45 \\
\hline$\alpha_{1}$ & N/A & 0.19 & 0.38 & 0.17 & 0.6 & 0.71 & 0.28 & 0.95 & 0.09 & 0.71 \\
\hline$\tau_{2}(\mathrm{~ns})$ & 2.36 & 2.12 & 2.43 & 2.07 & 2.46 & 2.86 & 1.94 & 4.22 & 1.92 & 2.49 \\
\hline$\alpha_{2}$ & 1 & 0.81 & 0.62 & 0.83 & 0.4 & 0.29 & 0.72 & 0.05 & 0.91 & 0.29 \\
\hline$\tau_{\mathbf{P}}(\mathrm{ms})$ & 15.5 & 14.8 & 11.2 & 13.7 & 13.1 & n.d. & 11.1 & 17.3 & 15.5 & n.d. \\
\hline
\end{tabular}

Table 5.3. Summary of fluorescence and phosphorescence parameters of ZnPPIX-reconstituted hexacoordinate globins.

The bimodal decay observed for ZnPPIX-Cygb WT and ZnPPIX-NgbWT exhibits two distinct fluorescence lifetimes with $\tau_{1} \sim 0.8 \mathrm{~ns}$ and $\tau_{2} \sim 2.1 \mathrm{~ns}$, and the preexponential factor associated with the faster decay $\alpha_{1}$, being $\sim 0.35$ and the preexponential factor associated with the longer lifetime, $\alpha_{2}$, being $\sim 0.65$. ZnPPIXCygbHis81Q mutant exhibits analogous lifetimes and associated pre-exponential 
factors as ZnPPIX-Cygb WT, whereas Cygb other mutants showed an increase in both lifetimes as well as increase of the $\alpha_{1}$ pre-exponential factor. ZnPPIX-NgbWT also exhibits a bimodal fluorescence decay with the lifetimes: $\tau_{1} \sim 1 \mathrm{~ns}$ and $\tau_{2} \sim 2 \mathrm{~ns}$, and the pre-exponential factors $\alpha_{1}=0.28$ and $\alpha_{2}=0.72$. Compared to ZnPPIX-NgbWT, ZnPPIXNgbH64G exhibits a shorter $\tau_{1}=0.74 \mathrm{~ns}$ and a smaller $\alpha_{1}$ value of 0.1 In case of ZnPPIX-NgbC46G and ZnPPIX-NgbE53Q mutants, $\tau_{1}$ increases to 1.76 ns and 1.45 ns, respectively, and the pre-exponential factor $\alpha_{1}$ is 0.95 and 0.75 , respectively. Interestingly, both residues, Cys 46 and Glu 53 are located in the CD loop of the protein, indicating that changes in this flexible loop impact the heme binding pocket. The second lifetime observed for ZnPPIXC46G is two times slower than that observed for the ZnPPIX-NgbWT, however the pre-exponential factor associated with this lifetime is small, $5 \%$.

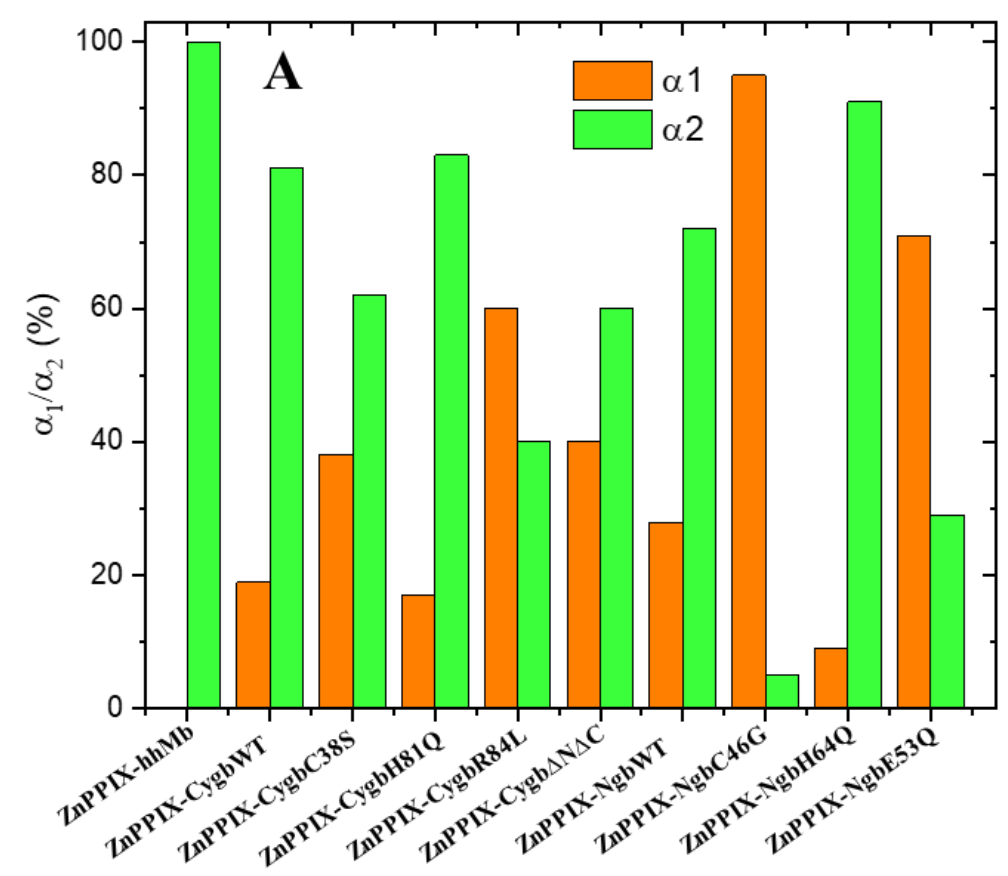




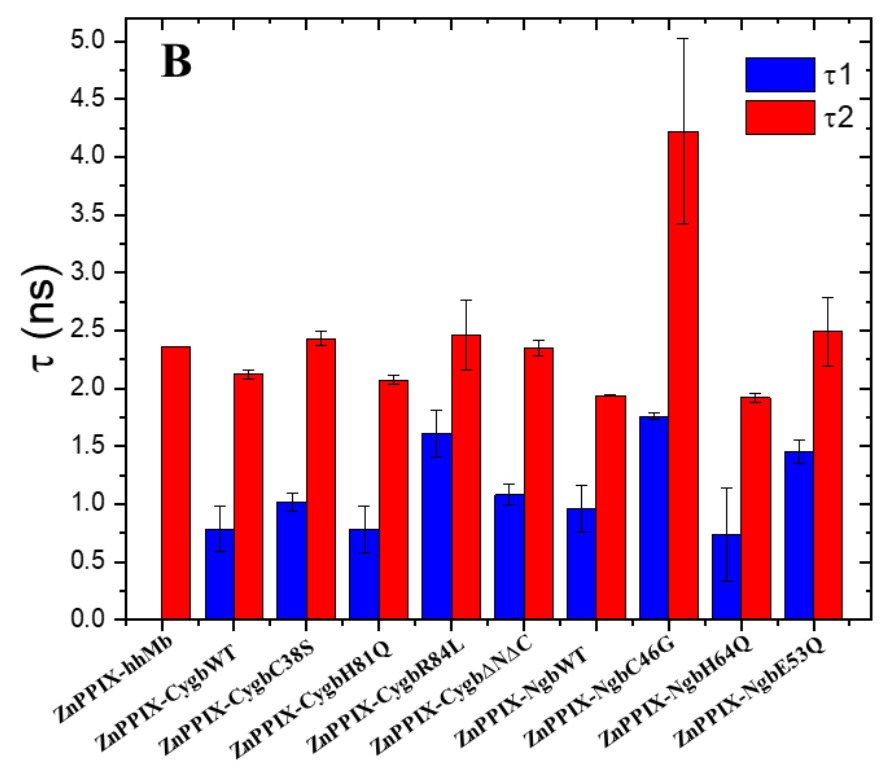

Figure 5.4. Time-resolved fluorescence in the frequency domain data determined for ZnPPIXCygb variants (A) and ZnPPIX-Ngb variants (B).

In addition to the characterization of the singlet lifetime of ZnPPIX, we have measured ZnPPIX triplet state lifetime by monitoring the time profile of the absorbance change of the triplet state at $447 \mathrm{~nm}$. The transient absorption traces are shown in Figure 5.5 and the data were analyzed using a single exponential decay model.

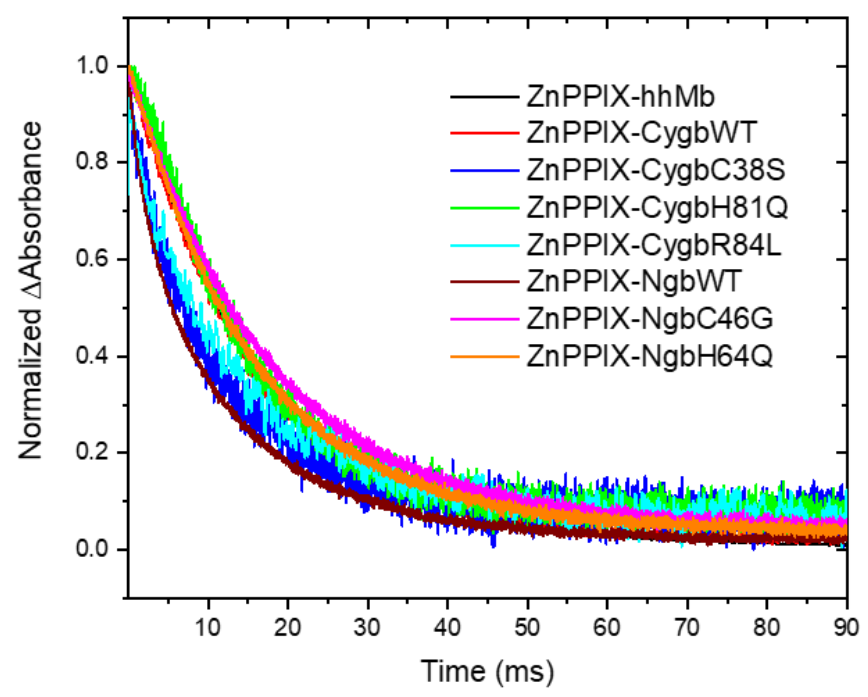

Figure 5.5. Phosphorescence decay determined for reconstituted globins. Phosphorescence decay was monitored at $447 \mathrm{~nm}$. 
Phosphorescence lifetime, $\tau_{\mathrm{p}}$, observed for ZnPPIX-Cygb variants varies from 11.2 $\mathrm{ms}$ to $14.8 \mathrm{~ms}$ and similar variations were observed for the triplet state lifetime of ZnPPIX-Ngb variants (from $11.1 \mathrm{~ms}$ to $17.3 \mathrm{~ms}$ ). The detected lifetimes are similar to the triplet state decay of ZnPPIX-hhMb, $\tau_{\mathrm{p}}=15.5 \mathrm{~ms}$. As the ZnPPIX lifetime is strongly impacted by the presence of oxygen in the deoxygenated proteins samples, it is possible that the observed deviations in the phosphorescence lifetime reflect the presence of the residual oxygen.

\subsubsection{Quenching study}

Methyl viologen $\left(\mathrm{MV}^{2+}\right)$ has been employed previously as an efficient quencher of the singlet state emission of ZnPPIX. To characterize interactions between $\mathrm{MV}^{2+}$ and ZnPPIX reconstituted proteins, we have monitored absorption spectra of ZnPPIX reconstituted proteins in the presence of increasing concentration of $\mathrm{MV}^{2+}$.

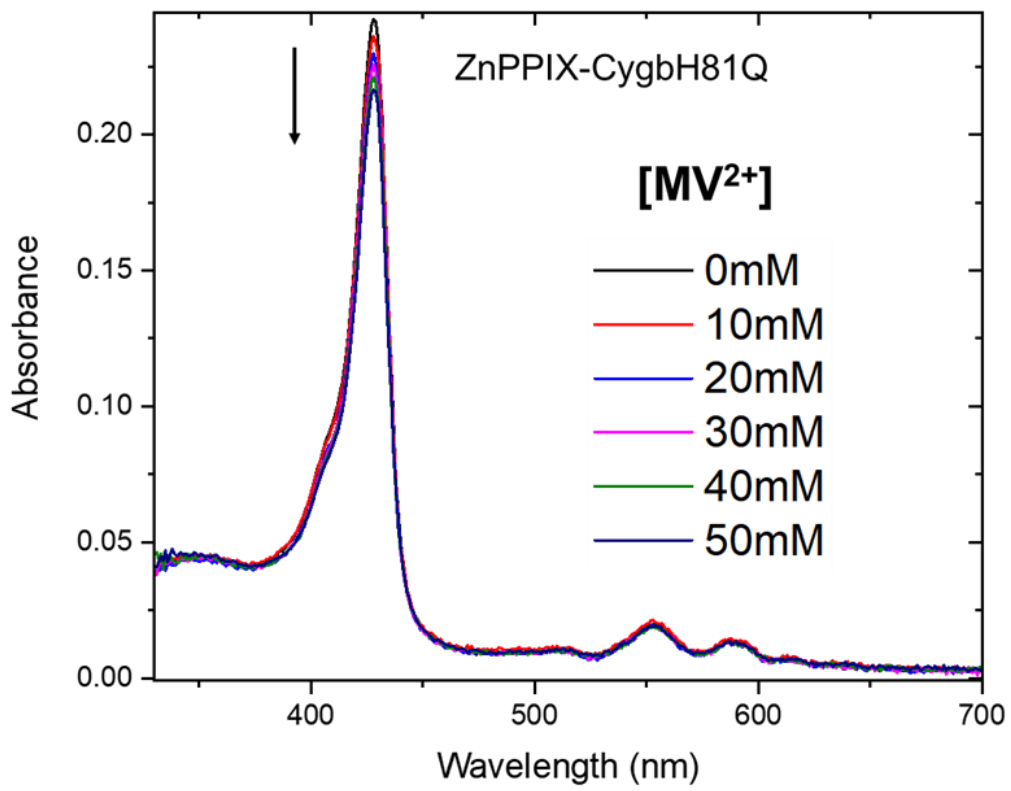

Figure 5.6. Example of UV-vis absorption spectra of ZnPPIX reconstituted protein with increasing concentration of methyl viologen. A decrease of Soret band absorbance is observed. 
In the presence of the quencher, the absorbance of the Soret band decreases, indicating $\mathrm{MV}^{2+}$ binding to the protein, possible in the vicinity of the heme binding pocket. The impact of $\mathrm{MV}^{2+}$ association to ZnPPIX-CygbH81Q mutant on ZnPPIX absorption spectrum is demonstrated in Figure 5.6 and similar results were obtained for other constructs, data not shown.

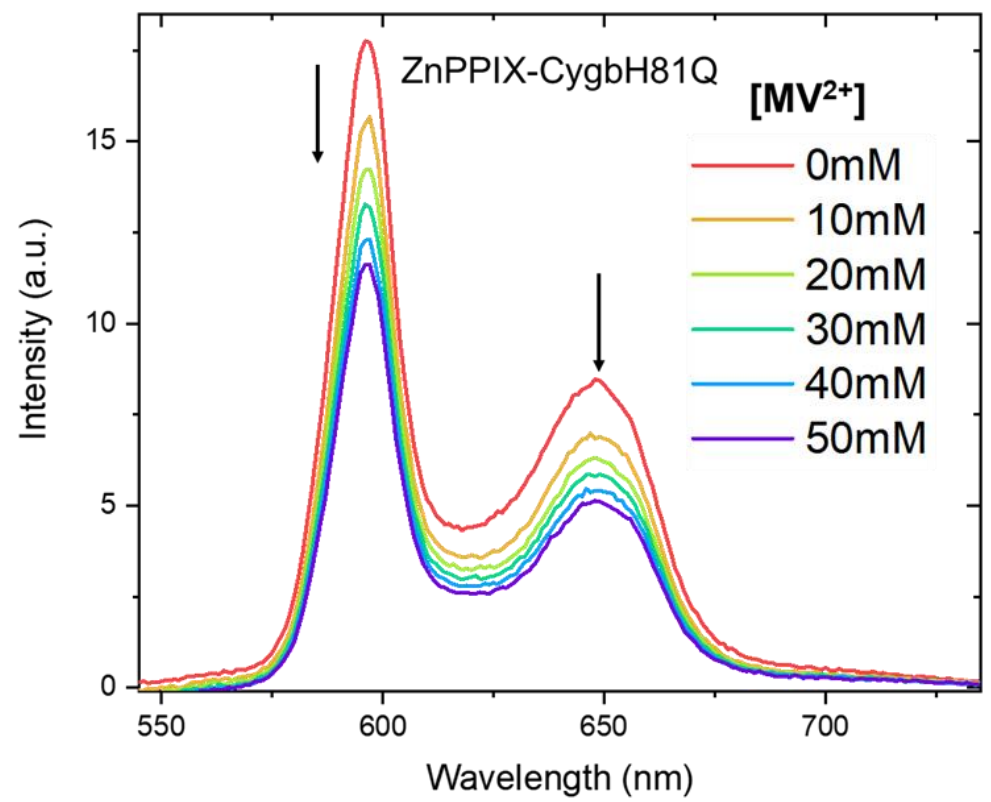

Figure 5.7. Example of fluorescence emission spectra of ZnPPIX reconstituted protein in the presence of increasing concentration of methyl viologen.

The quenching of the ZnPPIX reconstituted globins was probed by monitoring the fluorescent emission spectra at increasing concentration of the quencher (Figure 5.7). The Stern-Volmer plot was constructed by potting the ratio of the emission intensity at zero quencher and increased quencher concentration as a function of quencher concentration and the plots are shown in Figure 5.8. The Stern-Volmer constant, $\mathrm{K}_{\mathrm{sv}}$, was determined by fitting the experimental data using a linear Stern-Volmer equation and the results are summarized in Table 5.4. The value of $\mathrm{K}_{\mathrm{sv}}$ was determined to be 16.7 
$\pm 0.9,17.2 \pm 5.3$, and $9.62 \pm 1.1 \mathrm{M}^{-1}$ for ZnPPIX reconstituted CygbWT, CygbC38S, CygbH81Q, respectively. Also, ZnPPIX reconstituted NgbWT has relatively larger Ksv value of $34.1 \pm 2.1 \mathrm{M}^{-1}$ while the ZnPPIX-NgbC46G and ZnPPIX-NgbH64Q showed a smaller $\mathrm{K}_{\mathrm{sv}}$ values of $26.5 \pm 0.76$ and $25.4 \pm 11.0 \mathrm{M}^{-1}$, respectively.

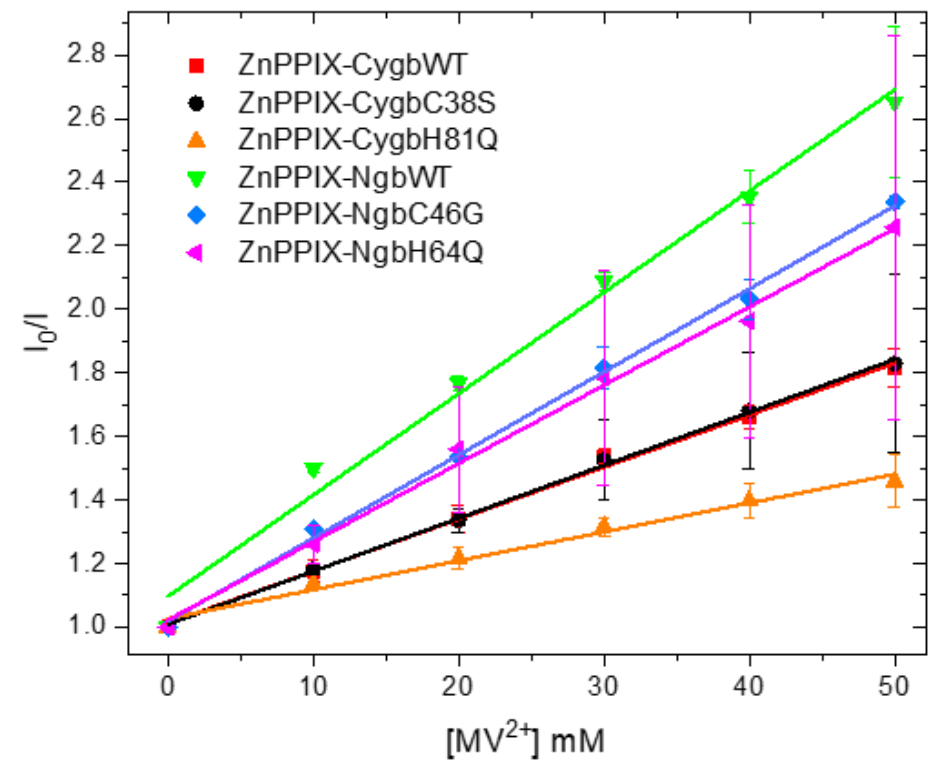

Figure 5.8. The Stern-Volmer plot for the quenching of the steady-state fluorescence of ZnPPIX reconstituted globins by methyl viologen.

In addition to monitoring quenching of the ZnPPIX singlet state, we have taken advantage of the fact that $\mathrm{O}_{2}$ is an excellent quencher of ZnPPIX triplet state. The

\begin{tabular}{|l|c|}
\hline \multicolumn{1}{|c|}{ Proteins } & $\mathrm{K}_{\text {sv }}\left(\mathrm{M}^{-1}\right)$ \\
\hline ZnPPIX-CygbWT & $16.7 \pm 0.9$ \\
\hline ZnPPIX-CygbC38S & $17.2 \pm 5.3$ \\
\hline ZnPPIX-CygbH81Q & $9.62 \pm 1.1$ \\
\hline ZnPPIX-NgbWT & $34.1 \pm 2.1$ \\
\hline ZnPPIX-NgC46G & $26.5 \pm 0.76$ \\
\hline ZnPPIX-NgbH64Q & $25.4 \pm 11.0$ \\
\hline
\end{tabular}

Table 5.4. Summary of Stern-Volmer constant of ZnPPIX-reconstituted hexacoordinate globins. quenching of the triplet state of ZnPPIX reconstituted globins was characterized by measuring the phosphorescence lifetime. The quantum yield of phosphorescence 
emission was very low, and the triplet state emission was usually detected at low temperatures (around $100 \mathrm{~K}$ ), making the detection of the triplet state lifetime challenging. Here, we took advantage of the fact that ZnPPIX triplet state absorbs at $447 \mathrm{~nm}$ and thus the triplet state lifetime can be detected by measuring the time profile of the triplet state absorbance.

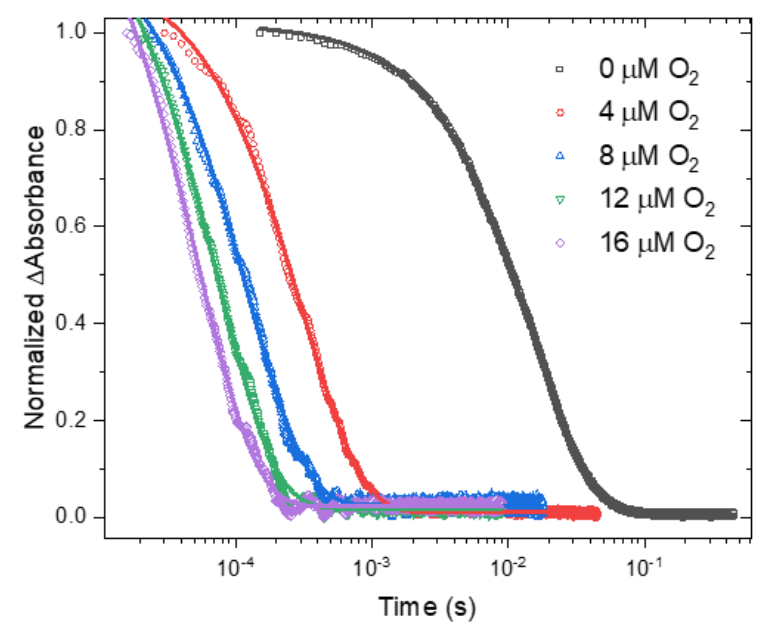

Figure 5.9. Triplet state lifetime traces of $\mathrm{ZnPPIX}-\mathrm{hhMb}$ obtained by transient absorbance at varying concentration of oxygen. All traces are fitted by single exponential decay.

The triplet state quenching was monitored by measuring the triplet state lifetime as a function of the increasing concentration of oxygen. The transient absorption traces

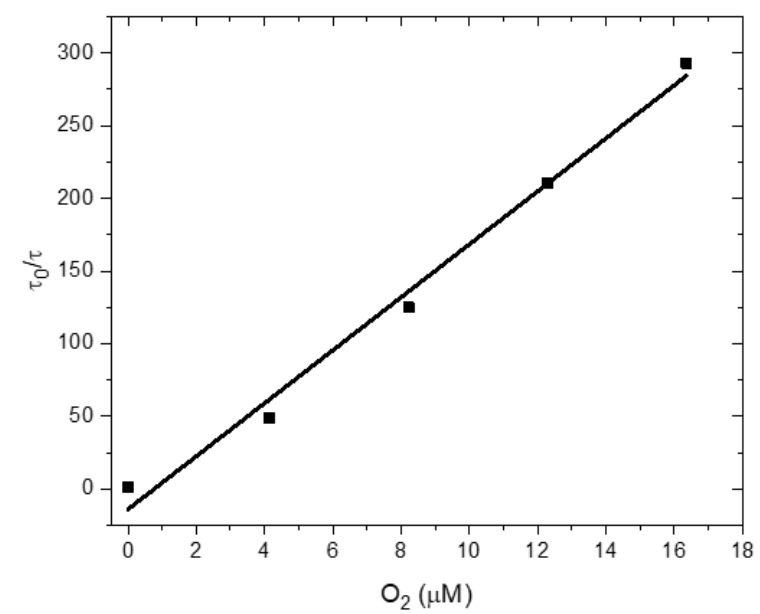

Figure 5.10. The Stern-Volmer plot for the quenching of the triplet-state phosphorescence of ZnPPIX reconstituted hhM by oxygen. 
for ZnPPIX-hhMb are shown in Figure 5.9 and the corresponding Stern Volmer plot is presented in Figure 5.10. The quenching rate constant were determined from the plot of the ratio of the triplet state lifetime in the absence of quencher and in the presence of quencher. The recovered rate constants are: $\mathrm{k}_{\mathrm{q}},=3.23 \times 10^{8} \pm 0.32 \mathrm{M}^{-1} \mathrm{~s}^{-1}$ for ZnPPIX$\mathrm{hhMb}$ which is similar to the previous

\begin{tabular}{|l|l|}
\hline & $\mathrm{k}_{\mathrm{q}}\left(\mathrm{M}^{-1} \mathrm{~s}^{-1}\right)$ \\
\hline ZnPPIX-hhMb & $3.23 \times 10^{8} \pm 0.32$ \\
\hline ZnPPIX-CygbWT & $3.03 \times 10^{8} \pm 0.8$ \\
\hline ZnPPIX-NgbWT & $4.08 \times 10^{8} \pm 1.39$ \\
\hline
\end{tabular}

Table 5.5. Summary of quenching rate constant of ZnPPIX-reconstituted globins.

reported value [Barboy and Feitelson]. Also, the $\mathrm{k}_{\mathrm{q}}$ for ZnPPIX-CygbWT is $3.03 \times 10^{8}$ $\pm 0.8 \mathrm{M}^{-1} \mathrm{~s}^{-1}$ and ZnPPIX-NgbWT is $4.08 \times 10^{8} \pm 1.39 \mathrm{M}^{-1} \mathrm{~s}^{-1}$ that is comparable to the value obtained for ZnPPIX reconstituted $h \mathrm{hMb}$.

\subsection{Discussion}

The reconstitution of globins with ZnPPIX combined with site-directed mutagenesis provides a powerful method to study how the prosthetic group interacts with the protein scaffold as well as with the exogenous ligands due to the high sensitivity of the fluorescent analog, ZnPPIX, to changes in its surrounding as well as to the presence of various quenchers Steady-state absorption and fluorescence emission of the ZnPPIX-reconstituted proteins provide insight into the structural heterogeneity of the heme binding cavity. An $\alpha$ - band split was observed for most Cygb reconstituted 
proteins while the split is compromised in CygbR84L mutant and nearly disappeared in CygbH81L mutant, suggesting that the electronic structure of ZnPPIX is sensitive to small changes in the heme binding pocket. Namely, the conformational heterogeneity of the distal histidine sidechain as well as two distinct orientations of Arg84 sidechain observed in the structure of ligand bond Cygb may contribute to the observed split of $\alpha$-band in the absorption spectrum of Cygb. Compare to emission spectra of ZnPPIXhhMb and ZnPPIX-Cygb variants, all ZnPPIX reconstituted Ngb variants shown a blueshifted Soret bands by $3 \mathrm{~nm}$. Such hypsochromic shift in the absorption spectra may be attributed to an increase in the polarity of the fluorophore surrounding, thus the heme cavity in Ngb appears to be more polar compare to that of Mb and Cygb [(Leonard, Yonetani, and Callis]. This is consistent with the distinct emission spectra measured for $\mathrm{Ngb}$ variants and Cygb variant as the longer wavelength emission peak in $\mathrm{Ngb}$ is blue shifter compare to $\mathrm{Cygb}$ and $\mathrm{Mb}$. Considering a larger volume of the heme binding cavity in Ngb compare to Cygb, the Ngb distal cavity may be occupied by water molecule(s), resulting in the blue shift of the Soret band and the longer wavelength peak of the emission spectrum. Interestingly, Ngb C46G mutant and Ngb E52Q mutant leads to a 1-2 $\mathrm{nm}$ blue shift in the longer wavelength emission peak compare to NgbWT, suggesting that the heme pocket in $\mathrm{Ngb}$ is sensitive to the structural changes in the $\mathrm{CD}$ loop that are triggered by to the reduction of the disulfide bridge or substitution of negatively charged Glu residue by Gln. 
Additional information about the heme pocket dynamics can be obtained by monitoring the fluorescence lifetime of ZnPPIX. Based on the biphasic fluorescent lifetimes and associated pre-exponential values, it is reasonable to assume there are two different orientations of the heme inside the distal cavity in both Cygb and $\mathrm{Ngb}$. Indeed, heme disorder was observed in previous NMR and X-ray crystallography studies on human and murine $\mathrm{Ngb}$ as the heme group was found to rotate around the $\alpha-\gamma$-meso axis [Xu, Yin, and Du; Du et al.; Pesce et al.]. The data reported here suggest that the orientation of the porphyrin group in the reconstituted $\mathrm{Ngb}$ variants is sensitive to the conformational changes in the $\mathrm{CD}$ loop of the protein, as the lifetime data for NgbC46Gmutnt and NgbH64Q mutant exhibit increase in $\tau_{1}$ and $\tau_{2}$ values with respect to the values observed for NgbWT. Interestingly, the pre-exponential factor associated with $\tau_{2}$ in NgbH64Q mutant is less than $10 \%$, suggesting that the distal histidine sidechain contributes to the observed bimodal lifetime in ZnPPIXNgb. This is further confirmed by lifetime data for NgbC46G mutant. Although two lifetimes were resolved in this construct, the pre-exponential factor associated with $\tau_{2}$ is small, $\sim 5 \%$, suggesting that removal of the intra-protein disulfide bridge promotes a single orientation of the heme group, possible through decreasing the conformational heterogeneity of the distal histidine. The lifetime data recorded for Cygb constructs indicate that the lifetime of ZnPPIX reconstituted in Cygb variants is less sensitive to the changes in the flurophore surrounding. Surprisingly, the lifetime parameters measured for CygbH81Q mutant are almost identical to lifetime data for CygbWT. The removal of the disulfide bridge leads 
to a small increase in both $\tau_{1}$ and $\tau_{2}$ as well as the increase in the pre-exponential factor for $\tau_{1}$ and similar increase in $\tau_{1}$ was observed for CygbR84L mutant and truncated mutant. Interestingly, previous study reported that arginine 84 adopt two distinct orientations in the ligand free Cygb: a major conformation $(\sim 60 \%)$ with the side-chain positioned towards the solvent and a minor conformation $(\sim 40 \%)$ with the side-chain positioned towards the heme pocket, and this heterogeneity might be promoted by the reduction of the disulfide bridge (Astudillo et al., 2013). Also, the lifetime data for the ZnPPIX reconstituted truncated Cygb indicates that the extended terminals in Cygb can also impact the heme orientation in the heme binding cavity, however, the mechanism is not clear. The phosphorescence lifetimes are comparable in all ZnPPIX reconstituted globins suggesting that the triplet state lifetime is not sensitive to small changes in the fluorophore environment. It is also possible that the changes in the phosphorescent decay are small and thus may be difficult to resolved as the phosphorescence lifetime is strongly impacted by a possible presence of residual oxygen.

Quenching study can provide information on the accessibility and migration of the ligand to the heme pocket. The quenching of the steady-state fluorescence of ZnPPIX reconstituted globins by methyl viologen illustrate distinct distal pocket accessibility in Cygb and $\mathrm{Ngb}$. The $\mathrm{K}_{\mathrm{sv}}$ value reveals that Cygb variants are generally less accessible for quencher than Ngb variants. The replacement of distal histidine in Cygb further decreased the accessibility of the distal pocket while the removal of the internal disulfide bond does not impact the quencher access to ZnPPIX in Cygb. Compare to 
Cygb, the distal cavity in Ngb is more open to the exogenous ligands, but the accessibility is diminished by the removal of the disulfide bridge and by substitution of distal histidine residue. Oxygen quenching of the triplet-state phosphorescence of ZnPPIX reconstituted globin provide the quenching rate constant for ZnPPIX reconstituted hhMb, CygbWT and NgbWT. Although there are small variations, the quenching rate constant determined for all three proteins are comparable and indicate that the diffusion of the oxygen molecule represent the rate limiting step for $\mathrm{O}_{2}$ migration into the heme binding pocket.

\subsection{Summary}

In conclusion, by incorporating heme fluorescent analogs into the hexacoordinated globins, we were able to reveal distinct structural changes in hexa-coordinate globins Cygb and Ngb. The observed bimodal fluorescence lifetime as well as the splitting of the $\alpha$-band in the absorption spectrum of Cygb variants indicate heterogeneity of the ZnPPIX orientation in the protein pocket and/or heterogeneity in the fluorophore surrounding. Indeed, the results obtained for constructs with replaced distal histidine residue in both proteins suggest that the orientation of the distal histidine may contribute to the conformational heterogeneity observed in hexa-coordinate globins. In addition, the distal histidine conformation may be modulated by the disulfide bond in Ngb and by Arg81 sidechain in Cygb protein. The environment of the ZnPPIX in Ngb and Cygb is distinct as evident from different absorption and emission spectra recorded for 
ZnPPIX Ngb and ZnPPIX Cygb variants. This is further confirmed by an increased accessibility of ZnPPIX to $\mathrm{MV}^{2+}$ quencher in $\mathrm{Ngb}$ structure. 


\section{THE $\mathrm{O}_{2}$ BINGING KINETIC AND IMPACT ON HEME CAVITY STABILITY IN GLOBIN X.}

\subsection{Introduction}

Globin $\mathrm{X}(\mathrm{GbX})$ is a new member of the globin superfamily that first discovered in fish and amphibians (Roesner et al., 2004). Similar to other novel members of the vertebrate globins such as neuroglobin ( $\mathrm{Ngb}$ ) and cytoglobin (Cygb), GbX exhibits a hexa-coordination that has a distal histidine bind the heme iron at six position (Blank, Wollberg, et al., 2011). The distal histidine in GbX can be displaced by the small diatomic ligands such as $\mathrm{CO}, \mathrm{NO}$, or $\mathrm{O}_{2}$. As a hexa-coordinate globin, the distal histidine in the heme pocket will compete with the exogenous ligands which ultimate modulate the ligand affinity to the protein, for example, replace of the distal histidine in $\mathrm{Ngb}$ result in a 5 to 10 fold increase in $\mathrm{CO}$ rebinding rate compare to $\mathrm{WT}$ and the replacement of the distal histidine in mouse $\mathrm{Ngb}$ increases the affinity of the protein for $\mathrm{O}_{2} 10$ fold. GbX display a high affinity to $\mathrm{O}_{2}\left(\mathrm{P}_{50} \sim 1.3\right.$ torr $)$ which is close to that observed for $\mathrm{Mb}$ ( $\mathrm{P}_{50} \sim 1$ torr), $\mathrm{Cygb}\left(\mathrm{P}_{50} \sim 1\right.$ torr $)$ and $\mathrm{Ngb}\left(\mathrm{P}_{50} \sim 1.9\right.$ torr), suggesting its potential role as a respiratory protein (Blank, Wollberg, et al., 2011).

In addition, $\mathrm{GbX}$ possess cysteine residues that are capable of forming intramolecular disulfide bridge which may influence the overall oxygen affinity. Indeed, Burmester's group has shown that in the presence of the reducing agent, DTT, GbX exhibits a fourfold decrease in the affinity for $\mathrm{O}_{2}$ (Blank, Wollberg, et al., 2011). 
Similar impact of the disruption of disulfide bridge on $\mathrm{O}_{2}$ affinity was also observed in $\mathrm{Ngb}$ ( nine-fold) but small impact of the disulfide bond on $\mathrm{O}_{2}$ was observed in Cygb, two fold (Hamdane et al., 2003). Such large change in the $\mathrm{O}_{2}$ affinity observed in Ngb was attributed to the movement of the E-helix triggered by the disruption of the disulfide bridge. The presence of intramolecular disulfide bridge is also important for other function properties of globins. For example, Cygb cannot bind lipids int the absence of the disulfide bond (Beckerson, Wilson, et al., 2015). However, the disruption of the disulfide bridge in $\mathrm{Ngb}$ does not impact the rate constant for $\mathrm{CO}$ escape from the protein matrix (Astudillo et al., 2010). Less is known about the impact of the intramolecular disulfide bridge on the structural properties of $\mathrm{GbX}$ as the crystal structure of GbX has not been resolved. The two cysteines in the GbX that are responsible for the disulfide bridge formation are the Cys65 and Cys141, The Cys residues are located apart compare to Ngb structure that has Cys residue that form a disulfide bridge located in the CD loop, Cys 46 and Cys 55. Therefore, the disruption of the internal disulfide bridge in the GbX may trigger larger structural changes and thus have more pronounce impact on functional properties of GbX. Here, we employed the time-resolved absorption spectroscopy and photoacoustic calorimetry together with site-directed mutagenesis to investigate the kinetic and energetics for $\mathrm{O}_{2}$ binding to $\mathrm{GbX}$ and how the internal disulfide bridge can regulate the $\mathrm{O}_{2}$ binding kinetics and thermodynamics. Since the biological and physiological function of the GbX is still not clear, out study can provide information on how $\mathrm{GbX}$ interacts with the biological 
important molecule, $\mathrm{O}_{2}$, in terms of the affinity, rate constants and activation and reaction thermodynamic parameters.

\subsection{Result}

\subsubsection{Steady-state UV-vis spectra}

The $\mathrm{O}_{2}$ bound GbXWT and C65A mutant absorption spectra were recorded and are shown in figure 6.1 and summarized in table 6.1 .
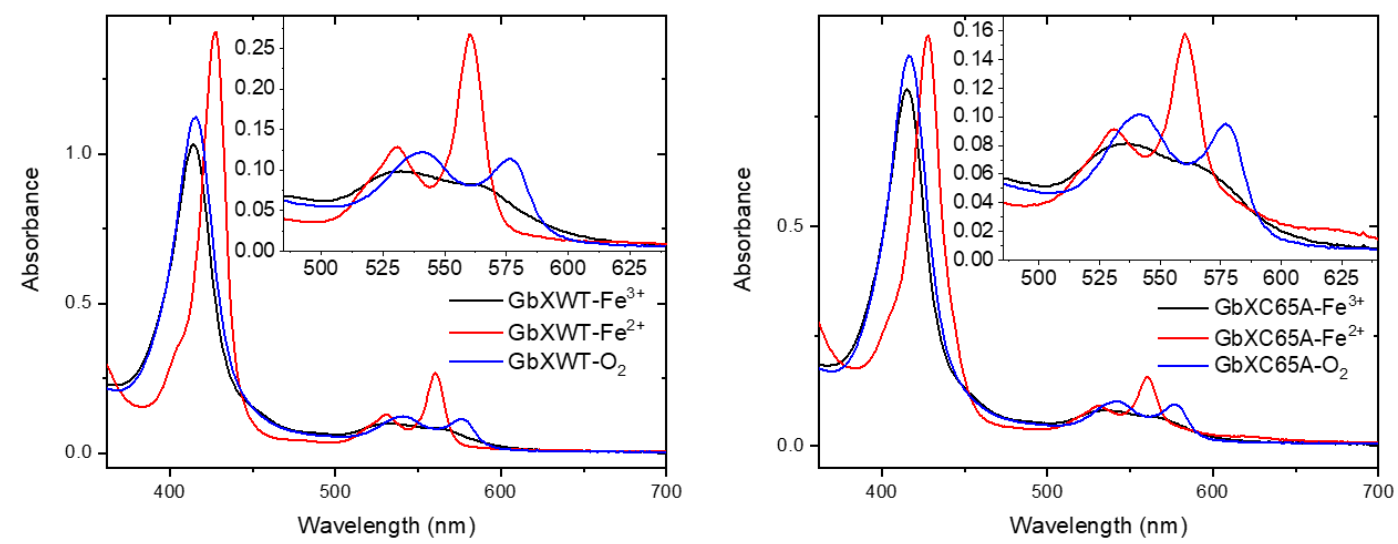

Figure 6.1. Absorption spectra of the oxidized (met), reduced (ferrous deoxy), and $\mathrm{O}_{2}$ bound forms of GbX WT and C65A mutant. Measured in $50 \mathrm{mM}$ TrisHCl pH 7.0.

The $\mathrm{O}_{2}$ bound $\mathrm{GbXWT}$ shows a Soret at $416 \mathrm{~nm}$ and two additional Q-bands at $541 \mathrm{~nm}$ and $574 \mathrm{~nm}$. The Soret band is shifted $6 \mathrm{~nm}$ and the $\beta$-bands shifted $\sim 1 \mathrm{~nm}$ and

\begin{tabular}{|l|c|c|c|}
\hline & Soret band (nm) & $\beta$-band (nm) & $\alpha$-band (nm) \\
\hline GbXWT-Fe $^{3+}$ & 414 & 532 & 563 \\
\hline GbXWT-Fe $^{2+}$ & 428 & 531 & 560 \\
\hline GbXWT-O $_{2}$ & 416 & 541 & 576 \\
\hline GbXC65A-Fe $^{3+}$ & 415 & 536 & 564 \\
\hline GbXC65A-Fe $^{2+}$ & 428 & 531 & 560 \\
\hline GbXC65A-O $_{2}$ & 416 & 541 & 577 \\
\hline
\end{tabular}

Table 6.1. Absorption spectra wavelength of Soret and Q-band of GbX WT and C64A mutant summarized in the table.

$\alpha$-band shifted $5 \mathrm{~nm}$ compare to $\mathrm{CO}$ bound GbXWT. Absorption spectrum of $\mathrm{O}_{2}$ bound

GbXC65A has the Soret band at $416 \mathrm{~nm}$ and Q-bands at $541 \mathrm{~nm}$ and $577 \mathrm{~nm}$ and it is 
comparable to the absorption spectrum for GbXWT, suggesting that the heme electronic structure is similar in the oxygen bound protein with and without intra-protein disulfide bridge.

\subsubsection{Stability of $\mathrm{O}_{2}$ bound GbX towards $\mathrm{pH}$ unfolding}

The stability of $\mathrm{O}_{2}$ bound $\mathrm{GbX}$ variants was probed by recoding absorbance of the Soret band as a function of $\mathrm{pH}$. The overlay of the absorption spectra of $\mathrm{O}_{2}$ bound GbXWT measured in the $\mathrm{pH}$ range from 8.0 to 0.4 is shown in figure 6.2 , The absorption spectra show an isosbestic point at $393 \mathrm{~nm}$, pointing towards a two-state heme release from the oxygen bond protein.

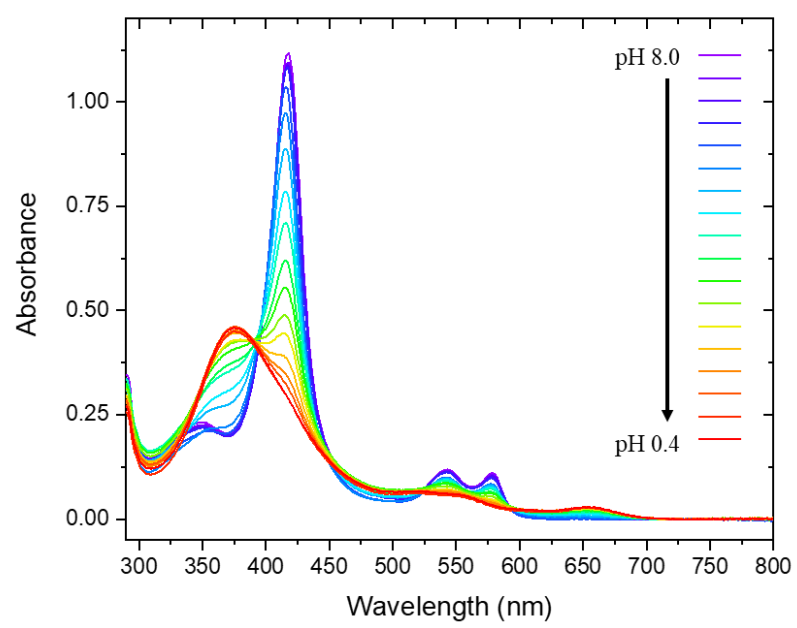

Figure 6.2. UV-vis absorption spectra of GBXWT in the oxygen bound form as function of $\mathrm{pH}$. Measurements were performed in $5 \mathrm{mM}$ phosphate-citrate buffer and $100 \mathrm{mM} \mathrm{NaCl}$, under equilibrium conditions.

The absorption spectrum of GbXWT measured at $\mathrm{pH} 0.4$ shows a broad Soret band with a maximum at $375 \mathrm{~nm}$ which is consistent with an destabilize polypeptide chain and a concomitant release of the prosthetic group from the protein matrix. Analogous changes in the absorption spectra were observed for $\mathrm{pH}$ unfolding of GbXC65A (data 
not shown). The fraction of unfolded protein calculated based on the absorbance changes at $416 \mathrm{~nm}$ was plotted as a function of $\mathrm{pH}$ for both WT and C65A mutant (Figure 6.3). The experimental data were fitted using Eq. 3.41 and the fitting parameters are summarized in Table 6.2. The $\mathrm{pH}$ induced unfolding of $\mathrm{O}_{2}$ bound GbXWT is

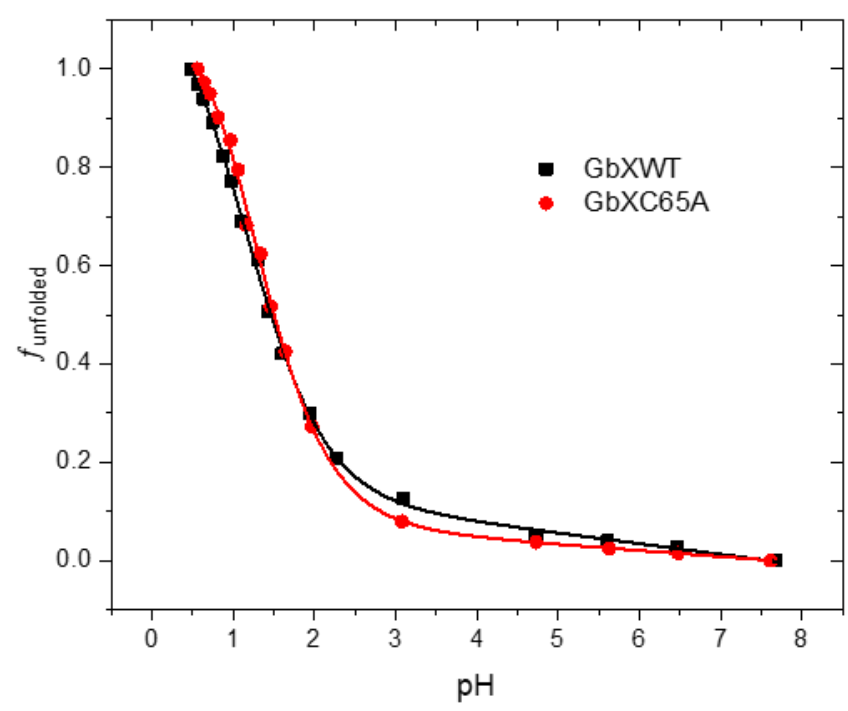

Figure 6.3. Fraction unfolding of oxygen bound GbX WT and C65A mutant plot as a function of $\mathrm{pH}$.

characterized by a $\mathrm{pH}$ at half-transition $\left(\mathrm{pH}_{\text {mid }}\right)$ of 1.19 , which is about 3.4 units lower than that previously reported for met horse heart $\mathrm{Mb}\left(\mathrm{pH}_{\text {mid }} 4.6\right)$ and 2 units lower than that determined for met $\mathrm{Ngb}\left(\mathrm{pH}_{\text {mid }}\right.$ 3.2). Also, the $\mathrm{pH}$ induced unfolding of $\mathrm{O}_{2}$ bound GbXWT is 0.7 unit lower than that obtained for the met GbXWT ( $\left.\mathrm{pH}_{\text {mid }} 1.9\right)$. In

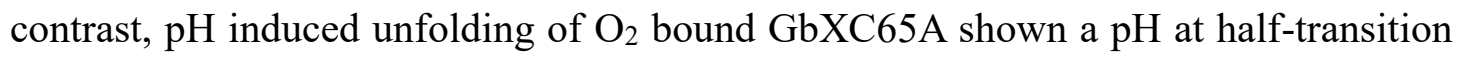
$\left(\mathrm{pH}_{\text {mid }}\right)$ of 1.07 which is similar to the value determined for $\mathrm{O}_{2}$ bound $\mathrm{GbXWT}$. 


\begin{tabular}{|c|c|c|}
\hline & $\mathrm{pH}_{\text {mid }}$ & $\Delta v_{\mathrm{H}^{+}}$ \\
\hline GbXWT & 1.19 & -0.90 \\
\hline GbXC65A & 1.07 & -0.97 \\
\hline
\end{tabular}

Table 6.2. Parameters of the acid-induced oxygen bound GbX variants unfolding.

Interestingly, the $\mathrm{pH}_{\text {mid }}$ for $\mathrm{pH}$ induced unfolding of $\mathrm{O}_{2}$ bound $\mathrm{GbXC65 \textrm {A }}$ is 1.4 units lower than that for the met form of $\mathrm{GbXC} 65 \mathrm{~A}\left(\mathrm{pH}_{\text {mid }} 2.48\right)$, suggesting a minor impact of the disulfide bridge on the stability of the protein in the oxygen bound form.

\subsubsection{GbX $\mathrm{O}_{2}$ binding kinetic}

To further analyze interactions of $\mathrm{GbX}$ variants with $\mathrm{O}_{2}, \mathrm{O}_{2}$ binding kinetics were investigated using transient absorption spectroscopy. The transient absorption traces for $\mathrm{O}_{2}$ binding to $\mathrm{GbX}$ constructs studied are shown in Figure 6.4A. Both traces were fitted
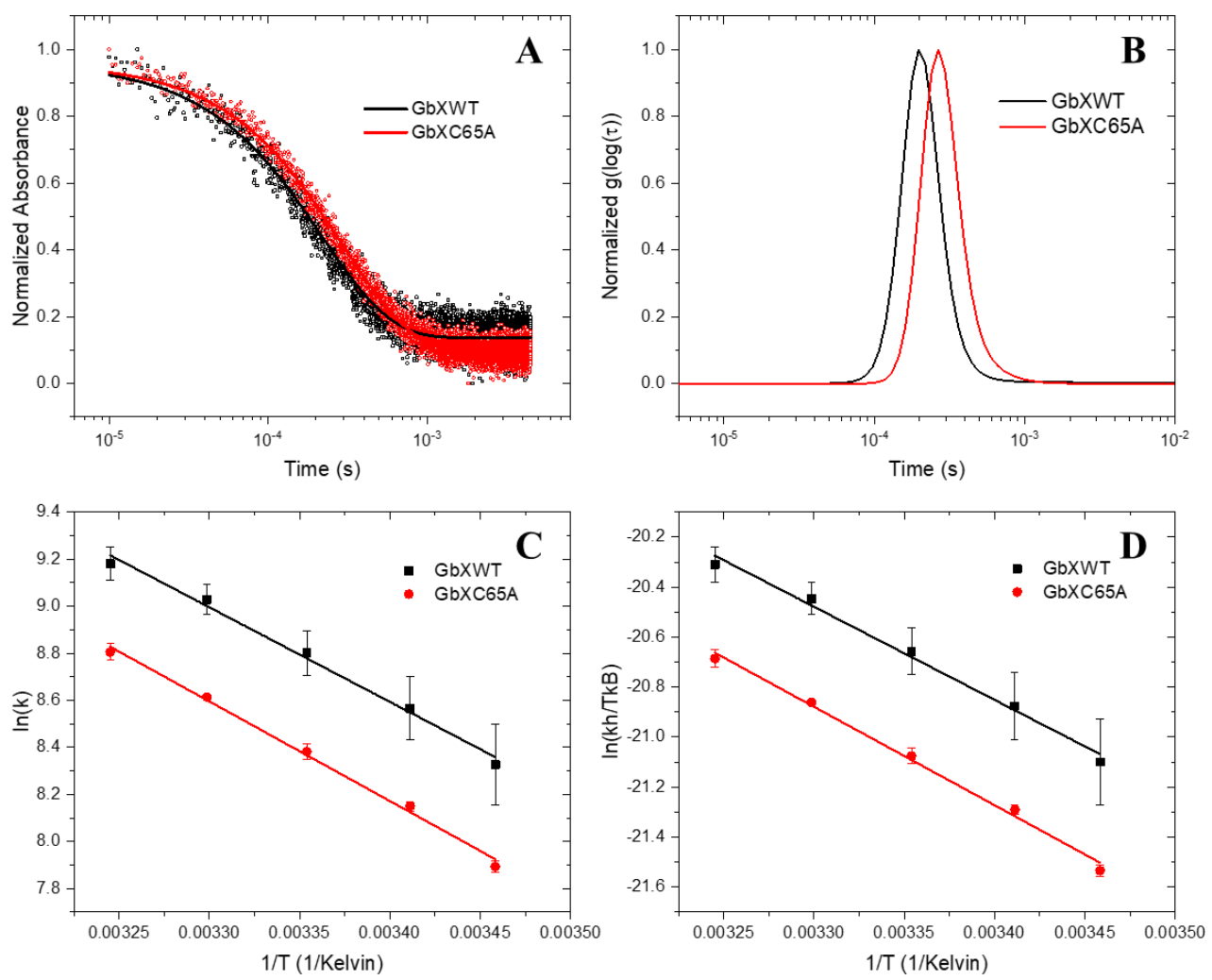

Figure 6.4. A, transient absorption traces for $\mathrm{O}_{2}$ rebinding to $\mathrm{GbX}$ variants. $\mathrm{B}$, Lifetime 
distribution associated with the $\mathrm{O}_{2}$ rebinding to $\mathrm{GbX}$ variant determined by MEM approach. $\mathrm{C}$, Arrhenius plot of temperature dependent $\mathrm{O}_{2}$ rebinding to $\mathrm{GbX}$ variants. D, Eyring plot of temperature dependent $\mathrm{O}_{2}$ rebinding to $\mathrm{GbX}$ variants.

using a single exponential decay model and MEM (maximum entropy method) and the kinetic parameters are summarized in Table 6.3. A single kinetic was obtained for $\mathrm{O}_{2}$ association to GbXWT with $\mathrm{k}=21.2 \pm 2.8 \mu \mathrm{M}^{-1} \mathrm{~s}^{-1}$. Similar rate constant was obtained using MEM analysis $\left(\mathrm{k}=22.0 \pm 2.4 \mu \mathrm{M}^{-1} \mathrm{~s}^{-1}\right)$. The analysis of $\mathrm{O}_{2}$ rebinding kinetic to GbXC65A mutant also reveals only 1 rate constant which is $13.9 \pm 0.3 \mu \mathrm{M}^{-1} \mathrm{~s}^{-1}$ which is slower than the rate constant measured for $\mathrm{O} 2$ binding to GbXWT. In addition to the individual rate constants, the activation energy, log pre-exponential factor, reaction enthalpy, and reaction entropy were determined by temperature dependent $\mathrm{O}_{2}$ rebinding experiment (figure 6.4 C, D; Table 6.4).

\begin{tabular}{|l|c|}
\hline & $\mathbf{k}\left(\mu \mathrm{M}^{-1} \mathbf{s}^{-1}\right)$ \\
\hline GbXWT (decy) & $21.2 \pm 2.8$ \\
\hline GbXWT (MEM) & $22.0 \pm 2.4$ \\
\hline GbXC65A (decy) & $13.9 \pm 0.3$ \\
\hline GbXC65A (MEM) & $14.6 \pm 0.3$ \\
\hline
\end{tabular}

Table 6.3. Rate constants for $\mathrm{O}_{2}$ binding to $\mathrm{GbX}$ variants obtained using both exponential decay model and MEM analysis at $20^{\circ} \mathrm{C}$.

The activation energy for $\mathrm{O}_{2}$ binding to $\mathrm{GbX}$ is $7.9 \pm 1.5 \mathrm{kcal} \mathrm{mol}^{-1}$ which is similar to horse heart $\mathrm{Mb}\left(7.5 \mathrm{kcal} \mathrm{mol}^{-1}\right)$ and human $\mathrm{Ngb}\left(8 \mathrm{kcal} \mathrm{mol}^{-1}\right)$ as well as the hemoglobin from Drosophila (10.5 $\left.\mathrm{kcal} \mathrm{mol}^{-1}\right)$ and Arabidopsis $\left(8 \mathrm{kcal} \mathrm{mol}^{-1}\right)$, indicating a similar energy barrier for $\mathrm{O}_{2}$ binding to heme iron in globins (Julien Uzan et al, 2004). 


\begin{tabular}{|c|c|c|c|c|}
\hline & $\mathrm{E}_{\mathrm{a}}\left(\mathrm{kcal} \mathrm{mol}^{-1}\right)$ & $\log (\mathrm{A})$ & $\Delta \mathrm{H}^{\ddagger}\left(\mathrm{kcal} \mathrm{mol}^{-1}\right)$ & $\Delta \mathrm{S}^{\ddagger}\left(\mathrm{cal} \mathrm{mol}^{-1} \mathrm{~K}^{-1}\right)$ \\
\hline GbXWT & $7.9 \pm 1.5$ & $9.6 \pm 1.1$ & $7.3 \pm 1.5$ & $-16.1 \pm 4.6$ \\
\hline GbXC65A & $8.4 \pm 0.1$ & $9.8 \pm 0.1$ & $7.8 \pm 0.1$ & $-15.3 \pm 0.3$ \\
\hline
\end{tabular}

Table 6.4. Activation energy, log pre-exponential factor, activation enthalpy, and activation entropy of temperature dependent $\mathrm{O}_{2}$ rebinding to $\mathrm{GbX}$ variants.

Also, activation enthalpy and entropy were obtained from the Eyring plot for $\mathrm{O}_{2}$ binding to $\mathrm{GbX}$. The linar fit provide $\Delta \mathrm{H}^{*}$ to be $7.3 \pm 1.5 \mathrm{kcal} \mathrm{mol}^{-1}$ and $\Delta \mathrm{S}^{*}$ equal to $-16.1 \pm$ 4.6 cal $\mathrm{mol}^{-1} \mathrm{~K}^{-1}$. In comparison, disulfide bridge mutant GbXC65A shown an activation energy $\left(8.4 \pm 0.1 \mathrm{kcal} \mathrm{mol}^{-1}\right)$, activation enthalpy $\left(\Delta \mathrm{H}^{\ddagger}=7.8 \pm 0.1 \mathrm{kcal} \mathrm{mol}^{-}\right.$

$\left.{ }^{1}\right)$ as well as activation entropy $\left(-15.3 \pm 0.3 \mathrm{cal} \mathrm{mol}^{-1} \mathrm{~K}^{-1}\right)$ for $\mathrm{O}_{2}$ binding which are comparable to GbXWT.

\subsubsection{Photoacoustic calorimetry result of $\mathrm{O}_{2}$ dissociation}

The bimolecular quantum yiled for $\mathrm{O}_{2}$ photo-release from GbXWT and C65A mutant is obtained by transient absorption spectroscopy and plot as a function of temperature shown in figure 6.5. The quantum yiled of $\mathrm{GbXWTO}_{2}$ is ranging from $3.1 \%$ to $4.6 \%$ from $16^{\circ} \mathrm{C}$ to $35^{\circ} \mathrm{C}$. On other hand, the bimolecular quantum yield for $\mathrm{O}_{2}$ dissociation for GbXC65A is also temperature dependent and roughly two times higher than that observed for GbXWT. 


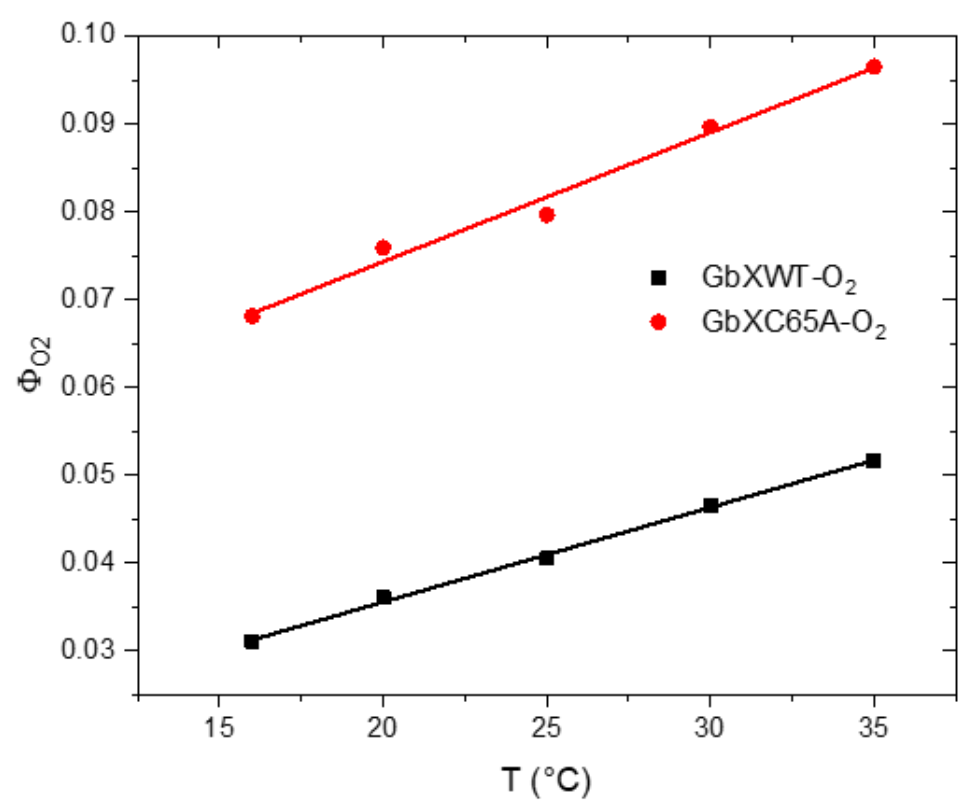

Figure 6.5 Quantum yiled of $\mathrm{O}_{2}$ from GbXWT and C65A plot as a function of temperature.

An overlay of the normalized photoacoustic traces for photo-dissociation of $\mathrm{O}_{2}$ from

GbXWT and GbXC65A together with the reference compound, 4SP, are shown in

Figures 6.6. The photoacoustic traces are clearly shown that there is no phase shift between the sample and reference which indicated that the $\mathrm{O}_{2}$ escaped from the protein matrix within $50 \mathrm{~ns}$.
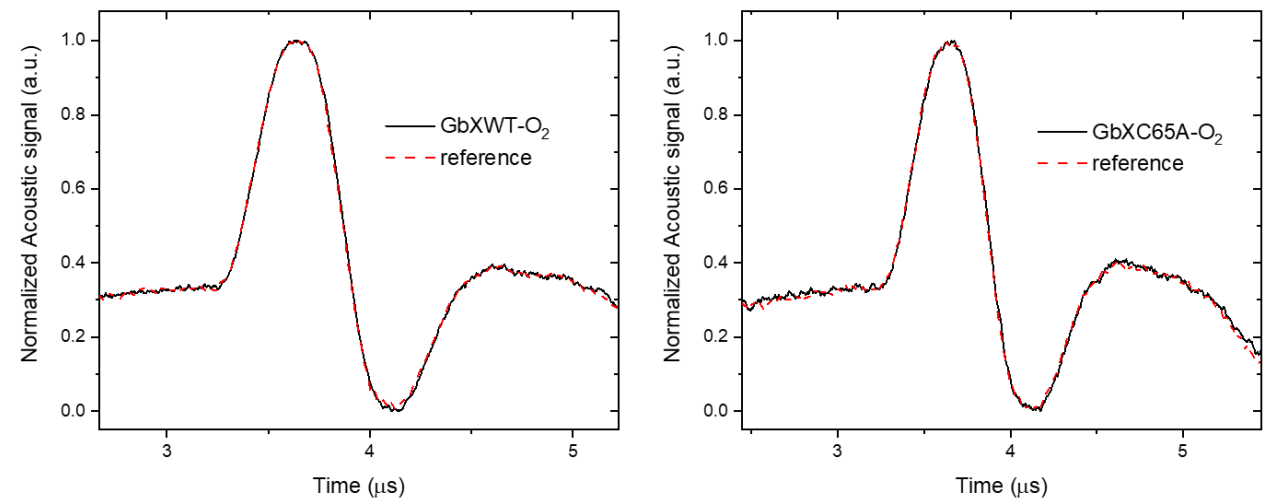

Figure 6.6 Photoacoustic traces for oxygen dissociation from GbXWT (left) and GbXC65A (right) together with the trace for the reference compound 4SP. Conditions: $20 \mu \mathrm{M}$ protein in $50 \mathrm{mM}$ Tris buffer ( $\mathrm{pH}$ 7.0). The absorbance of the reference compound is aligned with the absorbance of the sample at $532 \mathrm{~nm}$. 
Plots of the PAC amplitude ratios as a function of the thermal coefficient $[(\mathrm{Cp} \rho) / \beta]$ over the temperature range of $16-30{ }^{\circ} \mathrm{C}$ are displayed in Figure 6.7. The reaction enthalpy and volume changes were obtained from the intercept and slop of the linear plot, respectively, as described in the Materials and Methods section, and are listed in Table 6.5. From the reaction volume change, the structural volume change, $\Delta \mathrm{V}_{\text {str }}$, that describes the difference between the partial molar volume of the penta-coordinate GbX and $\mathrm{O}_{2}$-bound $\mathrm{GbX}$ can be determined according to the equation 3.49.

$$
\Delta V_{\text {total }}=\Delta V_{O_{2}}^{\emptyset}+V_{5 c}^{\emptyset} G b X-V_{6 c}^{\emptyset} G b X O_{2}
$$

Where $V_{O_{2}}^{\emptyset}$ is the partial molar volume of $\mathrm{O}_{2}\left(33.2 \mathrm{~mL} \mathrm{~mol}^{-1}\right), \mathrm{V}_{s t r}=V_{5 c}^{\emptyset} G b X-$ $V_{6 c}^{\emptyset} G b X O_{2}$ (Moore et al., 1982).

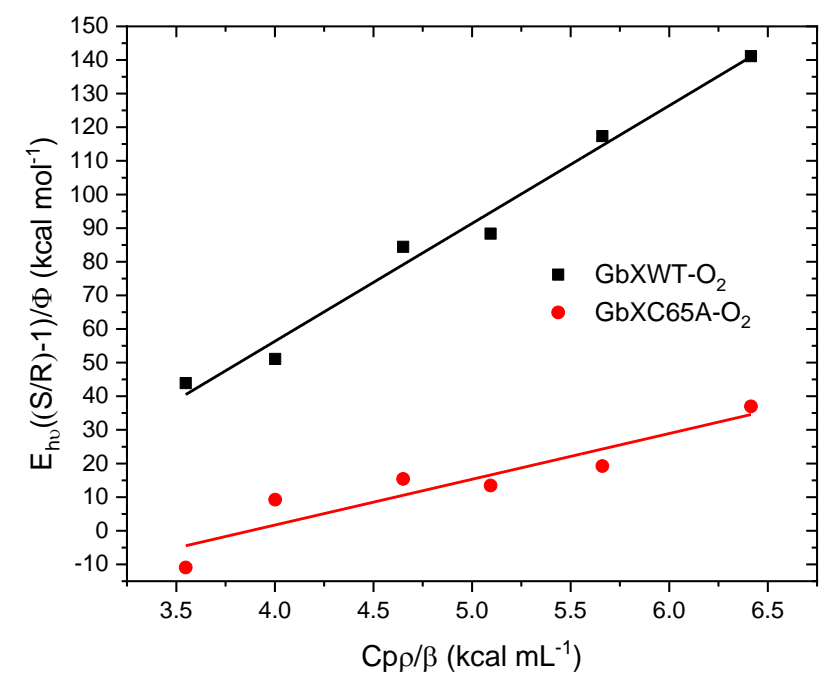

Figure 6.7. Plot of $\varphi$ Ehv as a function of $\mathrm{Cp} \rho / \beta$ for $\mathrm{O}_{2}$ photo-release from GbXWT (black), and GbXC65A (red). The associated volume and enthalpy changes were obtained from the slope and intercept of the linear fits, respectively.

The photo-dissociation of $\mathrm{O}_{2}$ from GbXWT is associated with a $\Delta \mathrm{V}_{\text {str }}$ of $0.8 \pm 12$ $\mathrm{mL} \mathrm{mol}{ }^{-1}$. However, the replacement of Cys65 with Ala has a significant impact on the structure volume change $\left(\Delta \mathrm{V}_{\mathrm{str}}=-18.2 \pm 2 \mathrm{~mL} \mathrm{~mol}^{-1}\right)$ likely because of a larger 
structural reorganization of the distal pocket in this mutant. The total enthalpy change for the photo-dissociation of $\mathrm{O}_{2}$ from GbXWT is $79 \pm 30 \mathrm{kcal} \mathrm{mol}^{-1}$, and $\mathrm{GbXC65 \textrm {A }}$ is $60 \pm 3 \mathrm{kcal} \mathrm{mol}^{-1}$.

\begin{tabular}{|c|c|c|c|c|c|}
\hline & Temperature $\left({ }^{\circ} \mathrm{C}\right)$ & $\underset{\left(\mathrm{kcal} \mathrm{mol}^{-1}\right)}{\Delta \mathrm{H}_{\text {total }}}$ & $\underset{\left({\left.\mathrm{kcal} \mathrm{mol}^{-1}\right)}^{\Delta \mathbf{H}_{\text {tr }}}\right.}{ }$ & $\begin{array}{c}\Delta \mathrm{V}_{\text {total }} \\
\left(\mathrm{mL} \mathrm{mol}^{-1}\right)\end{array}$ & $\begin{array}{c}\Delta \mathbf{V}_{\text {str }} \\
\left(\mathrm{mL} \mathrm{mol}^{-1}\right)\end{array}$ \\
\hline GbXWT-O & $16-30$ & $79 \pm 30$ & $65 \pm 30$ & $34 \pm 12$ & $0.8 \pm 12$ \\
\hline GbXC65A-O & $16-30$ & $60 \pm 3$ & $46 \pm 3$ & $15 \pm 2$ & $-18.2 \pm 2$ \\
\hline${ }^{*} \mathbf{M b}-\mathrm{O}_{2}$ & $6-15$ & $11.6 \pm 8$ & $-2.4 \pm 8$ & $2.5 \pm 0.5$ & $-30.7 \pm 0.5$ \\
\hline${ }^{*} \mathrm{Ngb}-\mathrm{O}_{2}$ & $16-35$ & $-23 \pm 4$ & $-37 \pm 4$ & $-9.0 \pm 0.7$ & -42.2 \\
\hline
\end{tabular}

Table 6.5. Thermodynamic parameters associated with $\mathrm{O}_{2}$ photo-dissociation from GbXWT and GbXC65A. (*(Astudillo, 2014))

\subsection{Discussion}

Out data exhibit several interesting findings on the interaction between $\mathrm{O}_{2}$ and

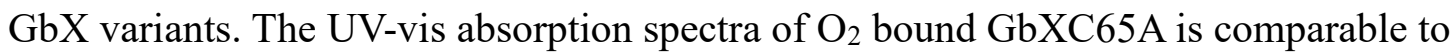
the $\mathrm{O}_{2}$ bound GbXWT indicate the electronic structure of the $\mathrm{O}_{2}$ bound heme is not affected by the presence or disruption of the disulfide bridge. GbX shows an unusual stability towards unfolding as the release of the heme group form the protein matrix occurs at very acidic $\mathrm{pH}$. The enhance stability of the heme group can be attributed to the presence of the oxygen bond between the distal histidine and the oxygen bound to the heme iron. Interestingly, the stability of the protein in the oxygen bond form is not impacted by the disulfide bride. Unlike $\mathrm{CO}$, the $\mathrm{O}_{2}$ binding to the heme iron can be modeled using a single exponential decay. This points towards distinct ligand binding mechanisms for $\mathrm{O}_{2}$ and $\mathrm{CO}$ in this protein. We speculate that several migration pathways are sampled during the $\mathrm{CO}$ binding whereas $\mathrm{O}_{2}$ may sample only a single 
migration pathway. However, we cannot exclude that the observed single binding kinetic for $\mathrm{O}_{2}$ association to the heme iron is due to the low amplitude of the absorption signal due to the small quantum yield for the bimolecular $\mathrm{O} 2$ rebinding. In addition, the activation energy of $\mathrm{O}_{2}$ binds to $\mathrm{GbX}$ variants is comparable to the activation energy for $\mathrm{O}_{2}$ binding to other globins, demonstrating a similar mechanism for $\mathrm{O}_{2}$ binding to heme in the globins. However, the activation entropy for $\mathrm{O}_{2}$ binding to $\mathrm{GbX}$ is $-16 \mathrm{cal}$ $\mathrm{mol}^{-1} \mathrm{~K}^{-1}$. This value is distinct from the activation entropy values determined for $\mathrm{O}_{2}$ binding to other globins such as human $\mathrm{Ngb}\left(2.8 \mathrm{cal} \mathrm{mol}^{-1} \mathrm{~K}^{-1}\right)$ or horse hear $\mathrm{Mb}(-0.9$ cal $\mathrm{mol}^{-1} \mathrm{~K}^{-1}$ ) (Uzan et al., 2004). Interestingly, the $\mathrm{O}_{2}$ rebinding rate determined by transient absorption spectroscopy demonstrate a slower rate constant for $\mathrm{O} 2$ binding to $\operatorname{GbXC65A}\left(13.9 \pm 0.3 \mu \mathrm{M}^{-1} \mathrm{~s}^{-1}\right)$ compared to GbXWT $\left(21.2 \pm 2.8 \mu \mathrm{M}^{-1} \mathrm{~s}^{-1}\right)$. Both rate constants are faster than the $\mathrm{O}_{2}$ rebinding to human $\mathrm{Ngb}$, Drosophila $\mathrm{Hb}$ and Arabidopsis $\mathrm{Hb}$, sugesting a high reactivity of $\mathrm{GbX}$ for oxygen molecule (Uzan et al., 2004). Photoacoustic calorimetry data show that the photo-dissociation of $\mathrm{O}_{2}$ from GbXWT leads to a small positive structure volume change $\left(0.8 \pm 12 \mathrm{~mL} \mathrm{~mol}^{-1}\right)$ but a negative structure volume change $\left(-18.2 \pm 2 \mathrm{~mL} \mathrm{~mol}^{-1}\right)$ was measured for ligand dissociation form GbXC65A. These results indicate that although the enthalpy change for $\mathrm{O}_{2}$ dissociation is not affected by the presence of the internal disulfide bridge, the volume changes are modulated by the presence of the disulfide bridge. The more negative structural volume change is also observed in Cygb and $\mathrm{Ngb}$ when the internal disulfide bridge was reduced (Astudillo, 2014). This clearly indicate that the presence 
of the internal disulfide bridge modulates overall structural changes associated with the transition from six-coordinate $\mathrm{O}_{2}$ bound $\mathrm{GbX}$ to five-coordinate deoxyGbX by $\sim 19 \mathrm{~mL}$ $\mathrm{mol}^{-1}$. In addition, the total enthalpy changes in $\mathrm{O}_{2}$ dissociation from $\mathrm{GbXC} 65 \mathrm{~A}(\Delta \mathrm{H}=$ $\left.60 \pm 3 \mathrm{kcal} \mathrm{mol}^{-1}\right)$ is similar to ligand dissociation from $\mathrm{GbXWT}(\Delta \mathrm{H}=79 \pm 30 \mathrm{kcal}$ $\left.\mathrm{mol}^{-1}\right)$. 


\section{SUMMARY}

The results presented here clearly indicate a distinct mechanism of hexacoordinate globin interactions with diatomic ligands. Specifically, we show that globin $\mathrm{X}$ has very low affinity for $\mathrm{CN}$ - in the met form and for $\mathrm{CO}$ in the deoxy form. The low affinity for $\mathrm{CO}$ is achieved by facilitating $\mathrm{CO}$ escape from the protein matrix. Interestingly, the affinity for the oxygen is significantly enhanced in GbX compare to other vertebrate globins such as Ngb and Cygb. Also, this protein exhibits an unusual stability towards $\mathrm{pH}$ unfolding as the heme group reminds attached to the apoprotein at acidic $\mathrm{pH}$. These results indicate that GbX may have physiological function(s) that is distinct from other hexa-coordinate vertebrate globins, such as Ngb and Cygb. Interestingly, the high affinity for $\mathrm{O}_{2}$ and low affinity for $\mathrm{CO}$ and $\mathrm{CN}^{-}$ligands are similar to hexa-coordinate globins found in plants, suggesting that GbX may represent a link between plant and vertebrate globins. Also, fluorescent studies of ZnPPIX reconstituted $\mathrm{Ngb}$ and Cygb revealed distinct properties of the heme binding pocket in $\mathrm{Ngb}$ and Cygb, although both proteins exhibit an increased heterogeneity of the heme binding pocket that may be characteristic feature of the hexa-coordinate globins. 


\section{LIST OF REFERENCES}

Abbruzzetti, S., Viappiani, C., Murgida, D. H., Erra-Balsells, R., \& Bilmes, G. M. (1999). Non-toxic, water-soluble photocalorimetric reference compounds for UV and visible excitation. Chemical Physics Letters, 304(3-4), 167-172. https://doi.org/10.1016/s0009-2614(99)00306-1

ALBANI, J., \& ALPERT, B. (1987). Fluctuation domains in myoglobin. Fluorescence quenching studies. European Journal of Biochemistry, 162(1), 175-178. https://doi.org/10.1111/j.1432-1033.1987.tb10558.x

Anderson, J. L. R., \& Chapman, S. K. (2005). Ligand probes for heme proteins. Dalton Transactions, $O(1), 13$. https://doi.org/10.1039/b413046d

Aono, S., Ohtaka, A., \& Okura, I. (1995). The effect of chemical modification of zinc myoglobin on the photoinduced electron transfer with methyl viologen. Journal of Molecular Catalysis A: Chemical, 96(1), 87-92. https://doi.org/10.1016/13811169(94)00032-8

Arcovito, A., Moschetti, T., D’Angelo, P., Mancini, G., Vallone, B., Brunori, M., \& Longa, S. D. (2008). An X-ray diffraction and X-ray absorption spectroscopy joint study of neuroglobin. Archives of Biochemistry and Biophysics, 475(1), 7-13. https://doi.org/10.1016/j.abb.2008.03.026

Asahina, K., Kawada, N., Kristensen, D. B., Nakatani, K., Seki, S., Shiokawa, M., Tateno, C., Obara, M., \& Yoshizato, K. (2002). Characterization of human stellate cell activation-associated protein and its expression in human liver. Biochimica et Biophysica Acta (BBA) - Gene Structure and Expression, 1577(3), 471-475. https://doi.org/10.1016/s0167-4781(02)00477-3

Astudillo, L. (2014). Conformational Dynamics Associated with Ligand Binding to Vertebrate Hexa-coordinate Hemoglobins. https://doi.org/10.25148/etd.fi14040865 
Astudillo, L., Bernad, S., Derrien, V., Sebban, P., \& Miksovska, J. (2010). Probing the role of the internal disulfide bond in regulating conformational dynamics in neuroglobin. Biophysical Journal, 99(2), L16-8. https://doi.org/10.1016/j.bpj.2010.04.033

Astudillo, L., Bernad, S., Derrien, V., Sebban, P., \& Miksovska, J. (2012). Conformational dynamics in human neuroglobin: effect of His64, Val68, and Cys120 on ligand migration. Biochemistry, 51(50), 9984-9994. https://doi.org/10.1021/bi301016u

Astudillo, L., Bernad, S., Derrien, V., Sebban, P., \& Miksovska, J. (2013). Reduction of the internal disulfide bond between Cys 38 and 83 switches the ligand migration pathway in cytoglobin. Journal of Inorganic Biochemistry, 129, 23-29. https://doi.org/10.1016/j.jinorgbio.2013.08.006

Atkins, P., \& Paula, J. de. (2010). Atkins' Physical Chemistry. OUP Oxford. https://books.google.com/books?id=BV6cAQAAQBAJ

Barboy, N., \& Feitelson, J. (1987). Quenching of the zinc-protoporphyrin triplet state as a measure of small-molecule diffusion through the structure of myoglobin. Biochemistry, 26(11), 3240-3244. https://doi.org/10.1021/bi00385a046

Beckerson, P., Svistunenko, D., \& Reeder, B. (2015). Effect of the distal histidine on the peroxidatic activity of monomeric cytoglobin. F1000Research, 4, 87. https://doi.org/10.12688/f1000research.5971.1

Beckerson, P., Wilson, M. T., Svistunenko, D. A., \& Reeder, B. J. (2015). Cytoglobin ligand binding regulated by changing haem-co-ordination in response to intramolecular disulfide bond formation and lipid interaction. The Biochemical Journal, 465(1), 127137. https://doi.org/10.1042/bj20140827

Belogortseva, N., Rubio, M., Terrell, W., \& Mikšovská, J. (2007). The contribution of heme propionate groups to the conformational dynamics associated with $\mathrm{CO}$ 
photodissociation from horse heart myoglobin. Journal of Inorganic Biochemistry, 101(7), 977-986. https://doi.org/10.1016/j.jinorgbio.2007.03.009

Bholah, T. C., Neergheen-Bhujun, V. S., Hodges, N. J., Dyall, S. D., \& Bahorun, T. (2015). Cytoglobin as a Biomarker in Cancer: Potential Perspective for Diagnosis and Management. BioMed Research International, 2015, 824514. https://doi.org/10.1155/2015/824514

Birukou, I., Schweers, R. L., \& Olson, J. S. (2010). Distal histidine stabilizes bound O2 and acts as a gate for ligand entry in both subunits of adult human hemoglobin. The Journal of Biological Chemistry, 285(12), 8840-8854. https://doi.org/10.1074/jbc.m109.053934

Blank, M., \& Burmester, T. (2012). Widespread occurrence of N-terminal acylation in animal globins and possible origin of respiratory globins from a membrane-bound ancestor. Molecular Biology and Evolution, 29(11), 3553-3561. https://doi.org/10.1093/molbev/mss164

Blank, M., Kiger, L., Thielebein, A., Gerlach, F., Hankeln, T., Marden, M. C., \& Burmester, T. (2011). Oxygen supply from the bird's eye perspective: globin E is a respiratory protein in the chicken retina. The Journal of Biological Chemistry, 286(30), 26507-26515. https://doi.org/10.1074/jbc.m111.224634

Blank, M., Wollberg, J., Gerlach, F., Reimann, K., Roesner, A., Hankeln, T., Fago, A., Weber, R. E., \& Burmester, T. (2011). A membrane-bound vertebrate globin. PloS One, 6(9), e25292. https://doi.org/10.1371/journal.pone.0025292

Bocahut, A., Derrien, V., Bernad, S., Sebban, P., Sacquin-Mora, S., Guittet, E., \& Lescop, E. (2012). Heme orientation modulates histidine dissociation and ligand binding kinetics in the hexacoordinated human neuroglobin. Journal of Biological Inorganic Chemistry: JBIC: A Publication of the Society of Biological Inorganic Chemistry, 18(1), 111-122. https://doi.org/10.1007/s00775-012-0956-2 
Burmester, T, \& Hankeln, T. (2009). What is the function of neuroglobin? Journal of Experimental Biology, 212(10), 1423-1428. https://doi.org/10.1242/jeb.000729

Burmester, T, \& Hankeln, T. (2014). Function and evolution of vertebrate globins. Acta Physiologica, 211(3), 501-514. https://doi.org/10.1111/apha.12312

Burmester, Thorsten, Ebner, B., Weich, B., \& Hankeln, T. (2002). Cytoglobin: A Novel Globin Type Ubiquitously Expressed inVertebrate Tissues. Molecular Biology and Evolution, 19(4), 416-421. https://doi.org/10.1093/oxfordjournals.molbev.a004096

Burmester, Thorsten, Weich, B., Reinhardt, S., \& Hankeln, T. (2000). A vertebrate globin expressed in the brain. Nature, 407(6803), 520-523. https://doi.org/10.1038/35035093

Butcher, D., Bernad, S., Derrien, V., Sebban, P., \& Miksovska, J. (2017). Role of Ionic Strength and $\mathrm{pH}$ in Modulating Thermodynamic Profiles Associated with CO Escape from Rice Nonsymbiotic Hemoglobin 1. The Journal of Physical Chemistry. B, 121(2), 351-364. https://doi.org/10.1021/acs.jpcb.6b06933

Chandra, R., Tiwari, M., Kaur, P., Sharma, M., Jain, R., \& Dass, S. (2000). Metalloporphyrins-Applications and clinical significance. Indian Journal of Clinical Biochemistry : IJCB, 15(Suppl 1), 183-199. https://doi.org/10.1007/bf02867558

Corti, P., Xue, J., Tejero, J., Wajih, N., Sun, M., Stolz, D. B., Tsang, M., Kim-Shapiro, D. B., \& Gladwin, M. T. (2016). Globin X is a six-coordinate globin that reduces nitrite to nitric oxide in fish red blood cells. Proceedings of the National Academy of Sciences of the United States of America, 113(30), 8538-8543. https://doi.org/10.1073/pnas.1522670113

Dewilde, S., Kiger, L., Burmester, T., Hankeln, T., Baudin-Creuza, V., Aerts, T., Marden, M. C., Caubergs, R., \& Moens, L. (2001). Biochemical Characterization and Ligand Binding Properties of Neuroglobin, a Novel Member of the Globin Family. Journal of Biological Chemistry, 276(42), 38949-38955. https://doi.org/10.1074/jbc.m106438200 
Dou, Y., Olson, J. S., Wilkinson, A. J., \& Ikeda-Saito, M. (1996). Mechanism of Hydrogen Cyanide Binding to Myoglobin $\uparrow$. Biochemistry, 35(22), 7107-7113. https://doi.org/10.1021/bi9600299

Drew, E. D., Mavridis, L., \& Janes, R. W. (2018). PDB2CD: A Web-Based Application for the Generation of Circular Dichroism Spectra from Protein Atomic Coordinates. Biophysical Journal, 114(3), 46a. https://doi.org/10.1016/j.bpj.2017.11.302

Dröge, J., \& Makałowski, W. (2011). Phylogenetic analysis reveals wide distribution of globin X. Biology Direct, 6(1), 54. https://doi.org/10.1186/1745-6150-6-54

Du, W., Syvitski, R., Dewilde, S., Moens, L., \& Mar, G. N. L. (2003). Solution 1h NMR characterization of equilibrium heme orientational disorder with functional consequences in mouse neuroglobin. Journal of the American Chemical Society, 125(27), 8080-8081. https://doi.org/10.1021/ja034584r

Exertier, C., Milazzo, L., Freda, I., Montemiglio, L. C., Scaglione, A., Cerutti, G., Parisi, G., Anselmi, M., Smulevich, G., Savino, C., \& Vallone, B. (2019). Proximal and distal control for ligand binding in neuroglobin: role of the CD loop and evidence for His64 gating. Scientific Reports, 9(1), 5326. https://doi.org/10.1038/s41598-01941780-3

Fago, A., Mathews, A. J., Moens, L., Dewilde, S., \& Brittain, T. (2006). The reaction of neuroglobin with potential redox protein partners cytochrome b 5 and cytochrome $\mathrm{c}$. FEBS Letters, 580(20), 4884-4888. https://doi.org/10.1016/j.febslet.2006.08.003

Fanelli, A. R., Antonini, E., \& Caputo, A. (1958). Studies on the structure of hemoglobin I. Physicochemical properties of human globin. Biochimica et Biophysica Acta, 30(3), 608-615. https://doi.org/10.1016/0006-3002(58)90108-2

Flonta, S. E., Arena, S., Pisacane, A., Michieli, P., \& Bardelli, A. (2009). Expression and functional regulation of myoglobin in epithelial cancers. The American Journal of Pathology, 175(1), 201-206. https://doi.org/10.2353/ajpath.2009.081124 
Fuchs, C., Burmester, T., \& Hankeln, T. (2006). The amphibian globin gene repertoire as revealed by the Xenopus genome. Cytogenetic and Genome Research, 112(3-4), 296-306. https://doi.org/10.1159/000089884

Garry, D. J., Ordway, G. A., Lorenz, J. N., Radford, N. B., Chin, E. R., Grange, R. W., Bassel-Duby, R., \& Williams, R. S. (1998). Mice without myoglobin. Nature, 395(6705), 905-908. https://doi.org/10.1038/27681

Gensch, T., \& Viappiani, C. (2003). Time-resolved photothermal methods: accessing time-resolved thermodynamics of photoinduced processes in chemistry and biologyDedicated to Professor Silvia Braslavsky, to mark her great contribution to photochemistry and photobiology particularly in the field of photothermal methods. $\begin{array}{lllll}\text { Photochemical \& Photobiological } & \text { Sciences, } & 2(7), & 699 .\end{array}$ https://doi.org/10.1039/b303177b

Gibson, Q. H. (1956). An apparatus for flash photolysis and its application to the reactions of myoglobin with gases. The Journal of Physiology, 134(1), 112-122. https://doi.org/10.1113/jphysiol.1956.sp005627

Gorr, T. A., Wichmann, D., Pilarsky, C., Theurillat, J.-P., Fabrizius, A., Laufs, T., Bauer, T., Koslowski, M., Horn, S., Burmester, T., Hankeln, T., \& Kristiansen, G. (2010). Old proteins - new locations: myoglobin, haemoglobin, neuroglobin and cytoglobin in solid tumours and cancer cells. Acta Physiologica (Oxford, England), 202(3), 563-581. https://doi.org/10.1111/j.1748-1716.2010.02205.x

Guo, X., Philipsen, S., \& Tan-Un, K.-C. (2007). Study of the hypoxia-dependent regulation of human CYGB gene. Biochemical and Biophysical Research Communications, 364(1), 145-150. https://doi.org/10.1016/j.bbrc.2007.09.108

Hamdane, D., Kiger, L., Dewilde, S., Green, B. N., Pesce, A., Uzan, J., Burmester, T., Hankeln, T., Bolognesi, M., Moens, L., \& Marden, M. C. (2003). The Redox State of the Cell Regulates the Ligand Binding Affinity of Human Neuroglobin and Cytoglobin. Journal of Biological Chemistry, 278(51), 51713-51721. https://doi.org/10.1074/jbc.m309396200 
Hamdane, D., Kiger, L., Dewilde, S., Uzan, J., Burmester, T., Hankeln, T., Moens, L., \& Marden, M. C. (2005). Hyperthermal stability of neuroglobin and cytoglobin: Thermal stability of neuroglobin. FEBS Journal, 272(8), 2076-2084. https://doi.org/10.1111/j.1742-4658.2005.04635.x

Hanai, S., Tsujino, H., Yamashita, T., Torii, R., Sawai, H., Shiro, Y., Oohora, K., Hayashi, T., \& Uno, T. (2017). Roles of N- and C-terminal domains in the ligandbinding properties of cytoglobin. Journal of Inorganic Biochemistry, 179, 1-9. https://doi.org/10.1016/j.jinorgbio.2017.11.003

HARRISON, S. C., \& BLOUT, E. R. (1965). REVERSIBLE CONFORMATIONAL CHANGES OF MYOGLOBIN AND APOMYOGLOBIN. The Journal of Biological Chemistry, 240, 299-303.

Hartridge, H., \& Roughton, F. J. W. (1923). The Kinetics of Hœmoglobin. II. The Velocity with Which Oxygen Dissociates from Its Combination with Hœmoglobin. JSTOR, 104(726), 395-430. www.jstor.org/stable/94180

Hayashi, T., Matsuo, T., Hitomi, Y., Okawa, K., Suzuki, A., Shiro, Y., Iizuka, T., Hisaeda, Y., \& Ogoshi, H. (2002). Contribution of heme-propionate side chains to structure and function of myoglobin: chemical approach by artificially created prosthetic groups. Journal of Inorganic Biochemistry, 91(1), 94-100. https://doi.org/10.1016/s0162-0134(02)00423-3

Hendgen-Cotta, U. B., Merx, M. W., Shiva, S., Schmitz, J., Becher, S., Klare, J. P., Steinhoff, H.-J., Goedecke, A., Schrader, J., Gladwin, M. T., Kelm, M., \& Rassaf, T. (2008). Nitrite reductase activity of myoglobin regulates respiration and cellular viability in myocardial ischemia-reperfusion injury. Proceedings of the National Academy of Sciences of the United States of America, 105(29), 10256-10261. https://doi.org/10.1073/pnas.0801336105

Holde, K. E. V., Johnson, W. C., \& Ho, P. S. (1998). Principles of Physical Biochemistry. Prentice Hall. https://books.google.com/books?id=TrPwAAAAMAAJ 
Hoogewijs, D., Ebner, B., Germani, F., Hoffmann, F. G., Fabrizius, A., Moens, L., Burmester, T., Dewilde, S., Storz, J. F., Vinogradov, S. N., \& Hankeln, T. (2011). Androglobin: a chimeric globin in metazoans that is preferentially expressed in Mammalian testes. Molecular Biology and Evolution, 29(4), 1105-1114. https://doi.org/10.1093/molbev/msr246

Hou, S., Larsen, R. W., Boudko, D., Riley, C. W., Karatan, E., Zimmer, M., Ordal, G. W., \& Alam, M. (2000). Myoglobin-like aerotaxis transducers in Archaea and Bacteria. Nature, 403(6769), 540-544. https://doi.org/10.1038/35000570

Kakar, S., Hoffman, F. G., Storz, J. F., Fabian, M., \& Hargrove, M. S. (2010). Structure and reactivity of hexacoordinate hemoglobins. Biophysical Chemistry, 152(1-3), 1-14. https://doi.org/10.1016/j.bpc.2010.08.008

Karp, D. A., Gittis, A. G., Stahley, M. R., Fitch, C. A., Stites, W. E., \& E, B. G.-M. (2007). High Apparent Dielectric Constant Inside a Protein Reflects Structural Reorganization Coupled to the Ionization of an Internal Asp. Biophysical Journal, 92(6), 2041-2053. https://doi.org/10.1529/biophysj.106.090266

Kelly, S. M., Jess, T. J., \& Price, N. C. (2005). How to study proteins by circular dichroism. Biochimica et Biophysica Acta (BBA) - Proteins and Proteomics, 1751(2), 119-139. https://doi.org/10.1016/j.bbapap.2005.06.005

Khan, A. A., Wang, Y., Sun, Y., Mao, X. O., Xie, L., Miles, E., Graboski, J., Chen, S., Ellerby, L. M., Jin, K., \& Greenberg, D. A. (2006). Neuroglobin-overexpressing transgenic mice are resistant to cerebral and myocardial ischemia. Proceedings of the National Academy of Sciences, 103(47), 17944-17948. https://doi.org/10.1073/pnas.0607497103

Klostermeier, D., \& Rudolph, M. G. (2018). Biophysical Chemistry. CRC Press. https://books.google.com/books?id=ZchwDwAAQBAJ 
Koch, J., \& Burmester, T. (2016). Membrane-bound globin X protects the cell from reactive oxygen species. Biochemical and Biophysical Research Communications, 469(2), 275-280. https://doi.org/10.1016/j.bbrc.2015.11.105

Lakowicz, J. R. (2007). Principles of Fluorescence Spectroscopy. Springer US. https://books.google.com/books?id=-PSybuLNxcAC

Lakowicz, J. R., Laczko, G., Cherek, H., Gratton, E., \& Limkeman, M. (1984). Analysis of fluorescence decay kinetics from variable-frequency phase shift and modulation data. Biophysical Journal, 46(4), 463-477. https://doi.org/10.1016/s00063495(84)84043-6

Larsen, R. W., \& Mikšovská, J. (2007). Time resolved thermodynamics of ligand binding to heme proteins. Coordination Chemistry Reviews, 251(9-10), 1101-1127. https://doi.org/10.1016/j.ccr.2006.08.018

Laufs, T. L., Wystub, S., Reuss, S., Burmester, T., Saaler-Reinhardt, S., \& Hankeln, T. (2004). Neuron-specific expression of neuroglobin in mammals. Neuroscience Letters, 362(2), 83-86. https://doi.org/10.1016/j.neulet.2004.02.072

Lechauve, C., Rezaei, H., Celier, C., Kiger, L., Corral-Debrinski, M., Noinville, S., Chauvierre, C., Hamdane, D., Pato, C., \& Marden, M. C. (2009). Neuroglobin and prion cellular localization: investigation of a potential interaction. Journal of Molecular Biology, 388(5), 968-977. https://doi.org/10.1016/j.jmb.2009.03.047

Li, H., Hemann, C., Abdelghany, T. M., El-Mahdy, M. A., \& Zweier, J. L. (2012). Characterization of the mechanism and magnitude of cytoglobin-mediated nitrite reduction and nitric oxide generation under anaerobic conditions. The Journal of Biological Chemistry, 287(43), 36623-36633. https://doi.org/10.1074/jbc.m112.342378

Liang, Z.-X., Nocek, J. M., Huang, K., Hayes, R. T., Kurnikov, I. V., Beratan, D. N., \& Hoffman, B. M. (2002). Dynamic Docking and Electron Transfer between Zn- 
myoglobin and Cytochrome b 5. Journal of the American Chemical Society, 124(24), 6849-6859. https://doi.org/10.1021/ja0127032

Liu, H.-X., Li, L., Yang, X.-Z., Wei, C.-W., Cheng, H.-M., Gao, S.-Q., Wen, G.-B., \& Lin, Y.-W. (2019). Enhancement of protein stability by an additional disulfide bond designed in human neuroglobin. RSC Advances, 9(8), 4172-4179. https://doi.org/10.1039/c8ra10390a

Liu, J., Yu, Z., Guo, S., Lee, S.-R., Xing, C., Zhang, C., Gao, Y., Nicholls, D. G., Lo, E. H., \& Wang, X. (2009). Effects of neuroglobin overexpression on mitochondrial function and oxidative stress following hypoxia/reoxygenation in cultured neurons. Journal of Neuroscience Research, 87(1), 164-170. https://doi.org/10.1002/jnr.21826

Liu, X., El-Mahdy, M. A., Boslett, J., Varadharaj, S., Hemann, C., Abdelghany, T. M., Ismail, R. S., Little, S. C., Zhou, D., Thuy, L. T. T., Kawada, N., \& Zweier, J. L. (2017). Cytoglobin regulates blood pressure and vascular tone through nitric oxide metabolism in the vascular wall. Nature Communications, 8(1), 14807. https://doi.org/10.1038/ncomms14807

Livesey, A. K., \& Brochon, J. C. (1987). Analyzing the Distribution of Decay Constants in Pulse-Fluorimetry Using the Maximum Entropy Method. Biophysical Journal, 52(5), 693-706. https://doi.org/10.1016/s0006-3495(87)83264-2

Mach, H., Middaugh, C. R., \& Lewis, R. V. (1992). Statistical determination of the average values of the extinction coefficients of tryptophan and tyrosine in native proteins. Analytical Biochemistry, 200(1), 74-80. https://doi.org/10.1016/00032697(92)90279-g

Makino, M., Sawai, H., Shiro, Y., \& Sugimoto, H. (2011). Crystal structure of the carbon monoxide complex of human cytoglobin. Proteins, 79(4), 1143-1153. https://doi.org/10.1002/prot.22950

Makino, M., Sugimoto, H., Sawai, H., Kawada, N., Yoshizato, K., \& Shiro, Y. (2006). High-resolution structure of human cytoglobin: identification of extra N-and C-termini 
and a new dimerization mode. Acta Crystallographica Section D Biological Crystallography, 62(6), 671-677. https://doi.org/10.1107/s0907444906013813

Mathai, C., Jourd'heuil, F. L., Lopez-Soler, R. I., \& Jourd'heuil, D. (2020). Emerging perspectives on cytoglobin, beyond NO dioxygenase and peroxidase. Redox Biology, 32, 101468. https://doi.org/10.1016/j.redox.2020.101468

McRonald, F. E., Liloglou, T., Xinarianos, G., Hill, L., Rowbottom, L., Langan, J. E., Ellis, A., Shaw, J. M., Field, J. K., \& Risk, J. M. (2006). Down-regulation of the cytoglobin gene, located on 17q25, in tylosis with oesophageal cancer (TOC): evidence for trans-allele repression. Human Molecular Genetics, 15(8), 1271-1277. https://doi.org/10.1093/hmg/dd1042

Mikšovská, J., Day, J. H., \& Larsen, R. W. (2003). Volume and enthalpy profiles of CO rebinding to horse heart myoglobin. JBIC Journal of Biological Inorganic Chemistry, 8(6), 621-625. https://doi.org/10.1007/s00775-003-0457-4

Mikšovská, J., Horsa, S., Davis, M. F., \& Franzen, S. (2008). Conformational Dynamics Associated with Photodissociation of $\mathrm{CO}$ from Dehaloperoxidase Studied Using Photoacoustic Calorimetry $\dagger$. Biochemistry, 47(44), 11510-11517. https://doi.org/10.1021/bi8012033

Moore, J. C., Battino, R., Rettich, T. R., Handa, Y. P., \& Wilhelm, E. (1982). Partial molar volumbes of gases at infinite dilution in water at $298.15 \mathrm{~K}$. Journal of Chemical \& Engineering Data, 27(1), 22-24. https://doi.org/10.1021/je00027a005

Naito, N. R., Huang, H., Sturgess, A. W., Nocek, J. M., \& Hoffman, B. M. (1998). Binding and Electron Transfer between Cytochrome $b 5$ and the Hemoglobin $\alpha$ - and $\beta$ Subunits through the Use of [Zn, Fe] Hybrids. Journal of the American Chemical Society, 120(44), 11256-11262. https://doi.org/10.1021/ja982009v

Nakatani, K., Okuyama, H., Shimahara, Y., Saeki, S., Kim, D.-H., Nakajima, Y., Seki, S., Kawada, N., \& Yoshizato, K. (2003). Cytoglobin/STAP, its unique localization in 
splanchnic fibroblast-like cells and function in organ fibrogenesis. Laboratory Investigation, 84(1), 91-101. https://doi.org/10.1038/labinvest.3700013

Nienhaus, G. U. (2010). Protein'Ligand Interactions: Methods and Applications. Humana Press. https://books.google.com/books?id=2RatcQAACAAJ

Oleksiewicz, U., Liloglou, T., Field, J. K., \& Xinarianos, G. (2011). Cytoglobin: biochemical, functional and clinical perspective of the newest member of the globin family. Cellular and Molecular Life Sciences: CMLS, 68(23), 3869-3883. https://doi.org/10.1007/s00018-011-0764-9

Olson, J. S., Mathews, A. J., Rohlfs, R. J., Springer, B. A., Egeberg, K. D., Sligar, S. G., Tame, J., Renaud, J.-P., \& Nagai, K. (1988). The role of the distal histidine in myoglobin and haemoglobin. Nature, 336(6196), 265-266. https://doi.org/10.1038/336265a0

Peters, K. S., Watson, T., \& Logan, T. (1992). Photoacoustic calorimetry study of human carboxyhemoglobin. Journal of the American Chemical Society, 114(11), 4276-4278. https://doi.org/10.1021/ja00037a035

Picotti, P., Dewilde, S., Fago, A., Hundahl, C., Filippis, V. D., Moens, L., \& Fontana, A. (2009). Unusual stability of human neuroglobin at low $\mathrm{pH}$ - molecular mechanisms and biological significance: Unusual acid stability of human neuroglobin. FEBS Journal, 276(23), 7027-7039. https://doi.org/10.1111/j.1742-4658.2009.07416.x

Poulos, T. L. (2014). Heme enzyme structure and function. Chemical Reviews, 114(7), 3919-3962. https://doi.org/10.1021/cr400415k

Reuss, S., Saaler-Reinhardt, S., Weich, B., Wystub, S., Reuss, M. H., Burmester, T., \& Hankeln, T. (2002). Expression analysis of neuroglobin mRNA in rodent tissues. Neuroscience, 115(3), 645-656. https://doi.org/10.1016/s0306-4522(02)00536-5 
Roesner, A., Fuchs, C., Hankeln, T., \& Burmester, T. (2004). A Globin Gene of Ancient Evolutionary Origin in Lower Vertebrates: Evidence for Two Distinct Globin Families in Animals. Molecular Biology and Evolution, 22(1), 12-20. https://doi.org/10.1093/molbev/msh258

Rohlfs, R. J., Mathews, A. J., Carver, T. E., Olson, J. S., Springer, B. A., Egeberg, K. D., \& Sligar, S. G. (1990). The effects of amino acid substitution at position E7 (residue 64) on the kinetics of ligand binding to sperm whale myoglobin. The Journal of Biological Chemistry, 265(6), 3168-3176.

Rosenheck, K., \& Doty, P. (1961). THE FAR ULTRAVIOLET ABSORPTION SPECTRA OF POLYPEPTIDE AND PROTEIN SOLUTIONS AND THEIR DEPENDENCE ON CONFORMATION. Proceedings of the National Academy of Sciences, 47(11), 1775-1785. https://doi.org/10.1073/pnas.47.11.1775

Ross, J. A., \& Jameson, D. M. (2008). Time-resolved methods in biophysics. 8. Frequency domain fluorometry: applications to intrinsic protein fluorescence. Photochemical \& Photobiological Sciences: Official Journal of the European Photochemistry Association and the European Society for Photobiology, 7(11), 13011312. https://doi.org/10.1039/b804450n

Sawai, H., Kawada, N., Yoshizato, K., Nakajima, H., Aono, S., \& Shiro, Y. (2003). Characterization of the Heme Environmental Structure of Cytoglobin, a Fourth Globin in Humans $\uparrow$. Biochemistry, 42(17), 5133-5142. https://doi.org/10.1021/bi027067e

Schaberle, F. A., Nunes, R. M. D., Barroso, M., Serpa, C., \& Arnaut, L. G. (2010). Analytical solution for time-resolved photoacoustic calorimetry data and applications to two typical photoreactions. Photochemical \& Photobiological Sciences: Official Journal of the European Photochemistry Association and the European Society for Photobiology, 9(6), 812-822. https://doi.org/10.1039/c0pp00025f

Schuresko, D. D., \& Webb, W. W. (1978). Carboxylation kinetics of hemoglobin and myoglobin: linear transient response to step perturbation by laser photolysis. Biophysical Journal, 24(1), 382-383. https://doi.org/10.1016/s0006-3495(78)85390-9 
Sheftel, A. D., Mason, A. B., \& Ponka, P. (2011). The long history of iron in the Universe and in health and disease. Biochimica et Biophysica Acta, 1820(3), 161-187. https://doi.org/10.1016/j.bbagen.2011.08.002

Shivapurkar, N., Stastny, V., Okumura, N., Girard, L., Xie, Y., Prinsen, C., Thunnissen, F. B., Wistuba, I. I., Czerniak, B., Frenkel, E., Roth, J. A., Liloglou, T., Xinarianos, G., Field, J. K., Minna, J. D., \& Gazdar, A. F. (2008). Cytoglobin, the newest member of the globin family, functions as a tumor suppressor gene. Cancer Research, 68(18), 7448-7456. https://doi.org/10.1158/0008-5472.can-08-0565

Singh, S., Manda, S. M., Sikder, D., Birrer, M. J., Rothermel, B. A., Garry, D. J., \& Mammen, P. P. A. (2009). Calcineurin activates cytoglobin transcription in hypoxic myocytes. The Journal of Biological Chemistry, 284(16), 10409-10421. https://doi.org/10.1074/jbc.m809572200

Smagghe, B. J., Sarath, G., Ross, E., Hilbert, J., \& Hargrove, M. S. (2006). Slow Ligand Binding Kinetics Dominate Ferrous Hexacoordinate Hemoglobin Reactivities and Reveal Differences between Plants and Other Species $\uparrow$. Biochemistry, 45(2), 561-570. https://doi.org/10.1021/bi0519021

Steinbach, Peter J. (2012). Filtering artifacts from lifetime distributions when maximizing entropy using a bootstrapped model. Analytical Biochemistry, 427(1), 102105. https://doi.org/10.1016/j.ab.2012.04.008

Steinbach, Peter J., Ionescu, R., \& Matthews, C. R. (2002). Analysis of Kinetics Using a Hybrid Maximum-Entropy/Nonlinear-Least-Squares Method: Application to Protein Folding. Biophysical Journal, 82(4), 2244-2255. https://doi.org/10.1016/s00063495(02)75570-7

Steinbach, P.J. (1996). Two-dimensional distributions of activation enthalpy and entropy from kinetics by the maximum entropy method. Biophysical Journal, 70(3), 1521-1528. https://doi.org/10.1016/s0006-3495(96)79714-x 
Steinbach, P.J., Chu, K., Frauenfelder, H., Johnson, J. B., Lamb, D. C., Nienhaus, G. U., Sauke, T. B., \& Young, R. D. (1992). Determination of rate distributions from kinetic experiments. Biophysical Journal, 61(1), 235-245. https://doi.org/10.1016/s0006-3495(92)81830-1

Storz, J. F., \& Moriyama, H. (2008). Mechanisms of hemoglobin adaptation to high altitude hypoxia. High Altitude Medicine \& Biology, 9(2), 148-157. https://doi.org/10.1089/ham.2007.1079

Tangar, A., Derrien, V., Lei, R., Estevez, M. J. S., Sebban, P., Bernad, S., \& Miksovska, J. (2019). Utility of fluorescent heme analogue ZnPPIX to monitor conformational heterogeneity in vertebrate hexa-coordinated globins. Metallomics, 11(5), 906-913. https://doi.org/10.1039/c8mt00332g

Teale, F. W. J. (1959). Cleavage of the haem-protein link by acid methylethylketone. Biochimica et Biophysica Acta, 35, 543. https://doi.org/10.1016/0006-3002(59)90407$\mathrm{X}$

Tejero, J., Sparacino-Watkins, C. E., Ragireddy, V., Frizzell, S., \& Gladwin, M. T. (2015). Exploring the mechanisms of the reductase activity of neuroglobin by sitedirected mutagenesis of the heme distal pocket. Biochemistry, 54(3), 722-733. https://doi.org/10.1021/bi501196k

Thuy, L. T. T., Thuy, T. T. V., Matsumoto, Y., Hai, H., Ikura, Y., Yoshizato, K., \& Kawada, N. (2016). Absence of cytoglobin promotes multiple organ abnormalities in aged mice. Scientific Reports, 6(1), 24990. https://doi.org/10.1038/srep24990

Totzeck, M., Hendgen-Cotta, U. B., Luedike, P., Berenbrink, M., Klare, J. P., Steinhoff, H.-J., Semmler, D., Shiva, S., Williams, D., Kipar, A., Gladwin, M. T., Schrader, J., Kelm, M., Cossins, A. R., \& Rassaf, T. (2012). Nitrite regulates hypoxic vasodilation via myoglobin-dependent nitric oxide generation. Circulation, 126(3), 325-334. https://doi.org/10.1161/circulationaha.111.087155 
Trent, J. T., \& Hargrove, M. S. (2002). A Ubiquitously Expressed Human Hexacoordinate Hemoglobin. Journal of Biological Chemistry, 277(22), 19538-19545. https://doi.org/10.1074/jbc.m201934200

Tsujino, H., Yamashita, T., Nose, A., Kukino, K., Sawai, H., Shiro, Y., \& Uno, T. (2014). Disulfide bonds regulate binding of exogenous ligand to human cytoglobin. Journal of Inorganic Biochemistry, 135, 20-27. https://doi.org/10.1016/j.jinorgbio.2014.02.011

Uddin, R. G. E.-J. (2012). The Use of Spectrophotometry UV-Vis for the Study of Porphyrins (p. Ch. 6). IntechOpen. https://doi.org/10.5772/38797

Uzan, J., Dewilde, S., Burmester, T., Hankeln, T., Moens, L., Hamdane, D., Marden, M. C., \& Kiger, L. (2004). Neuroglobin and Other Hexacoordinated Hemoglobins Show a Weak Temperature Dependence of Oxygen Binding. Biophysical Journal, 87(2), 1196-1204. https://doi.org/10.1529/biophysj.104.042168

Valeur, B. (2001). Molecular Fluorescence. 155-199. https://doi.org/10.1002/3527600248.ch6

Vallone, B., Nienhaus, K., Matthes, A., Brunori, M., \& Nienhaus, G. U. (2004). The structure of carbonmonoxy neuroglobin reveals a heme-sliding mechanism for control of ligand affinity. Proceedings of the National Academy of Sciences, 101(50), 1735117356. https://doi.org/10.1073/pnas.0407633101

Vetromile, C. M., Miksovska, J., \& Larsen, R. W. (2011). Time resolved thermodynamics associated with ligand photorelease in heme peroxidases and globins: Open access channels versus gated ligand release. Biochimica et Biophysica Acta, 1814(8), 1065-1076. https://doi.org/10.1016/j.bbapap.2011.01.009

Voet, D., \& Voet, J. G. (2010). Biochemistry, 4th Edition. J. Wiley \& Sons.

Wakasugi, K., Nakano, T., Kitatsuji, C., \& Morishima, I. (2004). Human neuroglobin interacts with flotillin-1, a lipid raft microdomain-associated protein. Biochemical and 
Biophysical Research Communications, 318(2), 453-460. https://doi.org/10.1016/j.bbrc.2004.04.045

Wakasugi, K., Nakano, T., \& Morishima, I. (2003). Oxidized Human Neuroglobin Acts as a Heterotrimeric $\mathrm{G} \alpha$ Protein Guanine Nucleotide Dissociation Inhibitor. Journal of Biological Chemistry, 278(38), 36505-36512. https://doi.org/10.1074/jbc.m305519200

Wakasugi, K., Nakano, T., \& Morishima, I. (2004). Association of Human Neuroglobin with Cystatin C, a Cysteine Proteinase Inhibitor $\dagger$. Biochemistry, 43(18), 5119-5125. https://doi.org/10.1021/bi0495782

Wang, W.-H., Lu, J.-X., Yao, P., Xie, Y., \& Huang, Z.-X. (2003). The distinct heme coordination environments and heme-binding stabilities of His39Ser and His39Cys mutants of cytochrome b5. Protein Engineering Design and Selection, 16(12), 10471054. https://doi.org/10.1093/protein/gzg134

Weber, G., \& Teale, F. J. W. (1959). Electronic energy transfer in haem proteins. Discussions of the Faraday Society, 27(0), 134. https://doi.org/10.1039/df9592700134

Weinacht, T. C., \& Pearson, B. J. (2018). Time-Resolved Spectroscopy. 63-72. https://doi.org/10.1201/9780429440823-4

Worsfold, Paul. J., \& Zagatto, Elias. A. G. (2005). Spectrophotometry | Overview (P. Worsfold, A. Townshend, \& C. Poole, Eds.; p. 5000). Elsevier.

Xu, J., Li, L., Yin, G., Li, H., \& Du, W. (2009). Ligand orientation of human neuroglobin obtained from solution NMR and molecular dynamics simulation as compared with X-ray crystallography. Journal of Inorganic Biochemistry, 103(12), 1693-1701. https://doi.org/10.1016/j.jinorgbio.2009.09.016

Xu, J., Yin, G., Huang, F., Wang, B., \& Du, W. (2009). Molecular dynamics simulation of a carboxy murine neuroglobin mutated on the proximal side: heme displacement and 
concomitant rearrangement in loop regions. Journal of Molecular Modeling, 16(4), 759-770. https://doi.org/10.1007/s00894-009-0581-3

Yu, Z., Xu, J., Liu, N., Wang, Y., Li, X., Pallast, S., Leyen, K. van, \& Wang, X. (2012). Mitochondrial distribution of neuroglobin and its response to oxygen-glucose deprivation in primary-cultured mouse cortical neurons. Neuroscience, 218, 235-242. https://doi.org/10.1016/j.neuroscience.2012.05.054

Zhang, S., Li, X., Jourd'heuil, F. L., Qu, S., Devejian, N., Bennett, E., Jourd'heuil, D., \& Cai, C. (2017). Cytoglobin Promotes Cardiac Progenitor Cell Survival against Oxidative Stress via the Upregulation of the NFkB/iNOS Signal Pathway and Nitric Oxide Production. Scientific Reports, 7(1), 10754. https://doi.org/10.1038/s41598-01711342-6 


\section{VITA}

\section{RUIPENG LEI}

2014-present Ph. D. student (Biochemistry) Florida International University, FL (GPA: 3.72)

2009-2012 B.S. (Biotechnology) Huaiyin Normal University, Huai an, Jiangsu

\section{PUBLICATIONS AND SELECTED CONFERENCE PRESENTATIONS}

Ren Y, Lai Y, Laverde EE, Lei R, Rein HL, et al. (2017) Modulation of trinucleotide repeat instability by DNA polymerase $\beta$ polymorphic variant R137Q. PLOS ONE 12(5): e0177299.

Antonija Tangar; Vale'rie Derrien; Ruipeng Lei; Maria Jose Santiago Estevez; Pierre Sebban; Sophie Bernad and Jaroslava Miksovska. "Utility of fluorescent heme analogue ZnPPIX to monitor conformational heterogeneity in vertebrate hexacoordinated globins". Metallomics. 2019 May 22;11(5):906-913. doi: $10.1039 / \mathrm{c} 8 \mathrm{mt} 00332 \mathrm{~g}$.

Wen, Zhiwei; Tuttle, Paloma; Howlader, A. Hasan; Vasilyeva, Anna; Gonzales, Laura; Tangar, Antonija; Lei, Ruipeng; Laverde, Eduardo; Liu, Yuan; Miksovska, Jaroslava; Wnuk, Stanislaw. "Fluorescent 5-Pyrimidine and 8-Purine Nucleosides Modified with N-unsubstituted 1,2,3-Triazol-4-yl Moiety”. The Journal of Organic Chemistry. 2019 Mar 15;84(6):3624-3631. doi: 10.1021/acs.joc.8b03135. Epub 2019 Mar 6.

Yanhao Lai, Ruipeng Lei*, Yaou Ren, Yuan Liu. "Methods to Study Trinucleotide Repeat Instability Induced by DNA Damage and Repair". Methods Mol Biol. 2019;1999:87-101. doi: 10.1007/978-1-4939-9500-4_5.

Judy L. Vasquez, Yanhao Lai, Thirunavukkarasu Annamalai, Zhongliang Jiang, Manqi Zhang, Ruipeng Lei, Zunzhen Zhang, Yuan Liu, Yuk-Ching Tse-Dinh, Irina U. Agoulnik. "Inhibition of base excision repair by natamycin suppresses prostate cancer cell proliferation". Biochimie. 2020 Jan;168:241-250. doi: 10.1016/j.biochi.2019.11.008. Epub 2019 Nov 19.

*Co-first author 
Jaroslava Miksovska, Ruipeng Lei, Sophie Bernad, Valerie Derrien. Distinct mechanism of oxygen and carbon monoxide interactions with heme protein. $62^{\text {nd }}$ Biophysical society meeting, Feb 17-21, 2018, San Fransisco, CA.

Jaroslava Miksovska, Ruipeng Lei, Sophie Bernad, Valerie Derrien. Distinct mechanism of oxygen and carbon monoxide interactions with heme protein. 94th Florida Annual Meeting and Exposition (FAME) conference, May 3-5, 2018, Palm Harbor, FL.

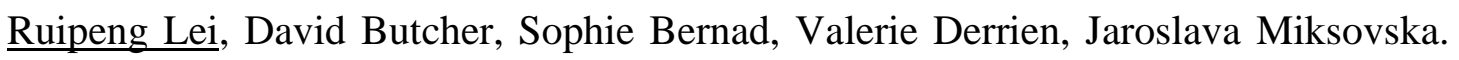
Mutation of Residues in CD Loop and Distal Pocket Impact Protein Stability of Human Neuroglobin. $63^{\text {rd }}$ Biophysical society meeting, Mar 02-06, 2019, Baltimore, MD.

Ruipeng Lei, David Butcher, Sophie Bernad, Valerie Derrien, Jaroslava Miksovska. Mutation of Residues in CD Loop and Distal Pocket Impact Protein Stability of Human Neuroglobin. ACS 2019 spring national meeting\&Exposition, Mar 31-Apr 04, 2019, Orlando, FL.

Ruipeng Lei, David Butcher, Sophie Bernad, Valerie Derrien, Jaroslava Miksovska. Mutation of Residues in CD Loop and Distal Pocket Impact Protein Stability of Human Neuroglobin. 2019 Annual BSI Research Symposium, April 12, 2019, Miami, FL.

Ruipeng Lei, Maria Jose Santiago Estevez, Pierre Sebban, Sophie Bernad, Valerie Derrien, Jaroslava Miksovska. Study the Accessibility of the Heme Cavity In Hexacoordinate Globins By Using Fluorescent Heme Analog. 95 ${ }^{\text {th }}$ Florida Annual Meeting and Exposition (FAME) conference, May 9-11, 2019, Palm Harbor, FL.

Ruipeng Lei, David Butcher, Sophie Bernad, Valerie Derrien, Jaroslava Miksovska. Mutation of Residues in CD Loop and Distal Pocket Impact Protein Stability of Human Neuroglobin. FIUMASS event, July 19th, 2019, Miami, FL.

Ruipeng Lei, Maria Jose Santiago Estevez, Manuel Picon, Isa Sabir, Valerie Derrien, Sophie Bernad, Jaroslava Miksovska. Impact of the internal disulfide bond on the ligand migration in globin X. $64^{\text {th }}$ Biophysical society meeting, Feb 15-19, 2020, San Diego, CA. 